Christoph Anton Cordes

\title{
Gesamtrechtsnachfolge, \\ Datenschutzrecht und Vertragsgestaltung
}

Die Rechtsverhältnisse zwischen Provider und Nutzer im Nachlass 
Schriften zum Medien- und Informationsrecht

herausgegeben von

Prof. Dr. Boris P. Paal, M.Jur.

Band 54 
Christoph Anton Cordes

\section{Gesamtrechtsnachfolge, Datenschutzrecht und Vertragsgestaltung}

Die Rechtsverhältnisse zwischen Provider und Nutzer im Nachlass 
Gedruckt mit freundlicher Unterstützung des Vereins zur Förderung des Deutschen, Europäischen und Vergleichenden Wirtschaftsrechts e.V.

Die Deutsche Nationalbibliothek verzeichnet diese Publikation in der Deutschen Nationalbibliografie; detaillierte bibliografische Daten sind im Internet über http://dnb.d-nb.de abrufbar.

Zugl.: Freiburg, Univ., Diss., 2021

\section{Auflage 2021 \\ (c) Christoph Anton Cordes}

Publiziert von

Nomos Verlagsgesellschaft mbH \& Co. KG Waldseestraße 3-5 | 76530 Baden-Baden www.nomos.de

Gesamtherstellung:

Nomos Verlagsgesellschaft mbH \& Co. KG Waldseestraße 3-5 | 76530 Baden-Baden

ISBN (Print): 978-3-8487-8153-9

ISBN (ePDF): 978-3-7489-2582-8

DOI: https://doi.org/10.5771/9783748925828

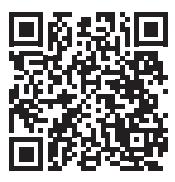

\section{(9) $(9 \Theta$}

Dieses Werk ist lizenziert unter einer Creative Commons Namensnennung - Nicht kommerziell - Keine Bearbeitungen 4.0 International Lizenz. 
Meinen Eltern 


\section{Vorwort}

Die vorliegende Arbeit wurde von der Juristischen Fakultät der AlbertLudwigs-Universität Freiburg im Wintersemester 2020/21 als Dissertation angenommen. Literatur und Rechtsprechung konnten bis Ende Oktober 2020 berücksichtigt werden.

Mein erster Dank gilt meinem verehrten Doktorvater, Prof. Dr. Boris $P$. Paal, M.Jur. (Oxford), für die in jeder Hinsicht hervorragende Betreuung und für die Förderung, die er mir zu jedem Zeitpunkt der Bearbeitung zukommen ließ. Ohne seine konstruktiven Anregungen und seine außerordentlich engagierte Unterstützung hätte die Arbeit nicht in dieser Form entstehen können. Ferner danke ich Prof. Dr. Sonja Meier, LL.M. (London) für die intensive Auseinandersetzung mit meiner Arbeit und für die zügige Erstellung des Zweitgutachtens.

Ich danke dem Studienförderwerk Klaus Murmann der Stiftung der Deutschen Wirtschaft für die mir zuteilgewordene Promotionsförderung sowie dem Verein zur Förderung des Deutschen, Europäischen und Vergleichenden Wirtschaftsrechts e.V. für die Gewährung eines großzügigen Druckkostenzuschusses.

Dr. Anne-Sophie Landwers hat ihre wissenschaftliche Erfahrung freigiebig mit mir geteilt und mich dadurch immer wieder ermutigt - vielen Dank dafür. In den letzten Jahren konnte ich mich zu wahrlich jedem Zeitpunkt und in jeder Hinsicht auf den unschätzbaren Rückhalt von Franziska Grethe, Laura Klein und Johannes Ostermeier verlassen. Dafür danke ich ihnen dreien von ganzem Herzen.

Schließlich bin ich meinen Schwestern, Clara und Lisa Cordes, und meinen Eltern, Maria und Albrecht Cordes, zu allergrößtem Dank verpflichtet. Keine Worte können ausdrücken, wie sehr ich all das schätze, was sie seit so vielen Jahren für mich tun. Ihnen und meinem Patensohn, Vincent, ist diese Arbeit gewidmet.

Berlin, im Februar 2021

Christoph A. Cordes 


\section{Inhaltsverzeichnis}

$\begin{array}{ll}\text { Abkürzungsverzeichnis } & 15\end{array}$

Kapitel 1 - Einleitung 23

A. Gegenstand der Untersuchung 25

B. Thematische Ein- und Ausgrenzungen 27

C. Gang der Untersuchung 28

D.Ziel der Untersuchung 30

Kapitel 2 - Bestimmung des Erbrechtsobjekts 31

A. Rechtsverhältnisse zwischen Provider und Nutzer 32

I. Vertragstypologie der Nutzungsverträge 33

1. Praktische Relevanz der Typenbestimmung 33

2. Vielgestaltigkeit der Hauptleistungspflichten 34

3. Typengemischte Verträge 35

4. Mehrseitiges Finanzierungsmodell der Provider 37

a) Dienste gegen Daten 38

b) Unentgeltlichkeit des Nutzungsvertrags 39

5. Zwischenergebnis zur Vertragstypologie von Nutzungsverträgen $\quad 41$

II. Urheber- und Leistungsschutzrechte des Erblassers 42

III. Zwischenergebnis zu A.

B. Daten als solche - (k)ein Recht an Daten 44

I. Einführung in die Debatte $\quad 45$

II. Konstruktionsvorschläge nach geltendem Recht 48

1. Sachenrecht 48

2. Deliktsrechtlicher Schutz von Daten 50

a) Recht am Datenbestand als „Sonstiges Recht“ iSv. $\$ 823$ Abs. 1 BGB 51

b) Deliktsrechtlicher Schutz von Daten gem. $₫ 823$ Abs. 2 BGB 52

c) Rückschlüsse aus dem Deliktsrecht auf den digitalen Nachlass 53

d) Zwischenergebnis $\quad 55$ 
3. Urheberrecht

a) Urheberrechtsschutz an Sammel- und Datenbankwerken gem. \$4 UrhG

b) Leistungsschutz des Datenbankherstellers gem. \S $87 \mathrm{a}$ ff. UrhG

c) Bezug zum digitalen Nachlass: Rechtsinhaberschaft und Reichweite des Rechts

4. Datenschutzrecht

5. (Lauterkeitsrechtlicher) Leistungs- und Geheimnisschutz

a) Lauterkeitsrechtlicher Leistungsschutz, $\mathbb{\int} 3$ Abs. 1, 4 Nr. 3 UWG

b) Geheimnisschutz, $\mathbb{S} \mathbb{S} 1 \mathrm{ff}$. GeschGehG

c) Lauterkeitsrecht und digitaler Nachlass

6. Ergebnis: Kein Recht an Daten de lege lata 66

III. Ausblick: Rechtspolitische Diskussion 66

IV. Rückschlüsse aus der Diskussion für den digitalen Nachlass $\quad 68$

C. Zwischenergebnis zu Kapitel 2

Kapitel 3 - Die Rechtsverhältnisse zwischen Provider und Nutzer im Nachlass

A. Vorüberlegung: Anwendbares Recht / IPR 70

I. Ausgangspunkt: Internationales Erbrecht 71

II. Vertragsstatut bei Nutzungsverträgen als Erbrechtsobjekt $\quad 73$

1. Nutzungsverträge als Verbraucherverträge i.S.v. Art. 6 Rom I-VO

a) Tatbestand des Art. 6 Abs. 1 Rom I-VO 74

aa) Der Nutzer als Verbraucher $\quad 74$

bb) Ausüben bzw. Ausrichten der Tätigkeit durch den Anbieter

b) Rechtsfolge: Qualifikation abhängig von Rechtswahl 78

c) Zwischenergebnis $\quad 80$

2. Nutzungsverträge ohne Verbraucherbeteiligung 80

a) Allgemeine Vertragskollisionsnorm, Art. 4 Rom I-VO 80

b) Rechtswahlvereinbarung zwischen Unternehmern, Art. 3 Rom I-VO

c) Zwischenergebnis $\quad 83$

III. Sonstige Erbrechtsobjekte und ihre Anknüpfung 83

IV. Zusammenfassung 
I. Einführung: Vertragliche Rechtsverhältnisse als Erbrechtsobjekte

II. Höchstpersönlichkeit als die Grenze der Vererbbarkeit

1. Höchstpersönlichkeit im Gefüge zwischen Provider, Erblasser und Kommunikationspartnern des Erblassers

a) Der Rechtsgedanke des $₫ 399$ Fall 1 BGB im Erbrecht allgemein

b) Der Rechtsgedanke des $₫ 399$ Fall 1 BGB im digitalen Nachlass

aa) Beurteilung der Pflichten des Providers 91

bb) Beurteilung der Pflichten des Nutzers 93

cc) Zusammenfassung

c) Vertragsimmanenter Schutz der

Kommunikationspartner

aa) Konto- oder personenbezogene Pflicht des Providers

bb) Erwartungen des verständigen Nutzers

cc) Keine Verfügungsbefugnis nach Versenden einer Nachricht

dd) Zwischenergebnis zu c)

d) Reichweite des ererbten Rechts am Nutzungskonto $\quad 100$

aa) Die Rechtsprechung zum Girokonto im digitalen Nachlass

bb) Keine Vergleichbarkeit von Girokonto und online Accounts

cc) Zwischenergebnis zu d)

e) Zwischenergebnis zu 1.

2. Höchstpersönlichkeit im Verhältnis zwischen dem Erblasser, seinen nächsten Angehörigen und den Erben

a) Differenzierungstheorie und „Infektion“ 108

b) Einheitlichkeit des Nachlasses 109

aa) Praktikabilitätserwägungen 110

bb) Die Wertung der $\$ \$ 2047$ Abs. 2, 2373 BGB 111

cc) Funktion und Reichweite der Wahrnehmungsberechtigung

dd) Zwischenergebnis zu b)

c) Kein Ausschluss der schlichten Kenntnisnahme durch die Erben

d) Zwischenergebnis zu 2. 
3. Zwischenergebnis zu II.

III. Beschränkung des übergegangenen Rechts

1. Das Datenschutzrecht der EU-DatenschutzGrundverordnung

a) Anwendungsbereich der DS-GVO

aa) Kein postmortaler Datenschutz des Erblassers

120

bb) Kommunikationspartner des Erblassers als „betroffene Person“"

cc) Ausschluss des Anwendungsbereichs gem. Art. 2

Abs. 2 DS-GVO

dd) Zwischenergebnis zu a)

b) Erlaubnistatbestände des Art. 6 Abs. 1 DS-GVO

124

aa) Einwilligung, Art. 6 Abs. 1 S. 1 lit. a) DS-GVO

bb) Erfüllung eines Vertrags, Art. 6 Abs. 1 S. 1 lit. b) Var. 1 DS-GVO

(1) Die betroffene Person als Vertragspartei

(2) Erforderlichkeit i.S.v. Art. 6 Abs. 1 S. 1 lit. b) Var. 1 DS-GVO

(3) Zwischenergebnis zu bb)

cc) Wahrung berechtigter Interessen, Art. 6 Abs. 1

S. 1 lit. f) DS-GVO

(1) Berechtigte Interessen des Verantwortlichen bzw. Dritter

(2) Erforderlichkeit der Datenverarbeitung

(3) Kein Überwiegen der Interessen der betroffenen Person

(4) Kinder im Rahmen von Art. 6 Abs. 1 S. 1 lit. f) DS-GVO

(5) Widerspruchsrecht des Betroffenen, Art. 21 Abs. 1 S. 1 DS-GVO

(6) Zwischenergebnis zu cc)

c) Verarbeitung „sensibler“ Daten im Sinne von Art. 9 DS-GVO

aa) Das Verarbeitungsverbot aus Art. 9 Abs. 1 DSGVO

bb) Die Ausnahmetatbestände aus Art. 9 Abs. 2 DSGVO

cc) Zwischenergebnis zu c)

d) Zwischenergebnis zu 1. 
2. Fernmeldegeheimnis gem. $\$ 88$ TKG 148

a) Verpflichtung des Providers durch $₫ 88$ TKG 149

aa) Verpflichtung als Diensteanbieter im Sinne des TKG

bb) Verpflichtung als Diensteanbieter im Sinne des TMG

cc) Zwischenergebnis zu a) 152

b) Tatbestand von $₫ 88$ Abs. 3 TKG 152

aa) Herrschende Ansicht: Kein Verstoß gegen $₫ 88$ Abs. 3 S. 1 TKG 153

bb) Andere Ansicht: Verstoß gegen $₫ 88$ Abs. 3 S. 1 TKG 156

cc) Rechtsdogmatische oder rechtspolitische Lösung 157

c) Fazit zu 2. 158

3. Zwischenergebnis zu III. 158

IV. Gestaltungsspielraum der Vertragsparteien $\quad 159$

1. Regelungen im Wege der Individualabrede $\quad 160$

2. Regelung im Wege Allgemeiner Geschäftsbedingungen 162

a) Einbeziehungskontrolle $\quad 162$

b) Inhaltskontrolle 164

aa) (Faktischer) Ausschluss der Vererbbarkeit $\quad 166$

(1) $\$ 307$ Abs. 2 Nr. 1 BGB 168

(2) $\$ 307$ Abs. 2 Nr. 2 BGB 170

(3) Vorgaben für eine zulässige Klausel zur (Un-)Vererbbarkeit 172

(4) Frist-Variante 174

(5) Zwischenergebnis zu aa) 175

bb) Bestimmung zur Accountberechtigung 176

(1) Zivilrechtliche Beurteilung 176

(2) Datenschutzrechtliche Bewertung 178

(3) Zwischenergebnis zu bb) 180

cc) Legitimationsklauseln / Erbfolgenachweis $\quad 180$

(1) Nachweis der Erbenstellung 181

(2) Nachweis der Nachlasszugehörigkeit 183

dd) Zwischenergebnis zu b) $\quad 184$

3. Zwischenergebnis zu IV. 184

V. Zwischenergebnis zu B. 185 
C. Urheberrechtlich geschützte Accountinhalte als Erbrechtsobjekt $\quad 186$

I. Die Urheberrechte des Nutzers und ihre Vererbbarkeit $\quad 188$

1. Urheber- und Leistungsschutzrechte des Nutzers 189

a) Unterscheidung der Kontoinhalte nach Werkarten 189

b) Der Nutzer als Schöpfer im Sinne von $\$ 7$ UrhG 191

c) Zwischenergebnis zu 1.

2. Vererbung des Urheberrechts, $\$ 28$ Abs. 1 UrhG 192

3. Zwischenergebnis zu I. 194

II. Lizenz des Providers an Urheberrechten des Nutzers 194

1. Wirksame Vereinbarung über Nutzungsrechte 196

a) Übertragungszweckgedanke des $\$ 31$ Abs. 5 UrhG 196

b) AGB-Kontrolle, $\$ \$ 305 \mathrm{ff}$. BGB 198

aa) Leitbildverstoß, $\$ 307$ Abs. 2 Nr. 1 BGB 199

bb) Transparenzgebot, $\mathbb{} 307$ Abs. 1 S. 2 BGB 201

cc) Vorgaben für eine zulässige Klausel zur

Rechtseinräumung 203

c) Zwischenergebnis zu 1.

2. Bindung der Rechtsnachfolger an die Lizenzeinräumung 205

3. Zwischenergebnis zu II. 207

III. Berechtigung an den Accountinhalten kraft Urheberrechts 207

1. Recht des Urheberrechtsinhabers auf Löschung der Accountinhalte 208

2. Recht des Urheberrechtsinhabers auf Zugang zum Account

3. Zwischenergebnis zu III. 210

IV. Zwischenergebnis zu C. 210

D. Schlussbetrachtungen zu Kapitel $3 \quad 211$

Kapitel 4 - Fazit und Ausblick 213

I. Zusammenfassung in Thesen 214

$\begin{array}{ll}\text { II. Ausblick } & 217\end{array}$

$\begin{array}{ll}\text { Literaturverzeichnis } & 219\end{array}$ 


\section{Abkürzungsverzeichnis}

a.a.O. am angegebenen Ort

a.F. alte Fassung

a.A. andere(r) Ansicht

ABl. Amtsblatt der Europäischen Union

Abs. Absatz/ Absätze

AcP Archiv für die civilistische Praxis

a.E. am Ende

AEUV Vertrag über die Arbeitsweise der Europäische Union

AfP Archiv für Presserecht

AGB Allgemeine Geschäftsbedingungen

Anm. Anmerkung

AnwBl Online Anwaltsblatt Online

APR Allgemeines Persönlichkeitsrecht

Art. Artikel

B2C Business-to-Consumer

BDSG Bundesdatenschutzgesetz

BeckOK Beck'scher Online-Kommentar

BeckRS Beck-Online Rechtsprechung

Begr. Begründer

BGB Bürgerliches Gesetzbuch

BGH Bundesgerichtshof

BGHZ Entscheidungssammlung des Bundesgerichtshofs in $\mathrm{Zi}$ vilsachen

BKartA Bundeskartellamt

Brüssel Ia-VO Brüssel Ia-Verordnung, VO (EU) Nr. 1215/2012

bspw. beispielsweise

BT-Drs. Bundestagsdrucksache 
BVerfG Bundesverfassungsgericht

BVerfGE Entscheidungssammlung des Bundesverfassungsgerichts

bzw.

beziehungsweise

C2C Consumer-to-Consumer

CR Computer und Recht

DAV Deutscher Anwaltverein

ders. derselbe

d.h. das heißt

dies. $\quad$ dieselbe $(\mathrm{n})$

DJT Deutscher Juristentag

DNotZ Deutsche Notar-Zeitschrift

DS-GVO Datenschutz-Grundverordnung, VO (EU) Nr. 2016/679

DSRI Deutsche Stiftung für Recht und Informatik

DS-RL Datenschutzrichtlinie, RL (EG) Nr. 95/46

DuD Datenschutz und Datensicherheit

ebd. ebenda

EGBGB Einführungsgesetz zum Bürgerlichen Gesetzbuche

Einl. Einleitung

EKEK-RL Europäischer Kodex für die elektronische Kommunikation, RL (EU) Nr. 2018/1972

EMRK Europäische Menschenrechtskonvention

ePrivacy-RL Datenschutzrichtlinie für elektronische Kommunikation, RL (EG) Nr. 2002/58

ePrivacy-VO mögliche Nachfolgeregelung der ePrivacy- $R L$

ErbR Zeitschrift für die gesamte erbrechtliche Praxis

etc. et cetera

EU Europäische Union

EuErbVO Erbrechtverordnung, VO (EU) Nr. 650/2012

EuGH Europäischer Gerichtshof

EUV Vertrag über die Europäische Union 
EWiR Entscheidungen zum Wirtschaftsrecht

EWS Europäisches Wirtschafts- und Steuerrecht

f., ff. folgende

FamRZ Zeitschrift für das gesamte Familienrecht

FAQ Frequently Asked Questions

FD-ErbR Fachdienst Erbrecht

FD-ZVR Fachdienst Zivilverfahrensrecht

Fn. Fußnote

FS Festschrift

GBO Grundbuchordnung

gem. gemäß

GeschGehG Gesetz zum Schutz von Geschäftsgeheimnissen

GeschGehG-RL Geschäftsgeheimnisrichtlinie, RL (EU) Nr. 2016/943

GG Grundgesetz

ggf. gegebenenfalls

GRCh EU-Grundrechtecharta

GRUR Gewerblicher Rechtsschutz und Urheberrecht

GRUR Int. GRUR International: Journal of European and International IP Law

GRUR-Prax GRUR - Praxis im Immaterialgüter- und Wettbewerbsrecht

GWB Gesetz gegen Wettbewerbsbeschränkungen

GWR Gesellschafts- und Wirtschaftsrecht

HGB Handelsgesetzbuch

HK Handkommentar

h.M. herrschende Meinung

Hrsg. Herausgeber

HS Halbsatz

i.E. im Ergebnis 
i.e. id est

IM-Dienst Instant Messaging-Dienst

Inc. Incorporated

IPR Internationales Privatrecht

IPRB IP-Rechtsberater

i.S.d. im Sinne des

i.S.v. im Sinne von

IT Informationstechnologie

ITRB IT-Rechtsberater

IZVR Internationalen Zivilverfahrensrecht

jew. jeweils

jM juris - Die Monatszeitschrift

JuS Juristische Schulung

JZ Juristenzeitung

K\&R Kommunikation und Recht

Kap. Kapitel

KG Kammergericht

Kommission Europäische Kommission

krit. kritisch

KUG Kunsturhebergesetz

lit. littera/ Buchstabe

Ls. Leitsatz

m.w.N. mit weiteren Nachweisen

mind. mindestens

Mio. Million(en)

MittBayNot Mitteilungen des Bayerischen Notarvereins

MMR Zeitschrift für IT-Recht und Recht der Digitalisierung

Mrd. Milliarde(n) 
MüKo

NJOZ

NJW

NJW-RR

NK

NotBZ

Nr.

NRW

NVwZ

NWB

NWB-EV

NZFam

o.

o.g.

OTT-Dienst

OVG

PatG

PinG

PM

RDV

RL

Rn.

RNotZ

Rom I-VO

Rom II-VO

Rspr.

RStV
Münchener Kommentar

Neue Juristische Online-Zeitschrift

Neue Juristische Wochenschrift

NJW Rechtsprechung-Report Zivilrecht

Nomos Kommentar

Zeitschrift für die notarielle Beratungs- und Beurkundungspraxis

Nummer

Nordrhein-Westfalen

Neue Zeitschrift für Verwaltungsrecht

Neue Wirtschaftsbriefe - Steuer- und Wirtschaftsrecht

Neue Wirtschaftsbriefe - Erben und Vermögen

Neue Zeitschrift für Familienrecht

oben

oben genannte(s)

Over-the-top Dienst

Oberverwaltungsgericht

Patentgesetz

Privacy in Germany

Pressemitteilung

Recht der Datenverarbeitung

Richtlinie

Randnummer/ Randnummern

Rheinische Notar-Zeitschrift

Rom I-Verordnung, VO (EG) Nr. 593/2008

Rom II-Verordnung, VO (EG) Nr. 864/2007

Rechtsprechung

Rundfunkstaatsvertrag 
S.

sog.

st.

str.

StGB

TK

TKG

TMG

u.a.

UAbs.

UN

UPR

UrhG

UWG

v.

VR-RL

VG

vgl.

$\mathrm{VO}$

VuR

WM

WRP

WuW

z.B.

ZD

ZErb

ZEuP
Satz/Seite(n)

sogenannt/ sogenannte/ sogenanntes

ständige

strittig

Strafgesetzbuch

Telekommunikation

Telekommunikationsgesetz

Telemediengesetz

und andere/ unter anderem

Unterabsatz

United Nations

Urheberpersönlichkeitsrecht

Urheberrechtsgesetz

Gesetz gegen den unlauteren Wettbewerb

von/vom

Verbraucherrechte-Richtlinie, RL (EU) Nr. 2011/83

Verwaltungsgericht

vergleiche

Verordnung

Verbraucher und Recht - Zeitschrift für Wirtschafts- und Verbraucherrecht

Wertpapiermitteilungen

Wettbewerb in Recht und Praxis

Wirtschaft und Wettbewerb

zum Beispiel

Zeitschrift für Datenschutz

Zeitschrift für die Steuer- und Erbrechtspraxis

Zeitschrift für Europäisches Privatrecht 
ZEV

ZfPW

ZHR

Ziff.

ZKG

$\mathrm{ZPO}$

ZRP

ZUM

ZUM-RD
Zeitschrift für Erbrecht und Vermögensnachfolge

Zeitschrift für die gesamte Privatrechtswissenschaft Zeitschrift für das gesamte Handelsrecht und Wirtschaftsrecht

Ziffer

Zahlungskontengesetz

Zivilprozessordnung

Zeitschrift für Rechtspolitik

Zeitschrift für Urheber- und Medienrecht

ZUM-Rechtsprechungsdienst 


\section{Kapitel 1 - Einleitung}

Im Zuge der Digitalisierung stellen sich zahlreiche Fragen des Privatrechts in neuem Lichte. Das Recht und seine Eignung zur interessengerechten Konfliktlösung werden durch die ausgreifende Umwälzung aller Wirtschafts- und Lebensbereiche im Zusammenhang mit der Digitalisierung massiv herausgefordert. ${ }^{1}$ Ein Teilbereich dieses großen Feldes wird in jüngerer Zeit an der Schnittstelle von Digitalisierung und Erbrecht unter dem Stichwort „Digitaler Nachlass“ diskutiert. Unter „Nachlass“ versteht das BGB (synonym mit Erbschaft) ${ }^{2}$ die Gesamtheit der auf den Erben übergegangenen Rechtsverhältnisse des Erblassers unter Einschluss der Verbindlichkeiten. "Digital“ wird gemeinhin als der Gegenbegriff zu analog verwendet und beschreibt maschinenlesbar codierte Informationen. ${ }^{4}$ Der „digitale Nachlass" bildet als begriffliche Neuschöpfung die Synthese dieser Bestandteile, ohne aber in der Debatte einheitlich verwendet zu werden. In der Literatur wurden mehrere Definitionsversuche mittels umfangreicher Beispielkataloge unternommen. ${ }^{5}$ Dem liegt ein Verständnis des digitalen Nachlasses als Sammelbegriff für eine bestimmte Gruppe von Erbrechtsobjekten zugrunde. Dazu sollen alle Rechtsverhältnisse des Erblassers gehören, welche informationstechnische Systeme betreffen einschließ-

1 Vgl. nur Schweitzer, ZEuP 2019, 1, 1 f. m.w.N. („Die Digitalisierung fordert das Privatrecht in seinen Grundfesten heraus. Durchgängige Grundlage der strukturellen Veränderungen ist die neue Bedeutung von Daten.").

2 Leipold in: MüKo BGB, $\mathbb{1} 1922$ Rn. 18 m.w.N.; ders., Erbrecht, Rn. 33 a.E.; Weidlich in: Palandt, $\mathbb{\$} 1922$ BGB Rn. 7.

3 Weidlich in: Palandt, $\mathbb{1} 1922$ BGB Rn. 7; Leipold in: MüKo BGB, $\mathbb{1} 1922$ Rn. 16 f.

4 Zech, Information als Schutzgegenstand, S. 32 f.; Alexander, K\&R 2016, 301, 302; Bock, AcP (217) 2017, 370, 372.

5 Vgl. Kroiß in: NK-BGB, $\mathbb{S} 1922$ Rn. 11; Kunz in: Staudinger, $\mathbb{S} 1922$ BGB Rn. 595. Etwa nach Ludyga, jM 2016, 442, 443 gehören zum digitalen Nachlass u.a. sämtliche Daten, die der Erblasser auf analogen Speichermedien oder in der Cloud gespeichert hat einschließlich der entsprechenden Soft- und Hardware, die Vertragsbeziehungen des Erblassers zu Anbietern von Kommunikations- und Informationsdiensten einschließlich der erforderlichen Nutzerkonten, die bei der Nutzung dieser Dienste entstehenden Kommunikationsdaten wie E-Mails und Chatverläufe, Rechte am Webauftritt einschließlich der Domain sowie Urheberrechte des Erblassers z.B. an Blogeinträgen, digitalen Fotos und Videos. 
lich des gesamten elektronischen Datenbestandes des Erblassers. ${ }^{6}$ Davon ausgehend ließe sich unter dem Begriff die Gesamtheit des digitalen Vermögens des Erblassers verstehen. ${ }^{7}$

Wie die Arbeit zeigen wird, ist es indes gerade ein weitgehend ungelöstes Problem, ob und wie Daten einem bestimmten Rechtsinhaber zugeordnet werden können. ${ }^{8}$ Daher und mit Blick auf Abgrenzungsschwierigkeiten zwischen analogen und digitalen Nachlassgegenständen - man denke an ein körperliches Speichermedium wie etwa eine Festplatte unter Einschluss der darauf gespeicherten Daten - erscheint ein Begriffsverständnis vorzugswürdig, welches nicht von einer klar abgrenzbaren digitalen Vermögensmasse ausgeht. Stattdessen kann der Wert der Bezeichnung in ihrem Schlagwortcharakter gesehen werden: Danach wird unter dem Stichwort „digitaler Nachlass“ im weitesten Sinne nach den juristischen Konsequenzen für das Erbrecht in einer zunehmend digitalisierten Welt gefragt. ${ }^{9}$ Sorge ${ }^{10}$ spricht insofern von einem „empirischen Realtypus“ als Untersuchungsgegenstand.

Angesichts der technischen und demographischen Entwicklung sind die komplexen rechtlichen Fragen, denen sich diese Arbeit widmet, zugleich von wachsender gesellschaftlicher und wirtschaftlicher Relevanz. ${ }^{11}$ Die Digitalisierung hat seit weit über zwei Jahrzehnten die breite Öffentlichkeit erreicht und erfasst sukzessive praktisch sämtliche öffentlichen und privaten Lebensbereiche. Die dabei entstehenden Daten werden von und für eine wachsende Anzahl von Nutzern erstellt. Die Zahl der Nutzer, die in einem nicht nur vernachlässigbaren Umfang digitale Spuren hinterlassen, nimmt in allen Alterskohorten zu. In der Konsequenz werden Erbfälle, die

6 Deusch, ZEV 2014, 2, 2 f.; Bock, AcP (217) 2017, 370, 372.

7 So Bräutigam in: DAV-Stellungnahme Nr.34/2013, S. 93; Müller-Christmann in: BeckOK BGB, $\mathbb{1} 1922$ Rn. 99; in diese Richtung, ebenfalls mit umfangreichen Beispielkatalog, auch die Studie des Fraunhofer Instituts für Sichere Informationstechnologie, S. 29 f., abrufbar unter https://www.sit.fraunhofer.de/de/digitalernac hlass/ [17.11.2020].

8 Vgl. dazu S. $44 \mathrm{ff}$.

9 Budzikiewicz, AcP (218) 2018, 558, 560; sogar die Vorsorge und Abwicklung des Nachlasses einschließend Herzog/Pruns, Der digitale Nachlass in der Vorsorgeund Erbrechtspraxis, S. 7; vgl. auch Herzog, AnwBl Online 2018, 472, 472; Gloser, MittBayNot 2016, 101, 108.

10 Sorge, MMR 2018, 372, $372 \mathrm{f}$.

11 Herzog/Pruns, Der digitale Nachlass in der Vorsorge- und Erbrechtspraxis, S. 2; Kutscher, Der digitale Nachlass, S. $15 \mathrm{f}$. 
(auch) einen digitalen Nachlass zur Folge haben, perspektivisch weiter zunehmen. ${ }^{12}$

\section{A. Gegenstand der Untersuchung}

Untersuchungsgegenstand dieser Arbeit ist das Schicksal der Rechtsverhältnisse zwischen einem Anbieter von elektronischen Informations- und Kommunikationsdiensten und einem Nutzer in dem Moment, in dem der Nutzer verstirbt. ${ }^{13}$ Als Erbrechtsobjekte kommen damit zum einen der Nutzungsvertrag zwischen Erblasser und Provider ${ }^{14}$ und zum anderen etwaige Immaterialgüterrechte des Erblassers an den Inhalten seines Accounts in Betracht. Daraus ergibt sich als Untersuchungsgegenstand eine multipolare Rechtsbeziehung, die für den digitalen Nachlass typisch ist und welche eine angemessene Würdigung der Interessen aller Beteiligten erfordert. ${ }^{15}$ Denn neben dem Nutzer, der zum Erblasser wird, und dem Provider selbst sind dabei insbesondere die Erben und - sofern insoweit keine Personenidentität besteht - die nächsten Angehörigen des Erblassers zu berücksichtigen. Wenn und soweit die Erben nach dem Erbfall Zugang zu den im Account gespeicherten Kommunikationsdaten des Erblassers erhalten, ${ }^{16}$ sind dadurch auch die Interessen der Kommunikationspartner des Erblassers betroffen. Schließlich sind mitunter sonstige Dritte, wie etwa die Follower des Erblassers, in den Blick zu nehmen.

12 Ebd. (Fn. 11); ferner Alexander, K\&R 2016, 301, 301; ähnlich die Antragsbegründung der FDP-Bundestagsfraktion in BT-Drs. 19/14044.

13 Obly in: Schricker/Loewenheim (Hrsg.), $\$ 28$ UrhG Rn. 2a beschreibt diese Rechtsverhältnisse anschaulich als „ein Bündel von Rechten“.

14 Zum Providerbegriff im Allgemeinen s. Redeker in: Hoeren/Sieber/Holznagel (Hrsg.), MMR-HdB, Teil 12 Rn. 1 ff. mit Verweis auf Kosmides, Providing-Verträge, S. $156 \mathrm{ff}$. Hier wird der Begriff zur Bezeichnung von Anbietern von Kommunikations- und Informationsdiensten verwendet. Zu reinen Internet-Access-Providern im Kontext des digitalen Nachlasses vgl. Bräutigam/Herzog/Mayen/Redeker/ Zuck in: DAV-Stellungnahme Nr. 34/2013, S. 6 und 9 (zu \$43c TKG-E).

15 Nach Budzikiewicz, AcP (218) 2018, 558, $561 \mathrm{f}$. ist das Interessengeflecht zwangsläufig der Ausgangspunkt für die weitere Konkretisierung des digitalen Nachlasses als Untersuchungsgegenstand insgesamt; in diese Richtung auch Thiesen, Daten in der Erbmasse, S. 2 ff. und Raude, RNotZ 2017, 17, 24.

16 Dasselbe gilt, wenn anstelle der Erben eine andere Vertrauensperson, die vom Erblasser dazu bestimmt wurde, Zugang zum Account erhalten soll, s. dazu S. $176 \mathrm{ff}$. 
Die Interessen der unterschiedlichen Beteiligten können mitunter zueinander in Konflikt geraten. Diese Aussicht lässt eine Vielzahl ebenso unterschiedlicher wie vielschichtiger Interessenskollisionen in einem „Knäuel von Rechtsverhältnissen " ${ }^{\text {"17 }}$ erwarten. Zur Auflösung dieser Kollisionen sind die hinter den jeweiligen Interessen stehenden Regelungsregime im Verlauf der Untersuchung zu benennen, zu gewichten und in einen schonenden Ausgleich zu bringen. Dabei ist das Erbrecht zwar der Ausgangspunkt der juristischen Argumentation. Für sich allein genommen bietet es aber keine umfassende und abschließende Lösung der Problemlagen. Vielmehr ist daneben zwingend das Schuldrecht, insbesondere unter Einschluss des Rechts der allgemeinen Geschäftsbedingungen, zu berücksichtigen, soweit die vertragliche Stellung des Erblassers im Nutzungsverhältnis mit dem Provider als Erbrechtsobjekt untersucht wird. Sofern als Erbrechtsobjekte die Immaterialgüterrechte des Erblassers an den Accountinhalten beleuchtet werden, finden vor allem das Urheberrecht und dort insbesondere die Spezialbestimmungen des UrhG zum Erb- und Urhebervertragsrecht Anwendung. Schließlich sind in jedem Fall das Datenschutzund das Telekommunikationsrecht von hervorgehobener Bedeutung, auf deren Schutz sich die Kommunikationspartner des Erblassers gegebenenfalls (gegenüber dem Provider) berufen können. Nicht zuletzt müssen die Auslegung und Anwendung des einfachen Rechts in Einklang mit den Vorgaben des Verfassungsrechts erfolgen. Dabei sind hier hauptsächlich die verfassungsrechtlichen Gewährleistungen von Eigentum und Erbrecht, Art. 14 Abs. 1 GG, das postmortale Persönlichkeitsrecht des Erblassers, Art. 1 Abs. 1 GG, sowie das allgemeine Persönlichkeitsrecht der Kommunikationspartner, Art. 2 Abs. 1 GG in Verbindung mit Art. 1 Abs. 1 GG, zu berücksichtigen. Eine abstrakte Untersuchung getrennt nach den jeweiligen Rechtsgebieten erscheint indessen wenig zielführend, da gerade das Zusammenwirken und die Kollisionen der Regime die Komplexität des digitalen Nachlasses ausmachen. Daher wird sich die Arbeit über exemplarisch ausgewählte Erbrechtsobjekte dem digitalen Nachlass weiter nähern, indem für die Nachlassgegenstände mit digitalen Bezügen jeweils die Frage nach dem „Ob“ und nach dem „Wie“ ihrer Vererbbarkeit gestellt wird.

In einer viel beachteten Entscheidung hat der Bundesgerichtshof mit Urteil vom 12. Juli $2018^{18}$ über die Vererbbarkeit eines Facebook-Nutzerkon-

17 Sorge, MMR 2018, 372.

18 BGH NJW 2018, 3178. 
tos entschieden. ${ }^{19}$ Danach soll der Nutzungsvertrag zwischen dem Kontoinhaber und dem Anbieter des sozialen Netzwerks im Erbfall grundsätzlich gem. $\$ 1922$ Abs. 1 BGB auf die Erben übergehen. Dem Recht der Erben auf Zugang zu dem Benutzerkonto samt den darin gespeicherten Inhalten stehe „weder das postmortale Persönlichkeitsrecht des Erblassers noch das Fernmeldegeheimnis oder das Datenschutzrecht entgegen. " ${ }^{20}$ Die Entscheidungsgründe sind im weiteren Verlauf der Arbeit einer näheren Untersuchung zu unterziehen. Insbesondere ist dabei Augenmerk auf die Frage zu legen, ob und inwieweit die Ausführungen des Gerichts über Facebook hinaus auf andere Informations- und Kommunikationsdienste übertragen werden können. ${ }^{21}$ Dabei ist der großen Vielfalt des Angebots unterschiedlicher Internetdienste, die von ihren Nutzern zu beruflichen (wie z.B. LinkedIn), privaten (wie z.B. Instagram) und/oder intimen (wie z.B. Parship) Zwecken verwendet werden, Rechnung zu tragen.

\section{B. Thematische Ein- und Ausgrenzungen}

Die Tatsache, dass mit der Digitalisierung und dem Erbrecht zwei Querschnittsthemen vorliegen, die mit nahezu jedem Aspekt des Lebens in Verbindung kommen können, zwingt zu thematischen Ein- und Ausgrenzungen. Die Bestimmung des Untersuchungsumfangs ist dabei anhand der Rechtsobjekte vorzunehmen, die in den Nachlass des Erblassers fallen können. Die nutzungsvertragliche Position des Erblassers gegenüber dem Provider und etwaige Immaterialgüterrechte, die an den Accountinhalten des Erblassers bestehen, bilden gemeinsam die Rechtsverhältnisse zwischen den Anbietern von Informations- und Kommunikationsdiensten und ihren Nutzern, die den Gegenstand der vorliegenden Arbeit bilden sollen. Beide Erbrechtsobjekte eint regelmäßig ein starker (urheber-)persönlich-

$19 \mathrm{Zu}$ der Facebook-Entscheidung vgl. etwa Martini/Kienle, JZ 2019, 235; Lieder/ Berneith, FamRZ 2018, 1486; Leipold in: MüKo BGB, $\mathbb{1} 1922$ Rn. 32 ff.; Alexander, notar 2018, 367; Ludyga, ZEV 2018, 592; Litzenburger, FD-ErbR 2018, 407688; Apel, ZD 2018, 486; Wüsthof, ErbR 2018, 579; Biermann, ErbR 2018, 577; Deusch, ZEV 2018, 687; Gloser, DNotZ 2018, 859; Hoeren, MMR 2018, 749; Härting/Dag, K\&R 2018, 638; Preuß, NJW 2018, 3146.

20 BGH NJW 2018, 3178, Ls.

21 LG Münster ErbR 2019, 455 bejaht die Vererbbarkeit eines iCloud-Accounts. Zur Begründung seines Versäumnisurteils verweist das Gericht auf BGH NJW 2018, 3178. Hierbei handelt es sich - soweit ersichtlich - um das bislang einzige andere Verfahren vor deutschen Gerichten zu der aufgeworfenen Frage. 
keitsrechtlicher Bezug zum Erblasser. Die Rechtsverhältnisse sind zum einen daraufhin zu untersuchen, ob sie vererbbar sind. Zum anderen ist zu beleuchten, ob und inwieweit die Rechtsnachfolger bei der Wahrnehmung der auf sie übergegangenen Rechtsposition Beschränkungen unterliegen.

Andere Rechtsobjekte hingegen, die zwar ebenfalls in den Nachlass fallen und einen digitalen Bezug aufweisen, ohne aber in vergleichbarer Weise persönlichkeitsrechtlich geprägt zu sein, sollen hingegen nicht Gegenstand der vorliegenden Untersuchung sein. Das schließt insbesondere das Erbrecht an sogenannten Kryptowährungen wie beispielsweise Bitcoin, ${ }^{22}$ an Domains ${ }^{23}$ sowie an körperlichen Speichermedien wie Festplatten ${ }^{24}$ aus. In urheberrechtlicher Hinsicht sind die absoluten Immaterialgüterrechte des Erblassers, die Gegenstand der Untersuchung sind, von seinen urheberrechtlichen Lizenzen an Werken Dritter, wie etwa im Rahmen einer ITunes-Mediathek, ${ }^{25} \mathrm{zu}$ unterscheiden. Letztere sollen hier ebenfalls nicht näher in ihrer Qualität als Erbrechtsobjekte untersucht werden.

\section{Gang der Untersuchung}

Um die Vererbbarkeit einer Rechtsposition zu beurteilen, ist zunächst eine nähere Bestimmung des Erbrechtsobjekts unerlässlich. Dieser Ausgangsfrage widmet sich - im Anschluss an dieses erste Kapitel - das Kapitel 2 der vorliegenden Untersuchung, das sich in zwei Teile gliedert. ${ }^{26} \mathrm{Im}$ ersten Teil sind die Rechtsverhältnisse zwischen dem Anbieter des Internetdienstes und dem Nutzer konkret zu benennen. Dafür ist insbesondere eine vertragstypologische Einordnung des Nutzungsvertrags unter besonderer Be-

22 Vgl. dazu Amend-Traut/Hergenröder, ZEV 2019, 113.

23 Vgl. dazu Viefhues in: Hoeren/Sieber/Holznagel (Hrsg.), MMR-HdB, Teil 6 Rn. 406 sowie bereits Hoeren, NJW 2005, 2113, 2115 f.

$24 \mathrm{Zu}$ der Frage, ob über die faktische Zugriffsmöglichkeit des nachgefolgten Sacheigentümers hinaus eine (vererbbare) Berechtigung an den gespeicherten Daten besteht, die dem Eigentum an dem Speichermedium folgt, s. bejahend etwa Budzikiewicz, AcP (218) 2018, 558, 567; Ludyga, jM 2016, 442, 443; Herzog, AnwBl Online 2018, 472, 475 f.; Kutscher, Der digitale Nachlass, S. 100; differenzierend Hoeren, NJW 2005, 2113, 2114; Martini, JZ 2012, 1145, $1147 \mathrm{ff} . ;$ Kunz in: Staudinger, $\$ 1922$ BGB Rn. $607 \mathrm{ff}$.

25 Vgl. dazu Lange/Holtwiesche, ZErb 2016, 125, 128 ff.; Herzog/Pruns, Der digitale Nachlass in der Vorsorge- und Erbrechtspraxis, S. $107 \mathrm{ff}$.; Studie des Fraunhofer Instituts für Sichere Informationstechnologie, S. 131 ff., abrufbar a.a.O. (Fn. 7).

$26 \mathrm{Zu}$ Kapitel 2 s. S. $31 \mathrm{ff}$. 
rücksichtigung des mehrseitigen Finanzierungsmodells der Provider vorzunehmen. Daneben sind die Urheber- und Leistungsschutzrechte nach Maßgabe das UrhG des Erblassers an in den Account eingebrachten Inhalten zu beleuchten. Im zweiten Teil ist die Debatte um ein (eigentumsähnliches) Recht an Daten mit der vorliegenden Thematik des digitalen Nachlasses zu verknüpfen. Dabei sind sowohl die Diskussion zum geltenden Recht als auch die rechtspolitische Debatte um mögliche Reformen danach zu befragen, ob und inwieweit ein etwaig bestehendes oder künftig zu erlassendes Recht an Daten einen Einfluss auf die Bestimmung und die rechtliche Zuordnung des Erbrechtsobjekts im digitalen Nachlass haben kann.

Auf Grundlage der nunmehr konkret bestimmten Erbrechtsobjekte dient das anschließende Kapitel 3 dazu, das Schicksal der Rechtsverhältnisse zwischen Provider und Nutzer im Erbfall zu untersuchen. Dieses Kapitel 3 ist in drei Teile gegliedert. ${ }^{27} \mathrm{Da}$ der digitale Nachlass typischerweise durch grenzüberschreitende Elemente geprägt ist, sind der Untersuchung in einem ersten Teil zunächst internationalprivatrechtliche Vorüberlegungen zum anwendbaren Recht voranzustellen. Daran schließt sich der zweite Teil an, in dem auf Grundlage des deutschen Rechts der Nutzungsvertrag als Erbrechtsobjekt untersucht wird. Zentral ist dabei die Frage, ob und inwieweit die nutzungsvertragliche Position des Erblassers vererbbar ist. Dafür sind insbesondere Aspekte des allgemeinen und postmortalen Persönlichkeitsrechts der beteiligten Akteure in den Blick zu nehmen. Sodann sind - den Übergang der Position auf die Erben unterstellt - etwaige Beschränkungen des übergegangenen Rechts zu prüfen. Diese können sich zum einen aus dem Datenschutzrecht der europäischen DatenschutzGrundverordnung ergeben sowie zum anderen aus dem Telekommunikationsrecht. Schließlich ist der (formularvertragliche) Gestaltungsspielraum der Parteien des Nutzungsvertrags hinsichtlich erbrechtlicher Aspekte der Vertragsgestaltung auszuloten. Im dritten Teil sind sodann die Urheberund Leistungsschutzrechte an den Accountinhalten als Erbrechtsobjekte zu untersuchen. Dabei soll insbesondere beleuchtet werden, in welchem Verhältnis die Inhaberschaft an den Immaterialgüterrechten zu der nutzungsvertraglichen Position steht.

Im abschließenden Kapitel 4 ist nach einer Zusammenfassung der gefundenen Ergebnisse in Thesen sodann ein Ausblick auf die weitere Entwicklung des digitalen Nachlasses zu wagen. ${ }^{28}$

$27 \mathrm{Zu}$ Kapitel 3 s. S. 70 ff.

28 Zu Kapitel 4 s. S. 213 ff. 


\section{Ziel der Untersuchung}

Die Erscheinungsformen daten- und internetbasierter Dienste sind vielfältig und differieren mitunter erheblich sowohl bezüglich ihrer Funktion für den Nutzer als auch in Hinblick auf ihre technische Ausgestaltung. Ziel der Arbeit ist es, unter Berücksichtigung dieser Unterschiede auszuloten, ob und inwieweit sich verallgemeinerbare Parameter zur Abwicklung des digitalen Nachlasses entwickeln lassen und in welchen Konstellationen das geltende Recht eine einzelfallspezifische Differenzierung erfordert. Es stellt sich die Frage, ob und unter welchen Voraussetzungen zwischen zwei Nutzerkonten des Erblassers unterschieden werden muss, wenn beispielsweise das eine Konto geschäftlichen ${ }^{29}$ und das andere Konto privaten oder gar intimen ${ }^{30}$ Zwecken dient. Bei der Beantwortung dieser Frage gilt es die Differenzierungspotenziale der jeweils zur Anwendung berufenen Regelungsregime zu benennen und fruchtbar zu machen, um auf diese Weise etwaig kollidierende Interessen der Beteiligten zu einem möglichst schonenden Ausgleich zu bringen. Vor diesem Hintergrund müssen auch die Entscheidungsgründe des BGH aus dessen Urteil zur Vererbbarkeit eines Facebook-Accounts ${ }^{31}$ auf ihre Verallgemeinerungsfähigkeit für andere Internetdienste hin kritisch überprüft werden. Ein weiteres Ziel dieser Arbeit ist es dabei, die (formularvertraglichen) Gestaltungsmöglichkeiten der Beteiligten - namentlich des Erblassers und der Provider - in den Blick zu rücken und deren Grenzen zu bestimmen.

29 Für ein anschauliches Beispiel siehe Podszun, GWR 2016, 37, 37 („twitternder Anwalt").

30 Etwa das Gutachten der Datenethikkommission vom 23.10.2019, S. 111, plädiert für eine Sonderbehandlung von „besonders persönlichkeits-sensitiv[en Nutzerkonten wie] etwa ein Online-Konto in einer Gruppe ,Anonymer Alkoholiker“", abrufbar unter https://www.bmi.bund.de/SharedDocs/downloads/DE/publikatio nen/themen/it-digitalpolitik/gutachten-datenethikkommission.pdf?_blob=publi cationFile $\& v=6[17.11 .2020]$.

31 BGH NJW 2018, 3178. 


\section{Kapitel 2 - Bestimmung des Erbrechtsobjekts}

Den digitalen Nachlass zum Untersuchungsgegenstand zu machen, heißt auch grundsätzlich über datenbasierte Vermögensgegenstände nachzudenken. Der „empirische Realtypus digitaler Nachlass“32 weist Berührungspunkte mit einer großen Bandbreite unterschiedlicher Rechtsobjekte auf. ${ }^{33}$ Ausgangspunkt der Frage nach dem „Ob“ und dem „Wie“ der Vererbbarkeit dieser Rechtsobjekte muss es sein, sie als konkrete Bestandteile des (digitalen) Nachlasses zu benennen und sie in ihrer rechtlichen Qualität zu bestimmen. Entsprechend dem Zuschnitt der Arbeit sollen hierzu die Rechtsverhältnisse zwischen dem Anbieter von Informations- und Kommunikationsdiensten und seinem jeweiligen Nutzer untersucht werden: De lege lata ist der zwischen diesen Parteien geschlossene Nutzungsvertrag der Hauptanknüpfungspunkt für die rechtliche Zuordnung der Nutzerkonten $^{34}$ des Erblassers. Daneben können Immaterialgüter-, insbesondere Urheberrechte, an den Accountinhalten bestehen. ${ }^{35}$ Der Nutzungsvertrag und die Urheberrechte des Erblassers bilden in ihrer Eigenschaft als Erbrechtsobjekte damit den zentralen Untersuchungsgenstand dieser Arbeit. Einführend ist daher ein näherer Blick auf diese Rechtspositionen zu werfen (dazu A.). Im Anschluss soll die verwandte Debatte zu möglichen originären Rechtspositionen an Daten dargestellt werden (dazu B.). Dem Nutzer mag eine affektive, umgangssprachliche Einteilung in „eigene“ Daten und andere Daten mitunter möglich sein. ${ }^{36}$ So ist es etwa denkbar, dass ein Arbeitnehmer die Inhalte und insbesondere die Nachrichten, die er auf

32 Sorge, MMR 2018, 372, $372 \mathrm{f}$.

33 Siehe hierzu bereits die Nachweise in Fn. 5, 6 und 7.

34 Zur rechtlichen Einordnung eines Accounts als solchem vgl. Kutscher, Der digitale Nachlass, S. 21 ff.; Seidler, Digitaler Nachlass, S. 69 ff. Nach hier vertretener Ansicht kommt ein Recht am Account als solchem allenfalls unter dem Gesichtspunkt eines Rechts an Daten in Betracht, s. zu diesem S. $44 \mathrm{ff}$.

$35 \mathrm{Zu}$ der Unterscheidung zwischen E-Mail und E-Mail-Account als erster Hoeren, NJW 2005, 2113, $2114 \mathrm{f}$; instruktiv dazu Bräutigam in: DAV-Stellungnahme Nr.34/2013, S. 17 f.; sich Hoeren anschließend Brisch/Müller-ter Jung, CR 2013, 446, 447; s. auch Herzog, NJW 2013, 3745, 3749; dies./Pruns, Der digitale Nachlass in der Vorsorge- und Erbrechtspraxis, S. $66 \mathrm{f}$.

36 Richter, Vorwort, in: Stiftung Datenschutz (Hrsg.), Dateneigentum und Datenhandel, S. 5; kritisch zu diesem Selbstverständnis der Nutzer - vor allem auch in Data-for-Access-Konstellationen - vgl. Amstutz, AcP 218 (2018), 439, 439 ff. 
seinem (auch) privat genutzten Dienstgerät abgespeichert hat, in private („eigene“) und dienstliche Daten unterteilen könnte. ${ }^{37}$ Allerdings ist es fraglich, ob und inwieweit diese affektive Beziehung einer Person zu „ihren“ Daten gegenwärtig rechtlich abgebildet wird. Sofern ein originäres, eigentumsähnliches Recht des Nutzers an Daten künftig anerkannt oder vom Gesetzgeber geschaffen wird, könnte sich daraus jedenfalls ein (zusätzliches) Erbrechtsobjekt oder sogar eine Vereinheitlichung der Erbrechtsobjekte im digitalen Nachlass ergeben.

\section{A. Rechtsverhältnisse zwischen Provider und Nutzer}

Dauerschuldverhältnisse sind von wesentlicher Bedeutung in der Digitalwirtschaft. Die immer größere Verfügbarkeit von (Online-)Speicherplatz zu immer geringeren Kosten hat zur Folge, dass die Kundenerfahrung des Nutzers an vielen Punkten nicht mehr durch körperliche Gegenstände geprägt ist. Stattdessen wurden die körperlichen Gegenstände durch digitale Versionen ersetzt. Man denke nur an Briefpost, die nunmehr als E-Mail oder Chatnachricht vorliegt, oder körperliche Musik-CDs oder FilmDVDs, die zunehmend durch Streaming-Angebote ersetzt werden. ${ }^{38}$ Während der Zugriff auf die körperlichen Gegenstände regelmäßig durch das an ihnen bestehende Eigentum bestimmt wird, sind es demgegenüber Nutzungsverträge, die in den meisten Fällen das Recht auf Zugang zu den digitalen Dateien regeln. Das gilt jedenfalls, wenn und soweit die Dateien auf Servern des Providers und nicht auf privaten Endgeräten des Nutzers gespeichert sind. Denn in diesem Fall kommt aus Sicht des Nutzers und seiner Rechtsnachfolger das Sacheigentum am physischen Speichermedium als Erbrechtsobjekt nicht in Betracht. Nutzungsverträge, in deren Rahmen beispielsweise die Mitgliedschaft in sozialen Netzwerken beziehungsweise der Zugang zur digitalen Infrastruktur des Plattformbetreibers vereinbart wird, erhalten dadurch eine Schlüsselfunktion. Im Folgenden sollen die Nutzungsverträge typologisch untersucht werden, wozu das mit

37 Im Falle eines unter Eigentumsvorbehalt erworbenen Endgerätes dürfte ein affektives Zuordnungsverhältnis zu (fast) allen abgespeicherten Daten bestehen, da der Nutzer ein solches Gerät von Anfang an als „sein eigenes“ ansieht und entsprechend unterschiedslos private Daten abspeichert.

$38 \mathrm{Zu}$ den Auswirkungen der Digitalisierung auf die Nutzung von urheberrechtlich geschützten Werken s. Sucker, Der digitale Werkgenuss im Urheberrecht, S. $69 \mathrm{ff}$. Zur Behandlung urheberrechtlicher Lizenzen des Erblassers an Werken Dritter im Kontext des digitalen Nachlasses vgl. bereits die Nachweise in Fn. 25. 
der Vertragstypenbestimmung in engem Zusammenhang stehende mehrseitige Finanzierungsmodell der Provider darzustellen ist (dazu I.). Im Anschluss sind die Urheber- und Leistungsschutzrechte des Erblassers an einzelnen Inhalten des Accounts sowohl als eigenständige Rechtsposition als auch in ihrem Verhältnis zum Nutzungsvertrag zu beleuchten (dazu II.).

\section{Vertragstypologie der Nutzungsverträge}

Um eine einzelfallgerechte Typologisierung der Nutzungsverträge vornehmen zu können, sind nach einführenden Bemerkungen zur praktischen Relevanz der Typenbestimmung (dazu 1.), die unterschiedlichen Hauptleistungspflichten des Providers (dazu 2.) und der Umgang mit typengemischten Verträgen (dazu 3.) zu untersuchen. Zur Bestimmung der Parteiinteressen und des Vertragszwecks ist sodann der Fokus auf das mehrseitige Finanzierungsmodell der Provider zu richten (dazu 4.).

\section{Praktische Relevanz der Typenbestimmung}

Die typologische Einordnung der Verträge zwischen den Diensteanbietern und den Nutzern scheint zunächst von nur untergeordneter praktischer Bedeutung zu sein, da die vertraglichen Rechte und Pflichten der Parteien in den Nutzungsbedingungen der Anbieter regelmäßig ohnehin detailliert ausformuliert werden. ${ }^{39}$ Allerdings darf nicht übersehen werden, dass dem dispositiven Recht eines Vertragstypus gem. $\$ 307$ Abs. 2 Nr. 1 BGB eine Leitbildfunktion bei der Inhaltskontrolle der AGB zukommt. ${ }^{40}$ Insofern gibt die Qualifizierung des Nutzungsvertrags als ein Vertragstyp des BGB den Rahmen der Gestaltungsmöglichkeiten für den Verwender der AGB vor. Darüber hinaus folgt aus der Einordnung, welche Vorschrift dann zur Anwendung kommt, wenn die AGB des Providers ein Nutzungsverhältnis doch einmal nicht ganz umfassend geregelt haben sollten. ${ }^{41}$ Für die internationalprivatrechtliche Frage nach dem anwendbaren Recht spielt aus-

$39 \mathrm{Kindl}$, Verträge über digitale Inhalte - Vertragsnatur und geschuldete Leistung, in: Kindl/Arroyo/Gsell (Hrsg.), Verträge über digitale Inhalte und digitale Dienstleistungen, S. 68.

40 Kindl a.a.O. (Fn. 39); Kutscher, Der digitale Nachlass, S. 45; Boehm, ZEuP 2016, $358,363$.

41 Boehm, ZEuP 2016, 358, 363; Stieper in: FS Helmut Köhler, S. 730 f. 
weislich des Art. 4 Abs. 1 Rom I-Verordnung die Vertragstypologie ebenfalls eine zentrale Rolle. ${ }^{42}$ Vor diesem Hintergrund kann der Typenbestimmung im Einzelfall durchaus eine entscheidende Funktion zukommen.

\section{Vielgestaltigkeit der Hauptleistungspflichten}

Die Bestimmung eines Vertragstyps erfolgt im Allgemeinen anhand des Vertragsinhalts und genauer anhand der Hauptleistungspflichten der Parteien. Im Bereich der Digitalwirtschaft wird es regelmäßig die Pflicht des Diensteanbieters sein, welche kennzeichnend für die Typenbestimmung ist. ${ }^{43}$ Der Anbieter stellt dem Nutzer im Rahmen eines nutzungsbeschränkten IT-Systems einen Account zur Verfügung. Je nach vorgesehenem Nutzungszweck dieses Accounts sind die Pflichten der Accountanbieter mitunter sehr unterschiedlich ausgestaltet. Dabei verpflichtet sich der Anbieter zur Eröffnung und Aufrechterhaltung der IT-Infrastruktur, die für die konkreten Dienste notwendig ist und regelmäßig online „Cloud“Speicherplatz für die Inhalte der Nutzer umfasst. Zu diesen Diensten kann eine Kommunikationsfunktion gehören, die mehreren Nutzern den Austausch untereinander ermöglicht. Dies kann durch Austausch von schriftlichen Nachrichten oder von Bildern, durch Audiodateien als Sprachnachrichten oder mittels internetbasierter (Video-)Telefonie erfolgen. In vielen Fällen wird die Kommunikationsfunktion um eine Möglichkeit der Vernetzung ergänzt, die es den Nutzern ermöglicht, Kontakte zu knüpfen und zu pflegen. In Betracht kommt weiter die Möglichkeit der Nutzer, Inhalte mit der Öffentlichkeit oder einem durch den Nutzer definierten Ausschnitt der Öffentlichkeit zu teilen. Spiegelbildlich ist es den Nutzern in diesen Fällen zumeist möglich, eine Auswahl darüber zu treffen, wessen Inhalte sie abonnieren wollen. Dadurch entsteht eine Plattform mit nut-

42 Sofern keine der in Art. 4 Abs. 1 Rom I-VO genannten Vertragsarten vorliegt, muss auf einen der Auffangtatbestände zurückgegriffen werden, vgl. Boehm, ZEuP 2016, 358, 363; Kutscher, Der digitale Nachlass, S. 85 ff. Die Begriffe und Vertragstypen der Rom I-VO sind freilich autonom auszulegen und unterscheiden sich ggf. von denen des deutschen Rechts. Ausführlich zum IPR s. S. $70 \mathrm{ff}$.

43 Bericht der Arbeitsgruppe „Digitaler Neustart“ der Konferenz der Justizministerinnen und Justizminister der Länder, Mai 2017, S. 197, abrufbar unter https://w ww.justiz.nrw.de/JM/schwerpunkte/digitaler_neustart/zt_bericht_arbeitsgruppe/ bericht_ag_dig_neustart.pdf [17.11.2020]; Bock, AcP (217) 2017, 370, 377; Budzikiewicz, AcP (218) 2018, 558, 569. 
zergenerierten Inhalten. ${ }^{44}$ Überdies existieren zahlreiche Mischformen, bei denen die Kommunikations-, Vernetzungs- und die Publikationsfunktion in unterschiedlichen Verhältnissen den Schwerpunkt des Netzwerks aus Sicht seiner Nutzer bestimmen. Bisweilen werden die Funktionen mit Blick auf ein übergeordnetes Thema ausgerichtet und ausgestaltet. Zu denken ist hier an berufliche Aspekte wie Karriereförderung und professionelle bzw. wissenschaftliche Vernetzung sowie an sonstige thematische Verknüpfung politischer, freizeitlicher, künstlerischer oder verschiedenster anderer Natur.

\section{Typengemischte Verträge}

Die Darstellung der Funktionen kann naturgemäß nur einen Ausschnitt der großen Vielfalt an Kommunikations- und Informationsdiensten abbilden. ${ }^{45}$ Die entstehende Bandbreite denkbarer Vertragstypen erfordert eine Einzelfallbetrachtung. Eine allgemeingültige Einordnung von ProviderVerträgen ist demgegenüber nicht zielführend. ${ }^{46}$ Das liegt auch an der ständigen Weiterentwicklung des konkreten Erscheinungsbilds der Internetdienste. ${ }^{47}$ Die konkrete Ausgestaltung der Dienste ist vor diesem Hintergrund immer nur eine „Momentaufnahme“, ${ }^{48}$ zu der laufend Funktionen hinzukommen, die einen weiteren Vertragstypus einschlägig erschei-

44 Geläufig ist auch die englische Bezeichnung User-generated content (UGC). Siehe zu dem Begriff Bauer, User Generated Content, S. 7 ff.; ders., User Generated Content, in: Große Ruse-Khan/Klass/v. Lewinski (Hrsg.), Nutzergenerierte Inhalte als Gegenstand des Privatrechts, S. 3 ff.

45 Anschaulich zur Vielfalt des Angebots formuliert Martini in: BeckOK Informations- und Medienrecht, $\mathbb{1} 1$ TMG Rn. 7: „Telemediendienste können in vielfältigen Erscheinungsformen des Wirtschaftslebens und zivilgesellschaftlichen Informationsaustausches auftreten [...]. Das Kaleidoskop ihrer Phänotypen entwickelt sich dynamisch. Der Versuch, einen abschließenden Katalog von Telemedien aufzufächern, wäre zum Scheitern verurteilt."

46 Vgl. auch die umfassenden Ausführungen bei Redeker in: Hoeren/Sieber/Holznagel (Hrsg.), MMR-HdB, Teil 12, Vertragsrecht für Internetdienste, passim; s. ferner Härting, Internetrecht, Kap. D. Verträge über Internetdienstleistungen, S. 203 ff. sowie Kosmides, Providing-Verträge, passim.

47 Bericht der Arbeitsgruppe „Digitaler Neustart“ der Konferenz der Justizministerinnen und Justizminister der Länder, S. 196, abrufbar a.a.O. (Fn. 43).

48 Hoblfeld/Godulla in: Hornung/Müller-Terpitz (Hrsg.), Rechtshandbuch Social Media, Kap. 2 Rn. 8. 
nen lassen können. ${ }^{49}$ Gleichwohl sind gewisse wiederkehrende Punkte erkennbar, denen der Charakter von Leitlinien für die Typologisierung zukommen kann. So handelt es sich bei einer Vielzahl der von den Providern angebotenen Nutzungsverträge um typengemischte Verträge. ${ }^{50}$ In der Literatur wurden dabei primär dienst-, werk- und mietvertragliche Elemente beziehungsweise deren unentgeltliche Entsprechung (i.e. Auftrag und Leihe) herausgearbeitet. ${ }^{51}$ Im Umgang mit typengemischten Verträgen ist mittlerweile anerkannt, dass sich die Bestimmung des anzuwendenden Rechts am Sinn und Zweck des Vertrages im Einzelfall zu orientieren hat. ${ }^{52}$ Insofern hat weder die Absorptionsmethode, ${ }^{53}$ nach welcher auf alle Vertragsklauseln das Recht des im Vordergrund stehenden Vertragstyps anzuwenden ist, noch die Kombinationsmethode, ${ }^{54}$ welche vorsieht, dass auf eine einzelne Klausel die jeweils für sie geltende Norm anzuwenden ist, grundsätzlich Vorrang. ${ }^{55}$ Stattdessen „ist bei der Beurteilung maßgeblich auf die besonderen Umstände des Einzelfalls, auf die Interessenlage der Vertragsparteien sowie auf Sinn und Zweck der vertraglichen Vereinbarungen abzustellen." ${ }^{66}$ Zur Bestimmung der Parteiinteressen und des Vertragszwecks ist das mehrseitige Finanzierungsmodell der Provider in den Blick zu nehmen, welches in Zusammenhang mit der Frage nach der (Un-)Entgeltlichkeit des Vertragsverhältnisses steht.

49 So etwa bei entstehenden Schnittstellen zu anderen Internetdiensten. Eine solche Schnittstelle kann eine Neubewertung des Nutzungsvertrags erforderlich machen, wenn dadurch Dienste unterschiedlicher Natur und Zielrichtung konvergieren, wie etwa bei Google Accounts, deren Funktionen laufend erweitert werden.

50 Bock, AcP (217) 2017, 370, 376 f.; Wehleit, MMR 2018, 279, 279 f.; Budzikiewicz, AcP (218) 2018, 558, 568 f.; Thiesen, Daten in der Erbmasse, S. 48.

51 Vgl. die Nachweise in Fn. 46; ferner Thiesen, Daten in der Erbmasse, S. 47 ff.; Kutscher, Der digitale Nachlass, S. 45 ff.; Seidler, Der digitale Nachlass, S. $62 \mathrm{ff}$. und $129 \mathrm{ff}$.

52 Vgl. nur Emmerich in: MüKo BGB, $\$ 311$ Rn. 28 m.w.N.

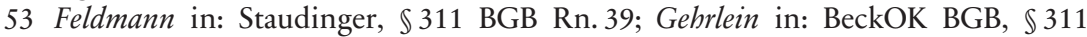
Rn. 21.

54 Feldmann in: Staudinger, $\mathbb{} 311$ BGB Rn. 40; Gehrlein a.a.O. (Fn. 53).

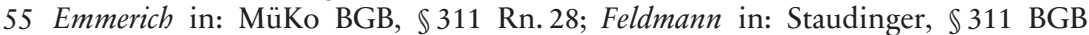
Rn. 41; Gehrlein a.a.O. (Fn. 53); Stadler in: Jauernig, \$311 BGB Rn. 33.

56 So BGH NJW 2008, 1072 Rn. 19; vgl. ferner bereits die Nachweise in Fn. 55. 


\section{Mehrseitiges Finanzierungsmodell der Provider}

Das denkbare Leistungsspektrum, zu dem sich der Provider im Rahmen des Nutzungsvertrags verpflichtet, wurde soeben skizziert. Nun ist ein Blick auf die nutzerseitigen Pflichten zu werfen. Vereinzelt besteht hier eine ausdrückliche Geldzahlungspflicht. Dies kann eine einmalige Zahlung zum Zeitpunkt der Anmeldung oder des Downloads (etwa der App des Anbieters) oder eine regelmäßig wiederkehrende Zahlung im Rahmen eines Abonnements sein. ${ }^{57}$ Zahlungspflichtige Dienste können die Premium-Versionen eines sonst nicht zahlungspflichtigen Dienstes sein und in dieser Version zusätzliche Funktionen oder zusätzliche Inhalte einschließen. ${ }^{58}$ In anderen Fällen werben kostenpflichtige Dienste damit, eine datenschutz-sensiblere Alternative zu ihren Mitbewerbern zu sein..$^{59}$ Damit verwandt ist das Angebot werbefreier Versionen mancher Dienste gegen Geldzahlung. ${ }^{60}$ Ausgeklammert sei an dieser Stelle die Frage nach In-AppKäufen und ihr Einfluss auf die Einordnung des Rahmenvertrags. ${ }^{61}$

Eine große Zahl der Nutzungsverträge wird jedoch geschlossen, ohne eine Geldzahlungspflicht des Nutzers vorzusehen. Mit dem sozialen Netzwerk Facebook und seinen verbundenen Anbietern WhatsApp und Instagram bietet ein Branchenriese ${ }^{62}$ den Nutzern seine Dienste ohne eine Geldzahlungspflicht als Gegenleistung an. Damit steht das Unternehmen exemplarisch für eine große Anzahl anderer Internetdiensteanbieter. Von dieser Tatsache sollte jedoch nicht voreilig auf die Unentgeltlichkeit dieser Vertragsbeziehungen geschlossen werden. Um der Komplexität des Phänomens gerecht zu werden, bedarf es eines Blicks auf das mehrseitige Finanzierungsverhältnis.

$57 \mathrm{Zu}$ kostenpflichtigen sozialen Netzwerken s. Redeker in: Hoeren/Sieber/Holznagel (Hrsg.), MMR-HdB, Teil 12 Rn. $420 \mathrm{f}$.

58 So z.B. Amazon Prime oder Tinder.

59 So z.B. Threema im Vergleich zu WhatsApp.

60 So z.B. Spotify Premium oder YouTube Premium.

61 Eine Analyse der Vertragsbeziehungen bei In-App-Käufen findet sich bei Kannowski/Till, NJOZ 2017, 522.

62 Facebook hatte im Dezember 2018 weltweit 1,52 Mrd. täglich und 2,32 Mrd. monatlich aktive Nutzer. In Deutschland waren es 23 Mio. täglich und 32 Mio. monatlich aktive Nutzer, s. PM des BKartA vom 7.02.2019, Bundeskartellamt untersagt Facebook die Zusammenführung von Nutzerdaten aus verschiedenen Quellen. 
a) Dienste gegen Daten

Die Anbieter finanzieren ihre Dienstleistung, indem sie Dritten anbieten, auf der Nutzeroberfläche des Internetdienstes Werbung zu schalten. ${ }^{63}$ Grundsätzlich ist es für Werbekunden attraktiv, wenn Werbung zielgruppenspezifisch platziert werden kann. ${ }^{64}$ Werden potenzielle Kunden adressiert, die sich ohnehin bereits für das Produkt des Werbenden interessieren oder zumindest in dessen Zielgruppe fallen, kann eine geringere Anzahl von Personen mit einer höheren Aussicht auf einen Geschäftsabschluss im Einzelfall beworben werden. Die Folge sind Effizienzsteigerungen. ${ }^{65} \mathrm{Die}$ Provider können diese Folge für ihre Werbeflächen ermöglichen, weil die Nutzer beim Abschluss des Nutzungsvertrages in die Erhebung und Speicherung ihrer personenbezogenen Daten zu Werbezwecken durch den Diensteanbieter einwilligen. Dies erlaubt den Providern das Anlegen von Nutzerprofilen, anhand derer wiederum die Werbekunden passgenau die Werbeadressaten aussuchen können. Damit wird die datenschutzrechtliche Einwilligung der Nutzer gem. Art. 6 Abs. 1 lit. a) DS-GVO ein Schlüsselelement in der Qualitätssicherung des Angebots der Provider gegenüber den Werbekunden. Letztere wiederum hoffen naturgemäß, ihre Werbeausgaben durch Geschäfte mit den adressierten Nutzern zu amortisieren. Dem Nutzungsvertrag liegt damit eine sogenannte Data-for-Access-Konstellation zugrunde.

63 Redeker, IT-Recht, Rn. 1278; ders. in: Hoeren/Sieber/Holznagel (Hrsg.), MMRHdB, Teil 12 Rn. 424; Schneider in: Redeker (Hrsg.), IT-Verträge, Kap. 3.7. In den Nutzungsbedingungen von Facebook heißt es dazu im zweiten Absatz: Anstelle einer Zahlungspflicht der Nutzer „bezahlen uns Unternehmen und Organisationen dafür, dass wir dir Werbeanzeigen für ihre Produkte und Dienstleistungen zeigen. Durch die Nutzung unserer Produkte erklärst du dich damit einverstanden, dass wir dir Werbeanzeigen zeigen dürfen, die nach unserer Ansicht relevant für dich sind und deinen Interessen entsprechen. Wir verwenden deine personenbezogenen Daten, um festzulegen, welche Werbeanzeigen wir dir zeigen.“, abrufbar unter https:/www.facebook.com/legal/terms/update [17.11.2020].

64 Mundt in: PM des BKartA vom 7.02.2019, Bundeskartellamt untersagt Facebook die Zusammenführung von Nutzerdaten aus verschiedenen Quellen.

65 Dazu und zu weiteren Vorteilen der personalisierten Werbung im Internet siehe Schneider in: Redeker (Hrsg.), IT-Verträge, Kap. 3.7 Rn. 1a; Paal, GRUR 2013, 873, 876 beschreibt diese Konstellation im Kontext von Suchmaschinen. 


\section{b) Unentgeltlichkeit des Nutzungsvertrags}

Es ist zu klären, ob aus der Tatsache, dass der Nutzer den Provider mit seinen Daten für die Diensterbringung „,bezahlt“, die Entgeltlichkeit des Nutzungsvertrags folgt. Dazu müsste die Einwilligung in die Nutzung der personenbezogenen Daten die synallagmatische Gegenleistung für die Nutzungsgewährung des Internetdienstes sein. ${ }^{66}$ Zunächst spricht der Grundsatz der Relativität der Schuldverhältnisse dagegen, die soeben skizzierten Beobachtungen zur Mehrseitigkeit des Social-Media-Marktes in die Beurteilung des spezifischen Vertragsverhältnisses zwischen Provider und Nutzer miteinzubeziehen. Denn grundsätzlich sind die Rechtswirkungen eines Schuldverhältnisses auf die an ihm Beteiligten beschränkt. ${ }^{67}$ Die Rechtsstellung Dritter - im Verhältnis zwischen Provider und Werbekunden also die Rechtsstellung der Nutzer - wird durch das Schuldverhältnis in der Regel nicht berührt. ${ }^{68}$ Gleichwohl kann die Interessenlage der Parteien bei Vertragsschluss durchaus ein Indiz für die Beurteilung des Vertragsverhältnisses sein. Denn es entspräche wohl zumindest dem Interesse des Providers, wenn die nutzerseitige Einwilligung in die Datenverarbeitung vertraglich bindend wäre und sich der Grundsatz pacta sunt servanda darauf erstrecken würde. ${ }^{69}$ Das würde für den Provider zu Planungssicherheit bei der Vermarktung der personalisierten Werbeflächen führen. Dagegen spricht jedoch die freie Widerrufbarkeit der datenschutzrechtlichen Einwilligung gem. Art. 7 Abs. 3 DS-GVO, mit welcher der unionale Gesetzgeber der Bedeutung des Grundrechts auf Schutz personenbezogener Daten im Sinne von Art. 8 Abs. 1 und 2 S. 1 GRCh Rechnung trägt. ${ }^{70}$

Dadurch wird die Auseinandersetzung über die Entgeltlichkeit des Nutzungsvertrags eine spezifisch datenschutzrechtliche - und damit spätestens seit Geltung der DS-GVO eine europarechtliche - Frage. Sofern also Bräu-

66 So ausdrücklich Bräutigam, MMR 2012, 635, $638 \mathrm{f}$., der darin wahlweise einen Tauschvertrag oder einen Vertrag sui generis sieht; s. auch Wehleit, MMR 2018, 279, 279 f. m.w.N.; a.A. Hacker, ZfPW 2019, 148, $172 \mathrm{ff}$.

67 Ernst in: MüKo BGB, Einleitung Schuldrecht, Rn. 18 ff.; Bachmann in: MüKo BGB, $\mathbb{2} 241$ Rn. 11; Mansel in: Jauernig, BGB, $\$ 241$ Rn. 4.

$68 \mathrm{Zu}$ den Ausnahmen, die hier allesamt nicht einschlägig sind, s. Mansel in: Jauernig, BGB, $\$ 241$ Rn. 6 m.w.N.

69 Bräutigam, MMR 2012, 635, 636; s. aber Hacker, ZfPW 2019, 148, 170 der die Providerinteressen auch bei jederzeitiger Widerrufbarkeit nicht gefährdet sieht.

70 Frenzel in: Paal/Pauly (Hrsg.), DS-GVO, Art. 7 Rn. 16. Zum Verhältnis von schuldrechtlicher (Selbst)bindung und freier Widerruflichkeit der Einwilligung siehe auch Zech, GRUR 2015, 1151, $1154 \mathrm{f}$. 
tigam dafür argumentiert, dass die datenschutzrechtliche Einwilligung gleichsam unter Anpassung ihrer Rechtsnatur von einer lediglich datenschutzrechtlichen Einwilligung zu einer vertraglichen Verpflichtung jenseits der Anwendbarkeit des Datenschutzrechts umgedeutet werden soll, ${ }^{71}$ so ist das mit dem Geltungsanspruch der DS-GVO kaum vereinbar. ${ }^{72}$ Die DS-GVO ist nach Maßgabe ihres Art. 2 DS-GVO auf die Verarbeitung von personenbezogenen Daten durch die Anbieter digitaler Informations- und Kommunikationsdienste anwendbar. Die Rechtmäßigkeit der Verarbeitung wird in der Folge durch Art. 6 DS-GVO bestimmt und kann nicht durch das nationale Recht des BGB überformt werden. Etwas anderes mag allenfalls gelten, wenn einzelne Normen des BGB der Umsetzung europarechtlicher Vorgaben dienen und insofern selbst Ausprägungen von Europarecht sind. Zwar lässt der Widerruf die Zulässigkeit der Datenverarbeitung aufgrund anderer Zulässigkeitsgründe aus Art. 6 Abs. 1 lit.b)-f) DSGVO unberührt. ${ }^{73}$ Nähme man jedoch an, dass sich die Zulässigkeit der Datenverarbeitung (auch nach einem Widerruf) sodann aus Art. 6 Abs. 1 lit. b) DS-GVO ergäbe, so liefe die Anordnung ,jederzeitiger“ Widerrufbarkeit aus Art. 7 Abs. 3 S. 1 DS-GVO leer. Nach geltendem Recht kommt die Nutzung personenbezogener Daten als Gegenleistung im vertraglichen Synallagma daher nach hier vertretener Ansicht nicht in Betracht. ${ }^{74}$ Demnach handelt es sich bei Nutzungsverträgen de lege lata um unentgeltliche Vertragsverhältnisse. Als rechtspolitischer Ausblick sei aber darauf verwiesen, dass der nationale Gesetzgeber die Vorgaben der europäischen Richtli-

71 Bräutigam, MMR 2012, 635, 638 ff., allerdings noch zur Rechtslage vor Geltung der DS-GVO; sich dem anschließend Kutscher, Der digitale Nachlass, S. 45 f.

72 Entsprechend argumentiert Klement in: NK-Datenschutzrecht, Art. 7 DS-GVO Rn. 92 auch spezifisch europarechtlich, wenn er sich Bräutigam anschließt. Der Grundsatz der Vertragsfreiheit sei auch europarechtlich anerkannt. Daher sei in dieser Konstellation von einem wirksamen vertraglichen Ausschluss des Widerrufs auszugehen. Es sei treuwidrig, eine Einwilligung zu widerrufen, die wegen einer wirksamen vertraglichen Verpflichtung sofort wieder erteilt werden müsste (dolo-agit-Einwand); s. aber Frenzel in: Paal/Pauly (Hrsg.), DS-GVO, Art. 6 Rn. 13; wie hier Hacker, ZfPW 2019, 148, 169 f.; Spindler/Dalby in: Spindler/Schuster (Hrsg.), Recht der elektronischen Medien, Art. 7 DS-GVO Rn. 12 f.

73 Frenzel in: Paal/Pauly (Hrsg.), DS-GVO, Art. 7 Rn. 17a.

74 A.A. Bock, AcP (217) 2017, 370, 377; Specht-Riemenschneider in: Röhricht/Graf von Westphalen/Haas (Hrsg.), HGB, Plattformnutzungsverträge, Rn. 20 f.; Spindler/ Dalby in: Spindler/Schuster (Hrsg.), Recht der elektronischen Medien, Art. 7 DSGVO Rn. 13 wollen eine Kollision von Schuld- und Datenschutzrecht verhindern, indem der datenschutzrechtliche Widerruf stets zugleich als schuldrechtliche Kündigung aufgefasst wird. 
nie über bestimmte vertragsrechtliche Aspekte der Bereitstellung digitaler Inhalte und digitaler Dienstleistungen ${ }^{75}$ wird umsetzen müssen. ${ }^{76}$

\section{Zwischenergebnis zur Vertragstypologie von Nutzungsverträgen}

Sofern sie die Entgeltlichkeit des Vertragsverhältnisses - wie hier - ablehnen, schlagen einige Stimmen in der Literatur die Annahme eines Auftrags im Sinne von $\$ 662$ BGB vor. ${ }^{77}$ Das ist aus mehreren Gründen aber ebenfalls unpassend. Kern des Auftragsverhältnisses ist eine fremdnützige Tätigkeit - nämlich die unentgeltliche Besorgung eines Geschäfts durch den Beauftragten. ${ }^{78}$ Der Betreiber des sozialen Netzwerks wird aber - wie gesehen - gerade in seinem eigenen (ökonomischen) Interesse tätig. Ferner dürften die gesetzlich vorgesehenen Ansprüche des Auftragsrechts den Interessen beider Vertragsparteien zuwiderlaufen. Der Provider wird, als Beauftragter, weder die von ihm erstellten Nutzerprofile noch gar seine Werbeeinnahmen gem. $\$ 667$ BGB als das durch die Geschäftsbesorgung Erlangte herausgeben. Der Nutzer wird als Auftraggeber keinen Aufwendungsersatz gem. $\$ 670$ BGB für die IT-Infrastruktur des Providers leisten. ${ }^{79}$

Nach alledem einigen sich die Nutzungsvertragsparteien regelmäßig auf einen Vertrag sui generis, der für den Nutzer unentgeltlich ist und je nach

75 Im Vergleich zur verabschiedeten Fassung sah die Entwurfsfassung der Richtlinie Daten noch eindeutiger als Gegenleistung im vertraglichen Synallagma vor, vgl. noch Art. 3 Nr. 1 RL-E: „Diese Richtlinie gilt für alle Verträge, auf deren Grundlage ein Anbieter einem Verbraucher digitale Inhalte bereitstellt oder sich hierzu verpflichtet und der Verbraucher als Gegenleistung einen Preis zahlt oder aktiv eine andere Gegenleistung als Geld in Form personenbezogener oder anderer Daten erbringt." (Hervorhebung nicht im Original) Vgl. dazu Spindler, MMR 2016, 147, 147 ff.; s. ferner Mischau, ZEuP 2020, 335.

76 Zur Fortentwicklungsperspektive durch den europäischen Gesetzgeber s. Paal/ Hennemann, NJW 2017, 1697, 1698 m.w.N.; Metzger, AcP 216 (2016), 817, passim; zum Ganzen sehr ausführlich und mit rechtspolitischer Handlungsempfehlung an den Gesetzgeber s. Bericht der Arbeitsgruppe „Digitaler Neustart“ der Konferenz der Justizministerinnen und Justizminister der Länder, S. 199 ff., abrufbar a.a.O. (Fn. 43).

77 Redeker in: Hoeren/Sieber/Holznagel (Hrsg.), MMR-HdB, Teil 12 Rn. 422 ff., 428; Seidler, Digitaler Nachlass, S. $129 \mathrm{ff}$.

78 Schäfer in: MüKo BGB, $\mathbb{S} 662$ Rn. 1; D. Fischer in: BeckOK BGB, $\$ 662$ Rn. 1.

79 Bräutigam, MMR 2012, 635, 636; das Problem sieht auch Redeker, IT-Recht, Rn. 1279, der darüber allerdings hinweggeht und trotzdem von einem Auftrag ausgeht, weil mangels Bestimmtheit „vermutlich keine konkreten Ansprüche bestehen." 
genauer Ausgestaltung des Internetdienstes dienst-, werk- und/oder mietvertragliche Leistungspflichten des Providers vorsieht. ${ }^{80}$

\section{Urheber- und Leistungsschutzrechte des Erblassers}

Unabhängig von der lediglich nutzungsvertraglichen Rechtsposition an dem gesamten Account und seinen Inhalten können dem Nutzer absolute Rechte nach Maßgabe des UrhG an einzelnen Accountinhalten zustehen: ${ }^{81}$ Urheber- bzw. Leistungsschutzrechte an den vom Nutzer selbstgenerierten Inhalten. ${ }^{82}$ Der Wert und die Bedeutung dieser Rechtsposition kann nur im Einzelfall bestimmt werden und variiert nach dem Nutzungsverhalten des Erblassers. Angesichts großer - mitunter sogar unbegrenzter - Speicherkapazitäten, die dem Nutzer vom Provider zur Verfügung gestellt werden, besteht aber jedenfalls das Potenzial einer Vielzahl von urheberrechtlich relevanten Accountinhalten. ${ }^{83}$

Gem. $\ 28$ Abs. 1 UrhG ist das Urheberrecht vererblich. ${ }^{84}$ Der Übergang dieser Rechtsposition im Erbfall erfolgt grundsätzlich unabhängig vom Schicksal des Nutzungsvertrags. Insbesondere etwaige datenschutz- und telekommunikationsrechtliche Beschränkungen des Erbrechts an der nutzungsvertraglichen Position, die nicht zuletzt dem Schutz der Kommunikationspartner des Erblassers dienen, ${ }^{85}$ spielen mit Blick auf übergehende Urheber- und Leistungsschutzrechte keine Rolle. Dasselbe gilt für einen (formularvertraglichen) Ausschluss der Vererbbarkeit des Accounts, ${ }^{86}$ der sich, seine Wirksamkeit vorausgesetzt, ausschließlich auf die nutzungsvertragliche nicht aber auf die urheberrechtliche Rechtsposition des Erblassers auswirken kann.

80 Siehe dagegen aber Schäfer in: MüKo BGB, $\$ 662$ Rn. 18 a.E.

81 A.A. Seidler, Digitaler Nachlass, S. 73 f.

82 Mit Beispielen Bullinger in: Wandtke/Bullinger (Hrsg.), UrhG, $\mathbb{2}$ Rn. $156 \mathrm{ff}$; Bauer, User Generated Content, in: Große Ruse-Khan/Klass/v. Lewinski (Hrsg.), Nutzergenerierte Inhalte als Gegenstand des Privatrechts, S. 6 ff.; Reinemann/Remmertz, ZUM 2012, 216, $218 \mathrm{ff}$.

83 Vgl. dazu noch ausführlich auf S. $189 \mathrm{ff}$.

84 Dasselbe gilt für den Leistungsschutz an Licht- und Laufbildern, s. Obly in: Schricker/Loewenheim (Hrsg.), $\$ 28$ UrhG Rn. 18, 20; Nordemann in: Fromm/Nordemann, $\$ 28$ UrhG Rn. 16; Rehbinder/Peukert, Urheberrecht, Rn. 991.

85 Vgl. dazu noch ausführlich auf S. $94 \mathrm{ff}$.

86 Siehe dazu S. $166 \mathrm{ff}$. 
Gleichwohl ist ein enger Zusammenhang zwischen dem Account und seinen Inhalten nicht abzustreiten. Sehen etwa die Nutzungsbedingungen eines Anbieters von Cloud-Speicherplatz im Falle des Todes des Nutzers die Löschung des Accounts mitsamt seinen Inhalten vor, ${ }^{87}$ kann das erhebliche (faktische) Auswirkungen für ein urheberrechtlich geschütztes Werk haben, wenn und soweit keine weiteren Kopien (beispielsweise eines Textmanuskripts oder von Fotografien) außerhalb des Accounts existieren. Ein weiterer Berührungspunkt der vertraglichen und der absoluten Rechtsposition ist die Einräumung von Urheberrechtslizenzen durch den Nutzer, auf welche der Provider angewiesen ist, um die geschützten Inhalte auf einem Server speichern und Dritten, wie den Privatkontakten oder Followern des Nutzers, zugänglich machen zu dürfen. ${ }^{8}{ }^{8} \mathrm{Im}$ Erbfall ist die Bindung der Rechtsnachfolger an diese Lizenzen im Einzelfall zu prüfen, wobei mit Blick auf den Zweck der Lizenzierung auch das postmortale Schicksal des Nutzungsvertrags zu berücksichtigen ist. ${ }^{89}$

Im weiteren Verlauf der Untersuchung werden die Immaterialgüterrechte des Erblassers als eigenständige Erbrechtsobjekte zu beleuchten sein. Gleichzeitig sind sie auch und gerade in ihren Bezügen zu den Nutzerkonten des Erblassers bei Anbietern von Informations- und Kommunikationsdiensten zu betrachten. Denn aus der gesamten Schnittmenge der Rechtsgebiete „Urheberrecht und Erbrecht ${ }^{“ 90}$ sind hier diejenigen Aspekte herauszugreifen, die den Lebensbereich eines durchschnittlichen Nutzers sozialer Medien und damit seinen digitalen Nachlass betreffen.

87 So z.B. Apple bezüglich der Apple-ID für iCloud, vgl. Ziff. IV. D. der AGB, abrufbar unter https://www.apple.com/legal/internet-services/icloud/de/terms.html [17.11.2020]. Siehe dazu ein Versäumnisurteil des LG Münster ErbR 2019, 455 (mit Anm. von Pruns) zulasten von Apple.

88 Betroffen sind mindestens $\mathbb{S} \mathbb{S} 16,19 \mathrm{a}$ und ggf. 23 S. 1 UrhG, s. Berberich, MMR 2010, 736, 737, 739; Paul in: Hoeren/Sieber/Holznagel (Hrsg.), MMR-HdB, Teil 7.4 Rn. 140; Solmecke ebd., Teil 21.1 Rn. 6; ders./Dam, MMR 2012, 71, 72; ferner König/Stang in: Münchener Anwaltshandbuch Urheberrecht, $\$ 31$ Rn. 14.; zweifelnd aber Imhof in: Bisges (Hrsg.), Handbuch Urheberrecht, Kap. 4 Rn. 139.

89 Zum Ganzen s. S. $194 \mathrm{ff}$.

90 Monografisch dazu insgesamt Clément, Urheberrecht und Erbrecht, und Gloser, Die Rechtsnachfolge in das Urheberrecht, jeweils passim; überblicksartig zur einschlägigen Rechtsprechung Gergen, ZErb 2009, 42, 42 ff. 
III. Zwischenergebnis zu A.

Mit der großen Vielfalt der angebotenen Internetdienste korreliert eine Vielgestaltigkeit der Nutzungsverträge. Das betrifft sowohl das Leistungsspektrum des Providers, welches von der technisch-funktionalen Ausgestaltung des Internetdienstes abhängt, als auch die Frage nach einer etwaigen Gegenleistung des Nutzers. Letztere kann in einer Geldleistung oder in der Bereitschaft bestehen, sich durch die Preisgabe persönlicher Daten als „(werbe)vermarktetes Produkt“91 des Providers gegenüber dessen Werbekunden zur Verfügung zu stellen. Vor diesem Hintergrund bleibt zwar eine einzelfallbezogene Beurteilung unumgänglich. Jedenfalls werden aber eine Vielzahl der Nutzungsverhältnisse als unentgeltliche Verträge eigener Art mit dienst-, werk- und/oder mietvertraglichen Elementen abgeschlossen. In diesem Rahmen hält der Provider einen Account bereit, in welchen der Nutzer unterschiedliche Inhalte einbringen kann. Etwaige an diesen Inhalten bestehende Urheber- und Leistungsschutzrechte sowie die vertragliche Rechtsposition des Nutzers bilden als Erbrechtsobjekte den Gegenstand dieser Untersuchung.

\section{B. Daten als solche - (k)ein Recht an Daten}

Die Untersuchung des Erbrechtsobjekts im Rahmen des digitalen Nachlasses berührt eine weitere Debatte, die ebenfalls im Bereich der Schnittstelle zwischen Digitalisierung und (Privat)recht geführt wird: In der rechtswissenschaftlichen Literatur wird über ein sogenanntes Recht an Daten diskutiert. ${ }^{92}$ Dabei stehen zwei verwandte Fragen im Zentrum. Zum einen wird das geltende Recht daraufhin untersucht, ob es ein eigentumsähnliches Ausschließlichkeitsrecht an Daten gibt oder ob ein entsprechendes Recht mittels Analogiebildung konstruiert werden kann. ${ }^{93}$ Zum anderen wird in Abhängigkeit von der Beantwortung der ersten Frage - eine rechtspolitische Debatte darüber geführt, ob es sinnvoll und notwendig ist, ein solches Recht neu zu schaffen. ${ }^{94}$ Die Auseinandersetzungen sollen hier (nur)

91 So im Kontext von $\$ 18$ Abs. 2a GWB Paal, WuW 2016, 453.

92 Grundlegend Zech, Information als Schutzgegenstand, passim; Amstutz, AcP 218 (2018), 439, 439 ff.; überblicksartig Boehm, ZEuP 2016, 358, 381 ff. und Röttgen in: Specht-Riemenschneider/Werry/Werry (Hrsg.), Datenrecht in der Digitalisierung, S. 373 ff.; vgl. ferner die Nachweise in den folgenden Fußnoten.

93 Hierzu sogleich auf S. $48 \mathrm{ff}$.

94 Vgl. dazu S. 66 f. 
insoweit dargestellt werden, wie sie für die erbrechtliche Untersuchung dieser Arbeit fruchtbar gemacht werden können. Denn wenn und soweit ein entsprechendes Recht an Daten besteht oder eingeführt wird, kann es als Rechtsobjekt in den digitalen Nachlass fallen. ${ }^{95}$ Allerdings begegnet die juristische Konstruktion eines Rechts an Daten technischen wie juristischen Bedenken.

\section{Einführung in die Debatte}

Ausgangspunkt der Debatte ist die Annahme, Daten seien „der Rohstoff der Zukunft" ${ }^{* 96}$. Mit diesem Bild geht ein großes Interesse an der Frage nach den Herrschaftsrechten über diesen „Rohstoff“ einher. ${ }^{97}$ Gleichzeitig stellen Daten mit ihren technischen Spezifika - insbesondere der Immaterialität, der einfachen Möglichkeit der Vervielfältigung ohne Qualitätseinbuße sowie rapide wachsenden Speicherkapazitäten - gegenüber sonstigen Gütern eine eigene Kategorie dar. ${ }^{98}$ Es erscheint fraglich, ob und inwieweit die Zuordnung von Daten durch traditionelle Rechtsmaterien erfolgen kann, deren originärer Regelungsgegenstand (wie etwa im Falle des Sachen- oder Immaterialgüterrechts) ein anderer ist.

Vor diesem Hintergrund ist auch der Begriff des „Dateneigentums“, unter dem die Debatte oder einzelne ihrer Aspekte mitunter geführt werden, kritisch zu würdigen. ${ }^{99}$ Die begriffliche Anlehnung an das Sachenrecht

95 Thiesen, Daten in der Erbmasse, S. 181 hält es für möglich, dass eine „Materialisierung von Kommunikationsinhalten [...] potenziell die Behandlung des digitalen Nachlasses vereinfachen" kann; vgl. auch Stieper, der fragt, ob neben vertraglichen Abwicklungsansprüchen nicht auch über eine Verdinglichung von Daten im digitalen Nachlass nachgedacht werden müsse, zitiert nach dem Diskussionsbericht von Bornhauser, AcP (218) 2018, 594, $597 \mathrm{f}$.

96 So Bundeskanzlerin Angela Merkel zitiert nach Markendorf, ZD 2018, 409, 409; Kühling/Sackmann, ZD 2020, 24, 24; sowie Paal/Hennemann, NJW 2017, 1697, 1697 f., die das Bild „wegen der wettbewerblichen Eigenheiten von Daten [als] etwas schief" bewerten: Daten sind anders als andere (insbesondere körperliche) Güter im Konsum nicht-rival, sie sind nicht-abnutzbar und idR. nicht-exklusiv. S. dazu auch die Nachweise in Fn. 98 und 115.

97 Markendorf, ZD 2018, 409, 409; Zech, GRUR 2015, 1151, 1152 sowie ders., CR 2015, 137, 139 m.w.N.

98 Zech, CR 2015, 137, 140.

99 Kühling/Sackmann, Rechte an Daten, Regulierungsbedarf aus Sicht des Verbraucherschutzes?, 20. November 2018, Rechtsgutachten im Auftrag des Verbraucherzentrale Bundesverband, abrufbar unter https:/www.vzbv.de/pressemitteilung/m eine-daten-gehoeren-dir, S. 6, 7 f., [17.11.2020]. 
veranschaulicht, dass unter dem Schlagwort den Möglichkeiten (und Grenzen) eines Zuordnungsregimes für Daten nachgegangen wird. ${ }^{100}$ Das ist zwar in funktionaler Hinsicht nicht falsch, insbesondere wenn ein umfassendes Herrschaftsrecht an Daten untersucht bzw. gefordert wird. Dogmatisch hingegen impliziert „Dateneigentum“ fälschlicherweise die Gleichsetzung der Rechtsinstitute von Sacheneigentum und einem etwaigen Recht an Daten. ${ }^{101}$ Das kann durch die Termini „Datenausschließlichkeitsrecht" oder (eigentumsähnliches) „Recht an Daten“ vermieden werden. Die Bezeichnung Recht an Daten bildet ferner die Breite der geführten Auseinandersetzung besser ab, da neben umfassenden Herrschaftsrechten auch (einfache) Nutzungs- und/oder Zugangsrechte thematisiert werden. ${ }^{102}$ Wenn im Folgenden der Begriff „Dateneigentum“ trotzdem gebraucht wird, so geschieht das in dessen Qualität als Schlagwort zur Bezeichnung der gesamten Debatte über einen datenrechtlichen Ordnungsrahmen.

In dieser Debatte lassen sich grob zwei Stoßrichtungen unterscheiden, die zwar verwandt sind und Parallelen aufweisen, aber durch einen jeweils eigenen (rechts)politischen Impetus gekennzeichnet sind und auch teilweise unterschiedliche Anwendungsfelder etwaiger Datenrechte vor Augen haben. Zum einen gibt es die primär aus Sicht des Individuums konzeptionierte Forderung, die Herrschaft einer Privatperson über „ihre eigenen Daten " $\mathrm{zu}$ gewährleisten. ${ }^{103} \mathrm{Zum}$ anderen werden Datenrechte im Zusammenhang mit der kommerziellen, maschinengesteuerten Auswertung sehr großer Datenmengen (sog. Big Data) untersucht und hier oft im Zusam-

100 Ebd. Fn. 99.

101 Das Sachenrecht kann aber mangels Körperlichkeit von Daten nicht zur Begründung eines „Dateneigentums“ herangezogen werden. Siehe dazu S. $48 \mathrm{f}$. So auch die einhellige Meinung, s. die Nachweise in Fn. 113 und 116.

102 Ebd. Fn. 99.

103 In diesem Sinne beispielsweise Fezer, ZD 2017, 99, $99 \mathrm{ff}$. sowie ders., MMR 2017, 3, passim, der ein „immaterialgüterrechtliches Eigentum an verhaltensgenerierten Personendaten der Nutzer als Datenproduzenten" fordert. 


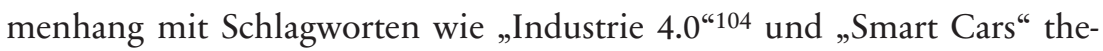
matisiert. ${ }^{105}$

Für den digitalen Nachlass sind jedwede Datenrechte potenziell von Interesse, wenn und soweit sie als Aktiva oder auch als Passiva in den Nachlass fallen (können). Im Kontext sozialer Medien werden dabei vor allem die Rechte an solchen Datensätzen relevant sein, die entweder einen Personenbezug im Sinne des Datenschutzrechts haben oder aus anderen Gründen einen persönlichkeitsrechtlichen Schutz genießen. ${ }^{106}$ Als ein Beispiel sei hier die Frage nach Ausschließlichkeitsrechten an Daten im Rahmen von Cloud-Computing im B2C-Bereich genannt. ${ }^{107}$

104 Nach Zech, GRUR 2015, 1151, 1152 m.w.N. muss im Rahmen der „Industrie 4.0“ zwischen drei Entwicklungen differenziert werden: Erstens werden körperliche Güter mit Datenbezügen ausgestattet (sog. „Smart Products“; bspw. Smartphone, „Smart Cars“). Zweitens werden körperliche Güter durch Daten ersetzt (sog. „e-products“; bspw. E-Books, Streaming). Drittens werden neuartige Güter eigener Art gehandelt - über datengetriebene Wirtschaft hinaus entsteht infolgedessen eine Wirtschaft mit Daten. Daten werden damit zu einem Wirtschaftsgut, das in einer Datenwirtschaft gehandelt wird.; zur wettbewerbsrechtlichen Dimension Paal/Hennemann, NJW 2017, 1697, $1698 \mathrm{f}$.

105 Vgl. Grützmacher, CR 2016, 485, 485 ff.; aus ökonomischer Perspektive Kerber, GRUR Int. 2016, 989, 990; in diesem Kontext auch thematisiert von Weidert/Bug in: Röhricht/Graf von Westphalen/Haas (Hrsg.), HGB, Zulieferverträge in der Industrie 4.0, Rn. 24 ff.; Ensthaler, NJW 2016, 3473, 3473 ff.; nach Kühling/Sackmann, Rechte an Daten, Regulierungsbedarf aus Sicht des Verbraucherschutzes?, abrufbar a.a.O. Fn 99, S.6, war der Umgang mit Daten aus vernetzten Fahrzeugen sogar der Ausgangspunkt der aktuellen Debatte. Die deutsche Automobilindustrie sei bestrebt, in einer zunehmend datengetriebenen Mobilität weiterhin zentraler Teil der Wertschöpfungskette zu sein und nicht etwa reiner Zulieferer von Digitalkonzernen. In Ansehung der starken deutschen Automobilindustrie bei einer - im Vergleich zu den USA - wenig entwickelten Datenwirtschaft bestehe daran in Deutschland ferner ein industriepolitisches Interesse. So verwundere es nicht, dass eine regulatorische Neuordnung bei Fragen nach der Zuordnung von Daten auch und gerade im Mobilitätsumfeld aufgeworfen werde.

106 Nicht-personenbezogene Daten, auf die das Datenschutzrecht keine Anwendung findet und die auch sonst nicht persönlichkeitsrechtlich relevant sind, werden in Abgrenzung dazu als Rohdaten bezeichnet, s. etwa Zech, GRUR 2015, 1151, 1152; Kerber, GRUR Int. 2016, 989, 990 f. spricht von ,industrial data"; kritisch zu dieser Bezeichnung Hoeren/Pinelli, JZ 2020, 879, 879 in Fn. 1.

$107 \mathrm{Zu}$ der Frage, ob in diesem Fall Ausschließlichkeitsrechte an den Daten in der Cloud bestehen und, bejahendenfalls, ob diese Rechte dem Cloud-Anbieter bzw. dem Nutzer zustehen, vgl. Boehm, ZEuP 2016, 358, 379 ff. 
Im Folgenden soll zunächst ein Blick auf die Situation de lege lata geworfen werden (dazu II.), bevor sodann in einem Ausblick die rechtpolitische Diskussion zu beleuchten ist (dazu III.).

\section{Konstruktionsvorschläge nach geltendem Recht}

Ein explizit (spezial-)gesetzlich geregeltes Ausschließlichkeitsrecht an Daten als solchen besteht im geltenden Recht nicht. Freilich können einzelne immaterialgüterrechtlich geschützte Positionen mit Datenbezug bestehen. Dafür steht exemplarisch der urheberrechtliche Schutz von Software nach $\$ \$$ 69a ff. UrhG, dessen Grundlage aber der Werkcharakter des Computerprogramms und nicht etwa die Speicherungsform der Software ist. ${ }^{108}$ Weder die digitale Form an sich noch der Akt der Datenerzeugung selbst sind im geltenden Recht der (entscheidende) Anknüpfungspunkt für ein Ausschließlichkeitsrecht an Daten. Zur Konstruktion etwaiger Rechte an Daten kommt daher allenfalls die entsprechende Anwendung anderer Regelungen in Betracht.

\section{Sachenrecht}

Zunächst drängt sich die Untersuchung der Anwendbarkeit sachenrechtlicher Grundsätze auf. Das liegt nicht zuletzt an dem Schlagwort des „Dateneigentums", unter dem die Debatte vielfach geführt wird. Darüber hinaus liegt für Fragen nach der Güterzuordnung sowie der Zuweisung absoluter Rechtspositionen auch in funktionaler Hinsicht ein Blick in das dritte Buch des BGB nahe. Als Ausgangspunkt der sachenrechtlichen Kategorien von Besitz und Eigentum verweist das Gesetz in den $\$ \$ 854$ Abs. 1 und 903 S. 1 BGB auf den Begriff der Sache als das maßgebliche Rechtsobjekt. Nach $\$ 90$ BGB sind Sachen im Sinne des Gesetzes nur körperliche Gegenstände. Körperlich ist ein Gegenstand, wenn man ihn anfassen oder zumindest sinnlich wahrnehmen und beherrschen kann. ${ }^{109}$ In technischer Hinsicht handelt es sich bei gespeicherten Daten um ein bestimmtes Mus-

108 Vgl. $\$ 69$ a Abs. 3 UrhG, dazu Dreier in: Dreier/Schulze (Hrsg.), UrhG, $\$ 69 a$ Rn. 1 und ferner Wiebe in: Spindler/Schuster (Hrsg.), Recht der elektronischen Medien, $\$$ 69a UrhG Rn. $26 \mathrm{ff}$.

109 Stresemann in: MüKo BGB, $\mathbb{9} 90$ Rn. 1; Dörner in: HK-BGB, $\mathbb{9} 90$ BGB Rn. 2 f.; zum Sachbegriff insgesamt auch Mansel in: Jauernig, BGB, Vor $\$ \$ 90$ ff. Rn. 2 ff. 
ter magnetischer Spannung, ${ }^{110}$ das nicht ohne Zuhilfenahme technischer Infrastruktur wahrnehmbar ist. ${ }^{111}$ Daten sind - anders als Speichermedien wie etwa Festplatten ${ }^{112}$ - mangels Körperlichkeit keine Sachen im Sinne des BGB. ${ }^{113}$

Außerdem fehlt es Daten regelmäßig ${ }^{114}$ auch an der für das Sacheigentum charakteristischen Rivalitätsbedingung: Daten können von mehreren Personen simultan genutzt und mit nur geringem Aufwand ohne Verlust vervielfältigt werden. ${ }^{115}$ An Daten kann folglich weder Sacheigentum noch Besitz im Sinne der $\$ \$ 903$ S. 1 und 854 Abs. 1 BGB bestehen. ${ }^{116}$ Umgekehrt ändert sich auch die Eigentumslage an einem Datenträger nicht dadurch, dass auf ihm bestimmte Daten gespeichert werden, solange der Datenträger selbst dabei nicht seine typische Funktion verändert und auf diese Weise zu einer neuen Sache im Sinne von $\$ 950$ Abs. 1 S. 2 BGB wird. ${ }^{117}$ Eine solche Veränderung wird bei praxisnaher Betrachtung regelmäßig nicht gegeben sein.

110 Wagner in: MüKo BGB, $\mathbb{8} 823$ Rn. 245. Siehe auch Bericht der Arbeitsgruppe „Digitaler Neustart“ der Konferenz der Justizministerinnen und Justizminister der Länder, S. 32 f., abrufbar a.a.O. (Fn. 43).

111 Berberich, Virtuelles Eigentum, S. $90 \mathrm{f}$.

112 Stieper in: Staudinger, $\mathbb{\$} 90$ BGB Rn. 12; Stresemann in: MüKo BGB, $\mathbb{9} 90$ Rn. 25; Wagner in: MüKo BGB, $\$ 823$ Rn. 246.

113 Boehm, ZEuP 2016, 358, 381; Amstutz, AcP 218 (2018), 439, 544 gegen Berberich, Virtuelles Eigentum, S. 92 f.; nach Stieper in: Staudinger, $\mathbb{} 90$ BGB Rn. 18 folge auch bei unterstellter Sachqualität nichts anders, da Daten in diesem Fall wesentliche Bestandteile des Datenträgers darstellten und nach $₫ 93$ BGB nicht sonderrechtsfähig wären. A.A. wohl Mansel in: Jauernig, BGB, Vor $\$ \$ 90 \mathrm{ff}$. Rn. 3.

114 Berberich, Virtuelles Eigentum in: Große Ruse-Khan/Klass/v. Lewinski (Hrsg.), Nutzergenerierte Inhalte als Gegenstand des Privatrechts, S. 181 ff.; rechtspolitisch dazu, wie man mittels Blockchain-Technologie zumindest zu einer (rechtlichen) Erstellungsvermutung gelangen kann, s. Markendorf, ZD 2018, 409, $411 \mathrm{ff}$.

115 Wagner in: MüKo BGB, $\mathbb{8} 823$ Rn. 335; Zech, Information als Schutzgegenstand, S. 117 ff., 328 f.; Hoeren/Pinelli, JZ 2020, 879, 880 sowie Nachweis in Fn. 98.

116 Stieper in: Staudinger, $\mathbb{\$} 90$ BGB Rn. 17; Stresemann in: MüKo BGB, $\mathbb{9} 90$ Rn. 25; Berberich/Golla, PinG 2016, 165, 170; zweifelnd Hoeren, MMR 2019, 5, 5 ff.

117 Stieper in: Staudinger, $\$ 90$ BGB Rn. 13; BGH NJW 2016, 317 Rn. 19. 


\section{Deliktsrechtlicher Schutz von Daten}

Unabhängig von ihrer Sachqualität können Daten unter Umständen deliktsrechtlichen Schutz genießen. Denn insbesondere der Schutzbereich des Eigentums an einem Datenträger kann sich auf die darauf abgespeicherten Daten erstrecken. So erfolgt etwa die Löschung oder Änderung gespeicherter Daten im Wege physikalischer Veränderung des Datenträgers und kann so einen Eingriff in das an dem Speichermedium bestehende Eigentum darstellen. ${ }^{118}$ Allerdings versagt dieser über das Eigentum vermittelte Schutz für Daten in Konstellationen, in denen das Eigentum am Speichermedium und die etwaige „Berechtigung “119 an den Daten auseinander fallen. Ein praktisch bedeutsames Beispiel hierfür ist das Cloud-Computing. Hier steht das Eigentum an dem Server dem Internetdienstleister oder seinem Subunternehmer zu, während die Daten von den einzelnen Nutzern eingebracht werden. ${ }^{120}$

118 Zech, CR 2015, 137, 141 f.; Stieper in: Staudinger, $\$ 90$ BGB Rn. 19; Wagner in: MüKo BGB, $\mathbb{} 823$ Rn. 246, 332.

119 Mit dem hier verwendeten Begriff soll dem Ergebnis dieses Abschnitts, der gerade die Frage nach der Berechtigung an Daten zum Gegenstand hat, nicht vorgegriffen werden. Mit „Berechtigung“ soll lediglich zum Ausdruck kommen, dass beispielsweise der Autor oder der User, der die Speicherung vornimmt, eine Berechtigung an den Daten empfindet („meine Daten“), vgl. dazu bereits Fn. 36 und 37. Wenn die Speicherung aber nicht auf im eigenen Eigentum stehenden Speichermedien erfolgt (s. Cloud-Computing), ergeben sich ggf. (Delikts-)Schutzlücken.

120 Bock, AcP (217) 2017, 370, 380 konstatiert eine „immer stärker voranschreitende Entlokalisierung und Zersplitterung der Speicherung." Vgl. auch Boehm, ZEuP 2016, 358, 385; Bericht der Arbeitsgruppe „Digitaler Neustart“ der Konferenz der Justizministerinnen und Justizminister der Länder, S. 48 f., abrufbar a.a.O. (Fn. 43). Ähnliche Konsequenzen können sich im herkömmlichen Arbeitsverhältnis ergeben, wenn der Arbeitnehmer (und spätere Erblasser) sein berufliches E-Mail-Postfach auf einem privaten Endgerät nutzt bzw. wenn er vom Arbeitgeber zur Verfügung gestellte Endgeräte (auch) im privaten Kontext nutzt. Ferner ist an unter Eigentumsvorbehalt erworbene oder gemietete Hardware zu denken. 
a) Recht am Datenbestand als „Sonstiges Recht“ iSv. \823 Abs. 1 BGB

Den Schutzlücken, ${ }^{121}$ die aus der Entlokalisierung der Speicherung folgen, ${ }^{122}$ wollen einige Stimmen in der Literatur begegnen, indem sie ein Recht am Datenbestand als „sonstiges Recht“ im Rahmen von $\mathbb{8} 823$ Abs. 1 BGB annehmen: Der Schutzumfang des Deliktsrechts könne nicht davon abhängen, auf welche Weise Daten übermittelt und gespeichert werden. Ansonsten verfehle das Deliktsrecht seine Aufgabe, auch angesichts moderner technischer Phänomene Lösungen auf Grundlage der Wertungen des $₫ 823$ Abs. 1 BGB zu finden. In dem Maße, in dem mit Hilfe neuer Technologien Daten losgelöst vom Sacheigentum gespeichert werden, müsse das Privatrecht Schritt halten und den Schutz des „Dateneigentums" gewährleisten. ${ }^{123}$

Ausgangspunkt der Anerkennung eines Rechts als „sonstiges Recht“ im Rahmen von $₫ 823$ Abs. 1 BGB ist stets, dass es sich um ein eigentumsähnliches Recht handeln muss. So wie die in $\$ 823$ Abs. 1 BGB explizit genannten Rechte und Rechtsgüter muss auch jedes „sonstige Recht“ eine absolute, gegenüber jedermann wirkende Rechtsposition sein. ${ }^{124}$ Die ausschließliche Stellung muss gerade aus der Rechtsposition folgen und darf nicht lediglich - etwa technisch bedingt - faktischer Natur sein. ${ }^{125}$ Für das Recht am Datenbestand ist darauf hinzuweisen, dass $\$ 823$ Abs. 1 BGB ein bestehendes Recht oder Rechtsgut voraussetzt und dessen Zuweisung gerade nicht selbst vornimmt. ${ }^{126}$ Es darf somit nicht vorschnell von einem

121 Faust, Gutachten zum 71. DJT, 2016, S. A 77 f.; Stieper in: Staudinger, $\$ 90$ BGB Rn. 19; a.A. Hager in: Staudinger, $\mathbb{S} 823$ BGB Rn. B 192, der kein „unabdingbares Bedürfnis“ für eine weitere Ausdehnung jenseits des bereits geschützten Eigentums am Datenträger sieht. Instruktiv auch Herzog/Pruns, Der digitale Nachlass in der Vorsorge- und Erbrechtspraxis, S. $10 \mathrm{f}$.

122 Ebd. Fn. 120.

123 Wagner in: MüKo BGB, $\ 823$ Rn. 332.

124 Sprau in: Palandt, $\mathbb{8} 823$ BGB Rn. $11 ;$ A. Staudinger in: HK-BGB, $\mathbb{8} 823$ BGB Rn. 28; Förster in: BeckOK BGB, $\$ 823$ Rn. 143; ferner Berberich/Golla, PinG 2016, 165, $172 \mathrm{f}$.

125 BGH NJW 2012, 2034 Rn. 23 zu Domains. Das gilt sogar, wenn die faktische Position eigentumsfähig im Sinne von Art. 14 Abs. 1 S. 1 GG ist, vgl. a.a.O. Rn. 27 sowie Sprau in: Palandt, $\mathbb{\$} 823$ BGB Rn. 11. Zu Domains als Rechtsobjekt des digitalen Nachlasses vgl. bereits die Nachweise in Fn. 23. Siehe ferner Bericht der Arbeitsgruppe „Digitaler Neustart“ der Konferenz der Justizministerinnen und Justizminister der Länder, S. 46 f., abrufbar a.a.O. (Fn. 43).

126 Thiesen, Daten in der Erbmasse, S. 164; Determann, ZD 2018, 503, 504; Kübling/ Sackmann, ZD 2020, 24, 26 a.E. 
Schutzbedürfnis auf einen bestehenden Schutz de lege lata geschlossen werden. Das gilt insbesondere für das Recht an Daten, für welches die Debatte darüber, wem und auf welche Weise das Recht zuzuordnen wäre, noch in vollem Gange ist. ${ }^{127}$ Vor diesem Hintergrund wäre bei der unterstellten Aufnahme des Datenrechts als „sonstiges Recht“ die Frage nach der Aktivberechtigung noch offen, obschon bereits ein geschütztes Rechtsgut ausgemacht wäre. Bezeichnenderweise hat selbst der Gesetzgeber in $\$ 303$ a StGB - in Abgrenzung zu $\$ 303$ StGB - die Fremdheit der Daten gerade nicht zur Voraussetzung für die Strafbarkeit u.a. der Löschung oder Änderung der Daten gemacht. ${ }^{128}$ Zurecht weist Wagner insoweit darauf hin, dass die Komplexität der Frage kein Grund ist, mit der notwendigen Konkretisierungsarbeit gar nicht erst anzufangen. ${ }^{129}$ Gleichwohl stellt sich die Frage, ob derart zentrale Fragen wie die Schaffung und Zuordnung von Datenrechten - kurz eines umfassenden „Sachenrechts für Daten“130 nicht dem Gesetzgeber überlassen bleiben müssen. ${ }^{131}$

\section{b) Deliktsrechtlicher Schutz von Daten gem. $\$ 823$ Abs. 2 BGB}

Schon jetzt ist ein deliktsrechtlicher Schutz von Daten auch ohne die Anerkennung eines absoluten Rechts im zuvor dargestellten Sinne möglich. Gem. $\$ 823$ Abs. 2 BGB in Verbindung mit $\$ 303$ a StGB lösen indessen nur vorsätzliche Verletzungshandlungen eine Schadensersatzpflicht aus. Sofern mit Hinweis auf diese Beschränkung die Anerkennung eines absoluten

127 Faust, Gutachten zum 71. DJT, 2016, S. A 84 mit instruktiven Beispielen komplexer Zuordnungsfragen; das sieht so auch Wagner in: MüKo BGB, $\mathbb{8} 823$ Rn. 336; weitere Beispiele bei Bericht der Arbeitsgruppe „Digitaler Neustart“ der Konferenz der Justizministerinnen und Justizminister der Länder, S. 42 f., abrufbar a.a.O. (Fn. 43).

128 Faust, Gutachten zum 71. DJT, 2016, S. A 84.

129 Wagner in: MüKo BGB, $\mathbb{5} 823$ Rn.336f. m.w.N. zu entsprechenden Vorschlägen.

130 Faust, Gutachten zum 71. DJT, 2016, S. A 84; lediglich für einen Datenbesitz als „Ausschließlichkeitsrecht schwächerer Art“ wirbt Hoeren, MMR 2019, 5, 5 ff.

131 Boehm, ZEuP 2016, 358, 384 f. etwa warnt vor kollidierenden Rechtspositionen, wenn durch die Anerkennung eines eigenen Rechts am Datenbestand iSv. $\$ 823$ Abs. 1 BGB neben nicht personenbezogenen Daten auch personenbezogene Daten, die dann auch durch das Persönlichkeitsrecht geschützt seien, betroffen wären. Siehe aber Bericht der Arbeitsgruppe „Digitaler Neustart“ der Konferenz der Justizministerinnen und Justizminister der Länder, S. 51 f., abrufbar a.a.O. (Fn. 43). 
Rechts an Daten gefordert wird, um eine Haftung für fahrlässige Verletzungshandlungen gem. $₫ 823$ Abs. 1 BGB zu konstruieren, ${ }^{132}$ ist der folgende legislative Wille zu betonen. Der Gesetzgeber hat sich bewusst gegen eine deliktsrechtliche Generalklausel entschieden und die Ersatzfähigkeit reiner Vermögensschäden nur unter den restriktiven Voraussetzungen der \S 823 Abs. 2 und 826 BGB zugelassen. ${ }^{133}$ Eine Erweiterung der Haftung auch für fahrlässige Verletzungen eines Schutzgesetztes kann mithin richtigerweise ebenfalls nur der Gesetzgeber selbst vornehmen. ${ }^{134}$

\section{c) Rückschlüsse aus dem Deliktsrecht auf den digitalen Nachlass}

Es stellt sich die Frage, ob und inwiefern die soeben skizzierte Debatte für die Lösung der Probleme des digitalen Nachlasses fruchtbar gemacht werden kann. Das Deliktsrecht allgemein vermittelt den Schutz des Integritätsinteresses und $\$ 823$ Abs. 1 BGB speziell schützt die Integrität der dort genannten Rechtsgüter. ${ }^{135}$ Konkret bedeutet das für das Recht am Datenbestand insbesondere den Schutz vor Löschung und Veränderung der Daten. ${ }^{136}$ Die zentralen Fragen des digitalen Nachlasses sind hingegen im Kern solche der Verkehrsfähigkeit des Erbrechtsobjekts. Insofern die deliktsrechtliche Debatte dem Rechtsverkehr mit Daten den Weg bereitet, liegt darin auch ein Beitrag zur Lösung der Probleme rund um den digitalen Nachlass. Das gilt nicht zuletzt, weil die Rechtsnachfolge im Erbfall ein wichtiger Teilaspekt des Rechtsverkehrs mit Daten ist. Dieser Rechtsverkehr hat indes insbesondere die translative Übertragung von Ausschließlichkeitsrechten und den konstitutiven Erwerb von Tochterrechten

132 So ausdrücklich Wagner in: MüKo BGB, $\$ 823$ Rn. 338.

133 Teichmann in: Jauernig, BGB, Vor $\$ \$ 823$ ff. Rn. 2; Förster in: BeckOK BGB, $\$ 823$ Rn. 3; Faust, Gutachten zum 71. DJT, 2016, S. A 79 f.; A. Staudinger in: HK-BGB, Vorbemerkungen zu $\$ \$ \$ 823-853$ Rn. 8; Hager in: Staudinger, Vor $\$ \mathbb{S} 823 \mathrm{ff}$. BGB Rn. 19 f. spricht von „kleiner Generalklausel“.

134 Faust, Gutachten zum 71. DJT, 2016, S. A 86 f. schlägt eine entsprechende Regelung eines Schutzgesetztes im Rahmen des BSIG vor, um eine Haftung nach $\$ 823$ Abs. 2 BGB für fahrlässige Verletzungshandlungen zu ermöglichen. A.A. Wagner in: MüKo BGB, $\$ 823$ Rn. 339. Zur rechtspolitischen Debatte s. noch S. $66 \mathrm{f}$.

135 Vgl. nur Sprau in: Palandt, Einführung vor $₫ 823$ BGB Rn. 1, $\mathbb{8} 823$ BGB Rn. 2.

136 Zech, CR 2015, 137, 139 sowie grundlegend ders., Information als Schutzgegenstand, S. 115 ff.; Berberich/Golla, PinG 2016, 165, 170; ferner Determann, ZD 2018, 503, 504. 
zur Grundlage - und erst nachrangig den Integritätsschutz. ${ }^{137}$ Der Integritätsschutz alleine schließt nicht die Zuweisung aller Befugnisse an dem geschützten Recht ein: Die Übertragung eines Rechts oder der Zugang zu einem Gegenstand gehen über den rein abwehrrechtlichen Integritätsschutz hinaus. ${ }^{138}$ Auch das allgemeine Persönlichkeitsrecht aus Art. 2 Abs. 1 GG in Verbindung mit Art. 1 Abs. 1 GG ist deliktsrechtlich als Rahmenrecht in $\$ 823$ Abs. 1 BGB geschützt, aber weder uneingeschränkt übertragbar noch vollumfänglich vererblich. ${ }^{139}$ Sobald und soweit die fortdauernde Diskussion zum Deliktsrecht die bislang offenen Zuordnungsfragen des Datenrechts klären kann, lassen sich die sodann zugeordneten Datenrechte auch als Erbrechtsobjekte kategorisieren und einer Rechtsnachfolge im Erbfall zuführen. Da aber selbst diejenigen Stimmen, welche die Anerkennung eines Rechts am Datenbestand als „sonstiges Recht“ fordern, einräumen müssen, dass die Zuordnungsfrage (noch) offen ist, ${ }^{140}$ hilft die betreffende Debatte dem digitalen Nachlass (noch) nicht weiter.

Allerdings kann der durch $\mathbb{8} 823$ Abs. 1 BGB vermittelte Integritätsschutz vertragliche Ansprüche mitunter ergänzen, sofern das Recht am Datenbestand als „sonstiges Recht“ anerkannt würde: Wenn ein Provider - wie etwa ein Cloud-Anbieter - in seinen AGB (unwirksam) die Unvererbbarkeit der Account-Daten beziehungsweise des zugrundliegenden Vertragsverhältnisses bestimmt und (in der Annahme der Wirksamkeit seiner AGB) die gespeicherten Daten nach dem Tod des Erblassers löscht. ${ }^{141}$ In diesem Fall könnte neben die vertraglichen Ansprüche auch ein deliktischer Anspruch treten, der soweit möglich auf Wiederherstellung des Datensatzes, anderenfalls auf Wertersatz, gerichtet ist. ${ }^{142}$ Dies gilt freilich mit allen bekannten Schwächen der deliktischen Haftung im Vergleich zur

137 Zech, CR 2015, 137, 139 f.; vgl. auch Grützmacher, CR 2016, 485, 489 f.

138 Thiesen, Daten in der Erbmasse, S. 164; Zech, GRUR 2015, 1151, 1158; ders., CR 2015, 137, 140 und 143; Berberich/Golla, PinG 2016, 165, 170; Determann, ZD 2018, 503, 504.

139 Vgl. nur Hager in: Staudinger, $\mathbb{\$} 823$ BGB Rn. C 50b f. und C 34 ff.; zur Vererbbarkeit des allgemeinen Persönlichkeitsrechts s. S. $106 \mathrm{ff}$.

140 S. die Nachweise in Fn. 129. Die Gegenseite wiederum ist freilich noch strenger und macht die Probleme der Zuordnung zum zentralen Gegenargument gegen die Anerkennung des Rechts am Datenbestand, vgl. hierzu die Nachweise in Fn. 126, 127 und 128.

$141 \mathrm{Zu}$ AGB betreffend die Vererbbarkeit von Nutzerkonten vgl. noch auf S. $166 \mathrm{ff}$.

142 Stieper in: Staudinger, $\mathbb{9} 90$ BGB Rn. 19; Bericht der Arbeitsgruppe „Digitaler Neustart" der Konferenz der Justizministerinnen und Justizminister der Länder, S. 46, abrufbar a.a.O. (Fn. 43). 
vertraglichen Schadensersatzhaftung. ${ }^{143}$ Die praktische Relevanz dieser Konstellation dürfte zudem insofern abnehmen, als dass die vormals hohen Kosten, die mit der Speicherung der Daten verbunden waren, sinken, während der Wert der Nutzerdaten - möglichweise auch über den Tod des Nutzers hinaus - an Wert gewinnen. Das Interesse der Provider an einer zügigen Löschung nach dem Erbfall dürfte daher begrenzt sein. ${ }^{144}$

\section{d) Zwischenergebnis}

Für den Moment sei nach alledem festgehalten, dass selbst wenn man die Geltung eines umfassenden deliktsrechtlichen Integritätsschutzes des Datenbestands vor vorsätzlichen sowie fahrlässigen Verletzungshandlungen bejahte, daraus keine unmittelbaren Rückschlüsse auf Fragen nach der hier vorrangig relevanten Verkehrsfähigkeit des geschützten Rechts folgen. ${ }^{145}$ In demselben Maße, in dem der durch $₫ 823$ Abs. 1 BGB vermittelte Integritätsschutz zur Begründung des Rechtsverkehrs mit Daten zu kurz greift, greift auch die deliktsrechtliche Debatte als Beitrag zu Fragen des digitalen Nachlasses zu kurz.

\section{Urheberrecht}

Das Immaterialgüterrecht im Allgemeinen und das Urheberrecht im Besonderen könnten ebenfalls zur Begründung eines Rechts an Daten herangezogen werden. Funktional und begrifflich erscheint das auf den ersten Blick vielversprechend. Nachdem Daten - wie gesehen - mangels Körperlichkeit dem Sachenrecht unzugänglich sind, ${ }^{146}$ scheint das Immaterialgüterrecht prädestiniert, da es die rechtliche Zuordnung immaterieller Güter zur Aufgabe hat. Allerdings ist zu beachten, dass das Immaterialgüterrecht nur begrenzt analogiefähig ist. ${ }^{147}$ Ungeachtet der umstrittenen Frage, ob

143 Zum Vergleich zwischen Delikt und Vertrag s. nur Hager in: Staudinger, Eckpfeiler, T. Rn. 109 m.w.N.

144 Im Gegenteil wegen des Löschungsinteresses der Provider (noch) zur Eile ratend Bräutigam in: DAV-Stellungnahme Nr. 34/2013, S. $25 \mathrm{ff}$.

145 Ähnlich auch Thiesen, Daten in der Erbmasse, S. $159 \mathrm{ff}$.

146 Dazu bereits zuvor auf S. $48 \mathrm{f}$.

147 Pierson/Ahrens/Fischer, Recht des geistigen Eigentums, S. 71; Lettl, Gewerblicher Rechtsschutz, $\mathbb{S} 1$ Rn. 10 m.w.N.; Chrocziel, Einführung in den Gewerblichen Rechtsschutz und das Urheberrecht, Rn. $14 \mathrm{ff}$. 
das geistige Eigentum (sogar) einem strengen Numerus clausus unterliegt, ${ }^{148}$ begründet die folgende Überlegung die jedenfalls nur eingeschränkte Flexibilität des Rechtsgebiets. Die Rechtsordnung gewährt durch die Zuteilung eines Ausschließlichkeitsrechts ein Monopol - etwa im Falle des Patenrechts an einer Erfindung, im Falle des Markenrechts an einem Kennzeichen und im Falle des Urheberrechts an einer Schöpfung. ${ }^{149}$ Es obliegt primär dem Gesetzgeber, in der Abwägung zwischen der Freiheit von Informationen und sonstigen immateriellen Gütern einerseits und dem Schutz einer Investitions-, Schöpfungs- oder Erfindungsleistung andererseits zu entscheiden, ob und inwiefern ein (neues) Ausschließlichkeitsrecht gerechtfertigt ist. ${ }^{150}$

Ein eigenes, explizit geregeltes Immaterialgüterrecht an Daten als solchen besteht nicht. Vor dem soeben dargestellten Hintergrund müsste ein bestehendes Immaterialgüterrecht auf Daten als Schutzobjekt unmittelbar Anwendung finden. Dafür kommt allenfalls das Urheberrecht und dort insbesondere der Urheberrechtsschutz von Datenbankwerken gem. $\$ 4$ Abs. 2 UrhG sowie der Leistungsschutz von Datenbanken gem. $\mathbb{S} 887$ ff. UrhG in Betracht. ${ }^{151}$

148 Ausführlich dazu Ohly in: FS Schricker, S. 105 ff. m.w.N. sowie Schroeder, Numerus clausus der Immaterialgüterrechte?, in: Specht/Lauber-Rönsberg/Becker (Hrsg.), Medienrecht im Medienumbruch, Baden-Baden 2017, S. 9 ff. m.w.N.

149 Instruktiv und differenzierend dazu Bisges in: Bisges (Hrsg.), Handbuch Urheberrecht, Kap. 1 Rn. 18 ff.; Schack, Urheber- und Urhebervertragsrecht, Rn. 14 f., 69.

150 Bejahend etwa Kleespies, GRUR 2002, 764, 767; Berberich, WRP 2011, 543, 547; ders., Virtuelles Eigentum, S. $216 \mathrm{ff}$; differenzierend Obly in: FS Schricker, S. 121 der vom „Grundsatz strikter Subsidiarität ergänzender Schutzformen“ spricht. Ungeachtet der genauen Formulierung lehnt auch Obly einen sui-generis Schutz von Daten als solchen ab, vgl. a.a.O., S. 105, dort in Fn. 1.

151 Siehe dazu Specht, CR 2016, 288, 293 f.; Dorner, CR 2014, 617, 621 f.; Grützmacher, CR 2016, 485, 487 f.; Wandtke, MMR 2017, 6, 11 f.; Wiebe, GRUR 2017, 338, 338 ff.; Ensthaler, NJW 2016, 3473, 3474 f.; Thiesen, Daten in der Erbmasse, S. 149 ff.; speziell zu Accounts vgl. Kutscher, Der digitale Nachlass, S. 29 ff.; Bericht der Arbeitsgruppe „Digitaler Neustart“ der Konferenz der Justizministerinnen und Justizminister der Länder, S. 57 f., abrufbar a.a.O. (Fn. 43). 
a) Urheberrechtsschutz an Sammel- und Datenbankwerken gem. $\$ 4$ UrhG

Datenbankwerke sind nach $\$ 4$ Abs. 2 UrhG solche Sammelwerke (vgl. $\$ 4$ Abs. 1 UrhG), deren Elemente systematisch oder methodisch angeordnet und einzeln mit Hilfe elektronischer Mittel oder auf andere Weise zugänglich sind. ${ }^{152}$ Bei Datenbank- und Sammelwerken weist die Auswahl- und Anordnungsentscheidung einen solchen Grad an Individualität auf, dass sie als persönliche geistige Schöpfung urheberrechtlich schutzfähig werden. ${ }^{153}$ An dieser Hürde scheitern sowohl die schlichte Aneinanderreihung von Daten als auch die auf Vollständigkeit angelegte Datenbank, da in keinem der beiden Fällen eine schöpferische Entscheidung zu fällen ist. ${ }^{154}$ Ferner scheitert der Schutz ausschließlich automatisch erhobener Datenbanken mangels menschlicher Leistung am Merkmal "persönlich“. ${ }^{155}$ Schon allein vor diesem Hintergrund erscheint es fraglich, ob sich durch $\$ 4$ UrhG ein geistiges Eigentum an Daten begründen lässt. ${ }^{156}$ Denn die Idee des „Dateneigentums“ beruht darauf, ein umfassendes Zuordnungsregime zu begründen. Mit dem Merkmal der persönlichen geistigen Schöpfung wird aber gerade in schützenswerte Datenmengen und andere Datenmengen unterschieden. Diese Prüfung erfolgt anhand des urheberechtlichen Wertungsmaßstabs, der anders als etwa das Sachenrecht nicht jedem Gegenstand ohne Ansehung seines (schöpferischen) Wertes Schutz zukommen lassen soll. In Ermangelung einer eigenen Regelung wird das Datenrecht durch die behelfsmäßige Anwendung des Urheberrechts dann mit derselben restriktiven Wertung konfrontiert. Ob und inwieweit einem Datensatz Schutz zuteilwird, kann aus urheberrechtlicher Sicht zwar eine ausgewogene Entscheidung darstellen, aus Sicht der Daten-Governance aber zufällig anmuten.

152 Leistner in: Schricker/Loewenheim (Hrsg.), $\$ 4$ UrhG Rn. 49 ff.; Schack, Urheberund Urhebervertragsrecht, Rn. $154 \mathrm{f}$; A Alberg in: BeckOK Urheberrecht, $\mathbb{} 4$ UrhG Rn. 18 ff.; Marquardt in: Wandtke/Bullinger (Hrsg.), UrhG, $\$ 4$ Rn. 8.

153 Leistner in: Schricker/Loewenheim (Hrsg.), $\$ 4$ UrhG Rn. 1.; Marquardt in: Wandtke/Bullinger (Hrsg.), UrhG, $\$ 4$ Rn. 9 m.w.N.; Specht, CR 2016, 288, 293; Wandtke, MMR 2017, 6, 11.

154 Schack, Urheber- und Urhebervertragsrecht, Rn. 155 f.; Bisges in: Bisges (Hrsg.), Handbuch Urheberrecht, Kap. 1 Rn. 143, 146; Dreier in: Dreier/Schulze (Hrsg.), UrhG, $₫ 4$ Rn. 20.

155 Specht, CR 2016, 288, 293; Dorner, CR 2014, 617, 621.

156 Zweifelnd auch Specht, CR 2016, 288, 293; Dorner, CR 2014, 617, 621; Bericht der Arbeitsgruppe „Digitaler Neustart“ der Konferenz der Justizministerinnen und Justizminister der Länder, S. 57, abrufbar a.a.O. (Fn. 43). 
Darüber hinaus ist, auch bei unterstelltem Werkschutz nach $₫ 4$ UrhG, der Blick auf den Schutzumfang zu lenken. Nach dem Gesetzeswortlaut müssen die einzelnen Elemente des Datenbankwerks ihrerseits nicht zwingend Werkcharakter aufweisen. ${ }^{157}$ Umgekehrt werden die einzelnen Elemente aber auch nicht selbst zum Schutzobjekt. ${ }^{158}$ So ist ein Datenbankwerk gegen die Übernahme der Struktur oder aller bzw. zumindest vieler Elemente geschützt. ${ }^{159}$ Dagegen berührt der Zugriff auf einzelne Daten den Schutzbereich nach $₫ 4$ Abs. 2 UrhG gerade nicht. ${ }^{160}$

Aus $\mathbb{} 4$ Abs. 1 und 2 UrhG lässt sich nach alledem kein geeignetes $\mathrm{Zu}$ ordnungsregime für Daten ableiten. Zwar bleibt der Schutz einzelner Datenbankwerke unbenommen mit der Konsequenz, dass die jeweiligen Datensätze mitunter reflexhaft zum Gegenstand eines Ausschließlichkeitsrechts werden. Gleichwohl werden diese Fälle aber nicht ausreichend praktische Relevanz aufweisen, um die Diskussion zum „Dateneigentum“ entscheidend zu beeinflussen. ${ }^{161}$

\section{b) Leistungsschutz des Datenbankherstellers gem. $\$ \$ 87$ a ff. UrhG}

Datenbanken können auch unter dem Aspekt des Investitionsschutzes durch das UrhG geschützt sein. Die Legaldefinition der Datenbank nach $\$ 87$ a Abs. 1 S. 1 UrhG unterscheidet sich von der des Datenbankwerks gem. $\$ 4$ Abs. 2 UrhG vor allem dadurch, dass statt einer persönlichen geis-

$157 \rrbracket 4$ Abs. 1 UrhG („[...] unbeschadet eines an den einzelnen Elementen gegebenenfalls bestehenden Urheberrechts oder verwandten Schutzrechts [...]" Hervorhebung nicht im Original) gilt insoweit auch für $\$ 4$ Abs. 2 UrhG; vgl. hierzu Dreier in: Dreier/Schulze (Hrsg.), UrhG, $\$ 4$ Rn. 4; Ablberg in: BeckOK Urheberrecht, $\$ 4$ UrhG Rn. 33; Wiebe in: Spindler/Schuster (Hrsg.), Recht der elektronischen Medien, $\mathbb{} 4$ UrhG Rn. 5; ausführlich zu den Elementen eines Sammelbzw. Datenbankwerks Czychowski in: Fromm/Nordemann, $\mathbb{} 4$ UrhG Rn. $16 \mathrm{ff}$.

158 Dreier in: Dreier/Schulze (Hrsg.), UrhG, $\$ 4$ Rn. 4; Kotthoff in: Dreyer/Kotthoff/ Meckel/Hentsch (Hrsg.), Urheberrecht, $\$ 4$ UrhG Rn. 21.

159 Wiebe in: Spindler/Schuster (Hrsg.), Recht der elektronischen Medien, $\mathbb{} 4$ UrhG Rn. 17; Kotthoff in: Dreyer/Kotthoff/Meckel/Hentsch (Hrsg.), Urheberrecht, $\$ 4$ UrhG Rn. 21.

160 Leistner in: Schricker/Loewenheim (Hrsg.), $\$ 4$ UrhG Rn. 34; Wiebe in: Spindler/ Schuster (Hrsg.), Recht der elektronischen Medien, \4 UrhG Rn. 2; Czychowski in: Fromm/Nordemann, $\mathbb{} 4$ UrhG Rn. 40 m.w.N.; Specht, CR 2016, 288, 293; Determann, ZD 2018, 503, 505.

161 Explizit mit Hinweis auf mangelnde praktische Relevanz auch Zieger/Smirra, MMR 2013, 418, 419 und Dorner, CR 2014, 617, 621. 
tigen Schöpfung eine nach Art oder Umfang wesentliche Investition in den Inhalt der Datenbank vorausgesetzt ist. ${ }^{162}$ Die $\$ \$ 87$ a ff. UrhG gewähren ein „Recht der Entnahme“ 163 und schützen damit den Rechtsinhaber davor, dass quantitativ (Datenvolumen) oder qualitativ (Investitionsvolumen) wesentliche Teile der Datenbank von Dritten übernommen werden. ${ }^{164}$ Hingegen folgt aus dem Datenbankschutz kein Schutz einzelner Elemente der Datenbank oder gar ein selbstständiges, abtrennbares Recht an den jeweiligen Elementen. ${ }^{165}$ Erfasst ist also insbesondere nicht die Möglichkeit, über einzelne Daten zu verfügen oder sie gegen Vervielfältigung oder Kenntnisnahme zu schützen. Auch das Leistungsrecht des Datenbankherstellers vermag daher nicht, einem Recht an Daten den Weg zu bereiten. ${ }^{166}$

c) Bezug zum digitalen Nachlass: Rechtsinhaberschaft und Reichweite des Rechts

Es fragt sich, welche Konsequenzen ein urheberrechtlich grundiertes Recht an Daten - seine Geltung ungeachtet der soeben dargestellten Bedenken unterstellt - für den digitalen Nachlass hätte. Die Antwort hierauf hängt primär davon ab, wem das Recht in diesem Fall zustünde.

Wenn das Datenrecht zur Vereinfachung der Rechtsnachfolge als Erbrechtsobjekt dienen soll, müsste der Erblasser daran berechtigt sein. Das

162 Wandtke, MMR 2017, 6, 11 f.; ausführlich und differenzierend Dreier in: Dreier/ Schulze (Hrsg.), UrhG, $\mathbb{5} 87 \mathrm{a}$ Rn. 3 ff.; Schack, Urheber- und Urhebervertragsrecht, Rn. 291.

163 Vgl. Vogel in: Schricker/Loewenheim (Hrsg.), $\$ 87 b$ UrhG Rn. 37 zur europarechtskonformen Auslegung der Begriffe „Vervielfältigung“ und „Entnahme“; dazu auch Wiebe in: Spindler/Schuster (Hrsg.), Recht der elektronischen Medien, $\$ 87 b$ UrhG Rn. 2 ff.

164 Zech, GRUR 2015, 1151, 1158; Hermes in: Wandtke/Bullinger (Hrsg.), UrhG, \87b Rn. $11 \mathrm{ff}$; Wiebe in: Spindler/Schuster (Hrsg.), Recht der elektronischen Medien, $\$ 87 \mathrm{~b}$ UrhG Rn. $17 \mathrm{f}$.

165 Specht, CR 2016, 288, 293 f.; Dorner, CR 2014, 617, 622; Wandtke, MMR 2017, 6, 11; Thiesen, Daten in der Erbmasse, S. 151; ausführlich dazu Zech, Information als Schutzgegenstand, S. 360 f.; Determann, ZD 2018, 503, 505; Ensthaler, NJW 2016, 3473, $3474 \mathrm{f}$.

166 Nach Dorner, CR 2014, 617, 622 kommt im Gegenteil sowohl in $\$ 4$ UrhG als auch in $\$ 87$ a UrhG der „klare gesetzgeberische Wille zum Ausdruck, dass Informationen (als Ergebnisse einer Datenaggregation) frei von exklusiven rechtlichen Zuordnungen bleiben sollen.“; s. auch Ensthaler, NJW 2016, 3473, 3475. 
ist der Fall, wenn der Erblasser originärer Rechtsinhaber oder Lizenznehmer ist. Grundsätzlich kann das nur in Ansehung der konkreten Nutzungshandlung des Erblassers etwa im Rahmen eines bestimmten Internetdienstes beurteilt werden. Trotzdem lassen sich einige abstrakte Überlegungen formulieren. So wird in der Praxis ein Lizenzvertrag zwischen dem Erblasser als Lizenznehmer und einem Datenbank(werk)rechtsinhaber die seltene Ausnahme bilden. Mithin könnte der Erblasser als Nutzer von Internetdiensten allenfalls als originärer Rechtsinhaber in Betracht kommen. Dafür müsste er im Falle des $\$ 4$ UrhG die schöpferische Auswahl- oder Anordnungsentscheidung getroffen oder im Falle der $\$ \$ \$ 87 \mathrm{aff}$. UrhG die Investitionsleistung erbracht haben, vgl. $\$ 87$ a Abs. 2 UrhG.

Auch soweit bei der Nutzung von Internetdiensten systematisch Daten betreffend einen Nutzer erfasst und in einer Datenbank gespeichert und aufbereitet werden, wird die dafür erforderliche Leistung in der Regel durch den Provider erbracht. Dass die Daten die Person des Nutzers betreffen mögen, ist lediglich eine datenschutzrechtliche und nicht eine urheberrechtlich relevante Kategorie. Der Provider entwirft und unterhält eine technische Infrastruktur, die der Erblasser lediglich vorfindet und den Vorgaben entsprechend nutzt. So trifft der Nutzer nur in den seltensten Fällen eine schöpferische Auswahlentscheidung und wohl in keinem Fall tätigt er eine Investition. Sofern und soweit hier Daten(bank)rechte entstehen, stehen sie somit originär dem Provider zu. Es käme folglich nicht zu einer Vereinheitlichung des Erbrechtsobjekts durch ein Datenrecht, wenn dieses aus dem Urheberrecht folgte. Mithin lässt sich der urheberrechtliche Teilbereich der Diskussion um ein „Dateneigentum“ - auch wenn der Ansatz noch weiterentwickelt werden sollte - für den digitalen Nachlass kaum fruchtbar machen.

\section{Datenschutzrecht}

Auch das Datenschutzrecht könnte als Quelle eines Datenrechts in Betracht kommen. Allerdings ist die Funktion des Datenschutzes auch und gerade in der Ausprägung durch die DS-GVO nicht die Güterzuordnung. Vielmehr reguliert das Datenschutzrecht den Umgang mit personenbezogenen Daten und wäre folglich eher Schranke eines etwaigen Datenrechts. ${ }^{167}$ Insofern ist auf die zentrale Norm des Art. 6 DS-GVO hinzuweisen, der ein

167 Zech, CR 2015, 137, 141; Paal/Hennemann, NJW 2017, 1697, 1698; differenzierend Kübling/Sackmann, ZD 2020, 24, 27 ff. 
präventives Verbot mit Erlaubnisvorbehalt betreffend die Verarbeitung von personenbezogenen Daten statuiert. ${ }^{168}$

Ausweislich des Art. 6 Abs. 1 lit. f) DS-GVO ist das Datenschutzrecht ein abwägungsoffenes Recht und ähnelt damit dem Vorbild anderer Persönlichkeitsrechte. ${ }^{169}$ Schon im sog. Volkszählungsurteil des BVerfG ${ }^{170}$ sowie auch in der daran anknüpfenden Rechtsprechung das BGH, beispielsweise im Spickmich.de-Urteil, ${ }^{171}$ wird betont, dass eine absolute Herrschaft über die „eigenen Daten“ mit dem Leben in der sozialen Gemeinschaft kollidiere. ${ }^{172}$ Zur Wahrnehmung der Informations- und Meinungsfreiheit, müssen personenbezogene Daten (unter gewissen Umständen) auch ohne oder gegen den Willen des Betroffenen genutzt werden können. ${ }^{173}$ Das geltende Datenschutzrecht beabsichtigt, diese ohnehin schon komplexen Interessenkonflikte zu befrieden. ${ }^{174}$ Darüber hinaus auch die Zuweisung von Datenrechten in dieser Materie zu verorten, würde eine Kommerzialisierung des Datenschutzes bedeuten und wäre jedenfalls im geltenden Datenschutzrecht systemfremd. ${ }^{175}$ Das gilt umso mehr vor dem Hintergrund, dass die DS-GVO auf nicht-personenbezogene Daten gar keine Anwendung findet, vgl. Art. 2 Abs. 1 DS-GVO, und schon deshalb nicht Anknüpfungspunkt für ein hieraus abgeleitetes umfassendes „Dateneigentum“ sein kann. ${ }^{176}$ Insofern ist Zech zuzustimmen, wenn er zusammenfassend feststellt, dass sich aus dem Datenschutzrecht allenfalls ein rahmenrechtliches

168 Frenzel in: Paal/Pauly (Hrsg.), DS-GVO, Art. 6 Rn. 1 f.; Albrecht in: NK-Datenschutzrecht, Einführung zu Art. 6 DS-GVO Rn. 1 f.; Kühling/Klar/Sackmann, Datenschutzrecht, Rn. 323; kritisch dazu Albers/Veit in: BeckOK Datenschutzrecht, Art. 6 DS-GVO Rn. 2.

169 Zech, GRUR 2015, 1151, 1154. Ausführlich zur Abwägung am Maßstab von Art. 6 Abs. 1 lit. f) DS-GVO vgl. auf S. 130 ff.

170 BVerfGE 65, 1, 43 f. = BVerfG NJW 1984, 419, 422.

171 BGHZ 181, 328, 338 = BGH NJW 2009, 2888, 2892.

172 Taeger, Datenschutzrecht. Einführung, S. 36 ff., 38; Zech, GRUR 2015, 1151, 1154; Specht, GRUR Int. 2017, 1040, $1041 \mathrm{f}$.

173 Härting, CR 2016, 646, 648; ders., Internetrecht, Kap. A Rn. 83 ff.; Thiesen, Daten in der Erbmasse, S. 147.

174 Albrecht/Jotzo, Das neue Datenschutzrecht der EU, S.74f. mit Hinweis auf die Erwägungsgründe 47-49 DS-GVO; Kühling/Klar/Sackmann, Datenschutzrecht, Rn. 399; in diesem Sinne, aber zur alten Rechtslage vor Geltung der DS-GVO Taeger, Datenschutzrecht. Einführung, S. $36 \mathrm{ff}$.

175 Specht, GRUR Int. 2017, 1040, 1041 f.; Dorner, CR 2014, 617, 623 ff.

176 Paal/Hennemann, NJW 2017, 1697, 1698 m.w.N.; Specht, GRUR Int. 2017, 1040, 1042; Kübling/Sackmann, ZD 2020, 24, 29; entsprechend bewertet Härting, CR 2016, 646, 648 den Personenbezug als Anknüpfungspunkt für ein Datenrecht auch als ungeeignet. 
„Recht an den eigenen persönlichen Daten“ ergibt, hingegen aber kein eigentumsartiges „Recht am eigenen Datum“. ${ }^{177}$

Der datenschutzrechtliche Teil der Debatte zum „Dateneigentum“ und der digitale Nachlass kommen einander am nächsten bei der Frage, ob und inwieweit die subjektiven Rechte, die durch das Datenschutzrecht gewährt werden, vererbbar sind. ${ }^{178}$ Diese Rechte folgen aus Kapitel III der DSGVO, das die Überschrift „Rechte der betroffenen Person“, trägt, vgl. Art. 12 ff. DS-GVO. Das Ob und das Wie der Vererbbarkeit hängen (auch) davon ab, wie eng datenschutzrechtliche Befugnisse an die Persönlichkeit des Betroffenen gebunden sind oder ob eine gewisse Selbständigkeit und damit letztlich Kommerzialisierung der Befugnisse möglich ist. Die Argumente zum datenschutzrechtlichen „Dateneigentum“ können also auch für die konkrete Frage der Vererbbarkeit fruchtbar gemacht werden. ${ }^{179}$

\section{5. (Lauterkeitsrechtlicher) Leistungs- und Geheimnisschutz}

Auch das Lauterkeitsrecht kommt für die Konstruktion eines Datenrechts in Betracht. Insoweit ließe sich zum einen an den lauterkeitsrechtlichen Leistungsschutz, ${ }^{180} \mathbb{\$} 3$ Abs. 1, 4 Nr. 3 UWG, denken und zum anderen an den Geheimnisschutz, ${ }^{181}$ der bislang vor allem in den $\mathbb{S} 17 \mathrm{ff}$. UWG a.F. geregelt war und sich nunmehr im Gesetz zum Schutz von Geschäftsgeheimnissen (GeschGehG) findet.

177 Zech, GRUR 2015, 1151, 1155.

178 Dazu Solmecke/Köbrich/Schmitt, MMR 2015, 291, 292 f.; LG Berlin ZEV 2016, 189, 194; dagegen aber KG Berlin ZEV 2017, 386 Rn. 104 jeweils zur Rechtslage vor Geltung der DS-GVO.

179 Zur Eröffnung des Anwendungsbereichs der DS-GVO im Kontext des digitalen Nachlasses s. S. $119 \mathrm{ff}$.

180 Dazu Becker, GRUR 2017, 346, passim; noch zur alten Rechtslage unter $₫ 4$ Nr. 9 UWG a.F. Zech, Information als Schutzgegenstand, S. $364 \mathrm{f}$.

181 Zur Rechtslage unter den $\mathbb{S} \mathbb{S} 17 \mathrm{ff}$. UWG a.F. vgl. Zech, GRUR 2015, 1151, 1155 f.; Grützmacher, CR 2016, 485, 488 f.; Dorner, CR 2014, 617, 622 f.; Thiesen, Daten in der Erbmasse, S. 147 ff. Das neue GeschGehG ändert an den grundlegenden Argumenten, nach denen aus dem Geheimnisschutz nach Maßgabe der $\mathbb{S} \mathbb{S} 17 \mathrm{ff}$. UWG a.F. kein Recht an Daten abgeleitet werden kann, wenig. 
a) Lauterkeitsrechtlicher Leistungsschutz, $\mathbb{\$} \$ 3$ Abs. 1, 4 Nr. 3 UWG

Der lauterkeitsrechtliche Leistungsschutz erlaubt keine präzise Nutzungszuweisung, sondern verbietet lediglich die (allzu) direkte - unlautere Ausnutzung fremder Leistungen. ${ }^{182}$ Eine solche Leistung kann zwar auch in Form von Daten bestehen; die Daten sind im Falle von $\$ 4 \mathrm{Nr} .3$ lit. a), b) UWG dann selbst die Produkte oder Teil eines Angebots sowie im Falle von $\$ 4$ Nr. 3 lit. c) UWG für die Herstellung anderer Produkte oder Angebote erforderlich. ${ }^{183} \mathrm{Da}$ die Kriterien des UWG-Leistungsschutzes vor allem zu physischen, sichtbaren Produkten entwickelt wurden und deshalb an optischen Merkmalen orientiert sind, passt $\$ 4$ Nr. 3 UWG aber nur eingeschränkt auf Daten. ${ }^{184}$ Schwerer wiegt noch die Überlegung, dass die Orientierung und Weiterentwicklung an auf dem Markt bereits vorhandenen Produkten grundsätzlich ein zulässiger Teil des Wettbewerbs ist. Außerhalb von Sondergesetzen wie dem PatG und UrhG wird Leistungsschutz durch die Generalklausel des $\$ 4 \mathrm{Nr} .3 \mathrm{UWG}$ nur vor unlauterer Herkunftstäuschung, Rufausbeutung und Industriespionage gewährt. ${ }^{185}$ Ein allgemeines Ausschließlichkeitsrecht am Datenbestand, welches einem Recht an Daten den Weg bereiten könnte, ist damit de lege lata unvereinbar, da es der gesetzgeberischen Prärogative zur Anerkennung neuer Leistungsschutzrechte vorgreifen würde.

\section{b) Geheimnisschutz, $\mathbb{S} \mathbb{S} 1 \mathrm{ff}$. GeschGehG}

Die Vorschriften des Nebenstrafrechts zum Schutz von Betriebs- und Geschäftsgeheimnissen in den $\$ \$$ 17-19 UWG a.F. wurden aufgehoben. An deren Stelle tritt das GeschGehG, welches unter der nunmehr einheitlichen Bezeichnung Geschäftsgeheimnisse im Sinne der Legaldefinition gem. $\$ 2$ Nr. 1 GeschGehG schützt. Mit der Normierung in einem Spezial-

182 Becker, GRUR 2017, 346, 346.

183 Becker, GRUR 2017, 346, 347 ff. spricht von Daten als Erzeugnissen sowie von Daten in Erzeugnissen; dem folgend Köhler in: Köhler/Bornkamm/Feddersen (Hrsg.), $\$ 4$ UWG Rn. 3.22.

184 Becker, GRUR 2017, 346, $347 \mathrm{f}$.

185 Emmerich/Lange, Unlauterer Wettbewerb, S.131f. m.w.N. Auch die Rspr. nimmt zugunsten der Wettbewerbsfreiheit den Grundsatz der Nachahmungsfreiheit an, vgl. BGH GRUR 2007, 795 Rn. 51; BGH GRUR 2008, 1115 Rn. 32; strenger aber etwa Köhler in: Köhler/Bornkamm/Feddersen (Hrsg.), $\$ 4$ UWG Rn.3.3. 
gesetz wurde der Schutz von Geschäftsgeheimnissen von seinem Status als Annexmaterie des UWG befreit. ${ }^{186}$ Das nunmehr eigene Rechtsgebiet behält zwar die Berührungspunkte zum Lauterkeitsrecht, weist aber in höherem Maße als bislang Wesenszüge des geistigen Eigentums auf. ${ }^{187}$ Doch auch unter der Geltung des GeschGehG bleibt es dabei, dass der Geheimnisschutz keine eigenständige rechtliche Zuweisung bewirkt, sondern lediglich eine faktische Ausschließlichkeit verstärkt. ${ }^{188}$ Zwar gewähren die \$S 6 ff. GeschGehG dem Rechtsinhaber eine Reihe von Ansprüchen im Falle einer Verletzung des Geheimnisschutzes. Der Schutz besteht aber neben anderen Voraussetzungen - überhaupt nur dann, wenn die infrage stehende Information nicht allgemein bekannt oder ohne Weiteres zugänglich ist und außerdem den Gegenstand von angemessenen Geheimhaltungsmaßnahmen durch ihren Inhaber bildet, vgl. $\$ 2 \mathrm{Nr} .1$ lit. a) und b) GeschGehG. ${ }^{189}$ Gibt der Inhaber die Information gegenüber einem Personenkreis preis, der nicht zur Vertraulichkeit verpflichtet ist, scheidet die Information folglich aus dem Schutzbereich des GeschGehG aus. Dass die Information dem Inhaber nicht absolut zugewiesen ist, zeigt sich auch daran, dass es Dritten unbenommen bleibt, sie durch eine eigenhändige Entdeckung bzw. Schöpfung oder (neuerdings) auch im Wege des Reverse Engineerings zu erlangen, vgl. $\$ 3$ Abs. 1 Nr. 1 und 2 GeschGehG. ${ }^{190}$

186 Vgl. das Vorwort in: Harte-Bavendamm/Ohly/Kalbfus (Hrsg.), GeschGehG (Geheimnisschutz lange Zeit als „Stiefkind Aschenputtel“).

187 Obly, GRUR 2019, 441, 450; Pierson/Abrens/Fischer, Recht des geistigen Eigentums, S. 47 f.; Harte-Bavendamm in: Harte-Bavendamm/Ohly/Kalbfus (Hrsg.), GeschGehG, Einl. A Rn. 127, 129; Berberich/Golla, PinG 2016, 165, 174; einschränkend aber Köhler in: Köhler/Bornkamm/Feddersen (Hrsg.), Vor $\mathbb{\$} \mathbb{1 7 - 1 9}$ UWG Rn. 12.

188 Pierson/Abrens/Fischer, Recht des geistigen Eigentums, S. 47 f.; Kalbfus in: HarteBavendamm/Ohly/Kalbfus (Hrsg.), GeschGehG, Einl. A Rn. 185 zur Abgrenzung vom Ausschließlichkeitsrecht an Patenten; wie hier zum alten Recht bereits Zech, GRUR 2015, 1151, 1156.

$189 \mathrm{Zu}$ den Schutzvoraussetzungen nach neuem Recht s. Ohly, GRUR 2019, 441, 442 ff. und Dann/Markgraf, NJW 2019, 1774, 1774 ff. sowie Harte-Bavendamm in: Harte-Bavendamm/Ohly/Kalbfus (Hrsg.), GeschGehG, $\$ 2$ Rn. 14 ff.; nach den Gesetzesmaterialien trägt der Geheimnisinhaber die Beweislast für die Etablierung angemessener Geheimhaltungsmaßnahmen, vgl. BT-Drs. 19/4724, S. 24.

190 Dann/Markgraf, NJW 2019, 1774, 1776 bezeichnen $\$ 3$ Abs. 1 Nr. 2 GeschGehG als einen Paradigmenwechsel im Geheimnisschutzrecht; dazu Obly in: Harte-Bavendamm/Ohly/Kalbfus (Hrsg.), GeschGehG, $\mathbb{} 3$ Rn. 19 ff. 
c) Lauterkeitsrecht und digitaler Nachlass

Wiederum stellt sich die Frage, ob die Debatte der Lösung des digitalen Nachlasses zuträglich sein kann. Sowohl der lauterkeitsrechtliche Leistungsschutz als auch der Geschäftsgeheimnisschutz begegnen hier einem ähnlich gelagerten Problem. Um den Erbgang im Kontext des digitalen Nachlasses zu vereinfachen, müsste auch und gerade der Datenbestand des Erblassers in dessen Dimension als Nutzer und Verbraucher erfasst und gegebenenfalls durch ein „Dateneigentum“ vereinheitlicht werden. Das Lauterkeitsrecht als Teil des Wettbewerbsrechts und der Schutz von $\mathrm{Ge}^{-}$ schäftsgeheimnissen zielen aber auf die Regulierung und den Schutz gewerblicher Handlungen. Für den Leistungsschutz folgt das bereits aus dem Wortlaut, der bestimmt, dass die Verletzungshandlung gem. $\$ 3$ Abs. 1 UWG eine „geschäftliche Handlung“ im Sinne des $\$ 2$ Abs. 1 Nr. 1 UWG sein muss und dass die nachgeahmten Waren oder Dienstleistungen gem. $\$ 4$ Nr. 3 UWG solche eines „Mitbewerbers“ im Sinne des $\$ 2$ Abs. 1 Nr. 3 UWG sein müssen. ${ }^{191} \mathrm{Im}$ Falle des GeschGehG ist ebenfalls eine weitgehende Beschränkung auf Geschäftsgeheimnisse unter Ausschluss von Privatgeheimnissen angebracht. Das folgt aus dem systematischen Zusammenhang und Erwägungsgrund 14 GeschGeh-RL ${ }^{192}$, in dem sich der Begriffsdefinition von "Geschäftsgeheimnissen“ ausschließlich über Beispiele aus dem gewerblichen Wettbewerbskontext angenähert wird. ${ }^{193}$

Im Ergebnis ist also festzuhalten, dass de lege lata weder aus dem Lauterkeitsrecht noch aus dem Geschäftsgeheimnisschutz ein „Dateneigentum“ abzuleiten ist. Aber selbst, wenn die oben beschriebenen Ansätze durch die Wissenschaft oder auch durch den Gesetzgeber in Richtung eines Rechts an Daten weiterentwickelt würden, so folgte daraus wohl keine Hilfestellung für den digitalen Nachlass. Dessen bedeutende private und persönlichkeitsrechtssensible Dimension wird durch das Wettbewerbsrecht kaum erfasst.

191 Köhler in: Köhler/Bornkamm/Feddersen (Hrsg.), \$4 UWG Rn. $3.18 \mathrm{f}$.

192 Richtlinie (EU) 2016/943 des Europäischen Parlaments und des Rates vom 8. Juni 2016 über den Schutz vertraulichen Know-hows und vertraulicher Geschäftsinformationen vor rechtswidrigem Erwerb sowie rechtswidriger Nutzung und Offenlegung, (ABl. Nr. L 157 S. 1), Celex-Nr. 32016 L 0943.

193 So auch Obly, GRUR 2019, 441, 442, der aber auf Ausnahmefälle hinweist, in denen es Überschneidungen geben könne, wie bei der Vermarktung eigener Persönlichkeitsaspekte durch Prominente oder bei Privatinformationen mit Einfluss auf den Wettbewerb - etwa Gesundheitsdaten leitender Angestellter. 
6. Ergebnis: Kein Recht an Daten de lege lata

Nach alledem gilt, dass es de lege lata in Deutschland kein eigentumsähnliches Recht an Daten gibt. Zwar kann angesichts zahlreicher gesetzlicher Regelungen, die (auch) auf Daten oder auf durch sie verkörperte Informationen Anwendung finden, nicht von einer völlig unregulierten Materie ausgegangen werden. Mitunter fallen Datenbestände auch in den Anwendungsbereich eines Regimes, das absolute Rechte an den Daten oder den verkörperten Inhalten gewährt. Als Beispiel sei hier der urheberrechtliche Schutz von Software nach Maßgabe der $\$ \$ \$ 69 \mathrm{aff}$. UrhG genannt. In keinem dieser Fälle liegt die Begründung für den Schutz jedoch in der Eigenschaft der Daten als solchen. Daher kann von diesen Spezialtatbeständen nicht auf ein umfassendes „Dateneigentum“ geschlossen werden.

Aus dieser Feststellung folgt, dass der Schutz und der Handel mit Daten - von Sonderfällen abgesehen - in der Breite nur durch eine Kombination aus vertraglichen Vereinbarungen und dem faktischen Zugang beziehungsweise Ausschluss des Zugangs zu den Daten realisierbar sind. ${ }^{194}$ Inwiefern und wem Daten faktisch (un)zugänglich sind, hängt unter anderem (auch) von den Eigentumsverhältnissen an dem Speichermedium ab, auf welchem die Daten abgespeichert sind. Wem das Sacheigentum am Speichermedium zusteht, bestimmt sich indes allein nach klassischen sachenrechtlichen Maßstäben und darf nicht mit der soeben dargestellten Debatte zum „Dateneigentum“ vermengt werden. ${ }^{195}$

\section{Ausblick: Rechtspolitische Diskussion}

Im Anschluss an die Debatte zum geltenden Recht wird derzeit eine lebhafte rechtspolitische Diskussion darüber geführt, ob und inwieweit es sinnvoll und notwendig ist, der gegenwärtigen Rechtslage mit der Einführung eines eigentumsähnlichen oder in sonstiger Weise spezifischen Datenrechts zu begegnen. Ein wichtiger Teilaspekt dieser Auseinandersetzung ist beispielsweise die Frage des Berechtigten.

194 Diese Einschätzung teilt Markendorf, ZD 2018, 409, 410 und nimmt sie zum Anlass, den Einsatz der Blockchain-Technologie als Ausgangspunkt einer Datenrechtsordnung vorzuschlagen.

195 Vgl. dazu Boehm, ZEuP 2016, 358, 384 f. Entsprechend sind auch die Frage nach dem Erbrecht an Daten und dem Erbrecht an dem körperlichen Speichermedium zu trennen. 
Diese Arbeit bietet nicht den Rahmen, um einen umfassenden Überblick über die rechtspolitische Diskussion zu geben. ${ }^{196}$ Es sei lediglich auf die Diskrepanz der Positionen des europäischen und des deutschen Gesetzgebers hingewiesen. Während die Arbeitsgruppe „Digitaler Neustart“ der Konferenz der Justizministerinnen und Justizminister der Länder momen$\tan$ im Wesentlichen keinen Reformbedarf sieht, ${ }^{197}$ zeigen die europäischen Institutionen mehr Handlungsbereitschaft. So führte die europäische Kommission in ihrer „Strategie für einen digitalen Binnenmarkt in Europa"198 schon im Jahr 2015 unter Ziff. 4.1 aus, dass sie den Aufbau einer Datenwirtschaft beabsichtige und als Teil dessen auch auf „die neuen Fragen des Eigentums an Daten [...] eingehen" werde. Im Anschluss daran kündigte die europäische Kommission 2017 in ihrer Mitteilung zum „Aufbau einer europäischen Datenwirtschaft ${ }^{\text {"199 }}$ an, ein Recht des Datenerzeugers an nicht-personenbezogenen Daten erörtern zu wollen. Parallel dazu sehen einige Autoren auch im Richtlinienentwurf über bestimmte vertragsrechtliche Aspekte der Bereitstellung digitaler Inhalte, der (personenbezogene) Daten als Gegenleistung im vertraglichen Synallagma vorsieht, ${ }^{200}$ einen potenziellen Anknüpfungspunkt für den Rechtehandel mit (personenbezogenen) Daten. ${ }^{201}$ Schließlich ist hinzuweisen auf die Verordnung über einen Rahmen für den freien Verkehr nicht-personenbezogener Daten, ${ }^{202}$ durch welche die Interoperabilität und Portabilität von nichtpersonenbezogenen Daten gefördert werden soll. Dem entspricht für personenbezogene Daten der Regelungsgehalt und Telos von Art. 20 DS-

196 Hingewiesen sei aber auf den wirtschaftswissenschaftlichen Beitrag zur Debatte von Kerber, GRUR Int. 2016, 989, der ,sowohl aus theoretischer als auch empirischer Perspektive" keine Hinweise sieht, welche die Einführung eines immaterialgüterrechtlichen Datenrechts begründen könnten.

197 Bericht der Arbeitsgruppe „Digitaler Neustart“ der Konferenz der Justizministerinnen und Justizminister der Länder, S. 97 f. sowie Kapitel 1, passim, S. 29-98, abrufbar a.a.O. (Fn. 43).

198 Mitteilung der Kommission vom 6. Mai 2015, Strategie für einen digitalen Binnenmarkt für Europa, COM [2015] 192 final. Dazu u.a. Zech, GRUR 2015, 1151, $1151 \mathrm{ff}$.

199 Mitteilung der Kommission vom 10. Januar 2017, Aufbau einer europäischen Datenwirtschaft, COM [2017] 9 final, S. 14. Dazu u.a. Paal/Hennemann, NJW 2017, 1697, 1698.

200 S. dazu bereits oben die Nachweise in Fn. 75.

201 Paal/Hennemann, NJW 2017, 1697, 1698; Röttgen in: Specht-Riemenschneider/ Werry/Werry (Hrsg.), Datenrecht in der Digitalisierung, S. $390 \mathrm{ff}$.

202 VO (EU) 2018/1807 des Europäischen Parlaments und des Rates vom 14. November 2018 über einen Rahmen für den freien Verkehr nicht-personenbezogener Daten in der Europäischen Union. 
GVO. ${ }^{203}$ Nicht zuletzt bestimmt Art. 1 DS-GVO als Gegenstand und Ziel der Verordnung neben dem Recht auf Schutz personenbezogener Daten in Art. 1 Abs. 1 und 3 DS-GVO auch den freien Verkehr personenbezogener Daten im europäischen Binnenmarkt. ${ }^{204}$ Dies kann als Programmsatz verstanden werden, der eine Offenheit für die perspektivische Formierung von Datenrechten signalisiert, die in einem freien Binnenmarkt handelbar wären.

Auch wenn sich hier noch längst kein einheitliches Bild abzeichnet und die Rechtsentwicklung für personenbezogene und nicht-personenbezogene Daten (naturgemäß) nicht identisch verläuft, ist in Brüssel in jedem Fall ein größerer Gestaltungswille zu konstatieren, als ihn der nationale Gesetzgeber in Deutschland bislang zeigt. Die weitere Entwicklung bleibt abzuwarten.

IV. Rückschlüsse aus der Diskussion für den digitalen Nachlass

Aspekte eines möglichen Datenrechts wie etwa die rechtliche Qualität und Fassbarkeit von Daten, ihre Inhaberschaft und die Gewährleistung ihres Schutzes werden vor dem Hintergrund der gesamtgesellschaftlichen Bedeutung der Digitalisierung auf eine große Vielzahl von Rechtsgebieten einwirken. Das zeigt sich exemplarisch am Fall des digitalen Nachlasses. Dessen Ausgangspunkt ist die genaue Bestimmung des Objekts des Erbgangs. In diesem Kontext kann nicht gleichsam inzidenter über die weitreichenden Fragen eines etwaigen Zuordnungsregimes für Daten entschieden werden. ${ }^{205}$ Aber auch und gerade in Ansehung der Reformbestrebungen auf europäischer Ebene muss die weitere Rechtsentwicklung hier aufmerksam verfolgt werden, da eine (Neu-)Regelung der rechtlichen Qualität oder Zuordnung von Daten auf die Bestimmung des Erbrechtsobjekts zurückwirken dürfte. Für das geltende Recht gilt es nach alledem, zumindest einstweilen eine „Verschuldrechtlichung [statt einer] Verdinglichung

203 Zu Sinn und Zweck von Art. 20 DS-GVO vgl. Paal in: Paal/Pauly (Hrsg.), DSGVO, Art. 20 Rn. 4-6.

204 Pötters in: Gola (Hrsg.), DS-GVO, Art. 1 Rn. 5 f. bezeichnet die beiden Zielbestimmungen als grds. gleichrangig; zum Grundsatz des freien Datenverkehrs innerhalb der Europäischen Union vgl. Zerdick in: Ehmann/Selmayr (Hrsg.), Art. 1 DS-GVO Rn. 9 ff. m.w.N.

205 Sorge, MMR 2018, 372, 377. 
der Universalsukzession $2.0^{\text {“206 }}$ zu konstatieren. Infolgedessen wird der Fokus im Bereich des digitalen Nachlasses darauf liegen müssen, ob und inwieweit vertragliche Rechte und Pflichten des Erblassers vererblich sind. ${ }^{207}$

\section{Zwischenergebnis zu Kapitel 2}

Die Rechtsobjekte, die in den digitalen Nachlass fallen, sind disparat. Das gilt zumindest, solange und soweit kein Recht an Daten anerkannt oder vom Gesetzgeber geschaffen wird. ${ }^{208}$ Die (rechtspolitische) Debatte zum Recht an Daten sowie insbesondere ein entsprechendes legislatives Tätigwerden können indes (auch unbeabsichtigt) Folgen für den Umgang mit dem digitalen Nachlass haben. Das gilt vor allem für die Genese neuer Rechtsobjekte sowie für die Entscheidung darüber, wem und unter welchen Voraussetzungen das etwaige Recht zuzuordnen ist.

Für den weiteren Verlauf der Untersuchung ist auf Grundlage des geltenden Rechts die nutzungsvertragliche Position des Erblassers der entscheidende Anknüpfungspunkt für die Fragen nach der Übergangsfähigkeit und Durchsetzbarkeit des digitalen Erbes. Mit der großen Diversität providerseitiger Informations- und Kommunikationsangebote korrespondierte dabei eine Vielfalt der Nutzungsverträge. Deren jeweilige Spezifika sind einzelfallgerecht bei der folgenden erb-, schuld- und datenschutzrechtlichen Untersuchung zu berücksichtigen. Daneben stehen die Urheber- und Leistungsschutzrechte des Erblassers an selbstgenerierten Accountinhalten als eigenständiges Erbrechtsobjekt.

206 Kritisch so Sorge, MMR 2018, 372, 377; s. aber die Studie des Fraunhofer Instituts für Sichere Informationstechnologie, S. 102, abrufbar a.a.O. (Fn. 7).

207 Das gilt trotz der im Schrifttum geäußerten Bedenken gegenüber einem hauptsächlich vertraglich organisiertem Ordnungsregime für Daten: Faust, Gutachten zum 71. DJT, 2016, S. A 77 ff. sieht derzeit einen unvollständigen Integritätsschutz von Daten; Sorge, MMR 2018, 372, 377 bezeichnet den Ausgangspunkt des digitalen Nachlasses als wenig zukunftsträchtig, da schuldrechtliche Ansprüche schon strukturell prekär seien; Grützmacher, CR 2016, 485, 492 und 495 weist hin auf die Anfälligkeit vertraglicher Konstruktionen in Fällen von Insolvenz. Zum Ganzen - auch mit Gegenargumenten - vgl. Bericht der Arbeitsgruppe „Digitaler Neustart“ der Konferenz der Justizministerinnen und Justizminister der Länder, S. 29 ff., 90 f., abrufbar a.a.O. (Fn. 43).

208 Anders Thiesen, Daten in der Erbmasse, S. 182 nach dem „eine Auseinandersetzung mit möglichen Sonderrechten an Daten nicht die Lücke schließen [kann], die bei einer rein vertragsrechtlichen Herangehensweise entsteht.“ 


\section{Kapitel 3 - Die Rechtsverhältnisse zwischen Provider und Nutzer im Nachlass}

Nachdem die relevanten Erbrechtsobjekte bestimmt wurden, ist der Blick nun auf ihre (Un-)Vererbbarkeit sowie auf etwaige Beschränkungen des übergegangenen Rechts zu richten. Angesichts der Tatsache, dass die Digitalwirtschaft mehr noch als das globalisierte Wirtschaftsleben im Allgemeinen durch das grenzüberschreitende Agieren aller beteiligten Akteure geprägt ist, sind internationalprivatrechtliche Vorüberlegungen zum anwendbaren Recht angezeigt (dazu A.). Im Anschluss sind die aufgeworfenen Fragen betreffend die Übergangsfähigkeit und Durchsetzbarkeit am Maßstab des deutschen Rechts zuerst für die nutzungsvertragliche Position des Erblassers (dazu B.) und sodann für dessen Immaterialgüterrechte (dazu C.) zu untersuchen.

\section{A. Vorüberlegung: Anwendbares Recht /IPR}

Der Analyse des materiellen deutschen Rechts sind einführende Überlegungen zur internationalprivatrechtlichen Frage nach dem anwendbaren Recht voranzustellen. ${ }^{209}$ Denn insbesondere Sachverhalte, die einen Bezug zur Digitalisierung oder zum Internet haben, sind vielfach durch grenzüberschreitende Elemente geprägt. ${ }^{210}$ Sowohl im vertraglichen - genannt sei beispielsweise der Fernabsatz im Online-Handel - als auch im außerver-

209 Diese Arbeit soll sich auf das materielle Recht konzentrieren. Zur internationalzivilverfahrensrechtlichen Frage nach der gerichtlichen Zuständigkeit im Kontext des digitalen Nachlasses vgl. Rott/Rott, NWB-EV 2013, 160, 161 f.; Bock in: Groll/Steiner (Hrsg.), Praxishandbuch Erbrechtsberatung, Rn. 20.149 ff.; Herzog/ Pruns, Der digitale Nachlass in der Vorsorge- und Erbrechtspraxis, S. $123 \mathrm{ff}$.; der BGH begründet die Zuständigkeit deutscher Gerichte in seinem Facebook-Urteil mit Art. 17 Abs. 1 lit.c), Abs. 2 Brüssel Ia-VO in Verbindung mit Art. 18 Abs. 1 Var. 2 Brüssel Ia-VO (Internationaler Gerichtsstand für Verbrauchersachen am Wohnsitz des Verbrauchers), vgl. BGH NJW 2018, 3178 Rn. 16.

210 Mit anschaulichen Beispielen Härting, Internetrecht, Rn. 2760 f.; Hoeren, Internetrecht, Rn. 1245; Hetmank, Internetrecht; S. 11; in diesem Sinne aber zum Internationalen Zivilverfahrensrecht (IZVR) Banholzer in: Hoeren/Sieber/Holznagel (Hrsg.), MMR-HdB, Teil 25 Rn. 10-12 m.w.N. 
traglichen Bereich - so etwa bei Persönlichkeitsrechtverletzungen in den sozialen Medien - agieren Verbraucher wie auch Unternehmen in digitalen Zusammenhängen häufig über nationalstaatliche Grenzen hinweg. Daraus folgt die kollisionsrechtliche Bedeutung dieser Fälle. ${ }^{211}$ Anhand allfälliger Rechtsstreite im Kontext des digitalen Nachlasses zeigt sich diese internationalprivatrechtliche Dimension auf paradigmatische Weise. ${ }^{212}$

Wie sich gezeigt hat, verbirgt sich hinter der Bezeichnung des digitalen Nachlasses eine große Bandbreite unterschiedlicher Erbrechtsobjekte. ${ }^{213}$ Diese Vielfalt der Erbrechtsobjekte wirkt in die kollisionsrechtliche Anknüpfung fort. Im Vordergrund steht hier, wie auch im materiellen Recht, die Untersuchung vertraglicher Rechtspositionen (dazu II.). Daneben soll auf das anwendbare Recht bei anderen Erbrechtsobjekten, namentlich Immaterialgüterrechten, eingegangen werden (dazu III.). Alledem ist eine Abgrenzung zu den Vorgaben des internationalen Erbrechts voranzustellen (dazu I.).

\section{Ausgangspunkt: Internationales Erbrecht}

Die EuErbVO 214 enthält das für die Rechtsnachfolge von Todes wegen relevante Kollisionsrecht für alle Erbfälle, die nach dem 16. August 2015 eingetreten sind, vgl. Art. 1 Abs. 1, 83 f. EuErbVO sowie Art. 3 Nr. 1 lit. e), 25 EGBGB. Als zentrale internationalprivatrechtliche Norm der Verordnung unterwirft Art. 21 Abs. 1 EuErbVO „die gesamte Rechtsnachfolge von To-

211 Nach der Legaldefinition des Art. 3 EGBGB „bestimmt sich das anzuwendende Recht bei Sachverhalten mit einer Verbindung zu einem ausländischen Staat“ nach dem IPR. Siehe dazu Looschelders in: Staudinger, Einleitung zum IPR, Rn. 4, 13.

212 Dem widmen Herzog/Pruns, Der digitale Nachlass in der Vorsorge- und Erbrechtspraxis, S. $123 \mathrm{ff}$. folgerichtig eigens einen Abschnitt in ihrem Praxishandbuch; Bock in: Groll/Steiner (Hrsg.), Praxishandbuch Erbrechtsberatung, Rn. 20.141 ff.; Kutscher, Der digitale Nachlass, S. 67 ff.; Budzikiewicz, AcP (218) 2018, 558, 588 f. speziell zum Zusammenhang zwischen IPR und AGB; Bericht der Arbeitsgruppe „Digitaler Neustart“ der Konferenz der Justizministerinnen und Justizminister der Länder, S. 403 f., abrufbar a.a.O. (Fn. 43).

213 S. hierzu bereits die Nachweise in Fn. 5, 6 und 7.

214 Verordnung (EU) Nr.650/2012 des Europäischen Parlaments und des Rates vom 4. Juli 2012 über die Zuständigkeit, das anzuwendende Recht, die Anerkennung und Vollstreckung von Entscheidungen und die Annahme und Vollstreckung öffentlicher Urkunden in Erbsachen sowie zur Einführung eines Europäischen Nachlasszeugnisses, Celex-Nr. 32012 R 0650. 
des wegen dem Recht des Staates, in dem der Erblasser im Zeitpunkt seines Todes seinen gewöhnlichen Aufenthalt hatte." 215 Damit wendet sich die EuErbVO von dem in Deutschland zuvor maßgeblichen Anknüpfungsmoment der Staatsbürgerschaft des Erblassers weitgehend ab, vgl. noch Art. 25 Abs. 1 EGBGB a.F. ${ }^{216}$

Aus der nicht abschließenden Aufzählung des Art. 23 Abs. 2 EuErbVO ergibt sich, was als ein Bestandteil der „gesamten Rechtsnachfolge von Todes wegen “ dem Umfang des Erbstatuts zuzurechnen ist. ${ }^{217}$ Dazu gehören insbesondere der Eintritt und Zeitpunkt des Erbfalls, ${ }^{218}$ die Berufung der Berechtigten $^{219}$ und die Erbfähigkeit ${ }^{220}$ als klassische Fragen des Erbrechts.

Dagegen richtet sich die Vererbbarkeit einer Rechtsposition nicht nach dem Erbstatut, sondern nach dem jeweiligen Statut des betreffenden Rechtsverhältnisses. ${ }^{221}$ Denn das letztere Statut befindet über den Inhalt des infrage stehenden Rechts und damit auch über die Dauer seiner Existenz. ${ }^{222}$ Ferner setzt der Wortlaut des Art. 23 Abs. 2 lit. e) EuErbVO die Existenz von zum Nachlass gehörenden Gegenständen für den Übergang auf die Erben voraus. ${ }^{223}$ Für schuldrechtliche Rechtsverhältnisse folgt das ferner aus Art. 14 Abs. 2 Rom I-VO, dessen Rechtsgedanke neben der Abtretung auch die Vererbbarkeit erfasst, sowie explizit aus Art. 15 lit. e) Rom II-VO. ${ }^{224}$ Die Systematik des materiellen deutschen Rechts verhält sich in-

215 Vgl. dazu Köhler in: NK-Nachfolgerecht, Art. 21 EuErbVO Rn. 3 ff.; Bauer in: Dutta/Weber (Hrsg.), Internationales Erbrecht, Art. 21 EuErbVO Rn. 3 ff.

216 Köbler in: NK-Nachfolgerecht, Art. 21 EuErbVO Rn. 1; Bauer in: Dutta/Weber (Hrsg.), Internationales Erbrecht, Art. 21 EuErbVO Rn. 1. Die Staatsbürgerschaft ist nunmehr nur noch im Rahmen einer beschränkten Rechtswahlmöglichkeit des Erblassers von Bedeutung, vgl. Art. 22 Abs. 1 EuErbVO.

217 Der Wortlaut des Art. 23. Abs. 2 EuErbVO ist insoweit eindeutig (,insbesondere“). So auch Köhler in: NK-Nachfolgerecht, Art. 23 EuErbVO Rn. 1; Dutta in: MüKo BGB, Art. 23 EuErbVO Rn. 5.

218 Vgl. Art. 23. Abs. 2 lit. a) EuErbVO. Siehe dazu Köhler in: NK-Nachfolgerecht, Art. 23 EuErbVO Rn. 2.

219 Vgl. Art. 23. Abs. 2 lit. b) EuErbVO. Dazu Köhler a.a.O. (Fn. 218), Rn. 3.

220 Vgl. Art. 23. Abs. 2 lit. c) EuErbVO. Dazu Köhler a.a.O. (Fn. 218), Rn. 4.

221 Schmidt in: Dutta/Weber (Hrsg.), Internationales Erbrecht, Art. 1 EuErbVO Rn. 145 f.; Dutta in: MüKo BGB, Art. 23 EuErbVO Rn. 25 und Art. 1 EuErbVO Rn. 37, 54 jeweils m.w.N.; Bock in: Groll/Steiner (Hrsg.), Praxishandbuch Erbrechtsberatung, Rn. 20.143; zur Abgrenzung von Erb- und Gesellschaftsstatut so auch Köhler in: NK-Nachfolgerecht, Art. 23 EuErbVO Rn. 22.

222 Schmidt in: Dutta/Weber (Hrsg.), Internationales Erbrecht, Art. 1 EuErbVO Rn. 145.

223 Bock in: Groll/Steiner (Hrsg.), Praxishandbuch Erbrechtsberatung, Rn. 20.143.

224 Dutta in: MüKo BGB, Art. 23 EuErbVO Rn. 25. 
soweit parallel; das fünfte Buch des BGB enthält die erbrechtlichen Vorgaben u.a. zu Erbfall, Erbfolge und Erbfähigkeit. Die Frage nach der Vererbbarkeit wird dagegen im Kontext des jeweiligen Rechtsverhältnisses geregelt, wenn und soweit der Gesetzgeber hier ein Klarstellungs- oder Regelungsbedürfnis sieht. ${ }^{225}$

Daraus folgt für diese Arbeit, dass kollisions- ebenso wie materiell-rechtlich nach dem konkret infrage stehenden Erbrechtsobjekt zu unterscheiden ist. So entscheidet das Sachstatut über die Vererbbarkeit eines körperlichen Speichermediums, wie etwa einer Festplatte. Das Vertragsstatut entscheidet über die Vererbbarkeit der Rechte und Pflichten aus dem Nutzungsvertrag zwischen den Nutzern und Providern. ${ }^{226}$ Schließlich richtet sich die Vererbbarkeit von Immaterialgüterrechten nach dem auf sie anwendbaren Statut. ${ }^{227}$

\section{Vertragsstatut bei Nutzungsverträgen als Erbrechtsobjekt}

Die Rom I-VO regelt ausweislich ihres Art. 1 Abs. 1 Rom I-VO das anwendbare Recht „für vertragliche Schuldverhältnisse in Zivil- und Handelssachen." Im Folgenden wird in einem ersten Schritt zwischen Verbraucherverträgen im Sinne von Art. 6 Rom I-VO und sonstigen Verträgen zu differenzieren sein. In beiden Kategorien ist sodann danach zu unterscheiden, ob die Parteien eine Rechtswahl getroffen haben - und bejahendenfalls ob sie deutsches Recht gewählt haben - oder nicht. Die Darstellung wird sich hierbei auf Aspekte konzentrieren, die besondere Herausforderungen im kollisionsrechtlichen Umgang gerade mit dem digitalen Nachlass begründen. ${ }^{228}$

225 Genannt seien etwa $₫ 1061$ S. 1 BGB für den Nießbrauch sowie $₫ 613$ BGB für den Dienstvertrag, der nach einhelliger Meinung auch die (Nicht-)Vererbbarkeit der Dienstpflicht regelt. Hingewiesen sei ferner auf $\$ 28$ Abs. 1 UrhG. Zu den Vorschriften, welche die Vererbbarkeit im Rahmen verschiedener Gesellschaftsformen regeln, s. Scherer in: Sudhoff (Hrsg.), Unternehmensnachfolge, Kap. A Rn. 9 ff.

226 Zur autonomen internationalprivatrechtlichen Qualifikation des Nutzungsverhältnisses als Vertrag vgl. Gläser, MMR 2015, 699, 699.

227 Schmidt in: Dutta/Weber (Hrsg.), Internationales Erbrecht, Art. 1 EuErbVO Rn. 145 f.; Dutta in: MüKo BGB, Art. 1 EuErbVO Rn. 54.

228 Jenseits dieser Aspekte sei v.a. auf die vorhandene internationalprivatrechtliche Literatur zur Rom I-VO und insbesondere zu Art. 6 Rom I-VO verwiesen, s. die Nachweise bei der folgenden Darstellung der einzelnen Tatbestandsmerkmale. 
1. Nutzungsverträge als Verbraucherverträge i.S.v. Art. 6 Rom I-VO

Art. 6 Rom I-VO regelt das auf Verbraucherverträge anzuwendende Recht. Dabei erfasst Art. 6 Abs. 1 Rom I-VO den Fall, dass die Parteien keine Rechtwahl getroffen haben. Art. 6 Abs. 2 Rom I-VO statuiert das Günstigkeitsprinzip für Verträge, die einer (wirksamen) ${ }^{229}$ Rechtswahl unterliegen.

\section{a) Tatbestand des Art. 6 Abs. 1 Rom I-VO}

Voraussetzung für die Eröffnung des persönlichen Anwendungsbereichs von Art. 6 Rom I-VO ist, dass „eine natürliche Person zu einem Zweck, der nicht ihrer beruflichen oder gewerblichen Tätigkeit zugerechnet werden kann (,Verbraucher'), mit einer anderen Person einen Vertrag schließt, die in Ausübung ihrer beruflichen oder gewerblichen Tätigkeit handelt (,Unternehmer'),"vgl. Art. 6 Abs. 1 Rom I-VO. ${ }^{230}$ Ohne eine privatautonome Rechtswahl unterliegt ein solcher Vertrag „dem Recht des Staates, in dem der Verbraucher seinen gewöhnlichen Aufenthalt hat," sofern der Unternehmer seine berufliche oder gewerbliche Tätigkeit in dem Aufenthaltsstaat des Verbrauchers ausübt oder sie (auch) auf diesen Staat ausrichtet. ${ }^{231}$

\section{aa) Der Nutzer als Verbraucher}

Die verschiedenen Internetdienste der Provider erlauben hierbei Vertragsund Nutzungskonstellationen, die sich zwischen reinen Verbraucherverträgen, Mischverträgen und rein beruflich genutzten Angeboten bewegen. Manche Internetdienste fallen im Ganzen in eine dieser Kategorien. Von Ausnahmen in Einzelfällen abgesehen, dürfte zum Beispiel die Nutzung von Berufsnetzwerken wie LinkedIn und Xing beruflichen oder gewerbli-

229 Eine unwirksame Rechtswahl wird wie ein Vertrag ohne Rechtswahl - also nach Art. 6 Abs. 1 Rom I-VO - behandelt, vgl. Thorn in: Palandt, Art. 6 Rom IVO Rn. 10; Bach in: Spindler/Schuster (Hrsg.), Recht der elektronischen Medien, Art. 6 Rom I-VO Rn. 27.

230 Zum Verbraucherbegriff des Art. 6 Rom I-VO s. Martiny in: MüKo BGB, Art. 6 Rom I-VO Rn. 12 ff.; Magnus in: Staudinger, Art. 6 Rom I-VO Rn. 38 ff.

$231 \mathrm{Zu}$ den situativen Anwendungsvoraussetzungen vgl. Martiny in: MüKo BGB, Art. 6 Rom I-VO Rn. 36 ff.; Magnus in: Staudinger, Art. 6 Rom I-VO Rn. 102 ff. Siehe dazu ferner die Erwägungsgründe 24 und 25 der Rom I-VO. 
chen Zwecken dienen, während Nutzungsverträge mit Online-Datingportalen regelmäßig als Verbraucherverträge zu qualifizieren sind. E-MailDienste werden in vielen Fällen sowohl beruflich als auch privat genutzt. $^{232}$

Bei anderen Internetdiensten dürfte demgegenüber erst die konkrete Art der Nutzung über die hier aufgeworfene Frage entscheiden. So teilt zwar die Mehrzahl der Nutzer von Instagram ihre Fotos in ihrer Rolle als Verbraucher. Sogenannte Influencer hingegen können (auch) gewerblich handeln. Das Influencer-Marketing steht hier beispielhaft für Abgrenzungsfragen in inhaltlicher wie zeitlicher Hinsicht: Erfolgreiche Influencer mit über einer Million Follower ${ }^{233}$ handeln professionell und können dabei mit hohen Umsätzen rechnen. ${ }^{234}$ Der Weg zu diesem Status verläuft indes graduell und schließt mitunter Übergangsstadien ein. ${ }^{235}$ In diesem Zusammenhang wird auch von sogenannten Micro-Influencern gesprochen. Schließlich gibt es Nutzer, die (noch) rein privat handeln, aber den Status als Influencer anstreben. ${ }^{236}$ Werbepartner platzieren ihre Produkte nach Vereinbarung in den Beiträgen reichweitestarker Accounts. Die Gegenleistung der werbenden Unternehmen, soweit eine solche überhaupt erbracht wird, reicht von der kostenfreien Zurverfügungstellung der abgebildeten

232 Ähnlich Gläser, MMR 2015, 699, 702 und Kutscher, Der digitale Nachlass, S. 78.

233 Zur Veranschaulichung sei auf die Instagram-Accounts des Fußballspielers Cristiano Ronaldo (242 Mio. Abonnenten) und der Musikerin Ariana Grande (206 Mio. Abonnenten) verwiesen, deren Profile zu denen mit den meisten sog. „Followern“ auf Instagram gehören [Stand: 17.11.2020].

234 Laut Willems, MMR 2018, 707, 707 erzielte die deutsche Influencerin Caro Daur im Jahr 2017 einen Jahresumsatz von EUR 1 Mio. Weiterführend zur rechtlichen Beurteilung des Instagram-Marketings s. etwa Fries, Influencer-Marketing. Informationspflichten bei Werbung durch Meinungsführer in Social Media, passim; Leeb/Maisch, ZUM 2019, 29; Köhler, ZUM-RD 2019, 141; Troge, GRURPrax 2018, 87.

235 Generalanwalt Bobek konstatiert in seinen Schlussanträge vom 14.11.2017 C-498/16, Rdnr. 49 - Maximilian Schrems/Facebook Ireland Lim.: Es gibt „heutzutage ganze Berufszweige, die die Grenze zwischen privaten und beruflichen Verbindungen in der Internet-Kommunikation, insbesondere in sozialen Netzwerken, verwischen. Einige Nutzungen könnten als privat erscheinen, sind aber in vollem Umfang kommerzieller Natur. „Influencer“, die Marketing in sozialen Medien betreiben, „Prosumer“ (professionelle Verbraucher) oder Community Manager nutzen ihre persönlichen Konten in sozialen Netzwerken [...] als wichtiges Arbeitswerkzeug." S. dazu Willems, MMR 2018, 707, $707 \mathrm{f}$.

236 Zur Phänomenalisierung des Influencer-Marketings mit Angaben zu Umsätzen in Abhängigkeit zur Anzahl der Follower, vgl. Fries, Influencer-Marketing. Informationspflichten bei Werbung durch Meinungsführer in Social Media, S. $3 \mathrm{ff}$. 
Produkte bis hin zur Zahlung von Honoraren. Die Übergänge sind fließend und mitunter zufällig, weil sie unter anderem vom Verhandlungsgeschick der Beteiligten abhängen. Insgesamt wird hierdurch eine inhaltliche Kategorisierung erschwert.

Die Rechtsprechung zum Internationalen Zivilverfahrensrecht gibt dazu Anlass, von einer engen Auslegung des Verbraucherbegriffs in Fällen einer Mischnutzung auszugehen. So kommen die Vorschriften zum Gerichtsstand für Verbrauchersachen (nur) dann zur Anwendung, wenn ein etwaiger beruflich-gewerblicher Zweck des Geschäfts derart nebensächlich ist, dass er eine „ganz untergeordnete Rolle spielt.“" ${ }^{237}$ Es ist umstritten, ob diese Rechtsprechung auf das Europäische Kollisionsrecht übertragen werden kann. ${ }^{238}$ Dagegen wird zwar angeführt, dass dies zu einer Einschränkung des durch Art. 6 Rom I-VO vermittelten Verbraucherschutzes führen würde. ${ }^{239}$ Allerdings ähneln sich der Wortlaut von Art. 17 Abs. 1 Brüssel Ia-VO und Art. 6 Abs. 1 Rom I-VO stark, woraus sich ein überzeugendes systematisches Argument für die einheitliche Auslegung der europäischen Rechtsbegriffe ableiten lässt. ${ }^{240}$ Danach kommen richtigerweise nur die Nutzer in den Genuss des Verbraucherstatuts nach Art. 6 Rom I-VO, die ihren Account (nahezu) ausschließlich für private Zwecke nutzen.

Die Gewichtung von beruflichen und privaten Zwecken wird nicht anhand der inneren Willensrichtung des Nutzers bestimmt, sondern auf Grundlage der für den Provider objektiv erkennbaren Umstände des Geschäfts. ${ }^{241}$ Der für diese Feststellung maßgebliche Zeitpunkt ist nach geltendem Recht der Moment des Vertragsschlusses. Ändert der Zweck des Geschäfts sich später, soll das den privaten oder geschäftlichen Charakter

237 So ausdrücklich BGH, Beschluss vom 13.10.2016 - IX ZB 9/16, BeckRS 2016, 18608 Rn. 8 in Anknüpfung an EuGH NJW 2005, 653 Rn. 39 ff.

238 Ausführlich zum Streitstand Magnus in: Staudinger, Art. 6 Rom I-VO Rn. 47 m.w.N.; Staudinger in: HK-BGB, Art. 6 Rom I-VO Rn. 5 m.w.N.; offenlassend Martiny in: MüKo BGB, Art. 6 Rom I-VO Rn. 15.

239 Magnus in: Staudinger, Art. 6 Rom I-VO Rn. 47.

240 Thorn in: Palandt, Art. 6 Rom I-VO Rn. 5; Ragno in: Leible/Ferrari (Hrsg.), Rome I Regulation, S. 135 f.; Stürner in: Erman, Art. 6 Rom I-VO Rn. 8 f.; Kutscher, Der digitale Nachlass, S. 78 f., weist allerdings auf Erw. Gr. 17 der VR-RL hin, wonach eine Schwerpunktbetrachtung vorzunehmen ist. Anschließend plädiert Kutscher selbst aber für ein Wahlrecht des Nutzers.

241 Thorn in: Palandt, Art. 6 Rom I-VO Rn. 5; Ragno in: Leible/Ferrari (Hrsg.), Rome I Regulation, S. 134 f.; mit Beispielen für objektiv erkennbare Indizien Kutscher, Der digitale Nachlass, S. 78. 
der Transaktion nicht (mehr) berühren. ${ }^{242} \mathrm{Ob}$ diese Abgrenzung immer zu sachgemäßen Ergebnissen führt, erscheint vor dem Hintergrund des Influencings zumindest zweifelhaft. Denn ein Instagram- oder YouTube-Nutzungsvertrag kann zum Zeitpunkt des Vertragsschlusses noch ein Verbrauchervertrag gewesen sein und erst durch eine später entstandene Influencer-Tätigkeit gewerbliche Züge angenommen haben. ${ }^{243}$

Zusammenfassend sei festgestellt, dass aus der Vielfalt der Vertrags- und Nutzungskonstellationen durchaus komplexe Fragen zur Verbraucherrolle des Nutzers folgen können. Dessen ungeachtet handelt die Mehrzahl der Nutzer in sozialen Netzwerken sowie von sonstigen Kommunikationsund Informationsdiensten aus rein privaten Motiven und damit als Verbraucher.

bb) Ausüben bzw. Ausrichten der Tätigkeit durch den Anbieter

Der Provider muss seine beruflich-gewerbliche Tätigkeit in dem Staat des gewöhnlichen Aufenthalts des Verbrauchers ausüben oder sie (auch) auf diesen Staat ausrichten, vgl. Art. 6 Abs. 1 lit a) und b) Rom I-VO. Ferner muss der betreffende Vertrag in den Bereich dieser beruflich-gewerblichen Tätigkeit fallen. Letztere Voraussetzung erfüllen die durch die Provider angebotenen Nutzungsverträge grundsätzlich, da sie den Kern der geschäftlichen Tätigkeit der Provider gegenüber ihren Nutzern bilden.

Das Merkmal des Ausübens ist erfüllt, wenn der Unternehmer im Verbraucherstaat aktiv am Geschäftsverkehr teilnimmt. ${ }^{244}$ Eine Niederlassung des Providers in dem betreffenden Staat ist dafür zwar nicht zwingend notwendig, sofern vorhanden aber hinreichend. ${ }^{245}$ Unter anderem die Anbieter Facebook, Google, Twitter und LinkedIn betreiben Zweigniederlassun-

242 So ausdrücklich Magnus in: Staudinger, Art. 6 Rom I-VO Rn. 49; wohl auch Martiny in: MüKo BGB, Art. 6 Rom I-VO Rn. 17.

243 Ähnliches gilt für einen E-Mail-Account, der zunächst rein privat genutzt wurde und erst im Laufe der Zeit auch zu beruflichen Zwecken verwendet wird. Die umgekehrte Reihenfolge dürfte weniger problematisch sein, da die strukturelle Ungleichheit der Vertragspartner, welche den Verbraucherschutz begründet, gerade nicht vorlag und sich auch nicht nachträglich einstellen kann.

244 Magnus in: Staudinger, Art. 6 Rom I-VO Rn. 107. Das Merkmal des Ausübens entspricht der Formulierung in Art. 17 Abs. 1 lit. c) Brüssel Ia-VO und soll nach Erwägungsgrund 24 Rom I-VO auch in Übereinstimmung damit ausgelegt werden, vgl. Magnus a.a.O., Rn. 106; Stürner in: Erman, Art. 6 Rom I-VO Rn. 21.

245 Magnus in: Staudinger, Art. 6 Rom I-VO Rn. 107. 
gen, Xing und Parship haben ihren Unternehmenssitz in Deutschland. Damit fällt die Tätigkeit einiger wichtiger Marktteilnehmer bereits unter Art. 6 Abs. 1 lit a) Rom I-VO.

Die Tatbestandsalternative des Ausrichtens ist inhaltlich weiter gefasst und setzt das Entfalten werbender unternehmerischer Tätigkeit zum Zwecke des Abschlusses und der Durchführung von Verträgen im Verbraucherstaat voraus. ${ }^{246}$ Der entsprechende Wille des Unternehmers ist aus allen relevanten Umständen des Einzelfalls zu ermitteln. ${ }^{247}$ Die Praxis der Provider in Deutschland dürfte dabei in den meisten Fällen als ein Ausrichten zu werten sein. Denn die Anbieter der Kommunikations- und Internetdienste haben in der Mehrzahl deutschsprachige Webseiten mit einer deutschen Top-Level-Domain (.de), sie werben aktiv auf dem deutschen Markt um Nutzer und verwenden dabei AGB in deutscher Sprache. ${ }^{248}$

b) Rechtsfolge: Qualifikation abhängig von Rechtswahl

Wenn die Voraussetzungen des Art. 6 Abs. 1 Rom I-VO erfüllt sind, ist das anzuwendende Recht in Abhängigkeit davon zu bestimmen, ob die Parteien eine (wirksame) Rechtswahl getroffen haben. Ist dies nicht der Fall, so gilt das Recht des Staates, in dem der Verbraucher seinen gewöhnlichen Aufenthalt, d.h. seinen tatsächlichen Lebensmittelpunkt, ${ }^{249}$ hat, vgl. Art. 6 Abs. 1 Rom I-VO.

Unterliegt der Verbrauchervertrag einer wirksamen Rechtswahl, dann statuiert Art. 6 Abs. 2 S. 2 Rom I-VO das Günstigkeitsprinzip zugunsten

246 Magnus in: Staudinger, Art. 6 Rom I-VO Rn. 112; eingehend Staudinger in: Ferrari u.a. (Hrsg.), Internationales Vertragsrecht, Art. 6 Rom I-VO Rn. 47 ff.; zum Verhältnis des Ausrichtens zu Art. 17 Abs. 1 lit.c) Brüssel Ia-VO gilt das in Fn. 244 Gesagte entsprechend, vgl. Magnus a.a.O.; Stürner in: Erman, Art. 6 Rom I-VO Rn. 23.

247 Magnus in: Staudinger, Art. 6 Rom I-VO Rn. 113, 115 ff. Zu den Indizien im Bereich sozialer Medien und Internetdienste mit zahlreichen Beispielen Kutscher, Der digitale Nachlass, S. 81 f. und Gläser, MMR 2015, 699, 702.

248 Beispielhaft Gläser, MMR 2015, 699, 702 zu den Nutzungsbedingungen von Facebook in Deutschland; Magnus in: Staudinger, Art. 4 Rom I-VO Rn. 601 f., 609; Rott/Rott, NWB-EV 2013, 160, 162.

249 Nach Thorn in: Palandt, Art. 19 Rom I-VO Rn. 6 ist das der Ort, an dem die Person durch „familiäre, freundschaftliche, berufliche sowie wirtschaftliche Beziehungen sozial integriert ist.“ Ähnlich auch Stürner in: Erman, Art. 19 Rom I-VO Rn. 2; Ragno in: Leible/Ferrari (Hrsg.), Rome I Regulation, S. 151. 
des Verbrauchers. Es bleibt den Parteien nach Art. 6 Abs. 2 S. 1 i.V.m. Art. 3 Rom I-VO zwar unbenommen, eine andere Rechtsordnung als die des Verbraucherstaats zu wählen. Diese Rechtwahl darf indes nicht dazu führen, dass dem Verbraucher der Schutz entzogen wird, der ihm durch zwingendes Recht seines Aufenthaltsstaats gewährt wird. ${ }^{250}$ Sofern die Parteien nicht (ohnehin schon) deutsches Recht gewählt haben, kommt aus der Perspektive deutscher Verbraucher der Vorschrift des $\$ 307$ Abs. 2 Nr. 1 BGB an dieser Stelle besondere Bedeutung zu. Da die $\$ \mathbb{S} 305 \mathrm{ff}$. BGB zwingendes, verbraucherschützendes Recht sind, gehören sie zu den Normen, deren Schutzniveau nach Maßgabe von Art. 6 Abs. 2 S. 2 Rom I-VO nicht unterlaufen werden darf. ${ }^{251}$ Nach $\$ 307$ Abs. 2 Nr. 1 BGB wiederum darf mittels AGB nicht von wesentlichen Grundgedanken des in Deutschland geltenden Rechts abgewichen werden. ${ }^{252} \mathrm{Im}$ Ergebnis folgt daraus, dass das deutsche Recht auch bei wirksamer Wahl einer anderen Rechtsordnung inzident zum Prüfungsmaßstab wird. ${ }^{253}$ Das gilt auch für Normen, die - wie $\mathbb{} 1922$ Abs. 1 BGB - nicht (primär) verbraucherschützend sind, aber über $\int 307$ Abs. 2 Nr. 1 BGB verbraucherrelevant werden. ${ }^{254}$ Zur Anwendung kommt das Recht, das sich im Vergleich zwischen der verbraucherstaatlichen und der gewählten Rechtsordnung als für den Verbraucher günstiger herausstellt - also im Falle eines Rechtsstreits eher seinem Antrag entspricht. ${ }^{255}$

250 Zum Günstigkeitsprinzip gem. Art. 6 Abs. 2 S. 2 Rom I-VO Magnus in: Staudinger, Art. 6 Rom I-VO Rn. 137 ff.; Stürner in: Erman, Art. 6 Rom I-VO Rn. 16; Martiny in: MüKo BGB, Art. 6 Rom I-VO Rn. 58 ff.; Ragno in: Leible/ Ferrari (Hrsg.), Rome I Regulation, S. $152 \mathrm{f}$.

251 Magnus in: Staudinger, Art. 6 Rom I-VO Rn. 140.

$252 \mathrm{Zu} \rrbracket 307$ Abs. 2 Nr. 1 BGB vgl. nur Grüneberg in: Palandt, $₫ 307$ BGB Rn. $28 \mathrm{ff}$. m.w.N.

253 Kutscher, Der digitale Nachlass, S. 83; Herzog/Pruns, Der digitale Nachlass in der Vorsorge- und Erbrechtspraxis, S. 126 f.; Bock in: Groll/Steiner (Hrsg.), Praxishandbuch Erbrechtsberatung, Rn. 20.147.

254 Kutscher, Der digitale Nachlass, S. 83 und ihr folgend der Bericht der Arbeitsgruppe „Digitaler Neustart“ der Konferenz der Justizministerinnen und Justizminister der Länder, S. 403 f., abrufbar a.a.O. (Fn. 43); explizit zu einer Abweichung von $\mathbb{1} 1922$ Abs. 1 BGB als unangemessene Benachteiligung im Sinne von $\$ 307$ Abs. 2 Nr. 1 (und Nr. 2) BGB vgl. BGH NJW 2018, 3178 Rn. 30 f.

255 Stürner in: Erman, Art. 6 Rom I-VO Rn. 16a; Thorn in: Palandt, Art. 6 Rom I-VO Rn. 8; Magnus in: Staudinger, Art. 6 Rom I-VO Rn. 144. 
c) Zwischenergebnis

Der Nutzungsvertrag zwischen einem Provider und einem Verbraucher, der seinen gewöhnlichen Aufenthalt in Deutschland hat, unterliegt deutschem Recht, sofern keine (wirksame) Rechtswahl vorliegt oder die Parteien wirksam deutsches Recht gewählt haben. Haben die Parteien hingegen wirksam eine andere Rechtsordnung als die deutsche gewählt, kommen die Bestimmungen der fremden Rechtsordnung nur insofern zur Anwendung, wie sie nicht hinter dem Niveau des deutschen Verbraucherschutzes zurückbleiben.

\section{Nutzungsverträge ohne Verbraucherbeteiligung}

Falls ein Nutzungsvertrag ohne Verbraucherbeteiligung und damit außerhalb des Anwendungsbereichs von Art. 6 Rom I-VO vorliegt, ${ }^{256}$ ist ebenfalls danach zu unterscheiden, ob die Parteien eine Rechtswahl getroffen haben. Ist das nicht der Fall, kommt es zur objektiven Grundanknüpfung nach Art. 4 Rom I-VO (dazu a), ${ }^{257}$ anderenfalls ist insbesondere Art. 3 Rom I-VO maßgeblich (dazu b).

a) Allgemeine Vertragskollisionsnorm, Art. 4 Rom I-VO

Die objektive Anknüpfung folgt dem durch Erwägungsgrund 19 und Art. 4 Rom I-VO vorgegebenen Dreischritt: Kann der anzuknüpfende Vertrag einer der ausdrücklich genannten Vertragsarten zugeordnet werden, gilt die vertragsspezifische Vorgabe des Art. 4 Abs. 1 Rom I-VO. Anderenfalls gibt nach Art. 4 Abs. 2 Rom I-VO die für den Vertrag charakteristische Leistung den Ausschlag. Die Anknüpfung nach Abs. 1 und Abs. 2 gilt dabei jeweils vorbehaltlich der Ausweichklausel des Art. 4 Abs. 3 Rom I-VO. Nur wenn keines dieser Kriterien zu einer eindeutigen Anknüpfung führt,

256 Es gibt zwar auch Verträge mit Verbraucherbeteiligung, die von Art. 6 Rom IVO nicht erfasst werden (v.a. im C2C Bereich), vgl. Leible in: NK-Rom VO, Art. 6 Rom I-VO Rn.31. Das ist hier aber nicht relevant, da der Provider stets Unternehmer ist und Nutzungsverträge im C2C-Bereich daher (fast) nicht vorkommen.

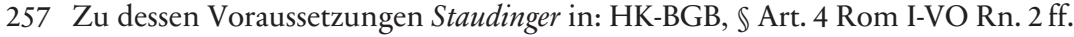


gilt nach Art. 4 Abs. 4 Rom I-VO das reine Prinzip der engsten Verbindung. ${ }^{258}$

In Betracht kommt zunächst, Nutzungsverträge als Dienstleistungsverträge im Sinne von Art. 4 Abs. 1 lit. b) Rom I-VO anzuknüpfen. Die vertragstypologische Bestimmung von Nutzungsverträgen nach deutschem Recht ist, wie bereits dargestellt, komplex und lässt sich nicht pauschal für alle Internetdienste einheitlich beantworten. ${ }^{259}$ Selbst wenn die Vertragstypen des Art. 4 Abs. 1 Rom I-VO autonom auszulegen sind und sich daher Unterschiede zwischen der Vertragstypologie nach nationalem Recht und der Einordnung von Verträgen in das System der Rom I-VO ergeben können, ${ }^{260}$ stellt die große Vielfalt der Internetdienste auch die Einordnung im Rahmen des Kollisionsrechts vor Herausforderungen. So ist der Dienstleistungsbegriff des Art. 4 Abs. 1 lit. b) Rom I-VO im unionalen Kollisionsrecht zwar weiter zu verstehen als der des deutschen Zivilrechts. ${ }^{261}$ Das wird beispielweise daran deutlich, dass der europäische Dienstleistungsbegriff auch Verträge im Sinne der $\$ \$ 631 \mathrm{ff}$. BGB erfasst. ${ }^{262}$ Gleichzeitig fallen aber Lizenzverträge über Immaterialgüterrechte nicht unter Art. 4 Abs. 1 lit. b) Rom I-VO. ${ }^{263}$ Lizenzrechtliche Aspekte werden indes in vielen Nutzungsverträgen (mit)geregelt, da Immaterialgüterrechte eine wichtige Rolle im Rahmen der Internetdienste spielen. ${ }^{264}$ Eine Unterscheidung zwischen Nutzungs- und Lizenzvertrag ließe sich nicht immer trennscharf aufrechterhalten. Beispielsweise im Rahmen von YouTube-Premium konvergieren Merkmale des sozialen Netzwerks mit Aspekten einer StreamingPlattform. Darüber hinaus gibt es parallel zum nationalen deutschen Recht eine kollisionsrechtliche Diskussion zum Einfluss der (Un-)Entgeltlichkeit auf die Vertragstypologie, die im Kontext der oftmals kostenfreien

258 Zur Normstruktur Leible in: NK-Rom VO, Art. 4 Rom I-VO Rn. 5; instruktiv Junker, Internationales Privatrecht, $\$ 15$ Rn. $21 \mathrm{ff}$.

259 Dazu bereits zuvor auf S. $33 \mathrm{ff}$.

260 Gläser, MMR 2015, 699, 700 ff.; Leible in: NK-Rom VO, Art. 4 Rom I-VO Rn. 17. Erwägungsgrund 17 Rom I-VO verweist für die Auslegung teilweise auf Art. 7 Nr. 1 lit. b) Brüssel Ia-VO.

261 Thorn in: Palandt, Art. 4 Rom I-VO Rn. 8; Martiny in: MüKo BGB, Art. 4 Rom IVO Rn. 34 ff.; Staudinger in: HK-BGB, $\$$ Art. 4 Rom I-VO Rn. 4.

262 Leible in: NK-Rom VO, Art. 4 Rom I-VO Rn. 29; Junker, Internationales Privatrecht, $\mathbb{1} 15$ Rn. 24.; Staudinger in: HK-BGB, $\$$ Art. 4 Rom I-VO Rn. 4.

263 Magnus in: Staudinger, Art. 4 Rom I-VO Rn. 40; Thorn in: Palandt, Art. 4 Rom IVO Rn. 8.

264 Das gilt für Lizenzen des Providers an Inhalten des Erblassers, s. dazu S. 194 ff., sowie für die umgekehrte Konstellation einer Lizenzierung von Medien an Nutzer. 
Internetdienste eine Einordnung nach Art. 4 Abs. 1 Rom I-VO zusätzlich erschwert. $^{265}$

Vor diesem Hintergrund bietet sich eine Lösung über die Auffangregel des Art. 4 Abs. 2 Rom I-VO an, der seinem Wortlaut nach auch dann zur Anwendung kommt, wenn „die Bestandteile des [anzuknüpfenden] Vertrags durch mehr als einen der Buchstaben [...] des Abs. 1 abgedeckt" sind. Das ist vorliegend sachgerecht etwa angesichts der Typenmischung aus Dienstleistung und Miete, Art. 4 Abs. 1 lit. b), c) und d) Rom I-VO, wie sie im Rahmen des Cloud-Computings und des Host-Providings vorliegen kann. ${ }^{266}$ In der Anwendung des Art. 4 Abs. 2 Rom I-VO besteht Einigkeit darüber, dass der Provider „die Partei [ist], welche die für den Vertrag charakteristische Leistung zu erbringen hat. “267 Die vom Provider geschuldete Informations- bzw. Kommunikationsleistung prägt den konkreten Nutzungsvertrag deutlich stärker als die schlichte Zahlung oder Datenpreisgabe durch den Nutzer. Damit kommt das Recht des gewöhnlichen Aufenthalts, also regelmäßig des realen Sitzes, ${ }^{268}$ des Providers zur Anwendung.

\section{b) Rechtswahlvereinbarung zwischen Unternehmern, Art. 3 Rom I-VO}

Haben die Parteien hingegen eine Rechtswahl getroffen, gelten die Vorgaben des Art. 3 Rom I-VO. Dessen Abs. 1 ermöglicht sowohl eine explizite als auch eine konkludente Wahl des anzuwendenden Rechts. Ob eine in AGB vorformulierte Rechtswahlklausel wirksam in die Einigung einbezogen wurde, ist eine Frage von „Zustandekommen und Wirksamkeit“, die sich nach dem Statut des Hauptvertrages bemisst, vgl. Art. 3 Abs. 5 in Ver-

265 Nach Leible in: NK-Rom VO, Art. 4 Rom I-VO Rn. 29 ist die Entgeltlichkeit eine zwingende Voraussetzung für den Dienstleistungsbegriff des Art. 4 Abs. 1 lit. b) Rom I-VO; a.A. aber Magnus in: Staudinger, Art. 4 Rom I-VO Rn. 40; dazu auch Gläser, MMR 2015, 699, 700 f. m.w.N. Zum Einfluss der Frage auf das nationale Recht und dort zu Data-for-Access-Konstellationen vgl. bereits zuvor auf S. $37 \mathrm{ff}$.

266 Kutscher, Der digitale Nachlass, S. 87 nennt hierfür E-Mail-Verträge als Beispiel; Nordmeier, MMR 2010, 151, dort Text in Fn. 13.

267 Leible in: NK-Rom VO, Art. 4 Rom I-VO Rn. 112; Gläser, MMR 2015, 699, 699 f.; Magnus in: Staudinger, Art. 4 Rom I-VO Rn. 608 und 621a jeweils m.w.N.; Kutscher, Der digitale Nachlass, S. 87 f.; Nordmeier, MMR 2010, 151, 152 lässt die Einordnung nach Art. 4 Abs. 1 Rom I-VO vor diesem Hintergrund ebenfalls explizit offen.

268 Magnus in: Staudinger, Art. 4 Rom I-VO Rn. 608; vgl. Art. 19 Rom I-VO. 
bindung mit Art. 10 Rom I-VO. ${ }^{269}$ Aus deutscher Perspektive gilt, wie stets, bei ausländischer Rechtswahl der Vorbehalt des ordre public, der sich hier aus Art. 21 Rom I-VO ergibt. ${ }^{270}$

\section{c) Zwischenergebnis}

Nutzungsverträge, die ohne Beteiligung von Verbrauchern geschlossen werden, unterliegen dem von den Parteien gewählten Recht. Wenn und soweit keine wirksame Rechtswahl getroffen wurde, unterliegt der Nutzungsvertrag dem Recht des Staates, in dem der Provider seine Hauptverwaltung hat, vgl. Art. 19 Abs. 1 S. 1 Rom I-VO, bzw. in dem sich die Niederlassung befindet, zu deren Vertrieb der Vertragsschluss gehört, vgl. Art. 19 Abs. 2 Rom I-VO. ${ }^{271}$

\section{Sonstige Erbrechtsobjekte und ihre Anknüpfung}

Neben vertraglichen Rechten und Pflichten können auch andere Rechtspositionen als Erbrechtsobjekte Bestandteil des digitalen Nachlasses sein. Dazu gehören insbesondere Urheberrechte bzw. urheberrechtliche Lizenzen des Erblassers sowie unter Umständen das Eigentum an beweglichen Sachen wie körperlichen Speichermedien.

Im Rahmen des urheberrechtlichen Kollisionsrechts ist aus der Perspektive des Erblassers beziehungsweise seiner Erben zu unterscheiden zwischen der Inhaberschaft an eigenen Urheberrechten und Lizenzen an Urheberrechten anderer. Letztere sind eng mit den soeben dargestellten Nutzungsverträgen verwandt und treten etwa im Kontext von Streaming-Plattformen oder bei privaten Mediatheken von erworbenen Musik- oder Filmdateien auf. Auf urheberrechtliche Lizenzen findet, wie auch auf Nutzungsverträge, das Kollisionsrecht für vertragliche Schuldverhältnisse und

269 Vgl. nur Junker, Internationales Privatrecht, $\mathbb{1} 15$ Rn. 12. Zur Rechtwahl qua AGB s. im Allgemeinen Magnus in: Staudinger, Art. 3 Rom I-VO Rn. 176 ff. und Stürner in: Erman, Art. 10 Rom I-VO Rn. 8 ff. sowie im Kontext des digitalen Nachlasses Budzikiewicz, AcP (218) 2018, 558, $588 \mathrm{f}$. und Kutscher, Der digitale Nachlass, S. $75 \mathrm{ff}$.

270 Magnus in: Staudinger, Art. 3 Rom I-VO Rn. 22. Art. 21 Rom I-VO geht Art. 6 EGBGB wegen des Vorrangs des Europarechts vor.

271 Für eine exemplarische Ermittlung der maßgeblichen Konzerngesellschaft für Facebook s. Gläser, MMR 2015, 699, 699 f. 
damit insbesondere die Rom I-VO Anwendung. ${ }^{272}$ Hierbei sind vor allem die Art. 3, 4 Abs. 2 und 6 Rom I-VO maßgeblich. ${ }^{273}$ Auf allfällige Rechtsstreite infolge einer (vermeintlichen) Urheberrechtsverletzung findet gem. Art. 8 Rom II-VO das Recht desjenigen Staates Anwendung, für den der Schutz beansprucht wird (sog. Schutzlandprinzip). In diesem Fall sind auch der Bestand und die Inhaberschaft eines Urheberrechts nach dem durch das Schutzlandprinzip bestimmten Recht zu ermitteln. ${ }^{274}$ Verletzungshandlungen, die online begangen werden, können in der Folge einen Strauß unterschiedlicher Rechtsordnungen zur Anwendung bringen und eine Mosaikbetrachtung notwendig machen, wenn und soweit der Urheberrechtsinhaber die Verletzung in mehreren Schutzländern verfolgt. ${ }^{275}$ Das gilt folglich auch für Urheberrechte, die ursprünglich dem Erblasser zustanden und als Teil des digitalen Nachlasses auf die Erben übergegangen sein könnten. ${ }^{276}$

Das auf das Eigentum an Speichermedien anzuwendende Sachstatut wird maßgeblich durch die Art. 43 und 46 EGBGB bestimmt. ${ }^{277}$ Art. 43 Abs. 1 EGBGB unterwirft subjektive dingliche Rechte an einer Sache dem

272 Stimmel, GRUR Int. 2010, 783, 783 ff.; Martiny in: MüKo BGB, Art. 4 Rom I-VO Rn. 244.; Wandtke, Urheberrecht, Kap. 10 Rn. 6.

273 Insoweit kann im Wesentlichen auf die Ausführungen zur Anknüpfung von Nutzungsverträgen auf S. $73 \mathrm{ff}$. verwiesen werden. Vgl. auch Fezer/Koos in: Staudinger, Internationales Wirtschaftsrecht, Rn. 1123.

274 So die st. Rspr., vgl. BGH GRUR 2018, 178 Rn. 13 - Vorschaubilder III; BGH GRUR Int. 2015, 375 Rn. 24 - Hi Hotel II; BGH GRUR 2016, 490 Rn. 24 - Marcel-Breuer-Möbel II; Wandtke, Urheberrecht, Kap. 10 Rn. 7; Fezer/Koos in: Staudinger, Internationales Wirtschaftsrecht, Rn. 1072 ff.; a.A. Schack, Urheberund Urhebervertragsrecht, Rn. $1011 \mathrm{ff}$. und insbesondere 1019ff.; Drexl in: MüKo BGB, Internationales Wirtschaftsrecht, Teil 8. Internationales Immaterialgüterrecht, Rn. $175 \mathrm{ff}$.

275 Kutscher, Der digitale Nachlass, S. 69; Grünberger in: NK-Rom VO, Art. 8 Rom II-VO Rn. 44; ausführlich Drexl in: MüKo BGB, Internationales Wirtschaftsrecht, Teil 8. Internationales Immaterialgüterrecht, Rn. $296 \mathrm{ff}$. sowie $\mathrm{Fe}$ zer/Koos in: Staudinger, Internationales Wirtschaftsrecht, Rn. 1072, $1107 \mathrm{ff}$.

$276 \mathrm{Zu}$ Beispielen möglicher Urheberrechte des Erblassers vgl. noch auf S. $188 \mathrm{ff}$. Das Immaterialgüterstatut genießt Vorrang gegenüber dem Erbstatut, soweit es vom allgemeinen Erbrecht abweichende Regelungen enthält, wie etwa $\$ 28$ Abs. 2 S. 2 UrhG. Denn die inhaltliche Ausgestaltung des Immaterialgüterrechts ist untrennbar mit dem Immaterialgüterstatut verbunden und sollte daher auch dessen Recht unterliegen, vgl. dazu Dutta in: MüKo BGB, Art. 1 EuErbVO Rn. 54; Schmidt in: Dutta/Weber (Hrsg.), Internationales Erbrecht, Art. 1 EuErbVO Rn. 146.

277 Allgemein zum internationalen Sachenrecht $v$. Bar/Mankowski, Internationales Privatrecht II, $\$ 3$ passim; Rauscher, Internationales Privatrecht, Rn. $1529 \mathrm{ff}$. 
Recht des Belegenheitsorts der betroffenen Sache. ${ }^{278}$ Beispielsweise die Vererbbarkeit einer externen Festplatte, die sich bei den persönlichen Gegenständen des Erblassers in seinem Haus in Deutschland befindet, richtet sich mithin nach deutschem Sachenrecht. Art. 46 EGBGB enthält demgegenüber eine Ausweichklausel für Sachverhalte, in denen zu einer anderen als der nach Art. 43 EGBGB berufenen Rechtsordnung eine wesentlich engere Verbindung besteht. ${ }^{279}$ Die Möglichkeit einer privatautonomen Rechtswahl eröffnet der nationale deutsche Gesetzgeber mit Blick auf die Ordnungsinteressen des Rechtsverkehrs nicht. ${ }^{280}$

\section{Zusammenfassung}

Es lässt sich keine allgemeingültige, übergreifende Anknüpfung für Rechtsfragen des digitalen Nachlasses benennen. Vielmehr ist nach dem jeweils infrage stehenden Erbrechtsobjekt zu unterscheiden und sodann einzeln anzuknüpfen. Der digitale Nachlass ist insofern nicht internationalprivatrechtlich verklammert. Im Gegenteil wirkt seine Vielfalt in das Kollisionsrecht fort, wodurch eine einheitliche Antwort auf die Frage nach dem anwendbaren Recht in allfälligen Rechtsstreiten im Kontext des digitalen Nachlasses nicht möglich ist.

Aus der bereits zuvor konstatierten „Verschuldrechtlichung [...] der Universalsukzession 2.0"281 folgt hier die besondere Bedeutung des Vertragsstatuts und der Rom I-VO. ${ }^{282}$ Denn den Nutzungsverträgen kommt als Erbrechtsobjekten im digitalen Nachlass, wie gesehen, eine hervorgehobene Bedeutung zu. Diese Nutzungsverträge sind vielfach Verbraucherverträge und daher nach den Sonderregeln des Art. 6 Abs. 1 und 2 Rom I-VO anzuknüpfen. In den Fällen, in denen kein Verbraucher an dem Vertrags-

278 Sog. lex rei sitae bzw. lex situs, vgl. v. Bar/Mankowski, Internationales Privatrecht II, $\$ 3$ Rn. 12; Rauscher, Internationales Privatrecht, Rn. $1530 \mathrm{f}$.

279 Dazu $v$. Bar/Mankowski, Internationales Privatrecht II, $\mathbb{} 3$ Rn. 24 ff.; zu einzelnen Fallgruppen vgl. Wendehorst in: MüKo BGB, Art. 46 EGBGB Rn. 30 ff.

280 Weiterführend zu der (umstrittenen) Frage, ob sich aus europäischem Recht ein anderes Ergebnis ableiten lässt $v$. Bar/Mankowski, Internationales Privatrecht II, \3 Rn. 169 ff.; Wendehorst in: MüKo BGB, Art. 46 EGBGB Rn. 18 ff. m.w.N.

281 Sorge, MMR 2018, 372, 377. Dazu zuvor im Kontext der Diskussion zu einem Recht an Daten auf S. 68 f.

282 Im Bericht der Arbeitsgruppe „Digitaler Neustart“ der Konferenz der Justizministerinnen und Justizminister der Länder, S. 403 f., abrufbar a.a.O. (Fn. 43), wird auch ausschließlich des IPR bezüglich „vertraglicher Regelungen“ thematisiert. 
verhältnis beteiligt ist, kommt hingegen der Rechtswahl nach Art. 3 Rom I-VO und der objektiven Grundanknüpfung nach Art. 4 Rom I-VO entscheidende Bedeutung zu.

\section{B. Nutzungsverträge als Erbrechtsobjekt}

Die Vererbbarkeit eines Nutzerkontos in sozialen Medien war der Gegenstand des bislang einzigen höchstrichterlichen Urteils im Bereich des digitalen Nachlasses. ${ }^{283}$ Konkret ging es in dem Sachverhalt um die Frage, ob die Erben der minderjährigen Inhaberin eines Facebook-Accounts qua Universalsukzession in den Nutzungsvertrag zwischen Erblasserin und Facebook einrücken. ${ }^{284}$ An dieser Konstellation entfaltet sich auf exemplarische Weise das Geflecht der widerstreitenden Interessen aller Beteiligten - also vor allem der Parteien des Nutzungsvertrags, der Erben und der Kommunikationspartner des Erblassers. Für die jeweiligen Interessen lassen sich unterschiedliche Regelungsregime in Ansatz bringen, welche sich zum einen auf die grundsätzliche Übergangsfähigkeit des Nutzungsvertrags im Erbgang (dazu II.) und zum anderen auf die Durchsetzbarkeit der übergegangenen Rechtsposition (dazu III.) auswirken können. Daraus ergibt sich das gesetzlich vorgesehene Ergebnis zu Nutzungsverträgen im Erblass. Fraglich ist sodann, ob und inwieweit den Parteien ein (formular-)vertraglicher Gestaltungsspielraum verbleibt, in dessen Wahrnehmung sie von dem gesetzlichen Ergebnis abweichen können (dazu IV.). Alledem ist eine allgemeine Einführung zu Verträgen als Erbrechtsobjekten voranzustellen (dazu I.).

283 BGH NJW 2018, 3178. Ein neueres Urteil des LG Münster ErbR 2019, 455 betrifft die Vererbbarkeit eines iCloud-Accounts. Es handelt sich dabei um ein Versäumnisurteil, in dessen Begründung lediglich kurz auf das o.g. FacebookUrteil des BGH verwiesen wird.

284 Zur Folgefrage der Zwangsvollstreckung des aus dem Nutzungsvertrag folgenden Rechts auf Zugang zu den Accountinhalten, vgl. Seidler, NZFam 2020, 141, 143 f. sowie BGH ZEV 2020, 714 mit kritischer Anm. v. Elzer, FD-ZVR 2020, 432500 und mit zustimmender Anm. v. Lieder/Berneith, FamRZ 2020, 1761, $1762 \mathrm{f}$. Die Instanzen hatten sehr unterschiedlich argumentiert und entschieden, vgl. LG Berlin ZUM-RD 2019, 613 und KG Berlin ZEV 2020, 176 mit Anm. v. Deusch ebd. 
I. Einführung: Vertragliche Rechtsverhältnisse als Erbrechtsobjekte

Die Betrachtung des Nutzungsvertrags beginnt mit der grundsätzlichen Frage danach, wie sich der Tod einer Vertragspartei auf das Schicksal eines Vertrages auswirkt. Nach $\$ 1922$ Abs. 1 BGB geht das Vermögen des Erblassers mit dem Erbfall im Wege der Universalsukzession insgesamt und ungeteilt „als Ganzes“ ipso iure auf den (oder die) Erben über. ${ }^{285}$ Dazu gehören Ansprüche und Verbindlichkeiten aus schuldrechtlichen Verträgen. ${ }^{286}$ Der Erbe rückt in das Rechtsverhältnis - also umfassend in die gesamte rechtliche Beziehung - zwischen einer Person und dem Erblasser ein. ${ }^{287}$ Das schließt neben sämtlichen vertraglichen Rechten und Pflichten auch Hilfsansprüche und Gestaltungsrechte ein. ${ }^{288}$ Der gesetzliche Nachfolgemechanismus bewirkt demnach, dass der Erbe nach dem Erbgang in der vertraglichen Stellung des Erblassers steht. Im Grundsatz sind vertragliche Rechtsverhältnisse somit vererbbar.

Dieser Grundsatz gilt indes nicht ausnahmslos. Zunächst können die Parteien in der privatautonomen Ordnung des BGB selbst entscheiden, ob ihre vertragliche Beziehung einer Rechtsnachfolge unterworfen sein soll. Denn aus der Vertragsfreiheit folgt, dass die Parteien den Übergang vertraglicher Rechte und Pflichten im Erbfall beschränken oder ausschließen können. ${ }^{289}$ Dies ist innerhalb der weiten Grenzen der $\$ \$ 138,242,313$ BGB jedenfalls im Wege einer Individualabrede ohne Weiteres möglich. Etwas anderes kann gelten, wenn die Vererbbarkeit mittels AGB ausgeschlossen werden soll. Entsprechende Klauseln obliegen der Einbeziehungs- und Inhaltskontrolle der $\$ \$ 305 \mathrm{ff}$. BGB. ${ }^{290}$ Ferner bestehen vom Grundsatz der Vererbbarkeit auch gesetzliche Ausnahmen. Schuldrechtsimmanent ord-

285 Weidlich in: Palandt, $\mathbb{1} 1922$ BGB Rn. 10.

286 Lange, Erbrecht, $\mathbb{9}$ Rn. 32; Hoeren in: HK-BGB, $\mathbb{} 1922$ BGB Rn. 12; Alexander, K\&R 2016, 301, 306; Dietzel, Untergang statt Fortbestand, passim.

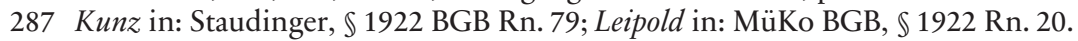

288 Lange, Erbrecht, $\$ 9$ Rn. 32.

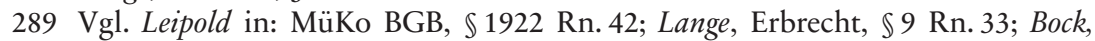
AcP (217) 2017, 370, 372; BGH NJW 2018, 3178 Rn. 24. Dieser Grundsatz gilt freilich nicht unbeschränkt. Hingewiesen sei etwa auf $\$ 563 \mathrm{BGB}$, der für Wohnraummietverträge im Falle des Todes des Mieters für einen bestimmten Kreis Anspruchsberechtigter ein Eintrittsrecht in den Mietvertrag vorsieht. Dieses Recht kann von den ursprünglichen Mietvertragsparteien nicht abbedungen werden, $\$ 563$ Abs. 5 BGB.

290 BGH NJW 2018, 3178 Rn. 28 ff. prüft obiter die Wirksamkeit einer entsprechenden Klausel im Facebook-Nutzungsvertrag. Der BGH stellt hier Verstöße gegen $\$ 307$ Abs. 2 Nr. 1 und 2 BGB fest. Siehe zum Ganzen auf S. 162 ff. 
net das BGB beispielsweise für manche Vertragsarten, vgl. $\$ \$ \$ 613,673$ S. 1, 675 Abs. 1 BGB, sowie für manche Vertragsgegenstände, vgl. $\$ 520$ BGB, an, dass die vertraglichen Beziehungen mit dem Tode eines Vertragspartners enden sollen. ${ }^{291}$ Parallel dazu kann sich die Unvererbbarkeit aus der Höchstpersönlichkeit einer vertraglichen Beziehung ergeben. ${ }^{292}$

Sofern die Parteien - wie regelmäßig - keine explizite Vereinbarung getroffen haben und außerdem keine (gesetzliche) Ausnahme einschlägig ist, kommt indes die erbrechtliche Grundregel zur Anwendung, nach der vertragliche Rechtsverhältnisse vollumfänglich der Rechtsnachfolge von Todes wegen unterfallen.

\section{Höchstpersönlichkeit als die Grenze der Vererbbarkeit}

Die Inhalte eines Social Media Accounts, zu denen öffentlich einsehbare Posts sowie Privatnachrichten gehören, können in ihrer Gesamtheit ein sehr genaues, digitales Abbild des Nutzers darstellen. Entsprechend sind bei solchen Accounts neben der Sozial- auch die Privatsphäre und mit der Intimsphäre selbst der Kernbereich des Persönlichkeitsrechts betroffen, beispielsweise wenn der Nutzer im privaten Chat gegenüber Dritten vertrauliche Informationen preisgibt. ${ }^{293}$ Das ist nicht zwangsläufig der Fall, wie ein Blick auf geschäftlich genutzte Accounts zeigt, kommt aber häufig vor. An dem Informationsgefüge sind mit dem Nutzer, seinen Kommunikationspartnern und dem Provider zunächst drei Parteien beteiligt. Durch den Erbgang kommen die Erben als eine neue, vierte Partei hinzu, die - sofern und soweit der Nutzungsvertrag vererbbar ist - umfassend Einblick in die Accountinhalte erhält.

291 Lange, Erbrecht, $\mathbb{\$} 9$ Rn. 32; Kunz in: Staudinger, $\mathbb{} 1922$ BGB Rn. 469 ff.; Hoeren in: HK-BGB, $\mathbb{S} 1922$ BGB Rn. 12. Das schließt nicht aus, dass aus der Abwicklung des Schuldverhältnisses noch einzelne Ansprüche der Erben (z.B. der Anspruch auf bereits verdiente Dienstvergütung) oder des Vertragspartners (z.B. Herausgabeansprüche) resultieren, vgl. Müller-Glöge in: MüKo BGB, $\$ 613$ Rn. $10 \mathrm{ff}$.

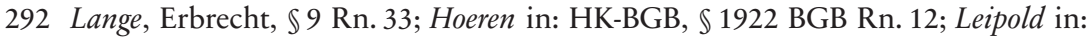
MüKo BGB, $\mathbb{1} 1922$ Rn. 26 legt als Maßstab $\$ 399$ BGB analog an.

293 Das Gutachten der Datenethikkommission vom 23.10.2019, S. 111, nennt hier als Beispiel das „Online-Konto in einer Gruppe ,Anonymer Alkoholiker““, abrufbar a.a.O. (Fn. 30). 
Vor diesem Hintergrund kann die Höchstpersönlichkeit, die eine Grenze der Vererbbarkeit darstellt, ${ }^{294}$ in zwei unterschiedlichen Konstellationen Bedeutung erlangen: Bei Nutzungsverträgen über Soziale Medien steht zuerst das Gefüge zwischen Provider, Erblasser und den Kommunikationspartnern des Erblassers im Vordergrund (dazu 1.). Die Berücksichtigung der Interessen des Providers und des Erblassers ergibt sich insofern schon aus deren Stellung als Parteien des Nutzungsvertrags. In diesem Rahmen sind auch die Interessen der Kommunikationspartner des Erblassers zu berücksichtigen, die bei der Nutzung des Accounts sowie bei dessen Übergang von Erblasser auf Erbe zwangsläufig ebenfalls betroffen werden. Als zweiter Anwendungsfall sehen Teile des Schrifttums die Höchstpersönlichkeit der Accountinhalte im Verhältnis zwischen dem Erblasser und seinen Erben als Hindernis für die Vererbbarkeit des Nutzungsvertrags (dazu 2.). Hier treten die nächsten Angehörigen des Erblassers als die Wahrnehmungsberechtigten des postmortalen Persönlichkeitsrechts des Verstorbenen als weitere Partei hinzu.

1. Höchstpersönlichkeit im Gefüge zwischen Provider, Erblasser und Kommunikationspartnern des Erblassers

Der Begriff der Höchstpersönlichkeit ist im Erbrecht nicht eigens explizit geregelt und birgt aufgrund seiner Unbestimmtheit eine gewisse Unschärfe. Als Rahmen und als Maßstab zur Bestimmung der Unterscheidung zwischen regulär vererbbaren Rechtsverhältnissen (der Regelfall) und solchen Rechtsverhältnissen, die aufgrund von Höchstpersönlichkeit unvererbbar sind (die Ausnahme), hat sich im vertraglichen Bereich die analoge Anwendung des $\$ 399$ Fall 1 BGB durchgesetzt. ${ }^{295}$ Im Folgenden wird diese Norm zunächst in ihrem originären Regelungskontext dargestellt, bevor ihr Gehalt sodann auf den digitalen Nachlass bezogen wird.

294 Vgl. Bock, AcP (217) 2017, 370, 382 ff. sowie bereits die Nachweise in Fn. 292.

295 Vgl. nur BGH NJW 2018, 3178 Rn. 34 m.w.N. Der BGH nennt zusätzlich den Rechtsgedanken des $\$ 38$ BGB; s. ferner Leipold in: MüKo BGB, $\$ 1922$ Rn. 26. 
a) Der Rechtsgedanke des $₫ 399$ Fall 1 BGB im Erbrecht allgemein

Dem Grundsatz der freien Übertragbarkeit von Forderungen im Wege der Abtretung setzt $\ 399$ Fall 1 BGB eine Ausnahme entgegen. ${ }^{296}$ Danach kann eine Forderung „nicht abgetreten werden, wenn die Leistung an einen anderen als den ursprünglichen Gläubiger nicht ohne Veränderung ihres Inhalts erfolgen kann [...]. “ Bei der Anwendung von $\$ 399$ Fall 1 BGB werden mehrere Fallgruppen unterschieden. ${ }^{297}$ Von Interesse sind vorliegend vornehmlich Fälle der Änderung des Leistungsinhalts durch Abtretung sowie der personengebundenen Ansprüche, für die sich die Unabtretbarkeit daraus ergibt, dass die Person des Gläubigers für den Inhalt des Anspruchs wesentlich ist. ${ }^{298} \mathrm{Zu}$ diesen höchstpersönlichen, zessionsfeindlichen Ansprüchen gehören beispielsweise der Anspruch auf Urlaub(sabgeltung), der Anspruch auf Unterlassung einer Persönlichkeitsrechtsverletzung oder der Anspruch auf Pflegeleistungen. ${ }^{299}$

Der skizzierte Rechtsgedanke des $₫ 399$ Fall 1 BGB hat Einzug in das Erbrecht gefunden. ${ }^{300}$ Nicht nur entspricht das Regel-Ausnahme-Verhältnis von abtretungsfähigen und abtretungsfeindlichen Ansprüchen demjenigen von vererbbaren und unvererbbaren Rechtsverhältnissen, sondern auch muss angesichts des Subjektwechsels von Zedenten zu Zessionar beziehungsweise von Erblasser zu Erbe eine ähnliche Konfliktlage austariert werden. In Anlehnung an den Rechtsgedanken des $\$ 399$ Fall 1 BGB ist eine Forderung also dann nicht vererblich, wenn sie in einem solchen Maße auf die Person des Schuldners oder des Gläubigers zugeschnitten ist, dass die versprochene Leistung durch eine Rechtsnachfolge in ihrem Wesen verändert würde. ${ }^{301}$ In der Literatur wird hier regelmäßig das Beispiel

296 S. Busche in: Staudinger, $\$ 399$ BGB Rn. 1 ff. zu dieser und weiteren Ausnahmen; Grüneberg in: Palandt, $\$ 399$ BGB Rn. 1.

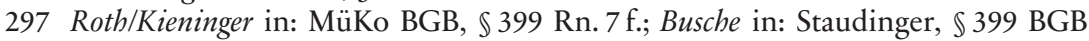

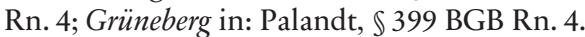

298 Rotb/Kieninger in: MüKo BGB, $\$ 399$ Rn. 7 und 9 ff.; Grüneberg in: Palandt, $\$ 399$ BGB Rn. $4 \mathrm{ff}$.

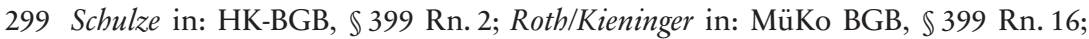
zahlreiche Beispiele finden sich bei Busche in: Staudinger, $\mathbb{3} 399$ BGB Rn. 7 ff.; Grüneberg in: Palandt, $\$ 399$ BGB Rn. 4 ff.

300 Leipold in: MüKo BGB, $\$ 1922$ Rn. 26; BGH NJW 2018, 3178 Rn. 34; kritisch dazu Fußeder, Soziale Netzwerke im Nachlass, S. 77 f.

301 Lange, Erbrecht, $\mathbb{9}$ Rn. 33; Leipold in: MüKo BGB, $\mathbb{} 1922$ Rn. 26; Hoeren in: HK-BGB, $\$ 1922$ BGB Rn. 12. 
eines Künstlers bemüht, dessen Erben nicht verpflichtet sein können, seine Auftragswerke anzufertigen oder abzuschließen. ${ }^{302}$

b) Der Rechtsgedanke des $₫ 399$ Fall 1 BGB im digitalen Nachlass

Bezieht man den Rechtsgedanken des $\$ 399$ Fall 1 BGB auf den Nutzungsvertrag zwischen Provider und Erblasser, ist nach den jeweiligen Pflichten der Parteien zu differenzieren. Diese Pflichten können sich zwischen den verschiedenen Internetdiensten zwar durchaus unterscheiden. Im Wesentlichen wird aber der Provider verpflichtet sein, eine IT-Infrastruktur zu unterhalten und dem Nutzer dazu mittels eines Accounts Zugang zu gewähren. Der Nutzer stellt seine persönlichen Daten zur Verfügung und setzt sich dadurch während der Nutzung personalisierter Werbung aus. ${ }^{303}$

\section{aa) Beurteilung der Pflichten des Providers}

In seiner Beurteilung der providerseitigen Pflichten am Maßstab des Rechtsgedankens aus $\$ 399$ Fall 1 BGB nimmt der BGH eine formal-technische Betrachtungsweise vor. Das Gericht trennt zwischen den (vermeintlich) rein technischen Leistungspflichten des Providers, die für jeden Nutzer gleich und daher nicht persönlichkeitsrechtlich relevant seien, und den Accountinhalten wie der Gestaltung der Profilseite, Fotos und Privatnachrichten, die von den Nutzern erstellt werden und persönlichkeitsrechtliche Bezüge aufweisen können. ${ }^{304}$ Die technische Leistung des Providers - insbesondere die Übermittlung von Inhalten und Nachrichten - könne unverändert auch gegenüber den Erben erbracht werden. ${ }^{305}$ Zuvor hat bereits Seidler argumentiert, dass zwischen Persönlichkeitswerten und ihrer Ver-

302 Vgl. nur Leipold in: MüKo BGB, $\mathbb{S} 1922$ Rn. 26, der auch keine Pflicht des Privatlehrers zum Unterricht der Erben seines verstorbenen Schülers sieht; vgl. auch Lange, Erbrecht, $\$ 9$ Rn. 33.

303 Zu den Pflichten des Providers s. S. $91 \mathrm{f}$. und zu denen des Nutzers s. S. 93 f.

304 BGH NJW 2018, 3178 Rn. 35; so auch schon Kutscher, Der digitale Nachlass, S. 156 f.; a.A. Klas/Möhrke-Sobolewski, NJW 2015, 3473, 3474.

305 BGH a.a.O. (Fn. 304); Herzog/Pruns, Der digitale Nachlass in der Vorsorge- und Erbrechtspraxis, S. 78; Bericht der Arbeitsgruppe „Digitaler Neustart“ der Konferenz der Justizministerinnen und Justizminister der Länder, S. 328, abrufbar a.a.O. (Fn. 43). 
körperung zu unterscheiden sei. ${ }^{306}$ Als Beispiel nennt sie den Inhalt eines Tagebuchs, der zwar postmortalen Persönlichkeitsrechtsschutz genieße, ohne dass daraus aber die Höchstpersönlichkeit des Sacheigentums an dem Papier des Tagebuchs folge. Parallel seien zwar die Kontoinhalte, nicht jedoch der Nutzungsvertrag höchstpersönlich. ${ }^{307}$

Leipold hingegen will darauf abstellen, ob das gesamte Vertragsverhältnis entscheidend durch höchstpersönliche Zwecke des Nutzers geprägt ist. $^{308}$ Die Argumentation des BGH nennt Leipold „etwas formal. “" ${ }^{09}$ Dem ist zuzustimmen. Denn eine Gesamtbetrachtung des Vertragsverhältnisses erlaubt einen flexibleren Umgang mit den unterschiedlichen Internetdiensten, die schon heute existieren und deren weitere Entwicklung nicht vorherzusehen ist. Die rein formal-technische Ansicht des BGH ließe keine Unterscheidung zwischen einem beruflich genutzten Netzwerk wie LinkedIn, einem vorrangig privaten Zwecken dienendem Netzwerk wie Facebook und Dating-Apps zu, die intimsten Interessen dienen. ${ }^{310}$ Solange die Vererbbarkeit eines Vertragsverhältnisses, wie gesetzlich vorgesehen, der Regelfall bleibt, können mittels Gesamtbetrachtung notwendige Ausnahmen benannt werden. Hier hat das Kriterium des $\$ 399$ Fall 1 BGB gerade den Vorteil, dass es unabhängig von der Unterscheidung zwischen analogem und digitalem Erbe sachgerecht einen Gleichlauf von (vergleichbaren) analogen und digitalen Sachverhalten ermöglicht. ${ }^{311}$ Wenn man davon ausgeht, dass Plattform- und Nutzungsverträge in der Zukunft einen immer größeren Teil des bisher analogen Lebens digital abbilden werden,

306 Seider, Digitaler Nachlass, S. 136; ausführlich wiedergegeben bei Bericht der Arbeitsgruppe „Digitaler Neustart“ der Konferenz der Justizministerinnen und Justizminister der Länder, S. 327-329, abrufbar a.a.O. (Fn. 43).

307 Seidler, Digitaler Nachlass, S. 136; ebenfalls mit einem Vergleich zur analogen Welt argumentieren Herzog/Pruns, Der digitale Nachlass in der Vorsorge- und Erbrechtspraxis, S. 77 f.: Ein Nutzungskonto in einem sozialen Netzwerk unterscheide sich von der Mitgliedschaft in einem Verein, die gem. $₫ 38$ S. 1 BGB im Grundsatz weder übertragbar noch vererblich ist, dadurch, dass bei Abschluss des Nutzungsvertrags - anders als bei der Aufnahme in den Verein - die Identität des Erblassers keine Rolle spiele; dem folgend Willems, ZfPW 2016, 494, 506 f.; vgl. ferner Raude, RNotZ 2017, 17, 20.

308 Leipold in: MüKo BGB, $\mathbb{1} 1922$ Rn. 33.

309 Im Ergebnis aber trotzdem wie BGH so Leipold in: MüKo BGB, $\mathbb{1} 1922$ Rn. 33.

310 Mit anderer Argumentation für eine Ausnahme bei (online) Partnerschaftsbörsen ist auch Budzikiewicz, AcP (218) 2018, 558, 569 f.; Bericht der Arbeitsgruppe „Digitaler Neustart" der Konferenz der Justizministerinnen und Justizminister der Länder, S. 340, abrufbar a.a.O. (Fn. 43).

311 Bericht der Arbeitsgruppe „Digitaler Neustart“ der Konferenz der Justizministerinnen und Justizminister der Länder, S. 338, abrufbar a.a.O. (Fn. 43). 
dürfte es nicht sinnvoll sein, das erarbeitete Unterscheidungskriterium durch eine pauschale formal-technische Betrachtungsweise faktisch aufzugeben.

\section{bb) Beurteilung der Pflichten des Nutzers}

In der Diskussion haben die nutzerseitigen Pflichten bislang weniger Aufmerksamkeit erfahren als die Providerpflichten. So stellt der BGH zentral darauf ab, dass kein schutzwürdiges Interesse des Providers daran bestehe, seine Leistung nicht auch gegenüber den Erben erbringen zu müssen. ${ }^{312}$ Wenn und soweit die Erben aber über den schlichten Zugang hinaus das Recht zur fortgesetzten Nutzung des Erblasseraccounts begehren, ${ }^{313}$ können auch die Leistungen des Nutzers einen besonderen Personenbezug aufweisen. ${ }^{314}$ Denn die Internetdienste basieren oftmals auf Dienste-gegenDaten Modellen. ${ }^{315}$ Die Wertschöpfung aufseiten der Provider basiert darauf, präzise Werbeprofile der Nutzer anzulegen und damit Werbekunden zu akquirieren. Ein Subjektwechsel aufseiten des Nutzers würde - zumindest bei einer aktiven Weiternutzung des Accounts durch den neuen Inhaber - zu einer Vermischung der Nutzungsdaten und damit zu einem nicht mehr eindeutigen Werbeprofil führen. ${ }^{316}$ Das wiederum schmälert die Attraktivität des Angebots der Provider gegenüber den Werbekunden erheblich, da der in der Präzision liegende Vorteil vor herkömmlicher, breiten(un)wirksamer Werbung verloren geht.

Dieses Problem stellt sich hingegen nicht für werbefreie Premiumversionen, die dafür oftmals kostenpflichtig sind, oder für sonstige Internetdienste, die Daten nicht in der beschriebenen Form nutzen. ${ }^{317}$ Provider

312 BGH NJW 2018, 3178 Rn. 36 a.E.

313 Zur Frage nach der Reichweite des Erbrechts vgl. noch auf S. $100 \mathrm{ff}$.

314 Dazu bislang hauptsächlich Budzikiewicz, AcP (218) 2018, 558, 570 f.; Gomille, ZUM 2018, 660, 666 sieht ein berechtigtes Interesse des Providers „an der Vermeidung einer Zuordnungsverwirrung“.

$315 \mathrm{Zu}$ mehrseitiger Finanzierung in Data-for-Access-Konstellationen vgl. schon zuvor auf S. $37 \mathrm{ff}$.

316 Auch Seidler, Digitaler Nachlass, S. 136 warnt davor, dass sich Nutzungshandlungen des Erblassers und der Erben in einem Account mischen können, bezieht dies jedoch nicht auf $\$ 399$ Fall 1 BGB und die Data-for-Access-Konstellation.

317 Budzikiewicz, AcP (218) 2018, 558, 570 f. folgert daraus, dass eine Fortführung von Social-Media-Accounts durch die Erben, anders als bei E-Mail-Diensten, regelmäßig ausgeschlossen sei. 
schließen Verträge im Internet regelmäßig ohne genaue Prüfung der Identität und Solvenz ihrer Vertragspartner (i.e. der Nutzer), sodass ein Vertragspartnerwechsel im Wege der Universalsukzession für die Provider unproblematisch erscheint, wenn die Gegenleistung des Nutzers (ausschließlich) eine Geldzahlung ist. ${ }^{318}$

\section{cc) Zusammenfassung}

Die Vielfalt der Internetdienste verbietet eine pauschale Beurteilung der Vererbbarkeit unter dem Gesichtspunkt des $\$ 399$ Fall 1 BGB. Zwar bleibt die Vererbbarkeit von vertraglichen Rechtsverhältnissen auch für Nutzungsverträge der gesetzlich vorgesehene Regelfall. Gleichwohl setzt die Vielfalt der Providerangebote mit ihrem unterschiedlichen Maß an Persönlichkeitsbezug und die ebenso diverse Ausgestaltung der nutzerseitigen Pflichten aber eine genaue Betrachtung des konkret infrage stehenden Vertragsverhältnisses voraus. Es ist im Wege einer Gesamtbetrachtung zu prüfen, ob das Vertragsverhältnis durch höchstpersönliche Zwecke geprägt ist.

\section{c) Vertragsimmanenter Schutz der Kommunikationspartner}

Die Frage, ob und inwieweit die Interessen der Kommunikationspartner des Erblassers der Vererbbarkeit selbst oder zumindest ihrer Durchsetzbarkeit entgegensteht, wird im Zusammenhang mit mehreren Regelungsmaterien relevant. Der BGH geht dieser Frage gleich an drei Stellen nach, indem er die Interessen von Dritten und Kommunikationspartnern auf vertraglicher, telekommunikations- und auf datenschutzrechtlicher Ebene berücksichtigt. ${ }^{319}$ Die jeweils vorgetragenen Argumente und Wertungen ähneln einander und fußen letztlich allesamt auf Aspekten des Persönlichkeitsrechts der Kommunikationspartner des Erblassers. ${ }^{320}$ Gleichwohl sind die Regime dogmatisch voneinander getrennt zu würdigen. Denn die Regelungsmaterien unterscheiden sich sowohl in ihren Tatbestandsvoraussetzungen als auch in ihren Rechtsfolgen.

Auf vertraglicher Ebene prüft der BGH im Rahmen und am Maßstab von $\$ 399$ BGB analog, ob „der höchstpersönliche Charakter und damit

318 Bock, AcP (217) 2017, 370, 395 f.

319 BGH NJW 2018, 3178 Rn. 39 ff., 54 ff., 88 ff.

320 „Überschneidungen“ konstatiert auch Pruns, ErbR 2018, 614, 618. 
der vertragliche Ausschluss der Vererbbarkeit des Zugangsrechts zu dem Benutzerkonto [...] aus im Nutzungsvertrag stillschweigend vorausgesetzten und damit immanenten Gründen des Schutzes der Persönlichkeitsrechte der Kommunikationspartner" folgt. ${ }^{321}$ Der BGH führt sodann im Wesentlichen drei Argumente gegen das Vorliegen von Höchstpersönlichkeit an: Erstens sieht er nur eine konto- und keine personenbezogene Pflicht des Providers bei der Zustellung elektronischer Nachrichten (dazu aa). Weiterhin argumentiert das Gericht mit der (vermeintlichen) Erwartungshaltung eines verständigen Nutzers (dazu bb). Schließlich analysiert der BGH die mangelnde Verfügungsbefugnis des Nutzers über eine Nachricht nach deren Versenden (dazu cc).

\section{aa) Konto- oder personenbezogene Pflicht des Providers}

Eine zentrale Bedeutung für den BGH hat ein Vergleich zur analogen Briefpost, aus dem er folgert, den Provider treffe nur eine konto-, aber keine personenbezogene Pflicht bei der Vermittlung von Kommunikationsinhalten. ${ }^{322}$ Eine elektronische Nachricht werde ebenso wie ein Brief (nur) an ein Nutzerkonto bzw. einen Briefkasten und nicht an eine bestimmte Person zugestellt. ${ }^{323}$ Sei die Zustellungspflicht des Providers aber schon nicht personen-, sondern nur kontobezogen, dann könne sie nicht höchstpersönlich sein.

So sehr dieser Vergleich sich prima facie aufdrängen mag, so sehr hinkt er bei näherer Betrachtung jedoch auch. Die Gegenüberstellung übersieht, dass die technischen Möglichkeiten und das Nutzungsverhalten inhaltlich wie funktional eine andere Dimension erreicht haben als ein herkömmlicher Briefwechsel. ${ }^{324}$ Wer Zugang zu sämtlichen Nutzungskonten des Erb-

321 BGH NJW 2018, 3178 Rn. 39. Das Gericht verneint dies im Ergebnis freilich.

322 BGH NJW 2018, 3178 Rn. 41 mit Verweis auf Herzog, ZErb 2017, 205, 208; dies. in: NK-Nachfolgerecht, Kap. 9 Rn. 35a; Herzog/Pruns, Der digitale Nachlass in der Vorsorge- und Erbrechtspraxis, S. 87.

323 Ebd. Fn. 322.

324 Zweifelnd auch Leipold in: MüKo BGB, $\$ 1922$ Rn. 34 und Martini/Kienle, JZ 2019, 235, 235; schon Martini, JZ 2012, 1145, 1150 spricht von einem im Vergleich zum Tagebuch „ungleich detailreichere[n] Wissensschatz eines OnlineKontos."; nur im Ansatz ebenso Bock in: Groll/Steiner (Hrsg.), Praxishandbuch Erbrechtsberatung, Rn. 20.28 und dies., AcP (217) 2017, 370, 394: „neue Quantität an Datenmassen und vermeintlich persönlichen Inhalten im digitalen Zeitalter." 
lassers erhält, liest nicht nur - um im Bilde zu bleiben - einzelne aufbewahrte Briefe, die der Verstorbene von Dritten erhalten hat. Zumindest im Falle von Social Media-affinen Nutzern ermöglicht der Zugang darüber hinaus tiefe Einblicke in eine umfassende, lückenlose Dokumentation nahezu der gesamten Lebensrealität des Verstorbenen und damit auch derjenigen seiner Kontakte. ${ }^{325}$ Die Kommunikationsdaten sind eher mit einem detaillierten Logbuch zu vergleichen, in dem jede Interaktion zwischen dem Erblasser und seinen Kommunikationspartnern notiert ist.

$\mathrm{Zu}$ den Informationen gehören der genaue Zeitpunkt des Abschickens sowie der Kenntnisnahme durch den Empfänger jeder einzelnen Nachricht, der gesamte Kommunikationsverlauf vergleichbar einem transkribierten Telefonat (und nicht - wie bei Briefpost - nur maximal die Hälfte der ausgetauschten Nachrichten) und bei manchen Internetdiensten auch Informationen zum physischen Ort, von dem die Nachrichten verschickt wurden. Die Kommunikationsdaten enthalten im Vergleich zur Briefpost ein Vielfaches an Inhalten samt zahllosen Fotografien, Sprachnachrichten, Links und Dateien. Weitere technische Entwicklungen wie die vollständige Aufzeichnung und Archivierung eines jeden Videotelefonats sind zukünftig leicht vorstellbar. ${ }^{326}$ Die sehr intensive Betroffenheit des Persönlichkeitsrechts der Kommunikationspartner kann sich in qualitativer Hinsicht ergeben, wenn sich unter den vielen Nachrichten intime Inhalte oder Bilder befinden. ${ }^{327}$ Aber auch in quantitativer Hinsicht ist der schieren Menge an Informationen und der Tatsache, dass sich daraus ein (vollständiges) Persönlichkeitsprofil des Nutzers ableiten ließe, persönlichkeitsrechtliche Bedeutung beizumessen. ${ }^{328}$

325 Nachdrücklich in diesem Sinne das Gutachten der Datenethikkommission vom 23.10.2019, S. 110, abrufbar a.a.O. (Fn. 30).

326 Auch Wagner sieht ein Risiko, dass jede auch nur „vorübergehende Parteiinteraktion für alle Ewigkeit gespeichert und den Erben zugänglich gemacht" werde, zitiert nach dem Diskussionsbericht von Bornhauser, AcP (218) 2018, 594, 595.

327 Den Kernbereich der privaten Lebensgestaltung, also die Intimsphäre, sieht Pruns, ErbR 2018, 550, 555 f. und ders., ErbR 2018, 614, 619 berührt bei Nacktbildern, statt Unvererbbarkeit folge daraus aber nur ein Löschungsanspruch gegen die Erben; zustimmend Leipold in: MüKo BGB, $\mathbb{S} 1922$ Rn. 38. Für ein anderes Beispiel intimer Inhalte s. bereits den Nachweis in Fn. 293.

$328 \mathrm{Zu}$ Detailreichtum als Faktor bei der Abgrenzung von Privat- und Intimsphäre vgl. Wanckel in: Götting/Schertz/Seitz (Hrsg.), Handbuch Persönlichkeitsrecht, $\$ 19$ Rn. 7 und 51 f. jew. m.w.N. 
bb) Erwartungen des verständigen Nutzers

Weiterhin argumentiert der BGH mit einer spezifischen Erwartungshaltung, die er verständigen Facebook Nutzern unterstellt. Zunächst könne der Kommunikationspartner schon zu Lebzeiten des Erblassers keine berechtigte Erwartung an die Vertraulichkeit der gemeinsamen Korrespondenz haben. ${ }^{329}$ Denn der Erblasser und Empfänger der Nachricht könne seine Login-Daten an Dritte weitergeben, Nachrichten weiterleiten oder auf andere Weise Dritten zugänglich machen. Ferner könnten Unbefugte ein Empfängerkonto von vornherein unter falschem Namen führen. ${ }^{330}$ Wenn der Kommunikationspartner aber bereits vor dem Erbfall das Risiko trage, dass Dritte Kenntnis von den im Account des Erblassers gespeicherten Informationen erwerben, gelte dies erst recht für den Zugriff der Erben auf diese Inhalte. ${ }^{331}$

Dem ist sicher zuzugeben, dass jeder Nutzer ein gewisses Risiko für seine Privatsphäre bewusst in Kauf nimmt, wenn er Soziale Medien verwendet. Gleichwohl sind sowohl der Ausgangspunkt des BGH als auch die daraus gezogene Konsequenz kritisch zu würdigen. Das Gericht geht von einem sehr ausgeprägten Risikobewusstsein des verständigen Nutzers aus. Inwiefern das der Lebensrealität von (vor allem jungen) ${ }^{332}$ Nutzern entspricht, sei dahingestellt. Sicher falsch wäre es aber zu glauben, dass ein Nutzer angesichts des ihm bewussten Risikos nur solche Inhalte über Soziale Medien mit dem Erblasser teilt, die er (notfalls) auch gegenüber den Erben oder sonstigen Dritten preisgeben würde. Vielmehr vertraut der verständige Nutzer trotz des ihm bewussten Risikos darauf, dass die Vertraulichkeit der Korrespondenz regelmäßig gewahrt bleibt.

Angesichts dessen lassen sich zwei Punkte festhalten: Zum einen sind die geteilten Inhalte trotz des etwaigen Risikobewusstseins persönlichkeits-

329 BGH NJW 2018, 3178 Rn. 41; zustimmend Preuß, NJW 2018, 3146, 3147; nach Gomille, ZUM 2018, 660, 662 besteht gegen die „Redseligkeit der selbst gewählten Vertrauensperson" weder zu deren Lebzeiten noch nach ihrem Tod ein Schutz.

330 Ebd. Fn. 329. Zu ergänzen ist noch die Gefahr, dass der Account des Erblassers trotz angemessener Sicherheitsvorkehrungen von Dritten gehackt wird.

331 BGH NJW 2018, 3178 Rn. 42; die Kenntnisnahme der Kommunikationsinhalte durch die Erben sei eine „im Internet typische Nutzungshandlung“, befindet Specht-Riemenschneider in: Röhricht/Graf von Westphalen/Haas (Hrsg.), HGB, Plattformnutzungsverträge, Rn. 95

332 Alexander, notar 2018, 367, 367 sieht bei ,jüngeren und besonders internetaffinen Generationen" häufig eine Verdrängung der Fragen des digitalen Nachlasses. 
rechtlich sensibel. Zum anderen verkennt der BGH die Ausnahme zur Regel, wenn aus den Risiken (die eintreten können, aber regelmäßig nicht eintreten) auf die Übergangsfähigkeit im Erbfall (der zwangsläufig eintritt) geschlossen wird. ${ }^{333}$ Die Vererbbarkeit des Accounts damit zu begründen, dass der Kommunikationspartner mit dem Eintritt der Erben in den Nutzungsvertrag des Erblassers (ergo mit der Vererbbarkeit) rechnen muss, ${ }^{334}$ mutet zirkelschlüssig an. ${ }^{335}$

cc) Keine Verfügungsbefugnis nach Versenden einer Nachricht

Schließlich, so der BGH, begebe sich der Nutzer mit dem Versenden jeder Verfügungsbefugnis über seine Nachricht und tue dies im Bewusstsein, dass er von da an grundsätzlich keine Möglichkeit habe, die übermittelte Nachricht zurückzufordern. ${ }^{336}$

Die Entscheidung des BGH ist in Bezug auf die gegenwärtige ${ }^{337}$, technische Ausgestaltung von Facebook sowie auf das entsprechend ausgerichtete Nutzungsverhalten ergangen. Insoweit ist der Entscheidung zuzustimmen. Allerdings sollte das Argument nicht pauschal auf andere Internetdienste übertragen werden, ${ }^{338}$ ohne dass die konkrete technische Ausgestaltung und die damit einhergehende Erwartungshaltung der Nutzer jeweils neu geprüft wird. So ermöglicht beispielsweise WhatsApp dem Absender innerhalb der ersten Stunde nach dem Verschicken, seine Nachricht auch mit Wirkung für die App des Empfängers nachträglich zu löschen. Der Inhalt wird selbst dann ausgeblendet, wenn der Empfänger die Nachricht zu diesem Zeitpunkt bereits gelesen hatte. ${ }^{339}$ Eine Erweiterung dieser Funktion über den Zeitraum von einer Stunde hinaus dürfte dem

333 Martini/Kienle, JZ 2019, 235, 238.

334 So ausdrücklich BGH NJW 2018, 3178 Rn. 44.

335 Leipold in: MüKo BGB, $\mathbb{S} 1922$ Rn. 34.

336 BGH NJW 2018, 3178 Rn. 41; überwiegend zustimmend Pruns, ErbR 2018, 614, 618; zweifelnd Martini/Kienle, JZ 2019, 235, 238.

337 Das Urteil ist vom 12. Juli 2018. Seitdem hat sich an der hier interessierenden Funktionsweise von Facebook (noch) nichts geändert [Stand: 17.11.2020].

338 Für das Urteil jedenfalls im Ganzen so aber Leipold in: MüKo BGB, $\$ 1922$ Rn. 46; Apel, ZD 2018, 486, 487 f.; Gloser, DNotZ 2018, 859, 865; Lieder in: Erman, $\mathbb{1} 1922$ BGB Rn.14a; ders./Berneith, FamRZ 2018, 1486, 1486 f.; Ludyga, ZEV 2018, 592, 592 f.; hingegen wie hier den Einzelfall betonend Hoeren, MMR 2018, 749, $749 \mathrm{f}$.

339 Weitere Informationen unter https://faq.whatsapp.com/de/iphone/26000069 [17.11.2020]. Davon freilich nicht erfasst sind Inhalte, die der Empfänger zuvor 
Anbieter in technischer Hinsicht leicht möglich sein. Auch der Erfolg von Snapchat beruht unter anderem darauf, dass Nutzer einander Nachrichten schicken können, die der Empfänger nur vorübergehend aufrufen kann. ${ }^{340}$ Zum einen folgt daraus, dass es durchaus technisch möglich ist, dem Nutzer auch nach dem Versenden einer Nachricht weiter eine (begrenzte) Verfügungsbefugnis über dieselbe einzuräumen. Zum anderen wird die Erwartungshaltung der Nutzer durch Internetdienste, die entsprechende Einflussmöglichkeiten technisch einräumen, (mit)geprägt. Dabei ist zwischen den verschiedenen Apps sowie innerhalb einer App nach unterschiedlichen Versionen zu unterscheiden. ${ }^{341}$ Insgesamt macht die eröffnete Bandbreite der Funktionen es schwer, dienstübergreifend mit einer bestimmten Erwartungshaltung der Nutzer an die eigene Verfügungsbefugnis zu argumentieren.

\section{dd) Zwischenergebnis zu c)}

Nach der Entscheidung des BGH haben die Interessen der Kommunikationspartner des Erblassers zurückzustehen hinter den Interessen der Erben und dem Kontinuitätsgedanken des $₫ 1922$ Abs. 1 BGB. Das Ergebnis des Gerichts, wonach die Persönlichkeitsrechte der Kommunikationspartner der Vererbbarkeit des Nutzungsvertrags nicht entgegenstehen, ist sehr ausführlich begründet und im Wesentlichen nachvollziehbar. Dennoch zeigen nach hier vertretener Ansicht die Wertungen des BGH dort einige Schwächen, wo sie die Realität von Social Media-affinen Nutzern nicht in vollem Umfang nachvollziehen. Internetdienste sind nicht (nur) das digitale Äquivalent zum klassischen Briefverkehr. Dieser Vergleich sollte nicht den Blick auf Ausmaß, Vielfalt und Dynamik des realen Nutzungsverhaltens verstellen. Selbst wenn man aber die dargestellten Einschätzungen des BGH in Bezug auf den Nutzungsvertrag zu Facebook teilt, darf das Ergebnis gleichwohl nicht vorschnell auf andere oder gar auf grundsätzlich alle accountgestützten Nutzungsverträge übertragen werden. Vielmehr sollten

bereits außerhalb der App (zusätzlich) abgespeichert hat. Das dürfte aber die Ausnahme sein.

340 Klages, ZD 2017, 390, 390 zufolge zeige das heutige Nutzerverhalten, dass Inhalte, die keinen Bestand haben sollen, mit Diensten wie Snapchat verschickt würden, da dort Inhalte selbstständig unverzüglich nach Kenntnisnahme gelöscht werden.

341 WhatsApp etwa lässt das nachträgliche Löschen nur für Nutzer zu, welche die aktuellste Version der Anwendung installiert haben (s. dazu a.a.O. Fn. 339). 
die vonseiten des BGH unterstellten Erwartungen der Nutzer eines Internetdienstes für jedes Angebot einzeln geprüft werden.

Im Übrigen ist es eine Schwäche von $\$ 399$ BGB als Prüfungsmaßstab, dass er als Ergebnis nur die Extrempositionen von Vererbbarkeit und Unvererbbarkeit und in der Folge vollen oder gar keinen Zugang zu den Accountinhalten zulässt. Wie sich zeigen wird, erlaubt namentlich das Datenschutzrecht bei der Entscheidung über den Zugang auch Zwischenpositionen. Deshalb bietet das Datenschutzrecht sich vorrangig an, um die widerstreitenden Interessen von Erben, Provider und Kommunikationspartnern zu einem schonenden Ausgleich zu bringen. ${ }^{342}$

\section{d) Reichweite des ererbten Rechts am Nutzungskonto}

Interessanterweise lässt der BGH in seiner Entscheidung, in der er ansonsten gleich mehrfach durch obiter dicta Stellung bezieht, ${ }^{343}$ offen, ob das Erbrecht über den (schlichten) Zugang zu den Accountinhalten hinaus auch das Recht zur fortgesetzten Nutzung des Accounts durch die Erben einschließt. ${ }^{344}$ Wie bereits aufgezeigt, nimmt der BGH in Bezug auf die Übermittlung von Nachrichten und den Kontozugang eine formal-technische Betrachtungsweise vor und folgert daraus nur eine Konto-, aber keine Personenbezogenheit der Providerpflichten. ${ }^{345}$ Die aktive Weiternutzung des Kontos - also das Posten und Verschicken von Nachrichten - soll hingegen ,auf den Kontoberechtigten zugeschnitten und damit personenbezogen" sein. ${ }^{346}$ Diese Widersprüchlichkeit scheint das Gericht in Kauf zu nehmen, weil es ohnehin von einer geringen praktischen Relevanz der aufgeworfenen Frage ausgeht: Die Fortführung des Kontos durch die Erben sei „regelmäßig nicht beabsichtigt. “347 Diese Annahme wird aber nicht

342 Hoeren, MMR 2018, 749, 749; Martini/Kienle, JZ 2019, 235, 238 f. betonen zurecht, dass die DS-GVO etwa mit der Anonymisierung oder Pseudonymisierung von Nutzerdaten vermittelnde Zwischenpositionen kennt. Vgl. dazu S. $133 \mathrm{f}$.

343 BGH NJW 2018, 3178 Rn. 28 ff., 47 ff., 52 f. Die obiter dicta loben wegen ihrer klarstellenden Wirkung Apel, ZD 2018, 486, 486 und Gloser, DNotZ 2018, 859, 865.

344 BGH NJW 2018, 3178 Rn. 36.

345 BGH NJW 2018, 3178 Rn. 35; vgl. dazu bereits auf S. 95 f.

346 Ebd. Fn. 344; dieser Tendenz folgend gegen ein Recht auf aktive (Weiter-)Nutzung durch die Erben Leipold in: MüKo BGB, $\$ 1922$ Rn. 37.

347 Zwei Mal explizit so BGH NJW 2018, 3178 Rn. 36 und 37; diese Einschätzung teilt auch Seidler, Digitaler Nachlass, S. 136; dies, NZFam 2020, 141, 144 f. 
weiter belegt oder begründet. Dabei ließen sich mit dem YouTube-Channel oder dem Instagram Account eines Influencers oder mit einem gut bewerteten Google Business Account schon heute mehrere Beispiele nennen, bei denen die zukünftige Nutzung des Kontos einen erheblichen Wert haben kann und sogar noch vor dem Zugang zu gespeicherten Inhalten aus der Vergangenheit das Hauptinteresse der Erben bilden könnte. ${ }^{348}$ Die Entwicklung der technischen Möglichkeiten und der Nutzungsgewohnheiten dürfte weitere solche Anwendungsfälle mit sich bringen. Der Standpunkt des BGH wird indessen nachvollziehbarer, wenn man mit dem Vergleich zum Giro-Konto ein Argument aus der vorausgegangenen Debatte genauer in den Blick nimmt. Denn der BGH zitiert hier selbst seine Rechtsprechung zum Girovertrag sowie die darauf Bezug nehmenden Beiträge zum digitalen Nachlass. ${ }^{349}$

aa) Die Rechtsprechung zum Girokonto im digitalen Nachlass

Den Vergleich zur Rechtsprechung des BGH zum Erbe an Girokonten ${ }^{350}$ brachten ursprünglich Bräutigam ${ }^{351}$ und Herzog ${ }^{352}$ - allerdings mit gegensätzlichen Ergebnissen - in die Debatte zum digitalen Nachlass ein. Als Mitautoren der Stellungnahme des Deutschen Anwaltvereins ${ }^{353}$ haben sie auf der Suche nach einer praktikablen Handhabung des digitalen Nachlasses die (vermeintlich) vergleichbare Unterscheidung zwischen Kontoinhaberschaft und Kontoinhalt (= Guthaben) herangezogen. Bräutigam zufolge sei die Inhaberschaft eines Girokontos wegen der ungehinderten Eröffnung für jedermann gerade nicht vermögensrechtlicher (und im Umkehrschluss daher höchstpersönlicher) Natur. ${ }^{354}$ Daraus schlussfolgert er ers-

348 Dass rein geschäftlich genutzte Accounts ggf. anders zu behandeln sind, sieht schon Raude, RNotZ 2017, 17, 20. Freilich können sich sodann Abgrenzungsschwierigkeiten in der Praxis ergeben. Siehe dazu bereits den Hinweis auf die Schlussanträge von Generalanwalt Bobek vom 14.11.2017 - C-498/16, Rdnr. 49 Maximilian Schrems/Facebook in Fn. 235.

349 Ebd. Fn. 344.

350 BGH NJW 1996, 190; BGH NJW 2000, 1258; Weidlich in: Palandt, $\mathbb{} 1922$ BGB Rn. 30; instruktiv zur hiesigen Debatte s. Seidler, Digitaler Nachlass, S. $74 \mathrm{ff}$.

351 Bräutigam in: DAV-Stellungnahme Nr.34/2013, S. 17 ff. und ders. in: Burandt/ Rojahn (Hrsg.), nach $\$ 1922$ BGB Rn. 5.

352 Herzog in: DAV-Stellungnahme Nr.34/2013, S.36f. und 51 und dies., NJW 2013, 3745, 3747, 3749.

353 DAV-Stellungnahme Nr. 34/2013.

354 Ebd. Fn. 351. 
tens eine Übertragbarkeit auf digitale Nutzungskonten, da deren Eröffnung ebenfalls jedermann problemlos möglich ist, und zweitens ein Hindernis für die Rechtsnachfolge in die Inhaberschaft selbst. ${ }^{355}$ Herzog hingegen betont zurecht, dass nach der Rechtsprechung des BGH sowohl die Inhaberschaft als auch das Guthaben des Girokontos zumindest im Zeitpunkt des Erbfalls auf die Erben übergehen und mithin vererbbar sind. ${ }^{356}$ Daraus folgert sie, dass die Giro-Rechtsprechung keinen Schluss auf die Unvererbbarkeit digitaler Accounts zulässt. ${ }^{357}$

In seiner aktuellen Entscheidung zur Übergangsfähigkeit des FacebookNutzungsverhältnisses will der BGH augenscheinlich vor allem das von Bräutigam vorgetragene Argument gegen die Vererbbarkeit entkräften, indem er sich Herzogs Lesart der Giro-Rechtsprechung anschließt. ${ }^{358}$ Insofern reiht sich dieser Teil nahtlos in die sukzessions- und erbenfreundliche Argumentationslinie des gesamten Facebook-Urteils ein. Indem aber auf die alte Rechtsprechung zum Giroverhältnis rekurriert wird, um damit dem Argument von Bräutigam entgegenzutreten, wird unglücklicherweise auch ein anderer Aspekt der Giro-Judikate in die Debatte zum digitalen Nachlass hineingetragen. Denn die Girorechtsprechung sieht vor, dass bei fortgesetzter Nutzung des ererbten Kontos anstelle des alten Girovertrags (zwischen Erblasser und Bank) ein neues, eigenes Rechtsverhältnis zwischen dem Erben und der Bank entsteht. Dies ist dem konkreten Sachverhalt, welcher der damaligen Entscheidung zugrunde lag, geschuldet. ${ }^{359}$ Die Übertragung dieses Gesichtspunkts auf die Situation des digitalen Nachlas-

355 Hierunter versteht Bräutigam die fortgesetzte Nutzung des Accounts durch die Erben. Gleichzeitig geht er von einem problemlosen Übergang der Accountinhalte auf die Erben aus. Vgl. hierzu die Fundstellen in Fn. 351. Dem folgt Leeb, K\&R 2014, 693, $694 \mathrm{f}$.

356 Ebd. Fn. 352. Implizit wirft sie damit Bräutigam vor, die Giro-Rechtsprechung falsch zu verstehen.

357 Dem schließt sich die ganz h.M. an, s. Gloser, MittBayNot 2016, 12, 14; Budzikiewicz, AcP (218) 2018, 558, 569 f.; Kutscher, Der digitale Nachlass, S. 101; Seidler, Digitaler Nachlass, S. 87; Herzog/Pruns, Der digitale Nachlass in der Vorsorgeund Erbrechtspraxis, S. 75; BGH NJW 2018, 3178 Rn. 36.

358 BGH NJW 2018, 3178 Rn. 36.

359 Herzog, NJW 2013, 3745, 3747 bezeichnet die Entscheidung als „Sonderfall“; deutlicher noch Batreau in: NK-BGB, Anhang II zu $\mathbb{1 9 2 2}$ BGB Rn.3, der von einer „atypischen Sonderkonstellation“ und einem "Sonderweg“ des BGH spricht, den die Bankenpraxis als „Ausflug der höchstrichterlichen Rechtsprechung weitgehend unbeachtet gelassen" habe. Inhaltlich ging es in dem Urteil um eine Vor- und Nacherbschaft, bei der die Vorerbin das Konto des Erblassers siebzehn Jahre zur Abwicklung der eigenen Geschäfte des täglichen Lebens verwendete. Der Nacherbe versuchte sodann erfolglos, die kontoführende Bank 
ses ist der Ursprung dafür, dass der BGH sich nun mit seinen Zweifeln an dem Recht der Erben zur weiteren Nutzung des Erblasseraccounts sowohl zu seiner sonstigen sehr erbenfreundlichen Linie, als auch zu seiner formal-technischen Betrachtungsweise des Nutzungsverhältnisses in Widerspruch setzt. ${ }^{360}$ Es ist daher zu prüfen, ob die Parallele zum Girokonto nicht insgesamt aufgegeben werden kann.

bb) Keine Vergleichbarkeit von Girokonto und online Accounts

Girokonten samt Guthaben einerseits und digitale Nutzerkonten mit ihren gespeicherten Inhalten andererseits unterscheiden sich hinsichtlich ihrer Funktion, ihrer rechtlichen Qualität und ihres (potenziellen) Wertes.

Die Funktion eines Girokontos liegt (fast) ausschließlich in der Abwicklung des Zahlungsverkehrs des Kontoinhabers. Der Kontoinhaberschaft liegt ein Girovertrag zugrunde, der ein Zahlungsdiensterahmenvertrag im Sinne von $\$ 675 \mathrm{f}$ Abs. 2 BGB ist. ${ }^{361}$ Der Inhaberschaft selbst kommt aber kein Wert zu, da gem. $\$ 31$ Abs. 1 ZKG jeder Verbraucher mit rechtmäßigem Aufenthalt in der Europäischen Union einen Anspruch auf Abschluss eines Basiskontovertrags hat. ${ }^{362}$ Es sind kaum Konstellationen vorstellbar, in denen eine bestimmte Nutzung des Kontos durch seinen Inhaber zu einem gesteigerten Wert der Kontoinhaberschaft selbst führen kann. An dem Guthaben wiederum besteht unstreitig ein Auszahlungsanspruch des Kontoinhabers gegen die Bank gem. $\$ \$ 780,781$ BGB. ${ }^{363}$

Die den Accounts bei Sozialen Medien und anderen Internetdiensten zugrundeliegenden Nutzungsverträge sind juristisch weitaus weniger klar

u.a. auf Umschreibung des Kontos auf sich selbst in Anspruch zu nehmen, vgl. BGH NJW 1996, 190; siehe zum Ganzen bereits die Darstellung bei Seidler, Digitaler Nachlass, S. $74 \mathrm{ff}$.

360 So verhält es sich auch mit Herzog/Pruns, Der digitale Nachlass in der Vorsorgeund Erbrechtspraxis, S. 75 f., die in (fast) allen Fragen sukzessionsfreundlich argumentieren und nur bei der Weiterführung des Accounts insoweit zweifeln; Herzog in: NK-Nachfolgerecht, Kap. 9 Rn. 27; s. ferner Raude, RNotZ 2017, 17, 20.

361 Vgl. nur Herresthal in: MüKo HGB, Teil 1. Recht des Zahlungsverkehrs, A. Das Giroverhältnis, Rn. 167 m.w.N. sowie passim zum Giroverhältnis allgemein.

362 Zum Kontrahierungszwang der Kreditinstitute vgl. Herresthal, a.a.O. (Fn. 361), Rn. 185 ff.; mit der Abschlusspflicht argumentiert auch Fußeder, Soziale Netzwerke im Nachlass, S. $59 \mathrm{f}$.

363 Herresthal, a.a.O. (Fn. 361), Rn. 350 m.w.N. zur a.A. 
definiert. ${ }^{364}$ Die Funktion der Accounts ist nach der Intention beider Vertragsparteien vielfältig und entwicklungsoffen. ${ }^{365}$ Funktional schließt der Account private und gewerbliche Zwecke ein, umfasst private und öffentliche Kommunikation sowie politische und sonstige Meinungsbildung. Die Rechtsposition an den abgespeicherten Inhalten ist, wie gesehen, ${ }^{366}$ in Ermangelung eines Rechts an Daten unklar und setzt sich aus vereinzelten Immaterialgüterrechten und lediglich faktischen Zugangsmöglichkeiten zusammen. Der Wert der Kontoinhaberschaft ist im Ausgangspunkt und Regelfall sehr gering, kann aber durch eine bestimmte Nutzung des Accounts stark zunehmen. ${ }^{367}$

Nach alledem handelt es sich bei Girokonto und digitalem Account um zwei grundlegend verschiedene Dinge. Zwar mag der Vergleich gerade ganz zu Beginn der Debatte um den digitalen Nachlass einen ersten kreativen Zugang zu der Problematik eröffnet haben, weil hier wie dort zwischen der Inhaberschaft selbst und dem Kontoinhalt differenziert werden kann. ${ }^{368}$ Insgesamt sollte aber über die Reichweite des Erbrechts an digitalen Nutzungskonten autonom entschieden werden, anstatt alte und Sonderkonstellationen betreffende Rechtsprechung zum Girokonto heranzuziehen.

\section{cc) Zwischenergebnis zu d)}

Sofern das Nutzungsverhältnis grundsätzlich vererbbar ist, schließt das Erbrecht daran neben dem Zugang zu gespeicherten Inhalten auch die aktive Weiternutzung des Accounts ein. Es wäre widersprüchlich, die Vererbbarkeit im Grundsatz zu bejahen, woraus der persönlichkeitsrechtlich sensible Zugang zu allen gespeicherten Inhalten folgt, sodann aber mit Hin-

$364 \mathrm{Zu}$ den Schwierigkeiten bei der vertragstypologischen Einordnung vgl. bereits zuvor auf S. $33 \mathrm{ff}$. Wie gesehen, ist allein schon umstritten, ob es sich überhaupt um ein entgeltliches Vertragsverhältnis handelt.

365 Enger noch und nur für E-Mail-Accounts Seidler, Digitaler Nachlass, S. 87 f.

366 Zum Recht an Daten s. S. 44 ff.

367 Fußeder, Soziale Netzwerke im Nachlass, S. 58 f.; Seidler, NZFam 2020, 141, 144; zur Funktionsweise des Influencings vgl. bereits zuvor auf S. $74 \mathrm{ff}$. Ein weiteres Beispiel dafür sind Accounts und virtuelle Gegenstände bei online Computerspielen, vgl. Hermes, GRUR-Prax 2013, 400, $400 \mathrm{ff}$.

368 Seinerzeit wurde aber auch, ebenfalls von Herzog, die (Un-)Vererbbarkeit eines Mietverhältnisses hilfsweise als Argument herangezogen, vgl. Herzog in: DAVStellungnahme Nr. 34/2013, S. 37 ff. Beide Vergleiche dürften als erste Orientierungshilfen in der noch sehr jungen Debatte zu sehen sein. 
weis auf den Personenbezug die fortgesetzte Nutzung des Accounts als vom Erbrecht nicht umfasst anzusehen. ${ }^{369}$

Unabhängig von diesem Ergebnis kann der Umgang des Erben mit seinem Recht durchaus bestimmten Beschränkungen unterliegen, die den Übergang selbst nicht betreffen. So ist bei der geschäftlichen Nutzung eines geerbten Accounts das Lauterkeitsrecht zu beachten. Denn es verstößt etwa gegen $₫ 5$ Abs. 1 S. 2 Nr.3 UWG, wer in irreführender Weise bestimmte Tätigkeits- oder Berufsbezeichnungen verwendet oder unrichtige Angaben über besondere Fähigkeiten oder Leistungen macht. ${ }^{370}$ Der Erbe muss hier also überprüfen, ob alle Angaben in dem Account des Erblassers auch auf ihn zutreffen oder ob gegebenenfalls deutlich wird, dass keine Personenidentität zwischen altem und neuem Accountinhaber besteht. Verarbeitet der Erbe in einem geschäftlichen Account, der auf ihn übergegangen ist, beispielsweise Kundendaten, können ihn auch datenschutzrechtliche Pflichten bei der Accountnutzung treffen. ${ }^{371}$ Unabhängig von der Frage, ob der Account geschäftlich oder privat genutzt wird, können schließlich Klarnamenpflichten des (neuen) Accountinhabers bestehen. ${ }^{372}$

e) Zwischenergebnis zu 1.

Im Verhältnis zwischen dem Erblasser, seinen Kommunikationspartnern und dem Provider ist nach der Rechtsprechung des BGH für den Fall von

369 Anders wohl BGH NJW 2018, 3178 Rn. 21 und 36; Herzog/Pruns, Der digitale Nachlass in der Vorsorge- und Erbrechtspraxis, S. 75 f.; Leipold in: MüKo BGB, $\$ 1922$ Rn. 33 und 37; Biermann in: Münchener Anwaltshandbuch Erbrecht, \50 Rn. 14; ders., ErbR 2018, 577, 578; ebenso aber mit anderer Begründung Budzikiewicz, AcP (218) 2018, 558, 571; Rössel, ITRB 2018, 224, 225 weist auf die Identifikationsfunktion des Nutzungskontos hin, die beeinträchtigt würde.

370 Bornkamm/Feddersen in: Köhler/Bornkamm/Feddersen (Hrsg.), \$5 UWG

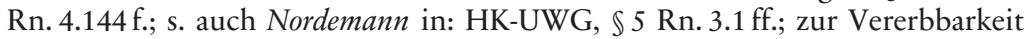
von Bewertungen vgl. Herzog in: NK-Nachfolgerecht, Kap. 9 Rn. 36 sowie Bericht der Arbeitsgruppe „Digitaler Neustart“ der Konferenz der Justizministerinnen und Justizminister der Länder, S. 360 ff., abrufbar a.a.O. (Fn. 43).

$371 \mathrm{Zu}$ dieser Konstellation vgl. die Studie des Fraunhofer Instituts für Sichere Informationstechnologie, S. 46, abrufbar a.a.O. (Fn. 7).

372 Seidler, NZFam 2020, 141, 144; Herzog/Pruns, Der digitale Nachlass in der Vorsorge- und Erbrechtspraxis, S. 76. Diese Pflicht kann sich z.B. aus den AGB des Providers ergeben, vgl. BGH NJW 2018, 3178 Rn. 37 zu Facebook (nach dessen Angaben); ferner Lieder/Berneith, FamRZ 2016, 743, 743 und Studie des Fraunhofer Instituts für Sichere Informationstechnologie, S.47, abrufbar a.a.O. (Fn. 7). 
Facebook keine die Vererbbarkeit des Nutzungsvertrags ausschließende Höchstpersönlichkeit der Leistungspflichten anzunehmen. Als Maßstab hat das Gericht hier den Rechtsgedanken des $\$ 399$ Fall 1 BGB herangezogen. Dem ist im Grundsatz zuzustimmen. Bevor dieser Grundsatz aber auf andere Internetdienste übertragen wird, sind die Annahmen, die den BGH für Facebook zu diesem Ergebnis kommen ließen, jeweils auf ihre Gültigkeit zu überprüfen. Das betrifft insbesondere den Persönlichkeitsrechtsschutz der Kommunikationspartner des Erblassers und die Erwartungen eines verständigen Nutzers, die sich zwischen verschiedenen Internetdiensten unterscheiden (können). Hier müssen die dynamische Entwicklung der technischen Möglichkeiten sowie das darauf basierende Nutzungsverhalten fortlaufend beobachtet werden. Den Regelfall wird dabei die Vererbbarkeit des Nutzungsverhältnisses bilden, dem nur ausnahmsweise die Unvererbbarkeit wegen angenommener Höchstpersönlichkeit gegenübersteht. Positionen, die zwischen diesen beiden Polen liegen, sind nicht auf der Ebene des Rechtsübergangs, sondern vielmehr im Rahmen des Umgangs mit der bereits übergegangen Position - etwa unter dem Gesichtspunkt des Datenschutzrechts - an späterer Stelle zu erarbeiten.

Wenn und soweit ein Nutzungsverhältnis unter dem Gesichtspunkt des $\$ 399$ Fall 1 BGB als vererbbar beurteilt wird, schließt das Erbrecht auch die aktive Weiternutzung des übergegangenen Accounts ein. Dies kann nicht unter neuerlicher Bezugnahme auf das Merkmal der Höchstpersönlichkeit in Zweifel gezogen werden. Wiederum getrennt zu betrachten ist die Frage, ob und inwieweit der Erbe bei der Nutzung des ererbten Accounts Beschränkungen des geltenden Rechts zu beachten hat. Die Rechtsprechung des BGH zur Vererbbarkeit eines Girokontos kann auf die hier aufgeworfenen Fragen nicht angewendet werden und sollte in der Debatte um den digitalen Nachlass als Argumentationshilfe insgesamt aufgegeben werden.

2. Höchstpersönlichkeit im Verhältnis zwischen dem Erblasser, seinen nächsten Angehörigen und den Erben

Im Verhältnis zwischen dem Erblasser und seinen Erben bzw. seinen nächsten Angehörigen kann das Merkmal der Höchstpersönlichkeit ebenfalls Bedeutung erlangen. Dass zwischen den Erben und den nächsten An- 
gehörigen gegebenenfalls zu differenzieren ist, ${ }^{373}$ zeigt der folgende Blick auf die Grundsätze des allgemeinen respektive des postmortalen Persönlichkeitsrechts.

Das allgemeine Persönlichkeitsrecht jeder natürlichen Person ergibt sich zu ihren Lebzeiten aus Art. 2 Abs. 1 in Verbindung mit Art. 1 Abs. 1 GG. ${ }^{374}$ Nach ihrem Tod besteht das sich nunmehr aus Art. 1 Abs. 1 GG allein ergebende postmortale Persönlichkeitsrecht fort. ${ }^{375}$ Dessen sachlicher Schutzbereich erfasst zum einen den allgemeinen Achtungsanspruch, der dem Menschen kraft seines Personseins zusteht, und zum anderen den sittlichen, personalen und sozialen Geltungswert, den eine Person durch ihre Lebensleistung erworben hat. ${ }^{376}$ Dieser Schutz bewahrt den Verstorbenen insbesondere davor, herabgewürdigt oder erniedrigt zu werden. ${ }^{377}$ Mit Blick auf die erbrechtliche Übergangsfähigkeit ist zwischen den vermögenswerten Bestandteilen des allgemeinen Persönlichkeitsrechts, die regulär vererblich sind, und dem nicht vererblichen ideellen Teil zu unterscheiden. ${ }^{378}$ Letzterer wird als postmortales Persönlichkeitsrecht von den Wahrnehmungsberechtigten subjektlos treuhänderisch für den und im (mutmaßlichen) Interesse des Verstorbenen ausgeübt. ${ }^{379}$ Die Wahrnehmungsberechtigten bestimmt der Erblasser selbst, in Ermangelung einer Anordnung gelten indessen die nächsten Angehörigen als ermächtigt. Zur näheren Bestimmung dieses Begriffs wird gemeinhin auf $\$ 77$ Abs. 2 StGB, $\$ 22$ S. 4 KUG und $\$ 60$ Abs. 2 UrhG verwiesen. ${ }^{380}$ Danach sind übereinstimmend zunächst der überlebende Ehegatte bzw. Lebenspartner und die Kin-

373 In der Facebook-Entscheidung des BGH kam es darauf nicht an, weil die Kläger zugleich Erben und nächste Angehörige waren, vgl. BGH NJW 2018, 3178 Rn. 48, 53, $92 \mathrm{f}$.

374 Lorenz in: Bonner Kommentar, Art. 2 Abs. 1 GG Rn. 228; Starck in: v. Mangoldt/ Klein/Starck, Art. 2 GG Rn. 86, 89; Sodan in: Sodan (Hrsg.), Art. 2 GG Rn. 9.

375 Lorenz in: Bonner Kommentar, Art. 2 Abs. 1 GG Rn.388; Sodan in: Sodan (Hrsg.), Art. 1 GG Rn. 26; Schmitt in: Götting/Schertz/Seitz (Hrsg), Handbuch Persönlichkeitsrecht, $\mathbb{} 29 \mathrm{Rn} .1 \mathrm{ff}$.

376 So fast wörtlich BVerfG NJW 2006, 3409, 3409; BVerfG NJW 2001, 2957, 2959.

377 BVerfG NJW 2001, 2957, 2959; BVerfG NJW 1971, 1645, 1647; ausführlich dazu Rixecker in: MüKo BGB, nach $\$ 12$ Rn. 46 ff., 54 ff.

378 Zum Ganzen Schmitt in: Götting/Schertz/Seitz (Hrsg.), Handbuch Persönlichkeitsrecht, $\mathbb{} 29$ Rn. 3 ff.; Leipold, Erbrecht, Rn. 635 f.; einschränkend Lange/Holtwiesche, ZErb 2016, 157, 160.

379 Ebd. Fn. 378; Herzog in: NK-Nachfolgerecht, Kap. 9 Rn. 50 m.w.N.

380 Lange, Erbrecht, Kap. 2 Rn. 40; Leipold in: MüKo BGB, $\$ 1922$ Rn. 158; ders., Erbrecht, Rn. 635; ausführlich Schmitt in: Götting/Schertz/Seitz (Hrsg.), Handbuch Persönlichkeitsrecht, $\$ 29$ Rn.57ff.; ständige Rechtsprechung seit BGH NJW 1968, 1773, 1775. 
der gemeinsam berechtigt. Subsidiär treten die Eltern des Erblassers an deren Stelle. Im Folgenden ist zu prüfen, ob aus alledem Rückschlüsse auf den Umgang mit dem digitalen Nachlass gezogen werden können.

\section{a) Differenzierungstheorie und „Infektion“}

Die soeben dargestellten und weithin anerkannten Grundsätze des postmortalen Persönlichkeitsschutzes eröffnen zum einen die Unterscheidung zwischen Erben und nächsten Angehörigen und zum anderen zwischen vermögenswerten und ideellen Bestandteilen der Persönlichkeit. Hieraus leitete zuerst Hoeren für den digitalen Nachlass eine Matrix ab, nach der vermögenswerte Inhalte des Erblasseraccounts den Erben und nicht-vermögenswerte, höchstpersönliche Inhalte den nächsten Angehörigen des Erblassers zuzuordnen seien. ${ }^{381}$ Rein private Nachrichten seien aus Gründen des postmortalen Persönlichkeitsschutzes den Erben vorzuenthalten und den nächsten Angehörigen als Vertrauenspersonen zur treuhänderischen Verwahrung zuzuleiten. ${ }^{382}$ Diese Unterscheidung werde auch durch den Wortlaut des $\$ 1922$ Abs. 1 BGB bestätigt, wonach (nur) das „Vermögen" des Erblasser auf die Erben übergeht. ${ }^{383}$ Auch Martini möchte den Accountinhalt in einen vermögensrechtlichen Teil und einen personenbezogenen Teil trennen. ${ }^{384}$ Während der erstere den Erben zustehe, stehe das postmortale Persönlichkeitsrecht für den personenbezogenen Teil jedweder Kenntnisnahme durch Erben wie durch Angehörige entgegen. Martini geht also insofern über Hoeren hinaus, wenn er schlussfolgert, dass der $\mathrm{Zu}$ gang zu dem nicht-vermögenswerten Teil des Accountinhalts mit dem Tod des Nutzers erlischt. ${ }^{385}$ Danach würde aus dem postmortalen Persönlich-

381 Hoeren, NJW 2005, 2113, $2114 \mathrm{f}$.

382 Hoeren, NJW 2005, 2113, 2114 nennt als Beispiel „Liebes- oder einfache Gruß-EMails“; dieser Ansicht folgen Brinkert/Stolze/Heidrich, ZD 2013, 153, $154 \mathrm{f}$. und Haase, Rechtsfragen des digitalen Nachlasses in: Taeger (Hrsg.), Tagungsband DSRI-Herbstakademie 2013, S. 385; Rixecker in: MüKo BGB, nach $₫ 12$ Rn. 151.

383 Ausdrücklich so Brinkert/Stolze/Heidrich, ZD 2013, 153, 154; Hoeren, NJW 2005, 2113, 2114 sieht darin „einen groben Auslegungsgrundsatz.“

384 Martini, JZ 2012, 1145, $1150 \mathrm{ff}$.

385 Martini, JZ 2012, 1145, 1150, das gelte vorbehaltlich anderslautender Verfügungen durch den Erblasser. 
keitsrecht ein Anspruch des Erblassers auf Schutz vor Einsicht seiner Erben und Angehörigen in seine persönlichen Nachrichten folgen. ${ }^{386}$

Ein Folgeproblem dieser Ansicht und der auch von ihren Vertretern eingestandenen Abgrenzungsprobleme wird unter dem Stichwort der Infektion diskutiert. ${ }^{387}$ Die naheliegende Konstellation, in der ein Account sowohl vermögenswerte als auch höchstpersönliche Nachrichten beinhaltet, provoziert die Frage, wer die erforderlich werdende Unterteilung treffen sollte und rechtlich dürfte. ${ }^{388}$ Wenn und soweit hier keine praktikable Lösung gefunden wird, könnte nach dieser Ansicht auch nur eine höchstpersönliche Nachricht den gesamten Account gleichsam infizieren und so zu dessen Unvererbbarkeit führen. Denn, so lässt sich die Argumentation konsequent fortsetzen, der Menschenwürdegehalt des postmortalen Persönlichkeitsrechts ist gem. Art. 1 Abs. 1 S. 1 GG unantastbar und daher keiner Abwägung z.B. in Form einer Schwerpunktbetrachtung zugänglich. ${ }^{389}$

\section{b) Einheitlichkeit des Nachlasses}

Der BGH lehnt die Differenzierungstheorie ab und sieht allein die Erben als Berechtigte an dem gesamten (digitalen) Nachlass an. ${ }^{390}$ Neben den erheblichen praktischen Problemen, die eine Trennung der Accountinhalte in vermögenswert und höchstpersönlich mit sich bringen würde (dazu aa), sprechen für eine solche Lesart die Wertung der $\$ \$ 2047$ Abs. 2, 2373 BGB (dazu bb) sowie die Funktion und Reichweite der Wahrnehmungsberech-

386 Martini, JZ 2012, 1145, 1152 sieht hier eine staatliche Schutzpflicht sowie einen Anspruch des Nutzers gegen den Diensteanbieter; Rixecker in: MüKo BGB, nach $\mathbb{} 12 \mathrm{Rn} .151$.

387 Bräutigam in: DAV-Stellungnahme Nr. 34/2013, S. 24; Rott/Rott, NWB-EV 2013, 160, 164; Raude, ZEV 2017, 433, 435; Bericht der Arbeitsgruppe „Digitaler Neustart" der Konferenz der Justizministerinnen und Justizminister der Länder, S. 343, abrufbar a.a.O. (Fn. 43); Kutscher, Der digitale Nachlass, S. 105 f.

388 Martini, JZ 2012, 1145, 1152 möchte die Provider in die Pflicht nehmen, die den zusätzlichen Aufwand dann einzupreisen hätten.

389 Zur Abwägungsfeindlichkeit der Menschenwürde vgl. Sodan in: Sodan (Hrsg.), Art. 1 GG Rn. 27; Dreier in: Dreier (Hrsg.), Art. 1 Abs. 1 GG Rn. 46; Jarass in: Jarass/Pieroth (Hrsg.), Art. 1 GG Rn. 15; zur Ableitung des postmortalen Persönlichkeitsrechts aus Art. 1 Abs. 1 GG vgl. bereits die Nachweise in Fn. 375.

390 BGH NJW 2018, 3178 Rn. 47 ff.; explizit in Bezug darauf zustimmend Lieder/ Berneith, FamRZ 2018, 1486, 1487; Wellenhofer, JuS 2018, 1101, 1103; Ludyga, ZEV 2018, 592, 593; Heintz/Ludyga, jM 2018, 398, 400. 
tigung nächster Angehöriger an dem postmortalen Persönlichkeitsrecht des Erblassers (dazu cc).

\section{aa) Praktikabilitätserwägungen}

Es bestehen in zweifacher Hinsicht Zweifel an der Praktikabilität der Differenzierungstheorie. ${ }^{391}$ Zum einen lässt sich die Unterscheidung zwischen vermögenswerten und höchstpersönlichen Accountinhalten inhaltlich nicht trennscharf durchführen. Vielmehr sind die Grenzen fließend, etwa weil höchstpersönliche Inhalte beispielsweise als Beweismittel in einem Prozess Vermögenswert erlangen können oder weil in einer Nachricht höchstpersönliche und geschäftliche Informationen gemeinsam enthalten sind. ${ }^{392}$ Zum anderen wäre, auch wenn geeignete inhaltliche Kriterien entwickelt würden, mit der Sortierung ein erheblicher Aufwand verbunden. Diese Aufgabe wäre von staatlichen Stellen wie dem Nachlassgericht kaum zu bewältigen. ${ }^{393}$ Dem Provider wiederum ist eine Kenntnisnahme der Nachrichteninhalte (telekommunikations-)rechtlich gar nicht gestattet. ${ }^{394}$ Die Änderung dieser Rechtslage mit der Absicht der Schaffung eines besseren postmortalen Persönlichkeitsschutzes zu fordern, hieße das Kind mit dem Bade auszuschütten. Folglich könnte allein die Annahme einer Infektion des gesamten Accounts durch einzelne höchstpersönliche Inhalte zu praktisch handhabbaren und eindeutigen Ergebnissen führen. Dies würde bei praxisnaher Betrachtung aber zu einer Unvererbbarkeit nahezu aller Nutzerkonten und damit zu einer grundsätzlichen Zurückstellung der Erbeninteressen führen. Denn in nahezu jedem Account dürften zumindest

391 BGH NJW 2018, 3178 Rn. 51; dem BGH insoweit zustimmend Seidler, NZFam 2020, 141, 143; bereits zuvor auch Kutscher, Der digitale Nachlass, S. 113 ff.; Kunz in: Staudinger, $\mathbb{S} 1922$ BGB Rn. 608; Bock, AcP (217) 2017, 370, 392 f.; Gloser, MittBayNot 2016, 12, 16.

392 Bräutigam in: DAV-Stellungnahme Nr.34/2013, S. 25 nennt das Beispiel von Liebesbriefen, die als Beweismittel in einem Prozess dienen; Seidler, Digitaler Nachlass, S. 111 f.; Gomille, ZUM 2018, 660, 664; Steiner/Holzer, ZEV 2015, 262, 263.

393 Herzog in: NK-Nachfolgerecht, Kap. 9 Rn. 45; dies. in: DAV-Stellungnahme Nr. 34/2013, S. 52 f.; Kutscher, Der digitale Nachlass, S. 113.

394 Herzog in: NK-Nachfolgerecht, Kap. 9 Rn. 45; Kunz in: Staudinger, $\mathbb{1} 1922$ BGB Rn. 608; Bock, AcP (217) 2017, 370, 392 f.; Gomille, ZUM 2018, 660, 664; Traschler, ZEuP 2020, 168, 173; zum Telekommunikationsrecht s. noch S. 148 ff. 
auch einige wenige private Inhalte gespeichert sein. ${ }^{395} \mathrm{Ob}$ ein solches $\mathrm{Er}-$ gebnis mit den gesetzlichen Wertungen des Erbrechts vereinbar ist, soll im nächsten Schritt geprüft werden.

\section{bb) Die Wertung der $\$ \$ 2047$ Abs. 2, 2373 BGB}

Dem Erbrecht selbst lässt sich in zwei Bestimmungen eine implizite Aussage über die Vererbbarkeit von (analogen) Rechtspositionen mit höchstpersönlichem Inhalt entnehmen. Die $\mathbb{S} \$ 2047$ Abs. 2, 2373 BGB regeln zwar nicht selbst die Vererbbarkeit höchstpersönlicher Inhalte, ${ }^{396}$ setzen diese aber voraus. So regelt $\$ 2047$ Abs. 2 BGB, dass „Schriftstücke, die sich auf die persönlichen Verhältnisse des Erblassers, auf dessen Familie oder auf den ganzen Nachlass beziehen," bei der Verteilung gemeinschaftlich bleiben. ${ }^{397}$ Dieser Anordnung bedarf es aber nur dann, wenn die genannten Schriftstücke überhaupt als Teil des Nachlasses den Erben zustehen. Nach $\$ 2373$ S. 2 BGB sind „Familienpapiere und Familienbilder“ beim Erbschaftskauf als im Zweifel nicht mitverkauft anzusehen. ${ }^{398}$ Gehörten etwa Familienbilder, die nach der Differenzierungstheorie wohl als höchstpersönlich zu bewerten und damit unvererbbar wären, nicht zur Erbmasse, liefe die Vorschrift des $\$ 2373$ S. 2 BGB prinzipiell leer. Daraus folgert die herrschende Meinung, dass die $\$ \$ 2047$ Abs. 2, 2373 BGB im Rückschluss die Vererbbarkeit höchstpersönlicher Rechtspositionen verlangen und dass das Gesetz nicht zwischen höchstpersönlichen und vermögenswerten Nachlassgegenständen unterscheidet. ${ }^{399}$

395 Das gilt insbesondere, wenn man wie Hoeren auch einfache Gruß-E-Mails zu den unvererblichen Inhalten zählen möchte, vgl. Hoeren, NJW 2005, 2113, 2114; Budzikiewicz, AcP (218) 2018, 558, 576; Gomille, ZUM 2018, 660, 664.

396 Insoweit ist KG Berlin, ZD 2017, 386 Rn. 62 zuzustimmen, das dann aber einen falschen Schluss zieht, vgl. Herzog in: NK-Nachfolgerecht, Kap. 9 Rn. 40a f.; dies., ZErb 2017, 205, 205 f.; differenzierend Alexander, notar 2017, 355, 356.

397 Dazu Eberl-Borges in: NK-BGB, $\$ 2047$ Rn. 4; Zimmer in: Prütting/Wegen/Weinrich (Hrsg.), $\$ 2047$ BGB Rn. 4 f.; Flechtner in: Burandt/Rojahn (Hrsg.), $\$ 2047$ BGB Rn. $4 \mathrm{ff}$.

398 Dazu Kroiß in: NK-BGB, $\$ 2373$ Rn. 2 f.; Gierl in: Burandt/Rojahn (Hrsg.), $\$ 2373$ BGB Rn. 4; Musielak in: MüKo BGB, $\$ 2373$ Rn. 5 f.

399 BGH NJW 2018, 3178 Rn. 49; Lieder in: Erman, $\mathbb{1 9 2 2}$ BGB Rn. 14a; ders./ Berneith, FamRZ 2016, 743, 743; Heintz/Ludyga, jM 2018, 398, 400; Gloser, MittBayNot 2016, 12, 16; Herzog in: NK-Nachfolgerecht, Kap. 9 Rn. 39 ff.; eingehend Herzog/Pruns, Der digitale Nachlass in der Vorsorge- und Erbrechtspraxis, S. 33 ff.; Seidler, Digitaler Nachlass, S. 122; Lange/Holtwiesche, ZErb 2016, 125, 
Indes lässt sich dem in diesen Normen ausgedrückten Rechtsgedanken nicht entnehmen, dass das Persönlichkeitsrecht dem Sukzessionsgedanken grundsätzlich untergeordnet wäre. Zum einen betreffen die \$S 2047 Abs. 2, 2373 BGB das Eigentum an Sachen im Sinne von $\$ 90$ BGB, welches nach dem Erbfall zwingend zugeordnet werden muss, um die Herrenlosigkeit der Gegenstände abzuwenden. ${ }^{400}$ Vor diese unerwünschte Alternative ist man aber im Falle des Nutzungsverhältnisses nicht zwangsläufig gestellt. ${ }^{401}$ Außerdem stammen beide Vorschriften aus dem Jahr 1900 und damit aus einer Zeit, bevor die Dogmatik des allgemeinen wie postmortalen Persönlichkeitsrechts entwickelt wurde. ${ }^{402}$ Mit Blick auf die Möglichkeiten der verfassungskonformen Auslegung (des Erbrechts) und der mittelbaren Drittwirkung der Grundrechte (in Nutzungsverträgen zwischen Privaten) kann den einfachrechtlichen \$S 2047 Abs. 2, 2373 BGB nach alledem nur eine eingeschränkte Aussagekraft über die Reichweite des Persönlichkeitsrechts beigemessen werden. ${ }^{403}$

\section{cc) Funktion und Reichweite der Wahrnehmungsberechtigung}

Die Differenzierungstheorie missversteht die Funktion und die Reichweite der Wahrnehmungsberechtigung nächster Angehöriger an dem postmortalen Persönlichkeitsrecht des Erblassers. Der postmortale Persönlichkeitsschutz des Erblassers wird von seinen nächsten Angehörigen treuhänderisch für ihn und in seinem Interesse ausgeübt. ${ }^{404}$ Daraus folgt, dass die Angehörigen gegen eine Verletzung des subjektlosen postmortalen Persönlichkeitsrechts mit Unterlassungs- und Beseitigungsansprüchen vorgehen

126; einschränkend Leipold in: MüKo BGB, $\$ 1922$ Rn. 34: Der Rechtsgedanke lasse sich „zunächst einmal auf die Vererbung des [Nutzungs-]Kontos“ übertragen.

400 So schon Budzikiewicz, AcP (218) 2018, 558, 575.

401 Das Nutzungsverhältnis könnte, wie die Gegenansicht nahelegt, auf die nächsten Angehörigen übergehen oder komplett erlöschen mit der Folge, dass auch die Accountinhalte zu löschen wären.

402 Bock, AcP (217) 2017, 370, 383 f.; Budzikiewicz, AcP (218) 2018, 558, 575 f.

403 So Bock, AcP (217) 2017, 370, 383 f., die den $\$ \$ 2047$ Abs. 2, 2373 BGB daher nur die Aussage regelmäßiger (nicht ausnahmsloser) Vererbbarkeit persönlicher Inhalte entnimmt; auf verfassungskonforme Auslegung und mittelbare Drittwirkung weist bereits hin Martini, JZ 2012, 1145, 1152.

404 Vgl. dazu bereits die Nachweise in Fn. 378 und 379 sowie ausführlich Schwab in: FS für Bengel und Reimann, S. 349 ff. 
können. ${ }^{405}$ Die Angehörigen werden hingegen nicht selbst Inhaber des Rechts. Die Wahrnehmungsbefugnis dient dazu, anstelle des Erblassers, der dazu selbst naturgemäß nicht mehr in der Lage ist, Persönlichkeitsrechtsverletzungen effektiv bekämpfen zu können. Die Wahrnehmungsbefugnis erweitert hingegen nicht den Kreis eigener subjektiver Rechte der Angehörigen und begründet keine zusätzliche parallele Rechtsnachfolge neben dem Erbrecht.

Bezogen auf die Accountinhalte ergibt sich daraus, dass die Angehörigen gegen den persönlichkeitsverletzenden Umgang etwa mit den privaten Nachrichten des Erblassers vorgehen können. ${ }^{406}$ Das schließt auch und gerade die Erben als Verpflichtete etwaiger Unterlassungsansprüche ein. ${ }^{407}$ Aus der Wahrnehmungsberechtigung lässt sich hingegen kein eigenes, dem Erbrecht vorrangiges Recht auf die und an den Inhalten selbst ableiten. ${ }^{408}$ Ein solches Recht wäre aber notwendig, um die aus der Differenzierungstheorie folgende Trennung zwischen vermögenswerten und höchstpersönlichen Inhalten durchzusetzen - etwa durch die Geltendmachung von entsprechenden Zugangsansprüchen gegen den Provider. Dem Vorschlag der Differenzierungstheorie, den Angehörigen die höchstpersönlichen Teile des Accountinhalts zuzuleiten, fehlt es daher an einer rechtlichen Grundlage. 409

405 Leipold, Erbrecht, Rn. 635; Schmitt in: Götting/Schertz/Seitz (Hrsg.), Handbuch Persönlichkeitsrecht, $\mathbb{2 9}$ Rn. 76; die Angehörigen haben hingegen keine Geldansprüche, vgl. BGH NJW 2006, 605, LS 1 und dazu Schmitt, a.a.O., Rn. $77 \mathrm{ff}$. m.w.N.

406 BGH NJW 2018, 3178 Rn. 53; Specht-Riemenschneider in: Röhricht/Graf von Westphalen/Haas (Hrsg.), HGB, Plattformnutzungsverträge, Rn. 92; Budzikiewicz, AcP (218) 2018, 558, 576; Bock, AcP (217) 2017, 370, 390; dies. in: Groll/ Steiner (Hrsg.), Praxishandbuch Erbrechtsberatung, Rn.20.32; Hoeren, MMR 2018, 749, 749.

407 Ebd. Fn. 406 sowie Preuß, NJW 2018, 3146, 3149; die Anspruchsgrundlage wäre $₫ 1004$ Abs. 1 BGB analog.

408 BGH NJW 2018, 3178 Rn. 53; Leipold in: MüKo BGB, $\mathbb{1} 1922$ Rn. 38; Specht-Riemenschneider, a.a.O. (Fn. 406); Herzog in: NK-Nachfolgerecht, Kap. 9 Rn. 50; hingegen ein Einsichtsrecht der Angehörigen unter Umständen anerkennend Seidler, Digitaler Nachlass, S. $102 \mathrm{f}$.

409 Daher vermag Hoeren, NJW 2005, 2113, 2114 nicht zu überzeugen; Herzog in: NK-Nachfolgerecht, Kap. 9 Rn. 51 f. m.w.N.; dies., ZErb 2017, 205, 207; Bräutigam in: Burandt/Rojahn (Hrsg.), nach $₫ 1922$ BGB Rn. 5; Bock in: Groll/Steiner (Hrsg.), Praxishandbuch Erbrechtsberatung, Rn. 20.32 f.; jüngst auch Hoeren, MMR 2018, 749, 749 f.: Es sei zwar „schade“, dass der BGH die vorgeschlagene Berücksichtigung des postmortalen Persönlichkeitsrechts abgelehnt habe, es handele sich aber um ein „dogmatisch profund begründetes, [...] herausragen- 
dd) Zwischenergebnis zu b)

Eine Differenzierung zwischen vermögenswerten und höchstpersönlichen Accountinhalten in der Form, dass letztere den nächsten Angehörigen des Erblassers zuzuleiten wären, ist abzulehnen. Gegen eine derartige Trennung sprechen die Wertung der $\int \$ 2047$ Abs. 2, 2373 BGB und die Dogmatik des postmortalen Persönlichkeitsrechts, aus der sich eine Spaltung des Nachlasses nicht ableiten lässt. Schließlich bedeutete die Vorstellung einer praktisch durchzuführenden Aufteilung der im Nutzungskonto enthaltenen Daten einen erheblichen Aufwand und große inhaltliche Abgrenzungsschwierigkeiten. Nach alledem ist festzuhalten, dass es nur einen einheitlichen (digitalen) Nachlass gibt, der sich aus vermögenswerten und höchstpersönlichen Bestandteilen zusammensetzt.

c) Kein Ausschluss der schlichten Kenntnisnahme durch die Erben

Wenn und soweit ein Aussortieren höchstpersönlicher Accountinhalte demnach weder rechtlich noch praktisch durchführbar ist, dann muss vor dem Hintergrund der bereits dargestellten Infektionsgefahr davon ausgegangen werden, dass die Erben mit dem Zugang zum Account (auch) Einblick in private und intime Informationen des Erblassers erhalten. Fraglich ist sodann, ob bereits diese schlichte Kenntnisnahme durch die Erben das postmortale Persönlichkeitsrecht des Erblassers verletzt und dadurch Abwehransprüche der Angehörigen zeitigt. ${ }^{410}$ Das setzt voraus, dass schon der nur lesende Zugriff auf die Accountinhalte einen Eingriff in die Individualität, Identität und Integrität des Menschen und den Achtungsanspruch des Verstorbenen darstellt. ${ }^{411}$ Hierbei kann unterschieden werden zwischen Privatnachrichten und Inhalten, welche der Erblasser öffentlich oder jedenfalls mit einem weiteren Personenkreis von Netzwerkkontakten („Freunden“) geteilt hat. In Bezug auf letztere ist der Erblasser insofern weniger schutzwürdig, als dass er einen Teil seiner Privatsphäre freiwillig

des Grundsatzurteil“; ders., MMR 2020, 183, 184 („,besonnenes und kluges Urteil“).

410 Für Handlungen jenseits der schlichten Kenntnisnahme wie bestimmte Formen von Veröffentlichung und Vermarktung oder der Diffamierung (mittels) privater und intimer Accountinhalte ist das unumstritten, vgl. die Nachweise in Fn. 406.

411 Zuck in: DAV-Stellungnahme Nr.34/2013, S. $84 \mathrm{f}$. mit Verweis auf BVerfG, NJW 2001, 2957, 2959; NJW 2006, 3409, 3409 f.; Bock, AcP (217) 2017, 370, 391. 
preisgegeben hat. ${ }^{412}$ Aber auch in Bezug auf die Privatnachrichten ist zu konstatieren, dass die Annahme einer Verletzung des postmortalen Persönlichkeitsrechts hier zu einer Aushöhlung des Erbrechts führen würde, die der Erbrechtsgarantie aus Art. 14 Abs. 1 S. 1 Var. 2 GG zuwiderläuft. Der personale Schutzbereich der Erbrechtsgarantie erstreckt sich nach ständiger Rechtsprechung des BVerfG „gerade, wenn nicht sogar in erster Linie auf den Erblasser." 413 Der Impetus, den Erblasser vor seinen Erben zu schützen, könnte sich dadurch negativ auf die Interessen des Erblassers selbst auswirken.

Überhaupt erscheint es fragwürdig, den Erben pauschal die Ausforschung der Persönlichkeit des Erblassers in Bezug auf intime Details der Lebensführung zu unterstellen. ${ }^{414}$ Entweder sind die Erben kraft gewillkürter Erbfolge - also auf ausdrücklichen Wunsch des Erblassers - in die Position des Rechtsnachfolgers gekommen. Oder sie haben diese Stellung der gesetzlichen Erbfolge zu verdanken, die auf dem Ehegatten- und Verwandtenerbrecht basiert und als normative Ordnung die Gerechtigkeitsvorstellung des Gesetzgebers ausdrückt. ${ }^{415}$ Die Erben sind dem Erblasser folglich in der Regel persönlich bekannt. Diese Tatsache ist sodann vor dem Hintergrund zu betrachten, dass der Erblasser zu Lebzeiten den postmortalen Umgang mit dem digitalen Nachlass durch testamentarische Anordnung, faktische Handlungen oder vertragliche Vereinbarung mit dem Provider durchaus selbst beeinflussen kann. ${ }^{416}$ Wenn der Erblasser nun keinerlei Vorkehrung gegen den Zugriff seiner ihm bekannten Erben auf die Accountinhalte trifft, spricht das dagegen, ein derart starkes und folgenreiches Schutzbedürfnis vor dem schlicht lesenden Zugriff auf die Daten anzunehmen. ${ }^{417}$

412 Kutscher, Der digitale Nachlass, S. 109 f.; Martini, JZ 2012, 1145, 1152; Lange/ Holtwiesche, ZErb 2016, 157, 160.

413 BVerfG NJW 2011, 366 Rn. 17; BVerfG NJW 1995, 2977, 2978; Axer in: BeckOK Grundgesetz, Art. 14 GG Rn. 146; Bock, AcP (217) 2017, 370, 392.

414 So aber Martini, JZ 2012, 1145, 1150; a.A. Bock, AcP (217) 2017, 370, 393.

415 Röthel, Erbrecht, Kap. 2 Rn. 5 ff.; ausführlich dies., Solidaritätskonzept und Statusorientierung des Erbrechts, in: Lipp/Röthel/Windel (Hrsg.), Familienrechtlicher Status und Solidarität, S. 97 ff.; a.A., die gesetzliche Erbfolge bilde den mutmaßlichen Willen des Erblassers ab, Muscheler, Erbrecht. Band I, Rn. $1276 \mathrm{ff}$. m.w.N.

416 Bock, AcP (217) 2017, 370, 393 f.; zu den Gestaltungsmöglichkeiten der Parteien des Nutzungsvertrags vgl. noch S. $159 \mathrm{ff}$.

417 Budzikiewicz, AcP (218) 2018, 576 f.; Herzog in: NK-Nachfolgerecht, Kap. 9 Rn. 34 und 50; Leipold in: MüKo BGB, $\mathbb{1} 1922$ Rn. 38; Specht-Riemenschneider in: 
d) Zwischenergebnis zu 2.

Das Merkmal der Höchstpersönlichkeit steht der Vererbbarkeit des Nutzungsvertrags im Verhältnis zwischen dem Erblasser, seinen nächsten Angehörigen und den Erben nicht entgegen. Die Erben rücken in das Nutzungsverhältnis des Erblassers mit dem Provider ein und erhalten dadurch Zugriff auf das Nutzungskonto und die darin enthaltenen Daten. Dieser Zugriff verletzt nicht per se das postmortale Persönlichkeitsrecht des Erblassers und kann daher auch nicht von den nächsten Angehörigen mit Unterlassungsansprüchen unterbunden werden. Die Accountinhalte werden vor dem Zugriff der Erben keiner Filterung unterzogen und nicht in vermögenswerte und höchstpersönliche Daten unterteilt. Die wahrnehmungsberechtigten Angehörigen können allenfalls dann Ansprüche wegen der Verletzung des postmortalen Persönlichkeitsrechts des Erblassers geltend machen, wenn Dritte oder die Erben mit den Accountinhalten in verletzender Weise umgehen. Denn aus dem Zugang zum Account folgt nicht etwa ein Recht der Erben zum beliebigen Umgang mit den Inhalten. ${ }^{418}$ Den Angehörigen stehen hingegen keine eigenen subjektiven Rechte an dem oder einem Teil des Nutzungsvertrags zu.

\section{Zwischenergebnis zu II.}

Im Regelfall ist der Vertrag zwischen einem Nutzer und dem Anbieter eines Sozialen Netzwerks vererbbar. Das Merkmal der Höchstpersönlichkeit als Grenze der Vererbbarkeit steht dem weder bezogen auf das Gefüge zwischen dem Erblasser, seinen Kommunikationspartnern und dem Provider noch im Verhältnis des Erblassers zu seinen Erben und nächsten Angehörigen grundsätzlich entgegen. Insofern besteht regelmäßig ein Gleichlauf zwischen analogen und digitalen Erbrechtsobjekten mit Persönlichkeitsbezug. Allerdings kann dieses Ergebnis nicht ungeprüft auf jedes Angebot von Informations- und Kommunikationsdiensten übertragen werden. Die genaue Funktionsweise des jeweiligen Internetdienstes und die darauf ausgerichteten Gewohnheiten und Erwartungen der Nutzer sind zu unter-

Röhricht/Graf von Westphalen/Haas (Hrsg.), HGB, Plattformnutzungsverträge Rn. 92; in diese Richtung auch Sorge, MMR 2018, 372, 377 a.E.

418 Leipold in: MüKo BGB, $\mathbb{S} 1922$ Rn. 38; ebenso Specht-Riemenschneider in: Röhricht/Graf von Westphalen/Haas (Hrsg.), HGB, Plattformnutzungsverträge Rn. 92. 
suchen. Das gilt auch und gerade in Ansehung der weiterhin zu erwartenden rasanten Entwicklung des Angebots, die Ausnahmen vom Grundsatz der Vererbbarkeit durchaus möglich machen kann. Dabei sind die (persönlichkeitsrechtlichen) Interessen des Erblassers durch den Rechtsübergang nach hier vertretener Auffassung in einem geringeren Maße gefährdet, als es die schwerpunktmäßige Behandlung dieses Aspekts in der bisherigen Debatte vermuten lässt. Das Persönlichkeitsrecht der Kommunikationspartner des Erblassers ist hingegen schon aktuell berührt und bedarf besonderer Aufmerksamkeit bei der Beobachtung technisch-funktionaler Entwicklungen der Internetdienste. Im Übrigen ist auf der nun folgenden Ebene - namentlich im Rahmen des Datenschutzrechts - nach Beschränkungen des bereits auf die Erben übergegangenen Rechts zu suchen, die unter anderem ebendiesen Interessen der Kommunikationspartner oder anderer Dritter dienen können.

\section{Beschränkung des übergegangenen Rechts}

Wenn die Erben als neue Accountinhaber und Parteien des Nutzungsvertrags die auf sie im Wege des Erbgangs übergegangenen Rechte geltend machen, können dieser Geltendmachung nach allgemeinen zivilrechtlichen Grundsätzen verschiedene Durchsetzungshindernisse entgegenstehen. ${ }^{419}$ Untersagt etwa das Datenschutzrecht der DS-GVO (dazu 1.) dem Provider die Verarbeitung der Accountdaten in Form der (umfassenden) Zugangsgewährung gegenüber den Erben, löst dies zivilrechtlich eine rechtliche Unmöglichkeit im Sinne des $\$ 275$ Abs. 1 BGB aus. ${ }^{420}$ Die vertragliche Leistungspflicht des Providers erlöscht in diesem Fall ipso iure, ${ }^{421}$ sodass der Anspruch der Erben auf Zugang dauerhaft ausgeschlossen wäre.

419 Kunz in: Staudinger, $\mathbb{S} 1922$ BGB Rn. $626 \mathrm{ff}$. spricht von „Zugangssperren“; nicht ganz eindeutig zur dogmatischen Einordnung Bock, AcP (217) 2017, 370, 397.

420 Gomille, ZUM 2018, 660, 664 f.; Martini/Kienle, JZ 2019, 235, 237 mit Verweis auf BGH NJW 2014, 2651 Rn.9 (zu $\$ 12$ Abs. 2 TMG) wonach der Mangel einer gesetzlich "erforderlichen datenschutzrechtlichen Ermächtigungsgrundlage" zu rechtlicher Unmöglichkeit gem. $\$ 275$ Abs. 1 BGB führt; Kunz in: Staudinger,

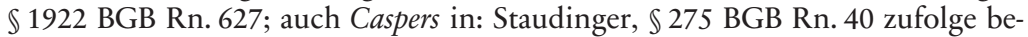
gründen gesetzliche Verbote mit Erlaubnisvorbehalt rechtliche Unmöglichkeit; zu rechtlicher Unmöglichkeit allgemein Ernst in: MüKo BGB, $\$ 275$ Rn. 41 ff.

421 Vgl. Lorenz in: BeckOK BGB, $\$ 275$ Rn. 65 sowie zu den sonstigen Rechtsfolgen in den Rn. 67 ff.; Grüneberg in: Palandt, $\$ 275$ BGB Rn. 31. 
Telekommunikationsrechtlich gilt dasselbe entsprechend, wenn und soweit der Anbieter dem sogenannten Kenntnisnahmeverbot aus $₫ 88$ Abs. 3 S. 1 TKG mit Bezug auf die Erben unterliegt (dazu 2.). ${ }^{422}$

\section{Das Datenschutzrecht der EU-Datenschutz-Grundverordnung}

Im Datenschutzrecht ist vornehmlich die seit dem 25. Mai 2018 in den Mitgliedstaaten gem. Art. 288 Abs. 2 S. 2 AEUV unmittelbar geltende DSGVO zu beachten. ${ }^{423}$ Zunächst ist zu prüfen, ob der Anwendungsbereich der DS-GVO bei den hier aufgeworfenen Fragen der Rechtsnachfolge in Nutzungsverträge überhaupt eröffnet ist (dazu a). Sofern der Anwendungsbereich eröffnet ist, genießt die DS-GVO als unionaler Sekundärrechtsakt Anwendungsvorrang vor dem nationalen Recht der Mitgliedstaaten, wenn und soweit dem nationalen Gesetzgeber keine Gestaltungsoptionen (insbesondere durch sogenannte Öffnungsklauseln) eingeräumt sind. ${ }^{424}$ Insoweit kommt $₫ 1$ Abs. 5 BDSG eine klarstellende Funktion zu. ${ }^{425}$ Sodann ist zu prüfen, ob es für den Provider datenschutzrechtlich zulässig ist, den Erben Zugang zum Nutzungskonto des Erblassers zu verschaffen. Das ist in Abhängigkeit von den Accountinhalten vor allem am Maßstab von Art. 6 DSGVO (dazu b) bzw. von Art. 9 DS-GVO (dazu c) zu messen.

422 KG Berlin, ZD 2017, 386 Rn. 66, 104 (mit kritischer Anm. von Klages) lässt die Vererbbarkeit eines Facebook-Accounts sogar explizit dahinstehen, da dem $\mathrm{Zu}$ gang ohnehin die „Durchsetzungssperre“ aus $\$ 88$ Abs. 3 TKG entgegenstehe.

423 Vgl. Art. 99 Abs. 2 DS-GVO; allgemein zum zweijährigen Übergangszeitraum vgl. Pauly in: Paal/Pauly (Hrsg.), Art. 99 DS-GVO Rn. 2 ff.; zum Übergangszeitraum mit Bezug auf das Facebook-Verfahren Martini/Kienle, JZ 2019, 235, 237 und BGH NJW 2018, 3178 Rn. 65 f.

424 Paal/Pauly in: Paal/Pauly (Hrsg.), DS-GVO/BDSG, Einleitung Rn. 20 f.; Kübling/ Klar/Sackmann, Datenschutzrecht, Rn. 60; Kühling, NJW 2017, 1985, 1986 f.; allgemein zum Anwendungsvorrang des Europarechts vgl. Oppermann/Classen/ Nettesheim, Europarecht. Ein Studienbuch, $\$ 10$ passim; Haratsch/Koenig/Pechstein, Europarecht, Rn. $196 \mathrm{ff}$. mit ausführlichen Nachweisen zur Rechtsprechung des EuGH.

425 Vgl. Ernst in: Paal/Pauly (Hrsg.), $\$ 1$ BDSG Rn. 13; Klar in: Kühling/Buchner (Hrsg.), 11 BDSG Rn. 31; Kühling, NJW 2017, 1985, 1986 f. 


\section{a) Anwendungsbereich der DS-GVO}

Der sachliche Anwendungsbereich der DS-GVO umfasst (vor allem) „die ganz oder teilweise automatisierte Verarbeitung personenbezogener Daten [...]“, vgl. Art. 2 Abs. 1 DS-GVO. Hierdurch sind mit der „Verarbeitung“ einerseits und mit „personenbezogenen Daten“ andererseits zwei zentrale Begriffe der DS-GVO als Tatbestandsmerkmale der Eröffnung des Anwendungsbereichs benannt.

Die Zugangsgewährung gegenüber den Erben durch den Provider erfüllt unproblematisch den weiten Verarbeitungsbegriff der DS-GVO, der in Art. 4 Nr. 2 DS-GVO legal definiert wird und jeden Vorgang im Zusammenhang mit personenbezogenen Daten wie u.a. „[...] das Abfragen, die Verwendung, die Offenlegung durch Übermittlung, Verbreitung oder eine andere Form der Bereitstellung" umfasst. ${ }^{426}$ Das gilt unabhängig davon, ob die Erben sich mit den ihnen bekannten Login-Daten des Erblassers nach dem Erbfall weiterhin im Erblasseraccount anmelden oder ob der Provider diese Anmeldung erst durch die Mitteilung oder Zurücksetzung des Passworts ermöglicht. Denn die „Bereitstellung“ als Verarbeitungsvariante im Sinne des Art. 4 Nr. 2 DS-GVO ist auch dann erfüllt, wenn die verantwortliche Stelle die Daten zweckgerichtet bereitstellt und die eigentliche zugreifende Aktivität von Dritten (i.e. den Erben) ausgeht. ${ }^{427}$

Näherer Betrachtung bedarf das zentrale Schutzobjekt der DS-GVO, sprich das personenbezogene Datum, hinter dem nach Art. 4 Nr. 1 DSGVO die betroffene Person steht. ${ }^{428}$ Als betroffene Personen kommen hier der Erblasser (dazu aa) und seine Kommunikationspartner (dazu bb) in Betracht.

Schließlich darf kein privilegierender Ausnahmetatbestand vom Anwendungsbereich der DS-GVO einschlägig sein (dazu cc). Teilweise wird im hier relevanten Zusammenhang erwogen, insoweit Art. 2 lit.c) DS-GVO

$426 \mathrm{Zu}$ dem weiten Begriff der „Verarbeitung“ vgl. Ernst in: Paal/Pauly (Hrsg.), Art. 4 DS-GVO Rn. 20 ff.; Schild in: BeckOK Datenschutzrecht, Art. 4 DS-GVO Rn. 29 ff.; Arning/Rothkegel in: Taeger/Gabel (Hrsg.), Art. 4 DS-GVO Rn. 56 ff.; Klabunde in: Ehmann/Selmayr (Hrsg.), Art. 4 DS-GVO Rn. 22 ff.

427 Vgl. Schild in: BeckOK Datenschutzrecht, Art. 4 DS-GVO Rn. 50; ähnlich Reimer in: HK-DS-GVO, Art. 4 Rn. 69 f., der den Begriff der „Bereitstellung“ mit Bezug auf die vormalige Regelung des $₫ 3$ Abs. 4 Nr. 3 lit. a) BDSG a.F. bestimmt.

428 Vgl. Kühling/Klar/Sackmann, Datenschutzrecht, Rn. 248; Ziebarth in: HK-DSGVO, Art. 4 Rn. 7 und 9. 
(„Verarbeitung personenbezogener Daten [...] zur Ausübung ausschließlich persönlicher oder familiärer Tätigkeiten“) heranzuziehen. ${ }^{429}$

\section{aa) Kein postmortaler Datenschutz des Erblassers}

Nach Art. 4 Nr. 1 DS-GVO sind personenbezogene Daten „Informationen, die sich auf eine identifizierte oder identifizierbare natürliche Person beziehen." Bei praxisnaher Betrachtung ist davon auszugehen, dass der Account des Erblassers (auch) Informationen enthält, die sich auf den Erblasser selbst beziehen. Der potenzielle Zugriff auf diese Informationen als datenschutzrechtlich erhebliche Handlung findet aber erst nach dem Tod des Erblassers als ursprünglichem Accountinhaber statt.

Der Begriff der natürlichen Person aus Art. 4 Nr. 1 DS-GVO ist in Verbindung mit Erwägungsgrund 27 S. $1^{430}$ als eindeutiger Hinweis darauf zu sehen, dass es sich bei der betroffenen Person um einen lebenden Menschen handeln muss. ${ }^{431}$ Die DS-GVO reguliert die Verarbeitung personenbezogener Daten von Verstorbenen nicht. ${ }^{432}$ Der Kenntnisnahme der Accountinhalte steht de lege lata mithin kein postmortales Datenschutzrecht des Erblassers entgegen. ${ }^{433}$ Dieses Ergebnis steht in Einklang mit der nationalen und der europäischen Grundrechtsbasis des Datenschutzrechts: Auf nationaler Ebene sind Verstorbene vom persönlichen Schutzbereich des

429 Dies (wohl) bejahend Heintz/Ludyga, jM 2018, 398, 402; a.A. Martini/Kienle, JZ 2019, 235, 237. Ausführlich dazu sogleich auf S. $123 \mathrm{f}$.

430 Erwägungsgrund 27 der DS-GVO lautet: „Diese Verordnung gilt nicht für die personenbezogenen Daten Verstorbener. Die Mitgliedstaaten können Vorschriften für die Verarbeitung der personenbezogenen Daten Verstorbener vorsehen.“ Zur rechtspolitischen Empfehlung an den nationalen Gesetzgeber von dieser Gestaltungsmöglichkeit Gebrauch zu machen, vgl. Gutachten der Datenethikkommission vom 23.10.2019, S. 111, abrufbar a.a.O. (Fn. 30).

431 Ernst in: Paal/Pauly (Hrsg.), Art. 4 DS-GVO Rn. 4; Gola in: Gola (Hrsg.), Art. 4 DS-GVO Rn. 26 ff.; Arning/Rothkegel in: Taeger/Gabel (Hrsg.), Art. 4 DS-GVO Rn. 18 ff.; Kühling/Klar/Sackmann, Datenschutzrecht, Rn. 250; im Ergebnis auch BGH NJW 2018, 3178 Rn. 67.; a.A. zur Rechtslage vor Geltung der DS-GVO Culmsee, Postmortaler Datenschutz, in: Taeger (Hrsg.), Tagungsband DSRIHerbstakademie 2013, S. $413 \mathrm{ff}$.

432 Sonderfälle, in denen Daten Verstorbener auch Lebende betreffen (z.B. Informationen zu Erbkrankheiten des Toten), mögen vereinzelt eine Ausnahme begründen, vgl. Kühling/Klar/Sackmann, Datenschutzrecht, Rn. 250; Arning/Rothkegel in: Taeger/Gabel (Hrsg.), Art. 4 DS-GVO Rn. 18 m.w.N.

433 Martini/Kienle, JZ 2019, 235, 237 finden dieses Ergebnis „kritikwürdig“; zur rechtspolitischen Diskussion vgl. den Nachweis in Fn. 430. 
sich aus dem allgemeinen Persönlichkeitsrecht ableitenden Rechts auf informationelle Selbstbestimmung (Art. 2 Abs. 1 in Verbindung mit Art. 1 Abs. 1 GG) ausgeschlossen. ${ }^{434}$ Auf europäischer Ebene gilt grundsätzlich, dass die Grundrechtsberechtigung aus der EU-Grundrechte-Charta ${ }^{435}$ sowie aus der EMRK ${ }^{436}$ ebenfalls mit dem Tod des Grundrechtsträgers endet.

Nach alledem lässt sich die Eröffnung des Schutzbereichs der DS-GVO nicht mit dem Erblasser als „betroffener Person“ im Sinne von Art. 4 Nr. 1 DS-GVO begründen.

bb) Kommunikationspartner des Erblassers als „betroffene Person“

Als betroffene Person im Sinne des Art. 4 Nr. 1 DS-GVO kommen ferner die Kommunikationspartner des Erblassers in Betracht. Die Privatnachrichten, die im Account des Erblassers vorgehalten werden, sind mit dem Profilnamen der Kommunikationspartner bezeichnet und mit dem entsprechenden Profil verlinkt. Den zugreifenden Erben ist es daher möglich, den Profilnamen und -inhalt gemeinsam mit den Nachrichteninhalten zu betrachten. Dieser Zusammenschau lassen sich ohne Weiteres Informationen entnehmen, welche sich auf die Kommunikationspartner als identifizierte oder identifizierbare natürliche Person beziehen. Damit liegen personenbezogene Daten im Sinne von Art. 4 Nr. 1 DS-GVO vor.

Trotzdem soll der Anwendungsbereich nach einer Ansicht auch mit Blick auf die Kommunikationspartner des Erblassers nicht eröffnet sein. ${ }^{437}$

434 Gersdorf in: BeckOK Informations- und Medienrecht, Art. 2 GG Rn. 16 und 31; Di Fabio in: Maunz/Dürig (Begr.), Art. 2 Abs. 1 GG Rn. 173, 226; kritisch zur rein „richterlichen Rechtsfortbildung [ohne] textliche Basis“ für die Bestimmung der Grundrechtsträgerschaft Ipsen, Staatsrecht II, Grundrechte, Rn. 317 f.; zum postmortalen Persönlichkeitsrecht, das sich allein aus Art. 1 Abs. 1 GG ableitet, vgl. bereits die Nachweise in Fn. 375, 376 und 389.

435 Jarass/Kment, EU-Grundrechte, $\$ 4$ Rn. 29; zum postmortalen Würdeschutz aus Art. 1 S. 1 EU-GRCh vgl. Callies in: Callies/Ruffert (Hrsg.), EUV/AEUV, Art. 1 EU-GRCh Rn. 18 m.w.N. Die DS-GVO nimmt in ihrem Erwägungsgrund Nr. 1 explizit Bezug auf Art. 8 Abs. 1 EU-GRCh.

436 Grabenwarter/Pabel, Europäische Menschenrechtskonvention, $\mathbb{1} 17$ Rn.4; Grabenwarter, European Convention on Human Rights, Art. 1 Rn. 3; Meyer-Ladewig/Nettesheim in: HK-EMRK, Art. 1 EMRK Rn. 24. Potenziell einschlägig wäre vorliegend Art. 8 Abs. 1 EMRK.

437 So der Bericht der Arbeitsgruppe „Digitaler Neustart“ der Konferenz der Justizministerinnen und Justizminister der Länder, S. 347 f., abrufbar a.a.O. (Fn. 43) mit irreführendem Verweis auf Kühling/Martini u.a., Die DSGVO und das natio- 
Dies wird mit dem unmittelbaren Zusammenhang zwischen der Datenverarbeitung und dem Erbfall und der daraus folgenden Nähe zum postmortalen Datenschutz begründet: Die in Erwägungsgrund $27^{438}$ ausgedrückte (Selbst-)Begrenzung des Anwendungsbereichs der DS-GVO werde unterlaufen, wenn die Daten des Verstorbenen wegen der parallelen Betroffenheit der Kommunikationspartner doch in den Anwendungsbereich der DS-GVO fielen. ${ }^{439}$ Im Umkehrschluss sei ein umfassender Anwendungsausschluss der DS-GVO bezüglich der Zugriffsrechte der Erben auf alle Kommunikationsdaten des Erblassers anzunehmen. ${ }^{440}$

Diese Ansicht überzeugt nicht. ${ }^{441}$ Die DS-GVO trägt ausweislich ihres Erwägungsgrunds 1 der Grundrechtsposition der betroffenen Person Rechnung. Die lebenden Kommunikationspartner sind Grundrechtsträger und betroffene Personen im Sinne der DS-GVO. Dieser Befund darf nicht negiert und unterlaufen werden. Die Feststellung, dass die DS-GVO den postmortalen Datenschutz nicht erfasst, ist als Ausnahme vom Anwendungsbereich grundsätzlich eng auszulegen. Um der Gegenansicht gerecht zu werden, müsste die Ausnahme aber sogar extensiv über ihren eigenen Wortlaut hinaus auch auf Personen bezogen werden, die (wie die Kommunikationspartner) vom Wortlaut des Art. 4 Nr. 1 DS-GVO erfasst sind. Das diese Lesart nicht überzeugen kann, zeigt ein Blick auf die Diskussion um solche Daten, die sich zwar auf den Verstorbenen beziehen, aber - wie etwa Informationen zu Erbkrankheiten - auf die Nachkommen als lebende Personen durchschlagen. ${ }^{442}$ Wenn solche Daten gleichsam mittelbar in den Anwendungsbereich der DS-GVO fallen, dann muss das erst recht für die unmittelbar erfasste Korrespondenz zwischen dem Erblasser und seinen Kommunikationspartnern gelten.

nale Recht, S. 21; a.A. Wüsthof, ErbR 2018, 579, 582; unklar Schneider, Datenschutz nach der EU-Datenschutz-Grundverordnung, S. 103 f.; ausdrücklich offen gelassen in BGH NJW 2018, 3178 Rn. 70.

438 Vgl. den Wortlaut von Erwägungsgrund 27 in Fn. 430.

439 Bericht der Arbeitsgruppe „Digitaler Neustart“ der Konferenz der Justizministerinnen und Justizminister der Länder, S. 347 f., abrufbar a.a.O. (Fn. 43).

440 Ebd. Fn. 439.

441 Wie hier Martini/Kienle, JZ 2019, 235, 237; bezogen auf die grundsätzliche Eröffnung des Anwendungsbereichs der DS-GVO auch Wüsthof, ErbR 2018, 579, 582.

442 Vgl. die Nachweise in Fn. 432 sowie Klar/Kühling in: Kühling/Buchner (Hrsg.), Art. 4 Nr. 1 DS-GVO Rn. 5 und Schantz/Wolff, Das neue Datenschutzrecht, Rn. 320. 
Die Zugangsgewährung der Provider gegenüber den Erben ist folglich eine Verarbeitung personenbezogener Daten und fällt damit in den Anwendungsbereich der DS-GVO.

cc) Ausschluss des Anwendungsbereichs gem. Art. 2 Abs. 2 DS-GVO

Ferner wird an der Eröffnung des Anwendungsbereichs unter Bezugnahme auf Art. 2 Abs. 2 lit.c) DS-GVO gezweifelt. ${ }^{443}$ Danach findet die DSGVO „keine Anwendung auf die Verarbeitung personenbezogener Daten durch natürliche Personen zur Ausübung ausschließlich persönlicher oder familiärer Tätigkeiten. “ Zwar mögen einige Nutzungsverhältnisse vorrangig oder sogar allein persönlichen oder familiären Zwecken dienen. Erwägungsgrund 18 S. 2 erwähnt sogar explizit die „Nutzung sozialer Netze“ als mögliches Beispiel einer persönlichen oder familiären Tätigkeit. Zu beachten ist aber, dass die Zugangsgewährung zum Erblasseraccount als datenschutzrechtlich relevanter Verarbeitungsvorgang nicht durch eine natürliche Person zu den genannten Zwecken erfolgt, wie es Art. 2 Abs. 2 lit.c) DS-GVO voraussetzt. Im Gegenteil verarbeitet der Provider die personenbezogenen Daten gerade im Rahmen seiner wirtschaftlichen Tätigkeit. Konsequenterweise lautet Erwägungsgrund 18 S. 3: „Diese Verordnung gilt jedoch für die Verantwortlichen [...], die die Instrumente für die Verarbeitung personenbezogener Daten für solche persönlichen oder familiären Tätigkeiten bereitstellen." Weder eine Privilegierung der Providertätigkeit noch die Schutzlosigkeit der datenschutzrechtlichen Interessen der Kommunikationspartner wäre mit dem Sinn und Zweck der DS-GVO vereinbar. Ein Anwendungsausschluss gem. Art. 2 Abs. 2 lit.c) DS-GVO kommt mithin nicht in Betracht. ${ }^{444}$

443 In diese Richtung Heintz/Ludyga, jM 2018, 398, 402; zum alten Recht gem. $\$ \$ 1$ Abs. 2 Nr. 3, 27 Abs. 1 S. 2 BDSG a.F. auch Bock, AcP 217 (2017), 370, 399 f.

444 Martini/Kienle, JZ 2019, 235, 237; Kühling/Raab in: Kühling/Buchner (Hrsg.), Art. 2 DS-GVO Rn. 25; Zerdick in: Ehmann/Selmayr (Hrsg.), Art. 2 DS-GVO Rn. 11; ebenso Ernst in: Paal/Pauly (Hrsg.), Art. 2 DS-GVO Rn. 20 f., nach dem Art. 2 Abs. 2 lit. c) DS-GVO als Ausnahmenorm grundsätzlich restriktiv auszulegen ist. 
dd) Zwischenergebnis zu a)

Der Anwendungsbereich der DS-GVO ist in Bezug auf die Kommunikationspartner des Erblassers als betroffene Personen im Sinne von Art. 4 Nr. 1 DS-GVO eröffnet. Die Zugangsgewährung durch die Provider gegenüber den Erben steht damit unter dem Grundverordnungsregime und unterfällt grundsätzlich dem präventiven Verbot mit Erlaubnisvorbehalt des Art. 6 Abs. 1 S. 1 DS-GVO. ${ }^{445}$ Der nutzungsvertragliche Zugangsanspruch der Erben ist in der Folge nur soweit durchsetzbar, wie seine Erfüllung durch den Provider mit der DS-GVO in Einklang steht.

\section{b) Erlaubnistatbestände des Art. 6 Abs. 1 DS-GVO}

Art. 6 Abs. 1 S. 1 DS-GVO statuiert ein präventives Verbot der Datenverarbeitung mit Erlaubnisvorbehalt. ${ }^{446}$ Die Zugangsgewährung durch den Provider ist demnach unzulässig, wenn und soweit nicht eine Verarbeitungserlaubnis einschlägig ist. Als Erlaubnistatbestände kommen hier die Einwilligung der Kommunikationspartner in die Verarbeitung der sie betreffenden personenbezogenen Daten, Art. 6 Abs. 1 S. 1 lit. a DS-GVO (dazu aa), die Vertragserfüllung gem. Art. 6 Abs. 1 S. 1 lit. b) Var. 1 DS-GVO (da$\mathrm{zu} \mathrm{bb)} \mathrm{sowie} \mathrm{die} \mathrm{Wahrung} \mathrm{berechtigter} \mathrm{Interessen} \mathrm{im} \mathrm{Sinne} \mathrm{von} \mathrm{Art.} 6$ Abs. 1 S. 1 lit. f) DS-GVO (dazu cc) infrage. ${ }^{447}$

aa) Einwilligung, Art. 6 Abs. 1 S. 1 lit. a) DS-GVO

Der Einwilligung kommt als Erlaubnistatbestand nach der Konzeption des europäischen Datenschutzgesetzgebers insgesamt eine hervorgehobene Be-

445 Das gilt gem. Art. 95 DS-GVO und Erwägungsgrund 173 S. 1, soweit nicht die ePrivacy-RL Sonderregelungen trifft, vgl. Martini/Kienle, JZ 2019, 235, Fn. 37 mit Verweis auf Heun in: Auernhammer, $\mathbb{} 88$ TKG Rn. 4, 67 ff.; vgl. dazu Kiparski/Sassenberg, CR 2018, 324, $324 \mathrm{ff}$. sowie ausführlich Bulowski, Regulierung von Internetkommunikationsdiensten, S. $101 \mathrm{ff}$.

446 Frenzel in: Paal/Pauly (Hrsg.), Art. 6 DS-GVO Rn. 1; ausführlich und differenzierend Taeger in: Taeger/Gabel (Hrsg.), Art. 6 DS-GVO Rn. 3 ff.; Kühling/Klar/Sackmann, Datenschutzrecht, Rn. 322; Heberlein in: Ehmann/Selmayr (Hrsg.), Art. 6 DS-GVO Rn. 1.

447 BGH NJW 2018, 3178 Rn. 70 prüft (nur) Art. 6 Abs. 1 S. 1 lit. b) Var. 1 sowie lit. f) DS-GVO. 
deutung zu. ${ }^{448}$ Die betroffene Person kann in die Verarbeitung der auf sie bezogenen Daten einwilligen und erhält dadurch sowie durch die Möglichkeit des Widerrufs der Einwilligung gem. Art. 7 Abs. 3 DS-GVO privatautonomen Einfluss auf die Rechtsmäßigkeit des Verarbeitungsvorgangs.

$\mathrm{Ob}$ und inwieweit eine wirksame Einwilligung der Kommunikationspartner in die Verarbeitung durch den Provider vorliegt, ist freilich eine Frage des Einzelfalls und kann deshalb nicht abstrakt beurteilt werden. Grundsätzlich ist allerdings zu beachten, dass Art. 6 Abs. 1 S. 1 lit. a) DSGVO die Einwilligung in die Datenverarbeitung „für einen oder mehrere bestimmte Zwecke“ voraussetzt. Das Merkmal der Bestimmtheit (in der englischen Sprachfassung: „specific“) lässt Zweifel daran aufkommen, dass die Zugangsgewährung gegenüber den Erben auf eine etwaige Einwilligung der Kommunikationspartner gestützt werden kann. Die Voraussetzungen der Bestimmtheit sowie der Informiertheit ${ }^{449}$ des Einwilligenden stehen in engem Zusammenhang zueinander und sollen der betroffenen Person in ihrem Zusammenspiel ermöglichen, die Tragweite ihrer Erklärung zu beurteilen. ${ }^{450}$ Auch wenn die Kommunikationspartner gegenüber dem Provider in die Datenverarbeitung zu manchen Zwecken eingewilligt haben sollten, wird dazu wohl kaum die Bereitstellung gegenüber einer gem. $\$ 2032$ Abs. 1 BGB unbestimmten Zahl an Erben gehören. ${ }^{451}$ Die Zugangsgewährung gegenüber den Erben wird demnach regelmäßig eine sogenannte Zweckänderung darstellen.

Der Provider unterliegt dem Zweckbindungsgrundsatz aus Art. 5 Abs. 1 lit.b) S. 1 HS 2 DS-GVO. 452 Daher darf der Provider die Datenverarbeitung im Falle einer Zweckänderung nur dann vornehmen, wenn der neue Zweck (i.e. die Erfüllung des Zugangsanspruchs der Erben) mit dem ur-

448 Buchner/Kühling, DuD 2017, 544, 544 ff.; Wendehorst/von Westphalen, NJW 2016, 3745, 3745; in diesem Sinne mit Nachdruck auch Frenzel in: Paal/Pauly (Hrsg.), Art. 7 DS-GVO Rn. 1; Kühling/Klar/Sackmann, Datenschutzrecht, Rn. 325; ausführlich zur Einwilligung allgemein Ernst ZD 2017, 110.

449 Diese Voraussetzung folgt aus Art. 4 Nr. 11 DS-GVO, dazu Ernst, ZD 2017, 110 , 113; Mantz in: HK-DS-GVO, Art. 4 Rn. 176; Radlanski, Das Konzept der Einwilligung in der datenschutzrechtlichen Realität, S.16f. m.w.N.; Tavanti, RDV 2016, 231, $234 \mathrm{f}$.

450 Vgl. nur Kühling/Klar/Sackmann, Datenschutzrecht, Rn. $504 \mathrm{ff}$.

451 Ähnlich Martini/Kienle, JZ 2019, 235, 240 allerdings zur Einwilligung im Sinne von Art. 9 Abs. 2 lit. a) DS-GVO. Siehe dazu noch auf S. $144 \mathrm{f}$.

452 Zum Grundsatz der Zweckbindung Mantz/Marosi in: Specht/Mantz (Hrsg.), Handbuch Europäisches und deutsches Datenschutzrecht, $\mathbb{S} 3$ Rn. 81 ff.; Reimer in: HK-DS-GVO, Art. 5 Rn. 18 ff; Roßnagel in: NK-Datenschutzrecht, Art. 5 DSGVO Rn. $63 \mathrm{ff}$. 
sprünglichen Zweck, in den die Kommunikationspartner als Betroffene eingewilligt haben, nicht unvereinbar ist. ${ }^{453}$ Ferner hat der Provider die zusätzlichen Voraussetzungen aus Art. 6 Abs. 4 DS-GVO zu beachten. ${ }^{454}$ Die damit aufgeworfenen Fragen können und sollen hier nicht im Einzelnen vertieft werden, weil die Argumentation stark von den Details des Einzelfalls abhängig ist. Festzustellen ist aber jedenfalls, dass nach Erwägungsgrund 50 unter anderem die vernünftigen Erwartungen der betroffenen Person hinsichtlich der Zweckänderung zu berücksichtigen sind. ${ }^{455}$ Insoweit kann auf die Ausführungen zur Erwartungshaltung der Nutzer im Rahmen der Höchstpersönlichkeit verwiesen werden: Während der BGH und Teile der Literatur davon ausgehen, dass der Übergang eines Nutzerkontos auf die Erben samt Zugriffsrecht zur vernünftigen Erwartung der Kommunikationspartner gehöre ${ }^{456}$, ist ein dahingehendes Bewusstsein nach hier vertretener Ansicht lebensfern und sollte nicht unterstellt werden. 457

Nach alledem bestehen zumindest Zweifel daran, dass die Zugangsgewährung gegenüber den Erben auf eine (zu anderen Zwecken erteilte) Einwilligung der Kommunikationspartner gestützt werden kann. Unbenommen bleibt es dem Provider freilich, eine Einwilligung zu ebendiesem Zweck bei den Kommunikationspartnern eigens einzuholen.

\section{bb) Erfüllung eines Vertrags, Art. 6 Abs. 1 S. 1 lit. b) Var. 1 DS-GVO}

Als weiterer Erlaubnistatbestand kommt Art. 6 Abs. 1 S. 1 lit. b) Var. 1 DSGVO in Betracht. Danach ist die Datenverarbeitung rechtmäßig, wenn sie „für die Erfüllung eines Vertrags, dessen Vertragspartei die betroffene Person ist, [...] erforderlich" ist. Der Tatbestand der Vertragserfüllung steht in engem Zusammenhang mit der Einwilligung, da die Verarbeitung der Daten - wenn auch nicht explizit adressiert - als erforderliches Zwischenziel

453 Außerdem löst die Verarbeitung zu einem anderen als dem ursprünglichen Zweck gem. Art. 13 Abs. 3 und 14 Abs. 4 DS-GVO eine Informationspflicht des Verantwortlichen gegenüber der betroffenen Person aus, vgl. Steinrötter/Rahimil Tran, EWS 2019, 301, 306.

454 Reimer in: HK-DS-GVO, Art. 5 Rn. 24 m.w.N.; Herbst in: Kühling/Buchner (Hrsg.), Art. 5 DS-GVO Rn. 45 ff.; Frenzel in: Paal/Pauly (Hrsg.), Art. 5 DS-GVO Rn. 30 f.; zu diesem sog. Kompatibilitätstest Schantz, NJW 2016, 1841, 1844.

455 Kühling/Klar/Sackmann, Datenschutzrecht, Rn. 424.

456 Vgl. dazu bereits die Nachweise in Fn. 331.

457 Siehe dazu S. $97 \mathrm{ff}$. 
gleichsam von der Freiwilligkeit des privatautonomen Vertragsschlusses mitumfasst ist. ${ }^{458}$ Der Wortlaut des Erlaubnistatbestands benennt zwei Voraussetzungen, denen hier nachzugehen ist. Da ist zum einen die Stellung der betroffenen Person als Vertragspartei (dazu (1)) und zum anderen die Erforderlichkeit der Datenverarbeitung zur Vertragserfüllung (dazu (2)).

\section{(1) Die betroffene Person als Vertragspartei}

Die betroffene Person muss Partei gerade desjenigen Vertrags sein, zu dessen Erfüllung die Verarbeitung erforderlich ist. ${ }^{459}$ In sozialen Netzwerken als Mehrparteienkonstellationen ist folglich zwischen unterschiedlichen Schuldverhältnissen zu unterscheiden. ${ }^{460}$ Das Vertragsverhältnis zwischen dem Provider und dem Erblasser, in welches die Erben einrücken, kann nicht als Anknüpfungspunkt für die Verarbeitung der auf die Kommunikationspartner bezogenen Daten herangezogen werden, weil die Kommunikationspartner insoweit keine Parteistellung innehaben. ${ }^{461} \mathrm{In}$ dem allein entscheidenden Vertragsverhältnis zwischen Provider und Kommunikationspartner schuldet der Betreiber des sozialen Netzwerks jedenfalls die Übermittlung der Nachrichten an das Konto des Empfängers. ${ }^{462}$ Der BGH geht darüber aber noch hinaus, wenn er nicht nur für die Übermittlung, sondern auch für den Zugang des Empfängers zu den Nachrichten auf den

458 Vgl. Reimer in: HK-DS-GVO, Art. 6 Rn. 18; Albers/Veit in: BeckOK Datenschutzrecht, Art. 6 DS-GVO Rn. 29; ähnlich auch Buchner/Petri in: Kühling/Buchner (Hrsg.), Art. 6 DS-GVO Rn. 26.

459 Ausführlich Britz/Indenhuck, Die Daten der Dritten - Verarbeitung drittbezogener Daten im Vertragsverhältnis, in: Taeger (Hrsg.), Tagungsband DSRI-Herbstakademie 2018, S. $231 \mathrm{ff}$.

460 Explizit zu sozialen Netzwerken Schantz in: NK-Datenschutzrecht, Art. 6 Abs. 1 DS-GVO Rn. 34 a.E. sowie allgemein Rn. 20; Martini/Kienle, JZ 2019, 235, 237.

461 Vgl. ebd. Fn. 460. Unschädlich ist es nach dem Wortlaut der Norm hingegen, wenn der Empfänger der Daten (= die Erben) selbst nicht Vertragspartei ist, vgl. dazu Martini/Kienle, JZ 2019, 235, 237; Kramer in: Auernhammer, Art. 6 DSGVO Rn. 37; a.A. wohl Schantz in: NK-Datenschutzrecht, Art. 6 Abs. 1 DS-GVO Rn. 20: Keine „Verträge zugunsten Dritter“.

462 Nach Martini/Kienle, JZ 2019, 235, 238 dort in Fn. 46 ist ein Vergleich mit analogen Briefen heranzuziehen, bei denen die Deutsche Post dem Absender (nur) die Übermittlung in das Postfach des Empfängers schulde. Der Zugang zum Postfach sei hingegen eine Frage des Vertrags zwischen Empfänger und der Deutschen Post. 
Vertrag zwischen dem Provider und dem Kommunikationspartner abstellt. ${ }^{463} \mathrm{Da}$ die Zustellungspflicht des Providers gegenüber den Kommunikationspartnern nicht personen- sondern kontobezogen sei, umfasse die Erfüllung dieser Pflicht die Übermittlung an das Nutzerkonto des Empfängers sowie die Ermöglichung des jederzeitigen Abrufs der Inhalte an dieser Stelle durch den Berechtigten. ${ }^{464}$ Der Wechsel der Person des Berechtigten qua Erbfall ändere an alledem nichts. ${ }^{465}$ Dieser Sichtweise des BGH wird vorgeworfen, nicht hinreichend trennscharf zwischen den grundsätzlich nur relativ wirkenden Schuldverhältnissen zu unterscheiden. ${ }^{466}$

Zwischen den beiden Sichtweisen entscheidend ist die Frage, inwieweit dem Erlaubnistatbestand aus lit. b) eine streng schuldrechtliche oder eine wertungsoffene Konzeption zugrunde liegt. Dieser Frage sollte sich sinnvollerweise über die zweite Voraussetzung aus Art. 6 Abs. 1 S. 1 lit.b) Var. 1 DS-GVO, dem Merkmal der Erforderlichkeit, weiter genähert werden. Denn lit. b) gestattet die Datenverarbeitung gerade, wenn und soweit sie zur Vertragserfüllung erforderlich ist. Insofern besteht eine Wechselbeziehung zwischen der Erforderlichkeit und dem vertraglichen Pflichtenregime. 467

(2) Erforderlichkeit i.S.v. Art. 6 Abs. 1 S. 1 lit. b) Var. 1 DS-GVO

$\mathrm{Zu}$ fragen ist, ob es im Sinne von Art. 6 Abs. 1 S. 1 lit. b) Var. 1 DS-GVO für die Erfüllung des Nutzungsvertrags zwischen den Kommunikationspartnern und dem Provider erforderlich ist, den Erben Zugang zum Account des Erblassers zu verschaffen.

Zur Auslegung des Merkmals der Erforderlichkeit werden dabei im Wesentlichen zwei Ansichten vertreten: Ein weiter Ansatz möchte unter Berücksichtigung der Interessen aller Beteiligten die Erforderlichkeit dann bejahen, wenn es keine zumutbare Alternative gleicher Eignung gibt, um

463 BGH NJW 2018, 3178 Rn. 72.

464 BGH NJW 2018, 3178 Rn. 73. Vgl. zu dieser technisch-formalen Betrachtungsweise des BGH auch bereits zuvor im Zusammenhang mit der Höchstpersönlichkeit auf S. $95 \mathrm{f}$.

465 BGH a.a.O (Fn. 464).

466 Martini/Kienle, JZ 2019, 235, 237 f.; zweifelnd auch Preuß, NJW 2018, 3146, 3147; a.A., dem BGH zustimmend, hingegen Härting/Dag, K\&R 2018, 638, 639.

467 Reimer in: HK-DS-GVO, Art. 6 Rn. 20; Frenzel in: Paal/Pauly (Hrsg.), Art. 6 DSGVO Rn. 14; ferner Albers/Veit in: BeckOK Datenschutzrecht, Art. 6 DS-GVO Rn. 32. 
den Vertragszweck ohne oder zumindest mit einem Weniger an Datenverarbeitung zu erreichen. ${ }^{468}$ Ein solch wertender Ansatz läuft letztlich aber auf eine Interessenabwägung hinaus, die aus systematischen Gründen dem dafür vorgesehenen Art. 6 Abs. 1 S. 1 lit.f) DS-GVO überlassen bleiben sollte. ${ }^{469} \mathrm{Im}$ Rahmen von lit.f) ist das Merkmal der Erforderlichkeit selbst eine von mehreren Tatbestandsvoraussetzungen. ${ }^{470}$ Für lit. b) nun in der Erforderlichkeit alleine - der Sache nach - einen Verweis auf den (gesamten) Tatbestand von lit.f) zu sehen, würde der Systematik der Erlaubnistatbestände nicht gerecht.

Unter Bezugnahme auf die bereits erwähnte Verwandtschaft zur Einwilligung ist nach einem engeren Ansatz daher richtigerweise entscheidend, ob die Datenverarbeitung für die betroffene Person im Vertrag so klar angelegt war, dass der Verantwortliche sich unter Verweis auf den Vertragsschluss auf die Willensbekundung der betroffenen Person stützen kann. ${ }^{471}$ Spiegelbildlich ist zu prüfen, ob sich die betroffene Person treuwidrig verhält, wenn sie die Datenverarbeitung (nachträglich) ablehnt. ${ }^{472}$

Durch den Abschluss eines Nutzungsvertrags mit dem Betreiber eines sozialen Netzwerks bekundet der Nutzer seine Billigung einer weitgehenden Datenverarbeitung. Es ist für den einzelnen Nutzer hinreichend deutlich erkennbar, dass die Inanspruchnahme der unterschiedlichen Kommunikations- und Informationsfunktionen nicht ohne umfangreiche Datenverarbeitungsvorgänge möglich ist. Auf diese Willensentschließung muss sich der Provider verlassen können. Indes gehört die Zugangsverschaffung gegenüber den Erben - beispielsweise in Form der Mitteilung oder Zurücksetzung des Passworts des Erblassers - gerade nicht zu der vernünftigen Erwartung des Kommunikationspartner des Verstorbenen. ${ }^{473}$ Konsequenterweise kann danach in dem Abschluss des Nutzungsvertrags auch keine die Verarbeitung legitimierende Willensbekundung verortet werden. Es ist mithin für die Erfüllung des Vertrages zwischen Provider und

468 Kübling/Klar/Sackmann, Datenschutzrecht, Rn. 371; Buchner/Petri in: Kühling/ Buchner (Hrsg.), Art. 6 DS-GVO Rn. 45; in diese Richtung auch Albers/Veit in: BeckOK Datenschutzrecht, Art. 6 DS-GVO Rn. 32.

469 Schantz in: NK-Datenschutzrecht, Art. 6 Abs. 1 DS-GVO Rn. 32.

470 Ausführlich zu Art. 6 Abs. 1 S. 1 lit. f) DS-GVO sogleich auf S. 130 ff. Zu den Voraussetzungen von lit. f) vergleiche die Nachweise in Fn. 476.

471 Schantz in: NK-Datenschutzrecht, Art. 6 Abs. 1 DS-GVO Rn. 32; ähnlich so auch Albrecht/Jotzo, Das neue Datenschutzrecht der EU, Teil 3 Rn. 43.

472 Ebd. Fn. 469.

473 Vgl. dazu bereits auf S. 97 ff. sowie die Nachweise zur Gegenansicht in Fn. 331. 
Kommunikationspartner nicht erforderlich i.S.v. lit.b), dass der Provider den Erben Zugang zum Nutzungskonto des Verstorbenen verschafft. ${ }^{474}$

\section{(3) Zwischenergebnis zu bb)}

Das Merkmal der Erforderlichkeit aus Art. 6 Abs. 1 S. 1 lit. b) Var. 1 DSGVO steht systematisch und teleologisch in Zusammenhang mit dem Erlaubnistatbestand der Einwilligung aus lit. a). Bei der Bestimmung des zur Vertragserfüllung Erforderlichen ist daher auf das vertragliche Pflichtenregime sowie auf die im Vertragsschluss ausgedrückte Willensbekundung der betroffenen Person zu rekurrieren. Auf dieser Grundlage und angesichts der Erwartungshaltung eines verständigen Nutzers kann der Vertrag zwischen den Kommunikationspartnern und dem Provider nicht zum Anknüpfungspunkt dafür gemacht werden, den Erben Zugang zu verschaffen zu einem vom Vertrag gänzlich unabhängigen Account (i.e. dem des Erblassers).

cc) Wahrung berechtigter Interessen, Art. 6 Abs. 1 S. 1 lit. f) DS-GVO

Art. 6 Abs. 1 S. 1 lit.f) DS-GVO ermöglicht die Datenverarbeitung auf Grundlage einer Interessenabwägung. Danach ist die Verarbeitung rechtmäßig, wenn sie „zur Wahrung der berechtigten Interessen des Verantwortlichen oder eines Dritten erforderlich [ist], sofern nicht die Interessen oder Grundrechte und Grundfreiheiten der betroffenen Person, die den Schutz personenbezogener Daten erfordern, überwiegen [...].“ Bei lit. f) handelt es sich um eine Auffangvorschrift, die in Ansehung des abschlieBenden Charakters von Art. 6 Abs. 1 DS-GVO dazu dient, den von lit. a) bis e) nicht erfassten Fällen praktisch Rechnung zu tragen. ${ }^{475}$ Der Erlaub-

474 Im Ergebnis wie hier, aber mit etwas anderer Begründung Martini/Kienle, JZ 2019, 235, 237 f.; offenlassend Preuß, NJW 2018, 3146, 3147; a.A. BGH NJW 2018, 3178 Rn. 71 ff.; Härting/Dag, K\&R 2018, 638, 639.

475 Kühling/Klar/Sackmann, Datenschutzrecht, Rn. 399; ausführlich Albrecht/Jotzo, Das neue Datenschutzrecht der EU, Teil 3 Rn. 51; Buchner/Petri in: Kühling/ Buchner (Hrsg.), Art. 6 DS-GVO Rn. 141; a.A., nicht Auffang- sondern Ausnahmetatbestand, Frenzel in: Paal/Pauly (Hrsg.), Art. 6 DS-GVO Rn. 26. Im Ergebnis ist die hohe tatsächliche Bedeutung der Vorschrift aber einhellige Meinung. 
nistatbestand hat eine dreiteilige Struktur: ${ }^{476}$ Berechtigte Interessen des Verantwortlichen bzw. Dritter (dazu (1)), die Erforderlichkeit der Datenverarbeitung (dazu (2)) sowie kein Überwiegen der berechtigten Interessen des Betroffenen (dazu (3)). Die Abwägung hat dabei prinzipiell anhand der konkreten Umstände eines jeweiligen Einzelfalls zu erfolgen. ${ }^{477}$ Ferner ist es nach dem Wortlaut von lit.f) eigens zu würdigen, wenn es sich bei der betroffenen Person um ein Kind handelt (dazu (4)). Schließlich kann eine auf lit.f) gestützte Datenverarbeitung ein Widerspruchsrecht der betroffenen Person gem. Art. 21 Abs. 1 DS-GVO auslösen (dazu (5)).

\section{(1) Berechtigte Interessen des Verantwortlichen bzw. Dritter}

Der Begriff des berechtigten Interesses ist weit auszulegen. ${ }^{478}$ Dafür sprechen nicht zuletzt die Erwägungsgründe 47 bis 49, in denen ein breites Spektrum legitimer Interessen exemplarisch aufgezählt wird. ${ }^{479}$ Berücksichtigungsfähig ist grundsätzlich jedes rechtliche, tatsächliche, wirtschaftliche oder ideelle Interesse. ${ }^{480}$ Dabei kann es sich bei den geltend gemachten Interessen ausweislich des insoweit eindeutigen Wortlauts von lit.f) um solche des Verantwortlichen selbst oder um solche von Dritten handeln. ${ }^{481}$

476 Vgl. dazu Frenzel in: Paal/Pauly (Hrsg.), Art. 6 DS-GVO Rn. 27 ff.; allgemein zu den Voraussetzungen von lit.f) siehe Buchner/Petri in: Kühling/Buchner (Hrsg.), Art. 6 DS-GVO Rn. 141 ff.; Reimer in: HK-DS-GVO, Art. 6 Rn. 52 ff.; mit Vorschlag zur praktischen Handhabung der Abwägung Herfurth, ZD 2018, 514, $514 \mathrm{ff}$.

477 Albers/Veit in: BeckOK Datenschutzrecht, Art. 6 DS-GVO Rn. 49, 51; BGH NJW 2018, 3178 Rn. 75 unter Verweis auf EuGH NJW 2016, 3579 Rn. 62.

478 Frenzel in: Paal/Pauly (Hrsg.), Art. 6 DS-GVO Rn. 28; ausführlich Taeger in: Taeger/Gabel (Hrsg.), Art. 6 DS-GVO Rn. 103 ff.

479 Frenzel in: Paal/Pauly (Hrsg.), Art. 6 DS-GVO Rn. 28; BGH NJW 2018, 3178 Rn. 76 weist hin auf die in den Erwägungsgründen genannten Beispiele wie etwa die Verhinderung von Betrug, die Direktwerbung, konzerninterne Übermittlungen oder die Verbesserung der Netz- und Informationssicherheit.

480 BGH NJW 2018, 3178 Rn. 76; Buchner/Petri in: Kühling/Buchner (Hrsg.), Art. 6 DS-GVO Rn. 146a; Reimer in: HK-DS-GVO, Art. 6 Rn. 54; Taeger in: Taeger/ Gabel (Hrsg.), Art. 6 DS-GVO Rn. 107.

481 Kühling/Klar/Sackmann, Datenschutzrecht, Rn.401; für einen Vergleich der Rechtslage vor und unter Geltung der DS-GVO s. Taeger in: Taeger/Gabel (Hrsg.), Art. 6 DS-GVO Rn. 104 und Schantz/Wolff, Das neue Datenschutzrecht, Rn. 645. 
Den Schwerpunkt der bisherigen Debatte über die Zugangsgewährung gegenüber den Erben haben die Interessen der Erben gebildet. ${ }^{482}$ Hier sind vor allem das durch Art. 14 Abs. 1 S. 1 GG garantierte Erbrecht sowie der in $\$ 1922$ Abs. 1 BGB ausgeprägte Grundsatz der Universalsukzession zu nennen. Diese Positionen drohen ausgehöhlt zu werden, wenn der im Wege des Erbgangs übergegangene vertragliche Anspruch auf Zugang zum Erblasseraccount durch entgegenstehendes Datenschutzrecht (grundsätzlich) vereitelt würde. ${ }^{483}$ Außerdem trifft die Erben das Recht und die Pflicht zur Fortführung des Geschäftsverlaufs beziehungsweise zur Abwicklung noch ausstehender Geschäfte des Erblassers. ${ }^{484}$ Nach $\$ 130$ Abs. 2 BGB bleibt eine Willenserklärung auch dann wirksam, wenn der Erklärende nach der Abgabe stirbt. Folgerichtig steht der Tod des Antragenden gem. $\$ 153$ BGB regelmäßig auch dem wirksamen Zustandekommen eines Vertrages nicht im Wege. So sind die Erben unmittelbar nach dem Erbfall darauf angewiesen, zügig und umfassend Informationen zur Zusammensetzung und zum Wert der Erbschaft zu erhalten. ${ }^{485}$ Dazu gehören insbesondere die Nachlassverbindlichkeiten, für welche die Erben gem. $\$ 1967$ BGB haften. ${ }^{486}$ Die Frist zur Ausschlagung der Erbschaft beträgt im Regelfall sechs Wochen ab Kenntnis des Erben von der Erbschaft und seiner Erbenstellung, vgl. $\$ 1944$ BGB. ${ }^{487}$ Die Nachlassermittlung würde ohne Zugang zu den Nutzerkonten des Erblassers erschwert. Denn mitunter lassen sich Hinweise auf die Aktiva und Passiva des Nachlasses (nur) aus dem EMail-Verkehr des Erblassers oder sonstigen digitalen Speichern (bspw. di-

482 Mit unterschiedlichen Ergebnissen vgl. BGH NJW 2018, 3178 Rn. 78 ff. einerseits sowie Martini/Kienle, JZ 2019, 235, 238 andererseits.

483 Vgl. BGH NJW 2018, 3178 Rn. 78 nach dem es „bereits für sich genommen ein gewichtiges berechtigtes Interesse eines Vertragspartners dar[stellt], die Hauptleistungsansprüche aus [einem] Vertragsverhältnis auch geltend machen zu können."

484 Kutscher, Der digitale Nachlass, S. 18 f.; zur Pflicht das Impressum einer Webseite des Erblassers nach dem Erbfall zu aktualisieren Hoeren, NJW 2005, 2113, $2116 \mathrm{f}$. , der dafür eine Frist von 6 Wochen annimmt; Thiesen, Daten in der Erbmasse, S. 8, betont das Abmahnrisiko, wenn diese Pflicht nicht fristgerecht erfüllt wird; Bock, AcP (217) 2017, 370, 397 weist auf sonstige Fristen - insbesondere Kündigungsfristen - hin, die von den Erben zu beachten seien; s. auch Funk, Das Erbe im Netz, S. 6.

485 Budzikiewicz, AcP (218) 2018, 558, 562; Klas/Möbrke-Sobolewski, NJW 2015, 3473, 3474; Bock, AcP (217) 2017, 370, 396.

486 Thiesen, Daten in der Erbmasse, S. 8; Küpper in: MüKo BGB, $\$ 1967$ Rn. 2 ff.; Hoeren in: HK-BGB, $\mathbb{S} 1967$ Rn. $3 \mathrm{ff}$.

487 Vgl. dazu Leipold in: MüKo BGB, $\mathbb{1} 1944$ Rn. 3 ff.; Siegmann/Höger in: BeckOK BGB, $\mathbb{1} 1944$ Rn. 2 ff. 
gitalisierten Vertragsdokumenten in cloudbasierten Speichern des Erblassers) entnehmen. ${ }^{488}$ Bei der Nachlassermittlung kommt dem E-Mail-Konto des Erblassers eine Schlüsselfunktion zu, da über das E-Mail Postfach regelmäßig sowohl Kenntnis von als auch Zugang zu den sonstigen Nutzerkonten des Erblassers (bei sozialen Netzwerken, Streamingdiensten, Partnerbörsen, Online-Spielen etc.) erlangt werden kann. ${ }^{489}$ Das dringende Interesse der Erben an der Nachlassermittlung wird durch die Regelung der $\$ \mathbb{S} 1993 \mathrm{ff}$. BGB weiter unterstrichen. ${ }^{490}$ Danach haben die Erben auf Antrag eines Nachlassgläubigers innerhalb einer gerichtlichen Frist ein Inventar über den Nachlass zu erstellen. Nach erfolglosem Ablauf der Frist droht den Erben eine unbeschränkte Nachlasshaftung. ${ }^{491}$ Schließlich kommen potenziell Interessen der Erben hinzu, die vor allem emotionaler Natur sein werden wie etwa die Trauerbewältigung oder Erinnerungspflege. 492

Daneben können durchaus auch eigene berechtigte Interessen des Providers an der Zugangsgewährung gegenüber den Erben bestehen. Erfolgreiche und reichweitestarke Accounts, zum Beispiel eines Influencers, steigern die Attraktivität des Netzwerks für mögliche neue Nutzer. Es ist somit zumindest nicht ausgeschlossen, dass die Zugangsgewährung gegenüber den Erben es ermöglicht, solche Accounts aufrechtzuerhalten und damit die wirtschaftlichen Interessen des Providers zu fördern.

\section{(2) Erforderlichkeit der Datenverarbeitung}

Die Datenverarbeitung muss nach lit. f) zur Wahrung der berechtigten Interessen erforderlich sein. Das Merkmal der Erforderlichkeit steht den sehr

488 Auer-Reinsdorff in: Auer-Reinsdorff/Conrad (Hrsg.), Handbuch IT- und Datenschutzrecht, $\mathbb{S} 21 \mathrm{Rn} .84$; anschaulich insoweit auch Sorge, MMR 2018, 372, 374.

489 Abhängig von der technischen Gestaltung des Zugangs lassen sich Passwörter in vielen Fällen allein mittels des E-Mail-Kontos zurücksetzen. Sofern ein Provider für den Zugang oder die Zurücksetzung der Zugangsdaten eine Zwei-FaktorAuthentifizierung eingerichtet hat, sind die Rechtsnachfolger auf Auskunftsansprüche gegen den Provider angewiesen.

490 So auch bereits Thiesen, Daten in der Erbmasse, S. 8.

491 Vgl. dazu Küpper in: MüKo BGB, $\mathbb{1} 1994$ Rn. 11 ff.; Hoeren in: HK-BGB, $\mathbb{1} 1994$ Rn. 1 ff.; Lohmann in: BeckOK BGB, $\$ 1994$ Rn. $11 \mathrm{ff}$.

492 Beispiele für einzelfallspezifische Interessen finden sich bei BGH NJW 2018, 3178 Rn. 80 f. (u.a. Suizidabsichten der Erblasserin). 
weiten berechtigten Interessen als ein normatives Korrektiv gegenüber. ${ }^{493}$ Entscheidend ist, ob eine bestimmte Form der Datenverarbeitung zur Erreichung eines konkreten Zwecks das relativ mildeste Mittel ist. ${ }^{494}$ In Ansehung des Grundsatzes der Datenminimierung aus Art. 5 Abs. 1 lit. c) DSGVO ist zur möglichst weitgehenden Schonung der betroffenen Person derjenige Weg zur Wahrung der berechtigten Interessen zu wählen, welcher unter mehreren gleichermaßen geeigneten Mitteln die niedrigste Eingriffsintensität aufweist. ${ }^{495}$

In seiner Facebook-Entscheidung stellt der BGH ohne nähere Prüfung fest, dass neben der (vollständigen) Zugangsgewährung zum Account des Erblassers keine geeigneteren und milderen Mittel bestünden, um die berechtigten Interessen der Erben zu erfüllen. ${ }^{496}$ Dabei erscheint es zumindest nicht von vorneherein ausgeschlossen, dass die Erbeninteressen bereits durch einen eingeschränkten Zugang zu den Accountinhalten in Form von anonymisierten oder pseudonymisierten Daten gewahrt werden können. ${ }^{497}$ Denkbar wäre hier auch eine Zugangsgewährung in zwei Schritten: ${ }^{498}$ In einem ersten Schritt könnten den Erben zunächst nur die Nachrichteninhalte ohne die Identität der Absender eröffnet werden; erst im Anschluss und bei Fortbestehen der Erbeninteressen hinsichtlich bestimm-

493 Martini/Kienle, JZ 2019, 235, 238; Albers/Veit in: BeckOK Datenschutzrecht, Art. 6 DS-GVO Rn. 50. Insofern handelt es sich funktional nicht um denselben Erforderlichkeitsbegriff wie im Rahmen von Art. 6 Abs. 1 S. 1 lit. b) Var. 1 DSGVO, zu diesem siehe bereits zuvor auf S. $128 \mathrm{f}$.

494 Schantz/Wolff, Das neue Datenschutzrecht, Rn. 431 mit Verweis auf EuGH NVwZ 2009, 379 Rn. 65 zu Art. 7 lit. e) DSRL; Martini/Kienle, JZ 2019, 235, 238; ähnlich Schantz in: NK-Datenschutzrecht, Art. 6 Abs. 1 DS-GVO Rn. 100; ferner Heberlein in: Ehmann/Selmayr (Hrsg.), Art. 6 DS-GVO Rn. 28: „das absolut Notwendige."

495 Martini/Kienle, JZ 2019, 235, 238; Schantz in: NK-Datenschutzrecht, Art. 6 Abs. 1 DS-GVO Rn. 100; einschränkend Kramer in: Auernhammer, Art. 6 DSGVO Rn. 79 f.; Plath in: Plath (Hrsg.), Art. 6 DS-GVO Rn. 56 f., 18; keine Filterwirkung vom Merkmal der Erforderlichkeit erwartet Reimer in: HK-DS-GVO, Art. 6 Rn. 54; nicht ganz so weitgehend Taeger in: Taeger/Gabel (Hrsg.), Art. 6 DS-GVO Rn. 112 nach dem das Merkmal aber zumindest „dem unternehmerischen Streben nach Effizienz und wirtschaftlicher Unternehmensführung nicht entgegengehalten werden" kann.

496 BGH NJW 2018, 3178 Rn. 82.

497 So auch Martini/Kienle, JZ 2019, 235, 238 f., die u.a. exemplarisch auf Art. 32 Abs. 1 lit. a) DS-GVO verweisen; allgemein zur Anonymisierung im Rahmen der Erforderlichkeitsprüfung Schantz/Wolff, Das neue Datenschutzrecht, Rn. 431 sowie Schantz in: NK-Datenschutzrecht, Art. 6 Abs. 1 DS-GVO Rn. 100.

498 Andeutungsweise in diese Richtung Martini/Kienle, a.a.O. (Fn. 497). 
ter Nachrichtenverläufe könnte sodann in einem zweiten Schritt die Identität von Kommunikationspartnern preisgegeben werden.

Zwar ist dem BGH zuzugeben, dass es für das Verständnis der Korrespondenz in der Regel von Belang sein dürfte, wer eine bestimmte Nachricht an den Erblasser verschickt hat. Es sind aber zumindest auch Konstellationen denkbar, in denen der unbeschränkte Zugriff der Erben auf den Erblasseraccount nicht erforderlich ist. Zum einen seien beispielhaft Fälle genannt, in denen das Interesse an den gespeicherten Inhalten aus der Vergangenheit ohnehin nur nachrangig ist gegenüber dem Interesse an der Nutzungsmöglichkeit des Accounts für die Zukunft. ${ }^{499}$ Zum anderen kommen Sachverhalte in Betracht, in denen das Informationsinteresse der Erben bereits durch den Kommunikationsinhalt unabhängig von der Identität der Kommunizierenden befriedigt werden kann. Freilich mag es sich dabei zwar nicht um den Regelfall handeln, gleichwohl ist diese Möglichkeit aber ernsthaft und im Einzelfall zu prüfen. ${ }^{500}$ Denn erforderlich ist die Datenverarbeitung in Form der (vollständigen) Zugangsgewährung nur, wenn (und soweit) sie sich nach der Prüfung denkbarer Alternativen als das relativ mildeste Mittel herausstellt.

\section{(3) Kein Überwiegen der Interessen der betroffenen Person}

Den berechtigten Interessen des Verantwortlichen bzw. Dritter dürfen nach Art. 6 Abs. 1 S. 1 lit. f) DS-GVO keine überwiegenden Interessen oder Grundrechte und Grundfreiheiten der betroffenen Person gegenüberstehen. Da lit. f) die Verarbeitung personenbezogener Daten gerade ohne Einwilligung der Betroffenen gestatten kann, sind regelmäßig berührt Art. 7 GRCh, das Grundrecht auf Achtung des Privat- und Familienlebens sowie der Kommunikation, und Art. 8 GRCh, das Grundrecht auf Schutz der personenbezogenen Daten. ${ }^{501}$ Darüber hinaus ist der Begriff des Interesses des Betroffenen ebenso weit auszulegen, wie die Interessen des Verarbei-

499 Siehe dazu bereits (bei) Fn. 348.

500 Zur einzelfallspezifischen Anwendung von Art. 6 Abs. 1 S. 1 lit. f) DS-GVO vgl. bereits die Nachweise in Fn. 477.

501 Schantz in: NK-Datenschutzrecht, Art. 6 Abs. 1 DS-GVO Rn. 101; Kühling/Klar/ Sackmann, Datenschutzrecht, Rn. 403; Heberlein in: Ehmann/Selmayr (Hrsg.), Art. 6 DS-GVO Rn. 28; das gilt unabhängig davon, dass der (private) Verarbeitende gem. Art. 51 Abs. 1 S. 1 GRCh selbst nicht unmittelbar an die GRCh gebunden ist, vgl. Frenzel in: Paal/Pauly (Hrsg.), Art. 6 DS-GVO Rn. 30. 
tenden bzw. der Dritten, sodass einzelfallspezifisch weitere Betroffeneninteressen neben Art. 7, 8 GRCh treten können.502

Ein zentraler Aspekt der Abwägung zwischen den widerstreitenden Interessen ist nach Erwägungsgrund 47 S. 1 und 3 die ,vernünftige Erwartung" der betroffenen Person hinsichtlich der konkret infrage stehenden Datenverarbeitung. ${ }^{503}$ Dabei ist ein objektivierter Maßstab anzulegen. ${ }^{504}$ $\mathrm{Zu}$ fragen ist demnach, ob die Datenverarbeitung für einen objektiven Dritten in der Situation des Betroffenen vorhersehbar wäre. Insofern kann wiederum auf die Ausführungen zur Erwartungshaltung eines durchschnittlichen Nutzers sozialer Medien im Rahmen der Höchstpersönlichkeit verwiesen werden: Während der BGH in seinem Facebook-Urteil unter Zustimmung von Teilen der Literatur davon ausgeht, dass der Übergang eines Nutzerkontos auf die Erben samt Zugriffsrecht zur vernünftigen Erwartung der Kommunikationspartner gehöre, ${ }^{505}$ ist eine dahingehende Erwartungshaltung nach hiesiger Auffassung nicht lebensnah und sollte daher nicht unterstellt werden. ${ }^{506}$

Neben den vernünftigen Erwartungen der betroffenen Person argumentiert der BGH weiterhin damit, dass dem Eingriff in die Interessen der Kommunikationspartner hier nur ein geringes Gewicht zukomme. ${ }^{507}$ Die Verwirklichung des Erbrechts in Form der Zugangsgewährung stellt nach Ansicht des BGH einen eng umgrenzten Zweck dar, welchem das Gericht Beispiele mit einem (vermeintlich) weitergehendem Zweck gegenüberstellt, die hier nicht einschlägig sind, wie etwa Werbung oder Profilbil-

502 Heberlein in: Ehmann/Selmayr (Hrsg.), Art. 6 DS-GVO Rn. 28; Schantz in: NKDatenschutzrecht, Art. 6 Abs. 1 DS-GVO Rn. 101 nennt beispielhaft wirtschaftliche Interessen des Betroffenen, sein Ansehen in der Öffentlichkeit oder diskriminierende (Folge-)Effekte der etwaigen Datenverarbeitung.

503 Heberlein in: Ehmann/Selmayr (Hrsg.), Art. 6 DS-GVO Rn. 28; ferner Kramer in: Auernhammer, Art. 6 DS-GVO Rn. 83 ff. mit einer produktiven Unterscheidung zwischen vernünftiger und tatsächlicher Vorhersehbarkeit der Verarbeitung für Betroffene.

504 Frenzel in: Paal/Pauly (Hrsg.), Art. 6 DS-GVO Rn.30; Tavanti, RDV 2016, 295, 299; Kramer in: Auernhammer, Art. 6 DS-GVO Rn. 84.

505 Vgl. dazu bereits die Nachweise in Fn. 331. Das Gericht bekräftigt diese Ansicht auch und gerade im Rahmen seiner datenschutzrechtlichen Prüfung, vgl. BGH NJW 2018, 3178 Rn. 90 f. diesbezüglich zustimmend Härting/Dag, K\&R 2018, 638, 639 sowie Apel, ZD 2018, 486, 487.

506 Siehe dazu zuvor auf S. $97 \mathrm{ff}$.

507 BGH NJW 2018, 3178 Rn. 92; a.A. Martini/Kienle, JZ 2019, 235, 239 und dort in Fn. 58, welche vor der Offenlegung gegenüber „einer rechtlich nicht beschränkten Zahl an Erben ( $\$ 2032$ BGB)“ warnen. 
dung. ${ }^{508}$ Dieser Vergleich hält einer Überprüfung nur in Bezug auf die Profilbildung stand. Hinsichtlich der Werbezwecke ist dem Gericht insofern zu widersprechen, als dass die betroffene Person sich der Verarbeitung ihrer personenbezogenen Daten zu Werbezwecken bei der Nutzung des Sozialen Netzwerks bewusst und dauerhaft aussetzt. ${ }^{509}$ In vielen Fällen wird sie in die Verarbeitung zu Werbezwecken bei Abschluss des Nutzungsvertrags sogar eingewilligt haben. Der Zugang der Erben zur Korrespondenz zwischen dem Erblasser und der betroffenen Person stellt hingegen eine neue, im bisherigen Nutzungsverhältnis ungewöhnlichen Verarbeitungszweck dar, der von der Einwilligung des Betroffenen regelmäßig gerade nicht umfasst sein wird. ${ }^{510}$ Daher ist es kaum überzeugend, wenn der BGH eine Hierarchie der Eingriffsintensität insinuiert, der zufolge es dem Betroffenen eher zumutbar sein soll, dass die auf ihn bezogenen Daten den Erben (erstmals) zugänglich werden, als dass sich die ohnehin schon laufende Verarbeitung des Providers zu Werbezwecken fortsetzt.

\section{(4) Kinder im Rahmen von Art. 6 Abs. 1 S. 1 lit. f) DS-GVO}

Art. 6 Abs. 1 S. 1 lit. f) DS-GVO bestimmt a.E., dass eine Datenverarbeitung auf Grundlage berechtigter Interessen nur rechtmäßig ist, sofern nicht die Interessen des Betroffenen überwiegen, ,insbesondere dann, wenn es sich bei der betroffenen Person um ein Kind handelt. " ${ }^{111}$ Es ist umstritten, welche Konsequenzen aus diesem Passus folgen. Weitgehend Einigkeit besteht noch darüber, dass unter einem Kind im Grundsatz ein Minderjähriger bis zur Vollendung des 18 . Lebensjahres zu verstehen ist. ${ }^{512}$ Eine strikt grammatikalische Auslegung von lit.f) a.E. ließe den Schluss zu, dass in dem

508 BGH a.a.O. (Fn. 507).

509 Vgl. dazu bereits zuvor auf S. $37 \mathrm{ff}$.

510 Anderenfalls käme es auf die Abwägung nach Art. 6 Abs. 1 S. 1 lit. f) DS-GVO schon nicht an. Zur Einwilligung nach lit. a) siehe S. $124 \mathrm{f}$.

511 Ausführlich zum Datenschutz von Kindern in der DS-GVO vgl. Roßnagel, ZD 2020, 88, passim sowie (rechtspolitisch) das Gutachten der Datenethikkommission vom 23.10.2019, S. 114 f., abrufbar a.a.O. (Fn. 30); im Kontext des digitalen Nachlasses (aber bezogen auf den Erblasser statt auf die Kommunikationspartner) z.B. Knoop, NZFam 2016, 966, $967 \mathrm{f}$.

512 Schantz in: NK-Datenschutzrecht, Art. 6 Abs. 1 DS-GVO Rn. 112 und Heberlein in: Ehmann/Selmayr (Hrsg.), Art. 6 DS-GVO Rn. 31 verweisen auf Art. 1 UNKinderrechtskonvention; Buchner/Petri in: Kühling/Buchner (Hrsg.), Art. 6 DSGVO Rn. 155; a.A. Martini/Kienle, JZ 2019, 235, 239 die anstelle einer Altersgrenze auf die Einsichtsfähigkeit des Kindes abstellen wollen. 
Fall eines Kindes als Betroffenem stets ein Überwiegen der Betroffeneninteressen zu sehen sei. Ein solche Lesart hätte zur Folge, dass die Verarbeitung von Daten, die sich auf Kinder beziehen, grundsätzlich nicht auf lit. f) gestützt werden könnte. Dies nehmen einige Stimmen in der Literatur unter Verweis auf Art. 8 Abs. 1 S. 1 DS-GVO zumindest für unter 16-jährige Betroffene ${ }^{513}$ bzw. mit Verweis auf Art. 8 Abs. 1 UAbs. 2 DS-GVO jedenfalls für unter 13-jährige Betroffene ${ }^{514}$ an. Andere Stimmen wollen den Zusatz in lit. f) a.E. statt an einer Altersgrenze eher thematisch orientieren und ein Überwiegen der Betroffeneninteressen dann annehmen, wenn einer der in Erwägungsgrund 38 genannten Risikofälle vorliegt. ${ }^{515}$ Dazu gehört die Verarbeitung von auf Kinder bezogenen Daten für Werbezwecke, für die Erstellung von Persönlichkeitsprofilen oder für direkt auf Kinder ausgerichtete Dienste. Wiederum andere Teile der Literatur lehnen jedwede starre Lesart von lit.f) a.E. ab und fordern lediglich ein erhöhtes Gewicht der Kindesinteressen in einer nach wie vor durchzuführenden Abwägung im Einzelfall. ${ }^{516}$

Gemeinsam ist allen Ansichten, dass bei der Verarbeitung auf Grundlage von lit.f) zwischen den Daten Erwachsener und denen von Kindern zu unterscheiden ist. In Bezug auf die Zugangsverschaffung zum Erblasseraccount kommt diese Unterscheidung zum Tragen, wenn sich unter den Kommunikationspartnern des Erblassers (auch) Kinder befunden haben (könnten). ${ }^{517}$ Unabhängig vom Alter des Betroffenen sind dessen „vernünftige Erwartungen" hinsichtlich der konkret infrage stehenden Datenverarbeitung mittels eines objektivierten Maßstabs zu bestimmen. ${ }^{518}$ Bei dieser Maßstabsbildung sind die begrenzte Einsichtsfähigkeit eines Kindes sowie seine beschränkte Befähigung zur Risikofolgenabschätzung entschei-

513 Buchner/Petri in: Kühling/Buchner (Hrsg.), Art. 6 DS-GVO Rn. 155.

514 Albers/Veit in: BeckOK Datenschutzrecht, Art. 6 DS-GVO Rn. 51; so wohl auch Schantz in: NK-Datenschutzrecht, Art. 6 Abs. 1 DS-GVO Rn. 112.

515 Schantz in: NK-Datenschutzrecht, Art. 6 Abs. 1 DS-GVO Rn. 112.

516 So Reimer in: HK-DS-GVO, Art. 6 Rn. 64; Martini/Kienle, JZ 2019, 235, 239; wohl auch Heberlein in: Ehmann/Selmayr (Hrsg.), Art. 6 DS-GVO Rn. 31; den Zusatz in lit. f) a.E. als deklaratorisch bezeichnend Schulz in: Gola (Hrsg.), Art. 6 DS-GVO Rn. 59.

517 Davon ist insbesondere dann auszugehen, wenn der Erblasser selbst minderjährig war, vgl. BGH NJW 2018, 3178 Rn. 85; hingegen auf den Schutz des minderjährigen Erblassers (!) abstellend Hoeren, MMR 2018, 749, 749.

518 Siehe hierzu bereits S. 135 f. sowie die Nachweise in Fn. 503 und 504. 
dend zu berücksichtigen. ${ }^{519}$ Nach hier vertretener Ansicht gehört der Zugang der Erben zur Korrespondenz zwischen Erblasser und Kommunikationspartnern bereits nicht zur vernünftigen Erwartung erwachsener Nutzer. Das gilt sodann erst recht für Minderjährige, denen grundsätzlich nicht unterstellt werden kann, bei der Nutzung sozialer Medien den eigenen Tod oder den ihres Kommunikationspartners als Möglichkeit auch nur in Betracht zu ziehen. ${ }^{520}$

\section{(5) Widerspruchsrecht des Betroffenen, Art. 21 Abs. 1 S. 1 DS-GVO}

Der betroffenen Person steht gem. Art. 21 Abs. 1 S. 1 DS-GVO ein Widerspruchsrecht gegen eine auf Art. 6 Abs. 1 S. 1 lit.f) DS-GVO gestützte Verarbeitung zu, sofern dafür Gründe vorliegen, die sich aus der „besonderen Situation" des Betroffenen ergeben. ${ }^{521}$ Ist das der Fall, darf die Verarbeitung nicht bereits bei einem „,berechtigten Interesse“ im Sinne von Art. 6 Abs. 1 S. 1 lit.f) DS-GVO, sondern nur bei Vorliegen „zwingender schutzwürdiger Gründe“ oder zur „Geltendmachung, Ausübung oder Verteidigung von Rechtsansprüchen" erfolgen, vgl. Art. 21 Abs. 1 S. 2 DS-GVO. Ausweislich des Wortlauts der Norm geht ein „non liquet“ dabei zulasten des Verantwortlichen. ${ }^{522}$ Die betroffene Person muss durch den Verant-

519 Ausdrücklich in diesem Sinne Erwägungsgrund 38 S. 1: „Kinder verdienen bei ihren personenbezogenen Daten besonderen Schutz, da Kinder sich der betreffenden Risiken, Folgen und Garantien und ihrer Rechte bei der Verarbeitung personenbezogener Daten möglicherweise weniger bewusst sind." Siehe aber BGH NJW 2018, 3178 Rn. 90; Härting/Dag, K\&R 2018, 638, 639; zurecht kritisch zu dieser Rspr. Martini/Kienle, JZ 2019, 235, 239; ausführlich zur Nutzung sozialer Netzwerke durch Kinder und Jugendliche nach BDSG a.F., vgl. Jandt/ Roßnagel, MMR 2011, 637, $637 \mathrm{ff}$.

520 Alexander, notar 2018, 367, 367 fordert „die jüngeren und besonders internetaffinen Generationen“, die Fragen des digitalen Nachlasses „häufig noch als unangenehm verdrängen“, zur Auseinandersetzung mit diesem Thema auf.

521 Ausführlich hierzu im Kontext des digitalen Nachlasses Martini/Kienle, JZ 2019, 235, 240; allgemein zum Widerspruchsrecht Forgó in: BeckOK Datenschutzrecht, Art. 21 DS-GVO Rn. 1 ff.; Martini in: Paal/Pauly (Hrsg.), Art. 21 DS-GVO Rn. $1 \mathrm{ff}$.

522 Vgl. Forgó in: BeckOK Datenschutzrecht, Art. 21 DS-GVO Rn. 10; Martini in: Paal/Pauly (Hrsg.), Art. 21 DS-GVO Rn. 36; Martini/Kienle, JZ 2019, 235, 240; in Art. 21 Abs. 1 DS-GVO trotzdem nur eine „Härtefallregelung für Ausnahmesituationen“ zulasten des Betroffenen sieht Veil, NJW 2018, 3337, 3341; in diese Richtung auch Uecker, ZD 2019, 248, 249. 
wortlichen auf ihr Widerspruchsrecht hingewiesen werden, vgl. Art. 21 Abs. 4 DS-GVO. 523

Für den digitalen Nachlass lässt sich das Widerspruchsrecht als Möglichkeit zur Differenzierung zwischen unterschiedlichen Inhalten des Erblasseraccounts fruchtbar machen. Sofern die Abwägung nach Art. 6 Abs. 1 S. 1 lit.f) DS-GVO zumindest grundsätzlich den Zugang der Erben zum Account des Erblassers ermöglicht, muss der Provider die Kommunikationspartner des Erblassers gem. Art. 21 Abs. 4 DS-GVO über ihr Widerspruchsrecht gegen die Zugangsverschaffung gegenüber den Erben informieren. ${ }^{524}$ Solchen Kommunikationspartnern, die besonders intime Informationen bzw. Bilder mit dem Erblasser geteilt haben, ist dann die Möglichkeit des Widerspruchs eröffnet. Beruflichen Kontaktpersonen hingegen steht mangels „besonderer Situation“ entweder schon kein Widerspruchsrecht zu oder sie machen von ihrem Recht bewusst keinen Gebrauch, weil die Fortführung bzw. Abwicklung der Geschäfte des Erblassers durch die Erben sogar im Interesse geschäftlicher Kommunikationspartner stehen kann.

Die etwaige Ausübung des Widerspruchsrechts zeitigt sodann eine (nach Art. 6 Abs. 1 S. 1 lit.f) DS-GVO zweite) Abwägung zwischen den „zwingenden schutzwürdigen Gründen für die Verarbeitung“ und den „Interessen, Rechten und Freiheiten der betroffenen Person".525 Dadurch kann das im Rahmen von Art. 6 Abs. 1 S. 1 lit. f) DS-GVO abstrakt für den gesamten Account ermittelte Ergebnis (i.e. die umfassende Zugangsgewährung) durch die konkrete Abwägung nach Art. 21 Abs. 1 DS-GVO zugunsten einzelner besonders schutzwürdiger Kommunikationspartner des Erblassers korrigiert werden. Im Ergebnis steht den Erben dann ein weitgehender, aber um einzelne Nachrichtenverläufe gekürzter Zugang zu den Accountinhalten zu. Die Pflicht zur technischen Umsetzung dieser (erforderlichenfalls im Gerichtsprozess festgelegten) Differenzierung trifft zweckmäßigerweise den Betreiber des Sozialen Netzwerks als Verantwortlichen.

523 Herbst in: Kühling/Buchner (Hrsg.), Art. 21 DS-GVO Rn. 34 ff.; Martini in: Paal/ Pauly (Hrsg.), Art. 21 DS-GVO Rn. 64 ff.; Forgó in: BeckOK Datenschutzrecht, Art. 21 DS-GVO Rn. 24 ff.

524 Zum vorgeschriebenen Zeitpunkt des Hinweises gem. Art. 21 Abs. 4 DS-GVO vgl. Martini in: Paal/Pauly (Hrsg.), Art. 21 DS-GVO Rn. 66 ff. sowie Herbst in: Kühling/Buchner (Hrsg.), Art. 21 DS-GVO Rn. 37 f.

525 Martini/Kienle, JZ 2019, 235, 240. 
(6) Zwischenergebnis zu cc)

Um die Zugangsgewährung gegenüber den Erben auf Art. 6 Abs. 1 S. 1 lit.f) DS-GVO stützen zu können, muss eine Abwägung der im konkreten Einzelfall berührten Interessen durchgeführt werden. Dabei müssen namentlich das Verwendungsinteresse der Dritten (regelmäßig vor allem der Erben) mit dem Privatheitsinteresse der betroffenen Person (den Kommunikationspartnern des Erblassers) sorgfältig abgewogen werden. Unter dem Gesichtspunkt der Erforderlichkeit sind denkbare Alternativen zur Zugangsgewährung zu erwägen, mit denen die Erbeninteressen gewahrt werden können bei gleichzeitig größerer Schonung der Betroffeneninteressen. Dazu kann insbesondere gehören, nur einen eingeschränkten Zugang zum Erblasseraccount etwa unter Anonymisierung oder Pseudonymisierung der gespeicherten Inhalte zu gewähren, wenn und soweit das Informationsinteresse der Erben dadurch bereits (weitgehend) befriedigt werden kann. Wenn auch der Abwägung gem. lit.f) im Facebook-Urteil des $\mathrm{BGH}^{526}$ eine gewisse Vorbildwirkung zukommen mag, so befreit das Urteil nicht von der jeweiligen Prüfung im Einzelfall, in dem durchaus eine anders gelagerte Interessenkollision $\mathrm{zu}$ anderen Ergebnissen führen kann. ${ }^{527}$ Zwingend notwendig ist es, die besondere Schutzwürdigkeit der Interessen von Kindern als datenschutzrechtlich Betroffenen angemessen zu berücksichtigen und ihnen in der Abwägung ein besonderes Gewicht beizumessen. Wenn und soweit die Abwägung nach Art. 6 Abs. 1 S. 1 lit. f) DS-GVO zugunsten der Verarbeitung ausfällt, begründet dies ein Widerspruchsrecht der betroffenen Person nach Art. 21 Abs. 1 S. DS-GVO, worauf die Kommunikationspartner als Betroffene gem. Art. 21 Abs. 4 DSGVO hingewiesen werden müssen. Der Widerspruch besonders schutzwürdiger Kommunikationspartner bewirkt, dass ihre Korrespondenz mit dem Erblasser von dem Zugangsrecht der Erben ausgenommen sein kann.

526 BGH NJW 2018, 3178 Rn. $74 \mathrm{ff}$.

527 An „verschiedenen Stellen der Urteilsbegründung einzelfallorientierte Abwägungen vorherrschen" sieht auch Hoeren, MMR 2018, 749, 750; noch weiter gehen Martini/Kienle, JZ 2019, 235, 238, die das Urteil als "Sonderfall“ bezeichnen; differenzierend Preuß, NJW 2018, 3146, 3147. 
c) Verarbeitung „sensibler“ Daten im Sinne von Art. 9 DS-GVO

Die DS-GVO unterscheidet zwischen unterschiedlichen Kategorien von personenbezogenen Daten. Während sich die Zulässigkeit der Verarbeitung „einfacher" 528 personenbezogener Daten nach Art. 6 DS-GVO richtet, ${ }^{529}$ stellt Art. 9 DS-GVO ein Sonderregime für die „Verarbeitung besonderer Kategorien personenbezogener Daten" auf. Im Verhältnis zu Art. 6 Abs. 1 DS-GVO geht Art. 9 DS-GVO als lex specialis vor, ${ }^{530}$ sodass eine Verarbeitung nicht auf Art. 6 Abs. 1 DS-GVO gestützt werden kann, wenn und soweit Art. 9 DS-GVO dem entgegensteht. ${ }^{531}$ Während Art. 9 Abs. 1 DS-GVO den Anwendungsbereich der Norm bestimmt und ein Verarbeitungsverbot aufstellt (dazu aa), sieht Art.9 Abs. 2 DS-GVO abschließende Ausnahmetatbestände vor (dazu bb).

\section{aa) Das Verarbeitungsverbot aus Art. 9 Abs. 1 DS-GVO}

Die von Art. 9 DS-GVO erfassten Daten werden gemeinhin als „sensible“ Daten bezeichnet, vgl. Erwägungsgrund 10 S. 5.532 Art. 9 Abs. 1 DS-GVO untersagt die Verarbeitung sensibler Daten. Zu den sensiblen Daten gehören nach dem Wortlaut der Norm „personenbezogene Daten, aus denen die rassische und ethnische Herkunft, politische Meinungen, religiöse oder weltanschauliche Überzeugungen oder die Gewerkschaftszugehörigkeit [hervorgeht], sowie [...] genetische Daten, biometrische Daten zur eindeutigen Identifizierung einer natürlichen Person, Gesundheitsdaten oder Daten zum Sexualleben oder der sexuellen Orientierung einer natürlichen Person." Teleologisch ist in Art. 9 Abs. 1 DS-GVO unter anderem ein (in-

528 Frenzel in: Paal/Pauly (Hrsg.), Art. 9 DS-GVO Rn. 1; Schneider, ZD 2017, 303, 304 kritisiert diese in der DS-GVO angelegte Unterscheidung rechtspolitisch.

529 Siehe zu Art. 6 DS-GVO bereits zuvor auf S. $124 \mathrm{ff}$.

530 Kühling/Klar/Sackmann, Datenschutzrecht, Rn. 359, 439 ff.; Mester in: Taeger/ Gabel (Hrsg.), Art. 9 DS-GVO Rn. 2; Schneider, ZD 2017, 303, 304: „[...] die Erlaubnisse nach Art. 6 DS-GVO [werden] ausgehebelt." Zur umstrittenen Frage nach dem Verhältnis von Art. 6 Abs. 4 DS-GVO (Zweckänderung) und Art. 9 DS-GVO vgl. nur Schiff in: Ehmann/Selmayr (Hrsg.), Art. 9 DS-GVO Rn. 10 f. m.w.N.

531 Martini/Kienle, JZ 2019, 235, 239.

$532 \mathrm{Zu}$ dieser und anderen geläufigen Bezeichnungen vgl. Albers/Veit in: BeckOK Datenschutzrecht, Art.9 DS-GVO Rn. 7 m.w.N. sowie (kurz) Frenzel a.a.O. (Fn. 528). 
formationelles) Diskriminierungsverbot und damit eine normative Konkretisierung von Art. 21 Abs. 1 GRCh zu sehen. ${ }^{533}$

Für die Zugangsgewährung gegenüber den Erben folgt daraus, dass diese (zumindest auch) am Maßstab von Art. 9 DS-GVO zu messen ist, sofern mit dem Zugang zum Erblasseraccount die Möglichkeit zur Einsicht in sensible Daten der Kommunikationspartner einhergeht. ${ }^{534}$ Hier ist zwischen verschiedenen Internetdiensten zu unterscheiden. Etwa im Falle von Dating-Portalen muss mit Sicherheit davon ausgegangen werden, dass der Account des Erblassers (auch) sensible Daten der Kommunikationspartner enthält. Allerdings wird in der Literatur zurecht auf den weiten Anwendungsbereich von Art. 9 DS-GVO verwiesen, der sich nicht zuletzt aus dem Merkmal des „Hervorgehens“ ergibt. ${ }^{535}$ Frenzel nennt insoweit das Beispiel der Muttersprache, aus der die ethnische Herkunft als ein sensibles Datum im Sinne von Art. 9 Abs. 1 DS-GVO hervorgehen kann. ${ }^{536}$ Legt man diesen Maßstab zugrunde, können Angaben in beruflichen Netzwerken wie Xing oder LinkedIn sensible Daten darstellen. Demnach ist nicht nur bei Nutzerkonten in Dating-Portalen mit sensiblen Daten (der Kommunikationspartner) zu rechnen. ${ }^{537} \mathrm{Ob}$ der Anwendungsbereich von Art. 9 DS-GVO eröffnet ist, muss in jedem Einzelfall geprüft werden.

533 Weichert in: Kühling/Buchner (Hrsg.), Art. 9 DS-GVO Rn. 2, 15 f. m.w.N.; dazu und zu weiteren durch Art.9 DS-GVO konkretisierte Freiheitsgewährleistungen, vgl. Albers/Veit in: BeckOK Datenschutzrecht, Art.9 DS-GVO Rn. 4 mit Verweis auf Marsch, Das europäische Datenschutzgrundrecht, S. $90 \mathrm{ff}$.

534 BGH NJW 2018, 3178 Rn. 84, 93 sieht im Falle eines Facebook-Accounts zwar sensible Daten betroffen, erwähnt Art. 9 DS-GVO indessen nicht; zu Recht sehr kritisch deshalb Martini/Kienle, JZ 2019, 235, $239 \mathrm{f}$.

535 Schiff in: Ehmann/Selmayr (Hrsg.), Art. 9 DS-GVO Rn. 4 f.; Mester in: Taeger/ Gabel (Hrsg.), Art. 9 DS-GVO Rn. 6; kritisch zur Rechtslage Schneider, ZD 2017, 303, $303 \mathrm{ff}$.

536 Frenzel in: Paal/Pauly (Hrsg.), Art. 9 DS-GVO Rn. 8; den Verarbeitungskontext betont auch Mester in: Taeger/Gabel (Hrsg.), Art. 9 DS-GVO Rn. 6.

537 Für Facebook so bereits die Nachweise in Fn. 534. 
bb) Die Ausnahmetatbestände aus Art. 9 Abs. 2 DS-GVO

Art. 9 Abs. 2 DS-GVO zählt abschließend die Ausnahmetatbestände auf, welche aus dem grundsätzlichen Verarbeitungsverbot des Art. 9 Abs. 1 DSGVO herausführen. ${ }^{538}$

In Art. 9 Abs. 2 lit. a) DS-GVO ist als erster Ausnahmetatbestand - und insofern vergleichbar mit dem Katalog des Art. 6 Abs. 1 DS-GVO - die Einwilligung der betroffenen Person vorgesehen. Im Kontrast zur allgemeinen Einwilligung nach Art. 6 Abs. 1 S. 1 lit. a), Art. 7 DS-GVO sind indes erhöhte Anforderungen zu beachten. ${ }^{539}$ Insbesondere setzt Art. 9 Abs. 2 lit. a) DS-GVO eine ausdrückliche und demnach nicht lediglich konkludent erteilte Einwilligung voraus. ${ }^{540}$ Wie gesehen, erscheint es bereits zweifelhaft, dass die Kommunikationspartner bei Vertragsschluss mit dem Provider oder später in die Zugangsgewährung gegenüber den Erben im Sinne von Art. 6 Abs. 1 S. 1 lit. a) DS-GVO wirksam eingewilligt haben. ${ }^{541}$ Die Prüfung des Vorliegens einer Einwilligung bleibt zwar eine Frage des Einzelfalls, gleichwohl dürften sich die genannten Zweifel aber erst recht im Rahmen von Art. 9 Abs. 2 lit. a) DS-GVO auswirken. ${ }^{542}$

Weiterhin ist Art. 9 Abs. 2 lit e) DS-GVO zu beachten, wonach das Verbot aus Abs. 1 nicht gilt, soweit sich die Verarbeitung auf sensible Daten bezieht, „die die betroffene Person offensichtlich öffentlich gemacht hat. "543 Die Adressaten der Veröffentlichung durch den Betroffenen dürfen dabei aber nicht lediglich einem individuell bestimmten Personenkreis

538 Petri in: NK-Datenschutzrecht, Art. 9 DS-GVO Rn. 2; Schiff in: Ehmann/Selmayr (Hrsg.), Art. 9 DS-GVO Rn. 8; Frenzel in: Paal/Pauly (Hrsg.), Art. 9 DS-GVO Rn. 1.

539 Mester in: Taeger/Gabel (Hrsg.), Art. 9 DS-GVO Rn. 18; Schneider, ZD 2017, 303, 308, hingegen will im Wege einer weiten Auslegung des Einwilligungstatbestands eine zu starke Behinderung der Verarbeitung verhindern; dazu Albers/ Veit in: BeckOK Datenschutzrecht, Art. 9 DS-GVO Rn. 49.

540 Mester a.a.O. (Fn. 539); Schiff in: Ehmann/Selmayr (Hrsg.), Art. 9 DS-GVO Rn. 33; Petri in: NK-Datenschutzrecht, Art. 9 DS-GVO Rn.33; Frenzel in: Paal/ Pauly (Hrsg.), Art. 9 DS-GVO Rn. 21; nach Albers/Veit in: BeckOK Datenschutzrecht, Art. 9 DS-GVO Rn. 50 f. ist auch „ein gesteigertes Maß an Bestimmtheit erforderlich sowie die Nennung der betroffenen Daten und des Verwendungszwecks."

541 Siehe hierzu bereits zuvor auf S. $124 \mathrm{f}$.

542 Ebenso zweifelnd Martini/Kienle, JZ 2019, 235, 240.

$543 \mathrm{Zu}$ den Voraussetzungen von lit. e) siehe Petri in: NK-Datenschutzrecht, Art. 9 DS-GVO Rn. 57 ff.; Mester in: Taeger/Gabel (Hrsg.), Art. 9 DS-GVO Rn. 25; Schiff in: Ehmann/Selmayr (Hrsg.), Art. 9 DS-GVO Rn. 45 f. 
angehören. Vielmehr muss es sich um eine Mitteilung an die Allgemeinheit handeln. ${ }^{544} \mathrm{Im}$ Kontext sozialer Medien ist folglich zwischen öffentlich geteilten Informationen, wie in der Regel einem Tweet auf Twitter oder einem Lebenslauf bei LinkedIn, und beispielsweise Privatnachrichten zu unterscheiden, die nicht im Sinne von lit. e) veröffentlicht sind. ${ }^{545}$ Weiter verengt wird der Ausnahmetatbestand aus lit.e) durch das Merkmal „offensichtlich“, das einen eindeutigen, bewussten Willensakt des Betroffenen bei der Veröffentlichung voraussetzt. ${ }^{546}$ Zumindest die Gewährung des uneingeschränkten Zugangs zum Erblasseraccount lässt sich nach alledem nicht auf Art. 9 Abs. 2 lit e) DS-GVO stützen, wenn und soweit damit ein Zugriff auf in den Privatnachrichten enthaltene sensible Daten der Kommunikationspartner einhergeht.

Schließlich erlaubt Art. 9 Abs. 2 lit.f) DS-GVO „die Verarbeitung [...] zur Geltendmachung, Ausübung oder Verteidigung von Rechtsansprüchen" soweit erforderlich. Dieser Ausnahmetatbestand ist indes nicht mit der Abwicklung eines Vertragsverhältnisses im Sinne von Art. 6 Abs. 1 S. 1 lit. b) Var. 1 DS-GVO gleichzusetzen. ${ }^{547}$ Die eindeutige begriffliche Differenzierung und die Systematik sprechen dafür, dass Art. 9 Abs. 2 lit. f) DSGVO nur im Falle eines rechtlichen Konflikts, nicht jedoch zur regulären Vertragsabwicklung und -erfüllung herangezogen werden kann. ${ }^{548}$ Insbesondere sofern die Verarbeitung sensibler Daten selbst die vertragliche Erfüllungshandlung darstellt, bedarf es folglich eines eigenen Ausnahmetatbestands namentlich in Form der Einwilligung nach Art. 9 Abs. 2 lit. a) DS-GVO. ${ }^{549}$ Ebenso verhält es sich bei der Zugangsgewährung gegenüber den Erben, da der Provider damit den im Erbgang übergegangenen vertraglichen Zugangsanspruch der Erben erfüllt. Diese Zugangsgewährung unterschiedslos auf Art. 9 Abs. 2 lit. a) DS-GVO wie bereits auf Art. 6 Abs. 1

544 Mester in: Taeger/Gabel (Hrsg.), Art. 9 DS-GVO Rn. 25; Schiff in: Ehmann/ Selmayr (Hrsg.), Art. 9 DS-GVO Rn. 45.

545 Schiff a.a.O. (Fn. 544); Petri in: NK-Datenschutzrecht, Art. 9 DS-GVO Rn. 58; Martini/Kienle, JZ 2019, 235, 240; Spindler/Dalby in: Spindler/Schuster (Hrsg.), Recht der elektronischen Medien, Art. 9 DS-GVO Rn. 14; Frenzel in: Paal/Pauly (Hrsg.), Art. 9 DS-GVO Rn. 67 zufolge „transzendieren [geschlossenen Gruppen in sozialen Medien] die klassische Dichotomie von öffentlich/nicht-öffentlich.“

546 Schiff a.a.O. (Fn. 544); Frenzel in: Paal/Pauly (Hrsg.), Art. 9 DS-GVO Rn. 66.

547 Albers/Veit in: BeckOK Datenschutzrecht, Art.9 DS-GVO Rn. 45; zu Art. 6 Abs. 1 S. 1 lit. b) Var. 1 DS-GVO siehe S. 126 ff.

548 Schiff in: Ehmann/Selmayr (Hrsg.), Art. 9 DS-GVO Rn. 48; Schneider, ZD 2017, 303, 305 a.E; ähnlich auch Weichert in: Kühling/Buchner (Hrsg.), Art. 9 DS-GVO Rn. 84.

549 Schiff in: Ehmann/Selmayr (Hrsg.), Art. 9 DS-GVO Rn. 48. 
S. 1 lit. b) Var. 1 DS-GVO zu stützen, hieße die Systematik der DS-GVO und den darin angelegten besonderen Schutz sensibler Daten zu unterlaufen.

\section{cc) Zwischenergebnis zu c)}

Ob der Anwendungsbereich von Art. 9 DS-GVO eröffnet und dadurch das Verarbeitungsverbot aus Abs. 1 berufen ist, muss ebenso wie die Frage, inwieweit ein etwaig einschlägiger Ausnahmetatbestand aus Abs. 2 in Betracht kommt, im Einzelfall geprüft und kann hier nicht abstrakt für unterschiedliche Internetdienste und Nutzungsverhältnisse beurteilt werden. Indessen lassen sich zwei zentrale Punkte festhalten, die für den digitalen Nachlass von Bedeutung sind: Erstens ergibt sich aus der intensiven Nutzung von Internetdiensten durch viele Privatpersonen in Kombination mit dem breit formulierten Wortlaut des Anwendungsbereichs („hervorgehen"), dass Art. 9 DS-GVO nicht nur in seltenen Ausnahmefällen einschlägig sein dürfte. ${ }^{550}$ Zweitens hat sich gezeigt, dass die Zugangsgewährung gegenüber den Erben erheblichen Schwierigkeiten begegnet, sobald durch das Vorliegen sensibler Daten der Kommunikationspartner ebendieser Anwendungsbereich von Art. 9 Abs. 1 DS-GVO eröffnet ist. Denn Art. 9 Abs. 2 DS-GVO enthält keinen allgemeinen Abwägungstatbestand, der mit Art. 6 Abs. 1 S. 1 lit.f) DS-GVO vergleichbar wäre. ${ }^{551}$ Über die explizit in Art. 9 Abs. 2 DS-GVO genannten Ausnahmen hinaus gibt es daher keine Möglichkeit, durchaus berechtigte Interessen etwa der Erben zu berücksichtigen. Das nationale (Erb-)Recht hat gegenüber der Wertung des in seiner Anwendung vorrangigen unionalen Datenschutzrechts insofern zurückzustehen. ${ }^{552}$

550 Umso bemerkenswerter ist es, dass der BGH Art. 9 DS-GVO unerwähnt lässt, obgleich er explizit (auch) sensible Daten betroffen sieht, vgl. BGH NJW 2018, 3178 Rn. 84, 93; sehr kritisch dazu Martini/Kienle, JZ 2019, 235, 239 f.; zustimmend hingegen Härting/Dag, K\&R 2018, 638, 639.

551 Mester in: Taeger/Gabel (Hrsg.), Art. 9 DS-GVO Rn. 2 sieht Art. 6 Abs. 1 S. 1 lit. f) DS-GVO durch die Sperrwirkung des lex specialis Grundsatzes explizit ausgeschlossen.

552 So Martini/Kienle, JZ 2019, 235, 239; rechtpolitische Kritik wegen mangelnder Praxistauglichkeit von Art. 9 DS-GVO allgemein übt Schneider, ZD 2017, 303. 
d) Zwischenergebnis zu 1.

Die DS-GVO schützt (lediglich) die personenbezogenen Daten von lebenden Personen. Das unionale Datenschutzrecht kann nicht für den vereinzelt geforderten Schutz der Privatsphäre des Erblassers (vor seinen eigenen Erben) herangezogen werden. Da mit dem Zugang der Erben zum Account des Erblassers neben dessen Daten auch diejenigen seiner Kommunikationspartner zwangsläufig verarbeitet werden, ist die DS-GVO allerdings zum Schutz der (lebenden) Kommunikationspartner zur Anwendung berufen. Während das Erbrecht nur die Extrempositionen von umfassender Vererbbarkeit beziehungsweise von Unvererbbarkeit der nutzungsvertraglichen Stellung des Erblassers kennt, erlaubt die DS-GVO vermittelnde Positionen. Einen Ansatzpunkt dafür bietet zum einen das Merkmal der Erforderlichkeit, durch welches die Anonymisierung oder zumindest Pseudonymisierung (von Teilen) der Inhalte des Erblasseraccounts verlangt werden kann. Zum anderen ermöglicht ein etwaiges Widerspruchsrecht der Betroffenen die Unterscheidung zwischen mehreren Kommunikationspartnern, die in ihren Privatheitsinteressen unterschiedlich intensiv berührt sind. Durch diese beiden Ansatzpunkte sowie wegen der ohnehin einzelfallbezogenen Beurteilung (und im Falle von Art. 6 Abs. 1 S. 1 lit. f) DS-GVO Abwägung), welche die DS-GVO voraussetzt, ist das Datenschutzrecht besser als das Erbrecht geeignet, um sachgerechte Ergebnisse auch und gerade vor dem Hintergrund der multipolaren Rechtsbeziehungen im digitalen Nachlass zu ermitteln.

Erheblich höhere Hürden bei der Zugangsgewährung und damit verbunden eine Einbuße bei der soeben festgestellten Flexibilität der DS-GVO sind zu verzeichnen, sobald der Erblasseraccount sensible Daten der Kommunikationspartner im Sinne von Art. 9 Abs. 1 DS-GVO enthält. Abgesehen von einer etwaigen Einwilligung der Betroffenen nach Art. 9 Abs. 2 lit. a DS-GVO, die einzuholen dem Verantwortlichen zu raten ist, erfasst keiner der Ausnahmetatbestände in Art. 9 Abs. 2 DS-GVO rechtssicher die vorliegende Konstellation. Auf Rechtssicherheit sind nicht zuletzt die Provider als Verantwortliche indes dringend angewiesen, seit mit Einführung der DS-GVO eine erhebliche Verschärfung des Bußgeldrahmens bei Datenschutzverstößen einhergegangen ist. ${ }^{553}$

553 Dazu Frenzel in: Paal/Pauly (Hrsg.), Art. 83 DS-GVO Rn. 1 sowie Faust/Spittkal Wybitul, ZD 2016, 120, 120 ff.; Boehm in: NK-Datenschutzrecht, Art. 83 DSGVO Rn. 3 vergleicht den Bußgeldrahmen mit dem des europäischen Kartellrechts; zu Kriterien und Praxis der Bußgeldzumessung vgl. Paal, RDV 2020, 57, 
2. Fernmeldegeheimnis gem. $₫ 88$ TKG

Während das verfassungsrechtliche Fernmeldegeheimnis aus Art. 10 Abs. 1 Var. 3 GG lediglich den Staat unmittelbar verpflichtet, ${ }^{554}$ hat der einfache Gesetzgeber mit $\$ 88$ TKG eine Norm geschaffen, die auch private Anbieter von Telekommunikationsdiensten zu Adressaten des Fernmeldegeheimnisses macht. ${ }^{555}$ Mit Blick auf Gegenstand, Inhalt und Reichweite des Schutzbereichs entsprechen Art. 10 GG und $\$ 88$ TKG einander weitgehend. ${ }^{556}$ Der sachliche Schutzbereich des Fernmeldegeheimnisses erfasst die Vertraulichkeit der Individualkommunikation im Fernmeldeverkehr. ${ }^{557}$ Fraglich ist, ob das Fernmeldegeheimnis damit der Durchsetzung des auf die Erben übergegangenen Rechts auf Zugang zum Erblasseraccount entgegensteht. Hier ist zunächst zu prüfen, ob und inwieweit die Anbieter von Kommunikations- und Informationsdiensten zur Einhaltung von $\$ 88$ TKG verpflichtet sind (dazu a). Danach ist die Zugangsgewährung gegenüber den Erben am Tatbestand von $\$ 88$ Abs. 3 TKG zu messen (dazu b).

$57 \mathrm{ff}$; neben das Bußgeld- tritt ein Haftungsrisiko auf Schadensersatz gem. Art. 82 DS-GVO, vgl. dazu Paal, MMR 2020, 14, 14 ff.; von „Verunsicherung“ der großen Anbieter berichtet Pruns, ErbR 2019, 456, 457, der ferner auf $\$ 206$ StGB hinweist.

554 Hermes in: Dreier (Hrsg.), Art. 10 GG Rn. 50; zur Privatisierungsgeschichte der Deutschen Bundespost in diesem Zusammenhang vgl. Ogorek in: BeckOK GG, Art. 10 Rn. 12, 12.1 m.w.N.; s. aber Mayen in: DAV-Stellungnahme Nr. 34/2013, S. 67 ff., der breiter argumentiert mit Verweis auf BVerfGE 128, 226, $249 \mathrm{f}$. FRAPORT; ders., ZHR 182 (2018), 1, $6 \mathrm{f}$.

555 Kühling/Schall/Biendl, Telekommunikationsrecht, Rn.76; anschaulich KG Berlin, ZD 2017, 386 Rn. 66-68; Mayen in: Scheurle/Mayen (Hrsg.), $\$ 88$ TKG Rn. 1; s. aber ders. a.a.O (Fn. 554); Graulich in: Arndt/Fetzer/Scherer/Graulich (Hrsg.), $\$ 88$ TKG Rn. 3 mit Hinweis auf $\$ 206$ StGB, der ebenfalls Private adressiert und die Verletzung des Fernmeldegeheimnisses unter Strafe stellt.

556 Mayen in: Scheurle/Mayen (Hrsg.), $\mathbb{8} 88$ TKG Rn. 3; Eckhardt in: Spindler/Schuster (Hrsg.), Recht der elektronischen Medien, $\mathbb{} 88$ TKG Rn. 4 m.w.N.; KG Berlin a.a.O. (Fn. 555); zum Schutzbereich von Art. 10 GG und $\$ 88$ TKG vgl. Bock in: Beck'scher TKG-Kommentar, $\mathbb{8} 88$ TKG Rn. 4 ff. respektive Rn. 12 ff.

557 Gusy in: v. Mangoldt/Klein/Starck, Art. 10 GG Rn. 59; Hermes in: Dreier (Hrsg.), Art. 10 GG Rn. 36; Kühling/Schall/Biendl, Telekommunikationsrecht, Rn.69; KG Berlin a.a.O. (Fn. 555). 
a) Verpflichtung des Providers durch $\$ 88 \mathrm{TKG}$

Die Eigenschaft als Adressat des Fernmeldegeheimnisses kann sich für den Anbieter von Kommunikations- und Informationsdiensten aus $₫ 88$ Abs. 2 S. 1 TKG (dazu aa) oder aus $\$ 7$ Abs. 3 S. 2 TMG (dazu bb) ergeben.

aa) Verpflichtung als Diensteanbieter im Sinne des TKG

Den originären Anwendungsbereich des einfachrechtlichen Fernmeldegeheimnisses bestimmt zunächst $\$ 88$ Abs. 2 S. 1 TKG, wonach jeder „Diensteanbieter" zur Wahrung des Fernmeldegeheimnisses verpflichtet ist. ${ }^{558}$ Nach der Legaldefinition des $\$ 3$ Nr. 6 TKG ist „'Diensteanbieter ' jeder, der ganz oder teilweise geschäftsmäßig Telekommunikationsdienste erbringt oder an der Erbringung solcher Dienste mitwirkt." Telekommunikationsdienste wiederum werden in $\$ 3 \mathrm{Nr} .24 \mathrm{TKG}$ definiert als, ,in der Regel gegen Entgelt erbrachte Dienste, die ganz oder überwiegend in der Übertragung von Signalen über Telekommunikationsnetze bestehen, einschließlich Übertragungsdienste in Rundfunknetzen."

Problematisch ist vorliegend, dass sich die Anbieter von Kommunikations- und Informationsdiensten bei sogenannten „Over-the-top“-Diensten der fremden Signalübertragung durch Telekommunikationsunternehmen bedienen, nicht aber selbst Signale übertragen. ${ }^{59}$ Versendet ein Nutzer beispielsweise eine Nachricht oder einen sonstigen Inhalt über WhatsApp, wird diese Nachricht - anders etwa als die über das Vodafone-Netz übermittelte SMS eines Vodafone-Kunden - nicht über WhatsApp-eigene Telekommunikationsnetze, sondern über das offene Internet übermittelt. ${ }^{560}$ Bislang wurde darüber gestritten, ob sich der Anbieter des Kommunikationsdienstes (im Beispiel WhatsApp) die Signalübertragung der Telekom-

558 Zum Adressatenkreis nach $₫ 88$ Abs. 2 S. 1 TKG vgl. Graulich in: Arndt/Fetzer/ Scherer/Graulich (Hrsg.), $\$ 88$ TKG Rn. 75; Mayen in: Scheurle/Mayen (Hrsg.), $\$ 88$ TKG Rn. 53 ff.; Bock in: Beck’scher TKG-Kommentar, $\$ 88$ TKG Rn. 22 ff.; Eckhardt in: Spindler/Schuster (Hrsg.), Recht der elektronischen Medien, $\mathbb{8} 88$ TKG Rn. $26 \mathrm{ff}$.

559 Instruktiv zu Begriff und Funktionsweise unterschiedlicher OTT-Dienste vgl. Grünwald/Nüßing, MMR 2016, 91, 91 ff.; ferner Schuster, CR 2016, 173, 174.

560 Speziell zum Beispiel von WhatsApp und seiner Funktionsweise in diesem Zusammenhang, vgl. Schneider, ZD 2014, 231, 232 m.w.N. 
munikationsunternehmen zurechnen lassen muss und sich dadurch selbst ebenfalls als Adressat des TKG qualifiziert. ${ }^{561}$

Die aufgeworfene Frage ist durch die dem TKG zugrunde liegende und hier entscheidende Vorschrift des Art.2 lit.c) der Rahmenrichtlinie 2002/21/EG europarechtlich determiniert. Der EuGH ist der vorgenannten extensiven Lesart im Sinne einer Zurechnung jüngst klar entgegengetreten. ${ }^{562}$ In einem Urteil zu Googles Webmail-Dienst Gmail nimmt der EuGH eine technisch geprägte Begriffsbestimmung vor und sieht keine „ganz oder überwiegend“ in der Übertragung von Signalen bestehende Tätigkeit aufseiten von Gmail. ${ }^{563} \mathrm{Nach}$ diesem Urteil, das auf Vorlage des OVG NRW ${ }^{564}$ erging, dürfte zumindest die Einbeziehung aller OTT-Dienste in das Regelungsregime des TKG nunmehr kaum noch vertretbar sein. ${ }^{565}$ Allerdings wird sich zukünftig aus dem bis 21. Dezember 2020 umzusetzenden neuen Europäischen Kodex für die elektronische Kommunikation (EKEK-RL) wahrscheinlich eine Einbeziehung von OTT-Kommunikationsdiensten in den unionalen Regelungsrahmen ergeben, vgl. Art. 2 Nr. 4 lit. b), Nr. 5 EKEK-RL. ${ }^{566}$

561 Ausführlich dazu Bulowski, Regulierung von Internetkommunikationsdiensten, S. 55 ff. m.w.N.; Spindler in: Schmitz/Spindler (Hrsg.), $\$ 1$ TMG Rn. 26 ff.; für eine weite Auslegung des TKG in diesem Sinne Kühling/Schall, CR 2015, 641, 641 ff. und dies., CR 2016, 185, 185 ff.; hingegen für eine enge Auslegung unter Ausschluss der Anwendbarkeit auf OTT-Dienste etwa Schuster, CR 2016, 173, $173 \mathrm{ff} . ;$ Gersdorf, K\&R 2016, 91, $91 \mathrm{ff} . ;$ differenzierend Grünwald/Nüßing, MMR 2016, 91, 91 ff.; Schneider, ZD 2014, 231, 235 f.; im Kontext des digitalen Nachlasses zu diesem Streit noch Fußeder, Soziale Netzwerke im Nachlass, S. 153 ff. sowie Thiesen, Daten in der Erbmasse, S. 185 ff.; BGH NJW 2018, 3178 Rn. 56 lässt die Frage explizit offen.

562 EuGH Urteil vom 13.6.2019 - C-193/18 - MMR 2019, 514 mit Anm. von Spies; dazu auch Schumacher, K\&R 2019, 490, $490 \mathrm{f}$.

563 EuGH MMR 2019, 514 Rn. 35 ff.; Ludwigs/Huller, NVwZ 2019, 1099, 1099 f.

564 OVG NRW MMR 2018, 552, $552 \mathrm{ff}$.

565 Zum Ganzen Martini in: BeckOK Informations- und Medienrecht, $\mathbb{1}$ TMG Rn.13aff.; instruktiv zur Unterscheidung zwischen OTT-Kommunikationsdiensten (wie Gmail und WhatsApp), die nicht unter das TKG fallen, und OTT-0-Diensten (wie SkypeOut), die nach EuGH Urteil vom 5.6.2019 C-142/18 - MMR 2019, 517 von der Rahmenrichtlinie 2002/21/EG erfasst sind, vgl. Ludwigs/Huller, NVwZ 2019, 1099, 1101; Schumacher, K\&R 2019, 490, $490 \mathrm{f}$.

566 Ludwigs/Huller, NVwZ 2019, 1099, 1101; Martini in: BeckOK Informations- und Medienrecht, $\mathbb{1}$ TMG Rn.13f; Schumacher, K\&R 2019, 490, 491; Spies, MMR 2019, 516, 517 (auch) zu den mitgliedstaatlichen Umsetzungsbestrebungen in Deutschland. 
bb) Verpflichtung als Diensteanbieter im Sinne des TMG

Ungeachtet der strittigen und dynamischen Rechtslage im Telekommunikationsrecht ergibt sich die Geltung von $\$ 88$ TKG für die Anbieter von Informations- und Kommunikationsdiensten jedenfalls (auch) aus dem Verweis in $\$ 7$ Abs. 3 S. 2 TMG. ${ }^{567}$ Danach haben Diensteanbieter im Sinne

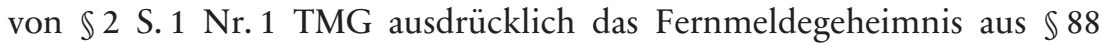
TKG zu wahren. ${ }^{568}$ Diensteanbieter im Sinne des TMG ist, wer „eigene oder fremde Telemedien zur Nutzung bereithält oder den Zugang zur Nutzung vermittelt," vgl. $\$ 2$ S. 1 Nr. 1 TMG. Telemedien wiederum sind nach $₫ 1$ Abs. 1 S. 1 TMG ,alle elektronischen Informations- und Kommunikationsdienste, soweit sie nicht Telekommunikationsdienste nach $\mathbb{3}$ Nr. 24 TKG [...], telekommunikationsgestützte Dienste nach $\$ 3$ Nr. 25

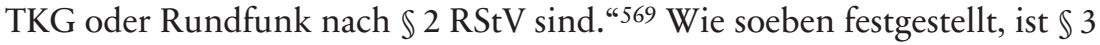
Nr. 24 TKG hier de lege lata nicht erfüllt. ${ }^{570}$ Das Vorliegen von telekommunikationsgestützten Diensten und Rundfunk kann ebenfalls ausgeschlossen werden. ${ }^{571}$ Damit sind die drei negativen Voraussetzungen des Telemedienbegriffs erfüllt. Die Anbieter sozialer Medien vermitteln auf elektronischem Wege, insbesondere über das Internet, Information und Kommunikation. Nach alledem stellen die Anbieter von Informationsund Kommunikationsdiensten somit Telemedien dar. ${ }^{572}$

567 So Martini/Kienle, JZ 2019, 235, 236; anders noch zur Rechtslage vor der 3. TMG-Novelle gem. $\$ 7$ Abs. 2 S. 3 TMG a.F. Martini, JZ 2012, 1145, 1148; wie hier auch Mayen, AnwBl Online 2018, 466, 467; vgl. ferner ders., in: Scheurle/ Mayen (Hrsg.), $\$ 88$ TKG Rn. 60; a.A., der sachliche Schutzbereich sei nicht eröffnet, Seidler, Digitaler Nachlass, S. 138 f.

568 Vgl. hierzu Paal in: BeckOK Informations- und Medienrecht, $\$ 7$ TMG Rn. 71; Hoffmann/Volkmann in: Spindler/Schuster (Hrsg.), Recht der elektronischen Medien, $\mathbb{} 7$ TMG Rn. 43; noch zu $₫ 7$ Abs. 2 S. 3 TMG a.F. ebenfalls MüllerBroich in: NK-TMG, $\$ 7$ Rn. 10.

569 Zum Begriff der Telemedien s. Spindler in: Schmitz/Spindler (Hrsg.), $\$ 1$ TMG Rn. 8 ff. sowie Martini in: BeckOK Informations- und Medienrecht, $\mathbb{1} 1$ TMG Rn. 8 ff.

570 Siehe dazu zuvor auf S. $149 \mathrm{f}$.

571 Zum Begriff des $\$ 3$ Nr. 25 TKG vgl. Ricke in: Spindler/Schuster (Hrsg.), Recht der elektronischen Medien, $\mathbb{3}$ TKG Rn. 51; Ditscheid in: Beck'scher TKG-Kommentar, $\$ 3$ TKG Rn. $80 \mathrm{ff}$.

572 Für Twitter so etwa Engeler, MMR 2017, 651, 652; für Facebook Rockstroh, MMR 2013, 627, 628 sowie Martini, JZ 2012, 1145, 1148; i.Ü. für WebmailDienste Martini ebd.; weitere Beispiele bei Ricke in: Spindler/Schuster (Hrsg.), Recht der elektronischen Medien, $\mathbb{} 1$ TMG Rn. 12. 
cc) Zwischenergebnis zu a)

Die Anbieter von Informations- und Kommunikationsdiensten sind zur Wahrung des Fernmeldegeheimnisses aus $\$ 88$ TKG verpflichtet. Das folgt de lege lata zwar nicht aus $\mathbb{} 88$ Abs. 2 S. 1 TKG, aber aus $\mathbb{} 7$ Abs. 3 S. 2 TMG. De lege ferenda ist die Entwicklung der mitgliedstaatlichen Umsetzung von Art. 2 Nr. 4 lit.b), Nr. 5 EKEK-RL in nationales Recht zu beobachten. Während die Geltung des Fernmeldegeheimnisses für die Anbieter von Informations- und Kommunikationsdiensten dem Grunde nach (wohl) fortbestehen wird, könnte dieses Ergebnis nach einer TKG-Novelle rechtlich anders herzuleiten sein.

\section{b) Tatbestand von $\$ 88$ Abs. $3 \mathrm{TKG}$}

Die Inhalte des Erblasseraccounts, insbesondere die davon umfassten Privatnachrichten, fallen in den sachlichen Schutzbereich des Fernmeldegeheimnisses, solange und soweit sie im Herrschaftsbereich - also insbesondere auf den Servern - des Providers abgespeichert sind. ${ }^{573}$ Das gilt unabhängig davon, ob der Adressat den Inhalt der Nachrichten bereits zur Kenntnis genommen hat beziehungsweise ob die Nachrichten auf dem Server zwischen- oder endgespeichert sind. ${ }^{574}$ Bei der Nutzung sozialer Medien verbleiben die Accountinhalte regelmäßig auf dem Server des Providers. Einer Differenzierung wie bei E-Mails zwischen Webmail-Diensten (Speicherung auf dem Server des Providers) und endgerätebasierten EMail-Programmen (Speicherung auf dem Endgerät des Nutzers) bedarf es nicht. Demnach unterfällt der Inhalt eines Social-Media-Accounts dem

573 Martini/Kienle, JZ 2019, 235, 235; zum Schutzbereich vgl. Graulich in: Arndt/ Fetzer/Scherer/Graulich (Hrsg.), $\$ 88$ TKG Rn. 21; Bock in: Beck'scher TKGKommentar, $\mathbb{} 88$ TKG Rn. 12.

574 So explizit BVerfGE 124, 43, 55 zu Art. 10 Abs. 1 GG. Nach Mayen in: Scheurle/ Mayen (Hrsg.), $\mathbb{8} 88$ TKG Rn. 27 gelten diese Maßstäbe „auch für $\$ 88$ TKG, der den Zweck verfolgt, das im Staat-Bürger-Verhältnis unmittelbar geltende Fernmeldegeheimnis des Art. 10 GG auf das Horizontalverhältnis zwischen privatem Diensteanbieter und privatem Nutzer zu übertragen." Vgl. ferner ders., AnwBl Online 2018, 466, $467 \mathrm{f}$. sowie bereits ders. in: DAV-Stellungnahme Nr. 34/2013, S. $71 \mathrm{ff}$;; Kühling/Schall/Biendl, Telekommunikationsrecht, Rn. $71 \mathrm{ff}$.; bis zur Klarstellung durch das BVerfG noch a.A. BGH NJW 2009, 1828, 1828. 
Fernmeldegeheimnis. ${ }^{575}$ Geschützt sind dabei sowohl der Empfänger als auch der Absender der jeweiligen Privatnachricht. ${ }^{576}$

Nach $₫ 88$ Abs. 3 S. 1 TKG ist es dem Provider als Normadressaten untersagt, „sich oder anderen über das für die geschäftsmäßige Erbringung der Telekommunikationsdienste [...] erforderliche Maß hinaus Kenntnis vom Inhalt oder den näheren Umständen der Telekommunikation zu verschaffen." Umstritten ist, ob $₫ 88$ Abs. 3 S. 1 TKG damit dem Provider die Zugangsverschaffung gegenüber den Erben verbietet.

\section{aa) Herrschende Ansicht: Kein Verstoß gegen $\$ 88$ Abs. 3 S. 1 TKG}

Die überwiegende Ansicht in der Literatur ${ }^{577}$ und im Anschluss daran der $\mathrm{BGH}^{578}$ sehen in der Zugangsverschaffung zugunsten der Erben keinen Verstoß gegen $\ 88$ Abs. 3 S. 1 TKG. Während innerhalb dieser Meinung zwar mit Blick auf das Ergebnis Einigkeit herrscht, werden zu dessen Herleitung im Wesentlichen aber vier unterschiedliche Anknüpfungspunkte vorgeschlagen:

Einige Stimmen rekurrieren ausgehend von der Annahme, dass der $\mathrm{Zu}$ gangsanspruch grundsätzlich im Wege des Erbgangs auf die Erben übergeht, ${ }^{579}$ auf das vertragliche Pflichtenregime des Providers, um die TKGKonformität zu begründen: Die Zugangsverschaffung stelle die Erfüllung vertraglicher Pflichten des Providers dar und halte sich deshalb in dem „für die geschäftsmäßige Erbringung der Telekommunikationsdienste“ erforderlichen Maß der Kenntnisverschaffung. ${ }^{580}$ Das erforderliche Maß der Kenntnisverschaffung wird in der telekommunikationsrechtlichen Litera-

575 Mayen, AnwBl Online 2018, 466, 468 mit Verweis auf KG Berlin, ZEV 2017, 386 Rn. 86; Martini/Kienle, JZ 2019, 235, 235; Härting/Dag, K\&R 2018, 638, 639.

576 Graulich in: Arndt/Fetzer/Scherer/Graulich (Hrsg.), $\$ 88$ TKG Rn. 22; Bock in: Beck'scher TKG-Kommentar, $₫ 88$ TKG Rn. 19.

577 Ein guter Überblick zu den vier vertretenen Begründungsansätzen findet sich bei Herzog in: NK-Nachfolgerecht, Kap. 9 Rn. 59 ff. sowie bei dies./Pruns, Der digitale Nachlass in der Vorsorge- und Erbrechtspraxis, S. 82 ff.; für ausführliche Nachweise zu den vier Teilmeinungen siehe noch Fn. 580, 584, 587 und 592.

578 BGH NJW 2018, 3178 Rn. $56 \mathrm{ff}$.

579 Das entspricht auch der hier vertretenen Auffassung, vgl. dazu S. $88 \mathrm{ff}$.

580 So erstinstanzlich das LG Berlin ZEV 2016, 189, $192 \mathrm{f}$. mit Verweis auf Steiner/ Holzer, ZEV 2015, 262, 264; Pruns, NWB 2014, 2175, 2178; Thiesen, Daten in der Erbmasse, S. $201 \mathrm{f}$. 
tur allerdings zum einen eng und zum anderen technisch ausgelegt. ${ }^{581}$ Nach Graulich soll sich der Provider selbst nur Kenntnis verschaffen dürfen, wenn das für die Herstellung der Verbindung, die Beseitigung von Fehlerquellen oder für die Abrechnung der erbrachten Leistung erforderlich ist. ${ }^{582}$ Gemessen an diesem Maßstab ist eine Kenntnisverschaffung des Kommunikationsinhalts - insbesondere zugunsten Dritter - regelmäßig nicht erforderlich. ${ }^{583}$

Auf die Erforderlichkeit kommt es indes nicht an, wenn die Erben bereits keine „anderen“ sind im Sinne von $\ 88$ Abs. 3 S. 1 BGB. ${ }^{584}$ Nach Ansicht des BGH werde anstelle des Erblassers der Erbe als neuer Kontoberechtigter zum Teilnehmer der (fortlaufenden) Kommunikationsvorgänge. ${ }^{585}$ Demnach soll eine erbrechtlich bedingte Sukzession auch in die Stellung des Kommunikationsteilnehmers stattfinden. Dem wird entgegnet, dass „anderer“ jeder sei, der nicht an der Telekommunikation beteiligt war; der Beteiligtenbegriff wiederum sei lebensweltlich und nicht vertragsbeziehungsweise erbrechtlich zu beurteilen. ${ }^{586} \mathrm{Da}$ die Erben nicht selbst und persönlich am Kommunikationsvorgang beteiligt waren, seien sie „andere“ im Sinne von $\$ 88$ Abs. 3 S. 1 BGB.

Ein weiterer Ansatz zur Begründung der TKG-Konformität geht von einer entweder expliziten, konkludenten oder zumindest mutmaßlichen Einwilligung des Erblassers sowie seiner Kommunikationspartner in die

581 Graulich in: Arndt/Fetzer/Scherer/Graulich (Hrsg.), $\mathbb{} 88$ TKG Rn. 77 ff.; Bock in: Beck'scher TKG-Kommentar, $\mathbb{8} 88$ TKG Rn. 26.

582 Graulich in: Arndt/Fetzer/Scherer/Graulich (Hrsg.), $\$ 88$ TKG Rn.78; ähnlich auch Bock in: Beck'scher TKG-Kommentar, $\$ 88$ TKG Rn. 26.

583 Graulich in: Arndt/Fetzer/Scherer/Graulich (Hrsg.), $\$ 88$ TKG Rn. 79 f. (beachte aber Rn. 74); zum digitalen Nachlass hierzu KG Berlin, ZEV 2017, 386 Rn. 78 ff.; zweifelnd auch Kunz in: Staudinger, $\$ 1922$ BGB Rn. 629.

584 So BGH NJW 2018, 3178 Rn. 56 ff.; dem zustimmend Hoeren, MMR 2018, 749, 749 f. sowie Preuß, NJW 2018, 3146, 3146 f.; in diesem Sinne bereits zuvor Biermann, ZErb 2017, 210, 214 f.; Herzog/Pruns, Der digitale Nachlass in der Vorsorge- und Erbrechtspraxis, S. 86 f.; Herzog, ZErb 2017, 205, 208; Wüsthof, ErbR 2017, 508, 510; Steiner/Holzer, ZEV 2015, 262, 264; Budzikiewicz, AcP (218) 2018, 558, 585; Seidler, Digitaler Nachlass, S. 115; Bericht der Arbeitsgruppe „Digitaler Neustart“ der Konferenz der Justizministerinnen und Justizminister der Länder, S. 347, abrufbar a.a.O. (Fn. 43).

585 BGH NJW 2018, 3178 Rn. 60.

586 Martini/Kienle, JZ 2019, 235, 236; Mayen, AnwBl Online 2018, 466, 468 f.; Bock, AcP (217) 2017, 370, 406; Fußeder, Soziale Netzwerke im Nachlass, S. 211; KG Berlin, ZEV 2017, 386 Rn. 85. 
Zugangsgewährung gegenüber den Erben aus. ${ }^{587}$ Der Erblasser erteile bereits durch die Erbeinsetzung seine Einwilligung. Da beide Kommunikationsteilnehmer durch das Fernmeldegeheimnis geschützt werden, ${ }^{588}$ könne der Empfänger allein zwar nicht wirksam in den Zugriff auf die gem. $\$ 88$ TKG geschützten Daten einwilligen. ${ }^{589}$ Indes willigten auch die Kommunikationspartner (konkludent) in den Zugriff der Erben ein. ${ }^{590}$ Dieser Meinung wird zumindest in ihrer Pauschalität die Gefolgschaft von anderen Stimmen verweigert, die auf die Notwendigkeit einer einzelfallbezogenen Feststellung der Einwilligung hinweisen und zumindest für besonders sensible Korrespondenz keinen dahingehenden Willen der Kommunikationspartner erkennen wollen. ${ }^{591}$

Schließlich gibt es noch einen vierten Ansatz, der das gewünschte Ergebnis der TKG-Konformität gleichsam im Wege einer Gesamtbetrachtung unter Verweis auf das Rechtsinstitut der praktischen Konkordanz herleitet. ${ }^{592}$ Die Interessen der Erben und der Sukzessionsgedanke dürften angesichts des verfassungsrechtlich geschützten Erbrechts, vgl. Art. 14 Abs. 1 S. 1 Var. 2 GG, nicht durch die Beschränkung des Accountzugangs ausgehöhlt werden. Diesem Ansatz wird vorgeworfen, das Verfassungsrechtsinstitut „lediglich als ein Vehikel für eine rein vom Ergebnis her orientierte Betrachtung" zu gebrauchen. ${ }^{593}$ Denn unter praktischer Konkordanz sei nach ständiger Rechtsprechung des BVerfG der schonende Ausgleich kollidierender Grundrechtspositionen in dem Sinne zu verstehen, dass die jeweiligen Interessen der Beteiligten möglichst weitgehend wirksam wer-

587 Pruns, NWB 2014, 2175, 2183 ff.; ders., ZErb 2017, 217, 220 ff.; Herzog in: NKNachfolgerecht, Kap. 9 Rn. 68a; Herzog/Pruns, Der digitale Nachlass in der Vorsorge- und Erbrechtspraxis, S. 90 ff.; Fußeder, Soziale Netzwerke im Nachlass, S. 212 f.; Thiesen, Daten in der Erbmasse, S. 198 ff.; zumindest für den geschäftlichen Kontext so auch Ludyga, ZEV 2018, 1, 6.

588 Siehe dazu bereits die Nachweise in Fn. 576.

589 Bock in: Beck'scher TKG-Kommentar, $\$ 88$ TKG Rn. 19; problematisch insoweit der Bericht der Arbeitsgruppe „Digitaler Neustart“ der Konferenz der Justizministerinnen und Justizminister der Länder, S. 345, abrufbar a.a.O. (Fn. 43).

590 Ebd. Fn. 587; Kutscher, Der digitale Nachlass, 2015, S. 145.

591 Bräutigam in: Burandt/Rojahn (Hrsg.), nach $\$ 1922$ BGB Rn. 22; Bock, AcP (217) 2017, 370, 407 f.; Mayen in: DAV-Stellungnahme Nr. 34/2013, S. 75 ff.

592 Bock, AcP (217) 2017, 370, 408 ff.; Klas/Möhrke-Sobolewski, NJW 2015, 3473, 3477 f.; Solmecke/Köbrich/Schmitt, MMR 2015, 291, 292; Brisch/Müller-ter Jung, CR 2013, 446, 450; nicht eindeutig Frank/Helms, Erbrecht, $\$ 1$ Rn.9a: „einschränkende Auslegung [von $\$ 88$ Abs. 3 TKG] erscheint durchaus denkbar.“

593 Vgl. Kuntz, jM 2016, 190, 191 f.; kritisch auch Bräutigam in: Burandt/Rojahn (Hrsg.), nach $\$ 1922$ BGB Rn. 23. 
den. ${ }^{594}$ Ein schonender Ausgleich werde aber kaum erreicht, wenn die Vertraulichkeitsinteressen der Kommunikationspartner de facto vollständig zurückgestellt werden. ${ }^{595}$

\section{bb) Andere Ansicht: Verstoß gegen $₫ 88$ Abs. 3 S. 1 TKG}

Anderer Ansicht nach verstößt der Provider gegen das Fernmeldegeheimnis und seine Pflicht aus $\$ 88$ Abs. 3 S. 1 TKG, wenn und soweit er dem Erben Zugang zum Account des Erblassers verschafft. ${ }^{596}$ Danach sei die Kritik, die an den vier soeben dargestellten Begründungsansätzen der herrschenden Meinung geübt wird, durchschlagend. ${ }^{597}$ Hier stehen zwei Aspekte im Vordergrund: Zum einen wird die Wirkung der zivilrechtlichen Sukzession(sfiktion) für den öffentlich-rechtlichen Kontext des Telekommunikationsrechts bestritten. ${ }^{598}$ Das Fernmeldegeheimnis beabsichtige den Schutz der tatsächlich-real an der Kommunikation beteiligten Personen, sodass ein Wechsel in der Person eines Beteiligten - entgegen der erbrechtlichen Anordnung - nicht hinwegfingiert werden könne. ${ }^{599}$ Zum anderen wird auf das sogenannte kleine Zitiergebot aus $₫ 88$ Abs. 3 S. 3 TKG verwiesen. ${ }^{600}$ Danach müssen gesetzliche Ausnahmen von $₫ 88$ Abs. 3 S. 1 TKG das Fernmeldegeheimnis ausdrücklich in Bezug nehmen oder zumin-

594 Vgl. dazu etwa BVerfGE 134, 204, 223; BVerfGE 89, 214, 232 jew. m.w.N.; aus der Literatur zum Rechtsinstitut der praktischen Konkordanz vgl. nur LübbeWolff in: FS Kirchberg, S. 143 ff. m.w.N.

595 So Kuntz a.a.O. (Fn. 593); einen ausreichenden Schutz der Kommunikationspartner sehen aber Herzog/Pruns, Der digitale Nachlass in der Vorsorge- und Erbrechtspraxis, S. 97 die auf bestehende Löschungs-/Herausgabeansprüche gegen die Erben (freilich im Anschluss an deren Zugang zum Erblasseraccount) hinweisen.

596 Martini/Kienle, JZ 2019, 235, 235 f.; Mayen, AnwBl Online 2018, 466, 466 ff.; ders. in: DAV-Stellungnahme Nr.34/2013, S. 80 f.; Deusch, Digitales Sterben Das Erbe im Web 2.0, in: Taeger (Hrsg.), Tagungsband DSRI-Herbstakademie 2013, S. 437 f.; Kuntz, jM 2016, 190, 191 f.; Ubrenbacher, Digitales Testament und digitaler Nachlass, S. 181; KG Berlin, ZEV 2017, 386 Rn. 66 ff.

597 Siehe hierzu bereits die Nachweise in den Fn. 583, 586, 591 und 593.

598 Pointiert Mayen, AnwBl Online 2018, 466, 468 f.: Es handele sich „um eine Fiktion, die für die Zwecke des Erbrechts gilt, die aber auf das Fernmeldegeheimnis nicht übertragen werden kann." Ähnlich Martini/Kienle, JZ 2019, 235, 236.

599 Ebd. Fn. 598.

600 KG Berlin, ZEV 2017, 386 Rn. 81 f.; ferner Mayen in: DAV-Stellungnahme Nr. 34/2013, S. 81 f.; ders., AnwBl Online 2018, 466, 470. 
dest erkennbar eine gesetzgeberische Abwägung mit dem Fernmeldegeheimnis enthalten. ${ }^{601}$ Dem genüge $\ 1922$ Abs. 1 BGB aber gerade nicht. ${ }^{602}$

\section{cc) Rechtsdogmatische oder rechtspolitische Lösung}

Den für das Datenschutzrecht der DS-GVO festgestellten Vorteil der einzelfallorientierten Flexibilität weist das Telekommunikationsrecht gerade nicht auf. Es lässt nur die Extrempositionen eines umfassenden Zugangs der Erben oder ebendessen Ausschluss zu. Die Debatte im Spannungsfeld zwischen Rechtsnachfolge und Fernmeldegeheimnis wurde - vor allem bis zu dem sukzessionsfreundlichen Grundsatzurteil des $\mathrm{BGH}^{603}$ - auch deshalb so kontrovers geführt, weil damit die gesamte Handhabung des digitalen Nachlasses entscheidend beeinflusst wird. Ein vollständiger Ausschluss der Erben von allen Nutzerkonten des Erblassers wird im Übrigen lagerübergreifend als unbillig empfunden. Während die herrschende Ansicht dem mit teilweise ergebnisgetriebenen Auslegungsansätzen begegnet, ${ }^{604}$ fordern die anderslautenden Stimmen mehrheitlich Korrekturen durch den Gesetzgeber. ${ }^{605}$ Nicht zuletzt das Gutachten der Datenethikkommission sieht den Gesetzgeber auch nach dem Facebook-Urteil des BGH dazu aufgerufen, die Normkollision zwischen Fernmeldegeheimnis und Erbrecht aufzulösen. ${ }^{606} \mathrm{Da}$ den Interessen der Kommunikationspartner aber, wie gesehen, ${ }^{607}$ auch und gerade mit den Mitteln des Datenschut-

601 Bock in: Beck'scher TKG-Kommentar, $\$ 88$ TKG Rn. 28; Eckhardt in: Spindler/ Schuster (Hrsg.), Recht der elektronischen Medien, $\$ 88$ TKG Rn. 51.

602 KG Berlin, ZEV 2017, 386 Rn. 81 f.; ferner Mayen in: DAV-Stellungnahme Nr. 34/2013, S. 81 f.; ders., AnwBl Online 2018, 466, 470.

603 BGH NJW 2018, 3178 Rn. $56 \mathrm{ff}$.

604 Krit. Mayen, AnwBl Online 2018, 466, 471: eine „Logik, wonach nicht sein kann, was nicht sein darf, ist nun einmal keine überzeugende Auslegungsmethode."

605 Kuntz, jM 2016, 190, 191 f.; Mayen, AnwBl Online 2018, 466, 471; Bräutigam/ Herzog/Mayen/Redeker/Zuck in: DAV-Stellungnahme Nr. 34/2013, S. 92; Uhrenbacher, Digitales Testament und digitaler Nachlass, S. 223 f.; rechtspolitisch (wohl) etwas restriktiver Martini/Kienle, JZ 2019, 235, 240 f.; bemerkenswert insoweit KG Berlin, ZEV 2017, 386 Rn. 48: „[...] der Senat [hat] vollstes Verständnis für das Anliegen der Klägerin, [...] sieht sich aber rechtlich daran gehindert, diesem Ansinnen zum Erfolg verhelfen zu können."

606 Vgl. Gutachten der Datenethikkommission vom 23.10.2019, S. 111, abrufbar a.a.O. (Fn. 30).

607 Siehe dazu bereits S. $147 \mathrm{f}$. 
zes angemessen Rechnung getragen werden kann, ist der BGH nach hier vertretener Ansicht zurecht für seinen pragmatischen Umgang mit der gegenwärtigen Telekommunikationsrechtslage gelobt worden. ${ }^{608}$

\section{c) Fazit zu 2.}

Das Telekommunikationsrecht wird von (fast) ${ }^{609}$ allen Stimmen in der Debatte um den digitalen Nachlass als ein Hindernis auf dem Weg zu einer interessengerechten Lösung gesehen. Insofern hat der BGH mit seiner Auslegung des Begriffs der „anderen“ aus $\$ 88$ Abs. 3 S. 1 TKG einen lösungsorientierten Ausweg gefunden, ohne - wie von anderen Stimmen gefordert - ein Tätigwerden des Gesetzgebers abzuwarten. Ob damit aber eine dauerhafte Klärung der Debatte einhergegangen ist, darf bezweifelt werden. Der unionale Gesetzgeber prägt mit den aktuellen Vorhaben von EKEK $^{610}$ und ePrivacy-VO ${ }^{611}$ die Telekommunikationsrechtsordnung mit Wirkung auch für das nationale deutsche Telekommunikationsrecht. Hier werden die weiteren Entwicklungen genau zu beobachten sein, da das Ergebnis des BGH die Grenzen der Auslegung berührt und (auch insoweit) keinesfalls unumstritten ist. Wenn und soweit die Auslegung des Begriffs der Beteiligten an einem Kommunikationsvorgang und die damit verbundene Frage danach, wer als Dritter/Anderer zu gelten hat, (künftig) in den Anwendungsbereich des Europarechts fallen, ebnet das in einem derart umstritten Punkt den Weg zu einem ergebnisoffenen Vorabentscheidungsverfahren vor dem EuGH im Sinne von Art. 267 AUEV.

\section{Zwischenergebnis zu III.}

Während das materielle Erbrecht der richtige Ort ist, um über die persönlichkeitsrechtlichen Interessen des Erblassers zu entscheiden, werden die entsprechenden Belange der Kommunikationspartner primär durch das Datenschutz- sowie das Telekommunikationsrecht geschützt. Hierbei

608 In diesem Sinne insbesondere Hoeren, MMR 2018, 749, 749 f.; ferner Preu $\beta$, NJW 2018, 3146, 3146 f.; Härting/Dag, K\&R 2018, 638, 639.

609 Anders - soweit ersichtlich - nur Martini, JZ 2012, 1145, 1152 und (wohl) Martini/Kienle, JZ 2019, 235, $235 \mathrm{f}$.

610 Siehe hierzu S. $149 \mathrm{ff}$.

611 Dazu Martini/Kienle, JZ 2019, 235, 240 f.; Lieder/Berneith, ZRP 2020, 87, 89. 
haben sich de lege lata die datenschutzrechtlichen Vorgaben der DS-GVO als vorrangig geeignet erwiesen, um die mitunter widerstreitenden Interessen der Erben und der Kommunikationspartner einzelfallorientiert zu einem schonenden Ausgleich zu bringen. Danach stellt der umfassende Zugang der Erben zum Account des Erblassers die Regel dar, von der nach Maßgabe der DS-GVO im Einzelfall begründete Ausnahmen zu machen sind.

\section{Gestaltungsspielraum der Vertragsparteien}

Im nächsten Schritt ist zu prüfen, ob und inwieweit die Parteien des Nutzungsvertrags von den bislang ermittelten Ergebnissen durch Vereinbarung abweichen können. In der privatautonomen Rechtsordnung des BGB ist es den Parteien eines Vertrags grundsätzlich unbenommen, in Wahrnehmung ihrer Vertragsfreiheit etwa den Übergang vertraglicher Rechte und Pflichten im Erbfall zu beschränken oder auszuschließen. ${ }^{612}$ Treffen die Parteien des Nutzungsvertrags entsprechende Sonderregelungen im Wege einer Individualabrede (dazu 1.), sind regelmäßig (nur) die weiten Grenzen der $\mathbb{S} \mathbb{S} 138,242,313$ BGB zu beachten. ${ }^{613}$ Entsprechende AGBKlauseln (dazu 2.) obliegen zusätzlich der Einbeziehungs- und Inhaltskontrolle gem. $\$ \$ 305 \mathrm{ff}$. BGB. ${ }^{614}$ In jedem Fall ist die multipolare Rechtsbeziehung im digitalen Nachlass angemessen zu berücksichtigen - namentlich in Form der (datenschutzrechtlichen) Interessen der Kommunikationspartner, über welche die Vertragsparteien regelmäßig nicht allein disponieren können.

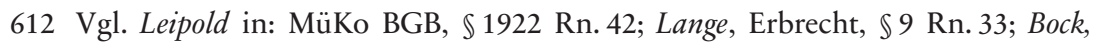
AcP (217) 2017, 370, 372; Kunz in: Staudinger, $\mathbb{1 9 2 2}$ BGB Rn. 166 ff., 636; BGH NJW 2018, 3178 Rn.24. Das gilt freilich nicht unbeschränkt, vgl. etwa $\$ 563$ BGB. Noch weiter geht Wehleit, MMR 2018, 279, 280 der bezogen auf Nutzungsverträge sogar grundsätzlich einen (konkludent) auf den Tod des Nutzers auflösend bedingten Vertrag annimmt.

613 Zur Vertragsfreiheit und ihren Grenzen, vgl. Looschelders, Schuldrecht, Allge-

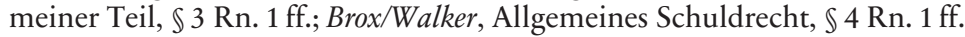

614 Die Bereichsausnahme für Verträge auf dem Gebiet des Erbrechts gem. $\$ 310$ Abs. 4 S. 1 BGB greift hier nicht ein, vgl. zu dessen Reichweite Becker in:

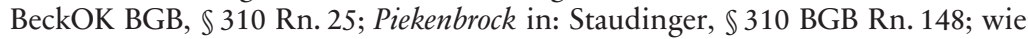
hier auch Thiesen, Daten in der Erbmasse, S. 56; eine (in Teilen obiter erfolgte) AGB-Prüfung im Bereich des digitalen Nachlasses findet sich bei BGH NJW 2018, 3178 Rn. 25 ff. 


\section{Regelungen im Wege der Individualabrede}

Die Untersuchung des individualvertraglichen Gestaltungsspielraums der Nutzungsvertragsparteien hat nur einen theoretischen Wert, soweit unter einer Individualabrede das Ergebnis einer gleichberechtigten, ergebnisoffenen Verhandlung verstanden wird. In der Praxis diktieren die Provider bestimmte Vertragsinhalte, welche die Nutzer lediglich im Ganzen annehmen oder ablehnen können. ${ }^{615}$ Zwar weisen einzelne Stimmen in der Literatur darauf hin, dass es dem Provider durchaus technisch möglich und auch rechtlich zulässig sei, beim Abschluss des Nutzungsvertrags mit Freitextfeldern in den AGB zu arbeiten. ${ }^{616}$ Dadurch könnte der Nutzer einen eigenen Vorschlag für das rechtliche Schicksal des Accounts und der darin enthaltenen Daten formulieren. In der Folge wäre entweder schon das Vorliegen von AGB zu verneinen, weil ein „Aushandeln“ im Sinne von $\$ 305$ Abs. 1 S. 3 BGB vorliegt, oder zumindest eine nach $\$ 305$ b BGB vorrangige „Individuelle Vertragsabrede“ gegeben. ${ }^{617}$ Realistischerweise müsste die Nachfolgeregelung aber eine besondere Priorität für den Anbieter haben, um aus dessen Sicht den zusätzlichen Aufwand zu rechtfertigen, der durch die Prüfung der jeweiligen nutzerseitigen Eingabe entsteht. ${ }^{618}$

615 Pointiert in diesem Sinne Bräutigam, MMR 2012, 635, 640: „Individuelle Aushandlungslösungen verbieten sich gegenüber Anbietern, die Millionen, ja Hunderte von Millionen Nutzer haben. [...] den Nutzern [bleibt] nichts anderes übrig, als die Bedingungen auf einer ,take it or leave it-Basis' anzunehmen." In diese Richtung auch Kunz in: Staudinger, $\mathbb{\$} 1922$ BGB Rn. 636 sowie die Studie des Fraunhofer Instituts für Sichere Informationstechnologie, S. 116, abrufbar a.a.O. (Fn. 7); hinzukommt, dass nach Ansicht von Dix, ZEuP 2017, 1, 4 wegen des „sozialen Anschluss- und Nutzungszwangs“ bei großen Internet-Plattformen selbst die zivilrechtliche Vorstellung der Vertragsabschlussfreiheit „zunehmend lebensfremd" ist.

616 Bock, AcP (217) 2017, 370, 411 f. dort in Fn. 294 sowie dies. in: Groll/Steiner (Hrsg.), Praxishandbuch Erbrechtsberatung, Rn. 20.119; Lieder/Berneith, FamRZ 2018, 1486, 1487; i.E. ähnlich auch Thiesen, Daten in der Erbmasse, S. 37 f., 70 f., der die Möglichkeit sieht, dem Nutzer ein einseitiges Bestimmungsrecht einzuräumen; zur a.A., wonach die Nutzer keine realistische Möglichkeit haben, individuelle abweichende Regelungen zur Vererbbarkeit zu treffen, vgl. Kunz in: Staudinger, $\mathbb{\$} 1922$ BGB Rn. 636 sowie Fußeder, Soziale Netzwerke im Nachlass, S. 221.

617 Zum Verhältnis von $₫ 305$ Abs. 1 S. 3 BGB und $₫ 305 b$ BGB vgl. Mäsch in: Staudinger, $\mathbb{} 305$ b BGB Rn. 2; Schmidt in: BeckOK BGB, $\mathbb{} 305$ b Rn. 8.

618 Thiesen, Daten in der Erbmasse, S. 9 beschreibt das Vorgehen der Provider als von Pragmatismus und Kosteneffizienz gekennzeichnet; zurecht skeptisch Kutscher, Der digitale Nachlass, S. $151 \mathrm{f}$. 
Denn der Provider muss jeweils sichergehen, dass er durch die Freitexteingabe des Nutzers nicht zu etwas verpflichtet wird, das entweder aus Rechtsgründen gar nicht oder aus wirtschaftlichen Gründen nur mit unverhältnismäßigem Aufwand erfüllt werden kann. ${ }^{619}$ Das (ökonomische) Interesse des Providers an der Vermeidung „toter“ Nutzerkonten ${ }^{620}$ stellt insofern keinen hinreichenden Antrieb dar, weil eine entsprechende Regelung der (Un-)Vererbbarkeit im Wege von AGB effizienter ist. ${ }^{621}$ Auch ein wahrnehmbares Drängen der Nutzer auf eine solche Selbstbestimmung durch Freitextfelder ist bislang nicht zu verzeichnen.

Weniger aufwändig in der Handhabung und daher aus Sicht des Providers möglicherweise attraktiver ist es, dem Nutzer mittels Auswahlbuttons die Wahl zwischen unterschiedlichen vorformulierten Anordnungsvarianten einzuräumen. ${ }^{622}$ Indes führt eine solche Lösung gerade nicht aus der AGB-Kontrolle heraus. ${ }^{623}$ Denn um AGB handelt es sich auch dann, wenn einer Vertragspartei nur die Wahl zwischen bestimmten, vom Verwender vorgegebenen Alternativen hat, weil bereits darin eine einseitige Ausnutzung der Vertragsgestaltungsfreiheit zu sehen ist. ${ }^{624}$ Solange der Nutzer keinerlei Möglichkeit hat, eigene Textvorschläge mit der effektiven Möglichkeit ihrer Durchsetzung in die Verhandlungen einzubringen, ist eine

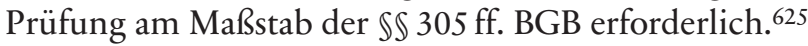

Nach alledem dürfte die praktische Relevanz der Individualabrede für den digitalen Nachlass von nur geringer Bedeutung sein. Den Schwerpunkt der hiesigen Untersuchung bildet daher der formularvertragliche Gestaltungsspielraum der Parteien.

619 Brisch/Müller-ter Jung, CR 2013, 446, 455; s. aber den Gestaltungsvorschlag nach Thiesen a.a.O. (Fn. 616), bei dem keine providerseitige Prüfung vorgesehen ist.

620 Damit argumentieren Lange/Holtwiesche, ZErb 2016, 125, 129; Budzikiewicz, AcP (218) 2018, 558, 590; ferner Bock, AcP (217) 2017, 370, 413.

$621 \mathrm{Zu}$ den rechtlichen Grenzen der Lösung mittels AGB siehe noch S. $164 \mathrm{ff}$.

622 Dafür plädieren daher auch Brisch/Müller-ter Jung a.a.O. (Fn. 619) sowie Litzenburger, FD-ErbR 2016, 375286.

623 Litzenburger, FD-ErbR 2018, 407688; Bock in: Groll/Steiner (Hrsg.), Praxishandbuch Erbrechtsberatung, Rn. 20.121; a.A. (wohl) Lieder/Berneith, FamRZ 2018, 1486, 1487 in Fn. 21.

624 BGH NJW-RR 2018, 693 Rn. 18; Schmidt in: BeckOK BGB, $\$ 305 b$ Rn.9 m.w.N.; Mäsch in: Staudinger, $\$ 305$ BGB Rn. 63 m.w.N.; Schuster in: Spindler/ Schuster (Hrsg.), Recht der elektronischen Medien, $\$ 305$ BGB Rn. $21 \mathrm{ff}$.

625 BGH NJW 2017, 2346 Rn. 9; Litzenburger, FD-ErbR 2018, 407688 m.w.N.; vgl. auch Mäsch in: Staudinger, $\mathbb{} 305$ BGB Rn. 63 a.E. 
2. Regelung im Wege Allgemeiner Geschäftsbedingungen

Regelmäßig stellt der Provider dem Nutzer bei Abschluss des Nutzungsvertrags für eine Vielzahl von Verträgen ${ }^{626}$ vorformulierte Vertragsbedingungen mit der Folge, dass der AGB-Begriff aus $₫ 305$ Abs. 1 S. 1 BGB erfüllt ist. ${ }^{627}$ Das gilt unabhängig von der Bezeichnung der Vertragsbedingungen durch den Provider zum Beispiel als „Nutzungsbedingungen“. 628 Sofern AGB vorliegen, ist in der weiteren Prüfung am Maßstab der $\mathbb{S}\{305 \mathrm{ff}$. BGB zwischen der Einbeziehungs- (dazu a) und der Inhaltskontrolle (dazu b) zu unterscheiden.

\section{a) Einbeziehungskontrolle}

Die AGB müssen wirksam in den Vertrag einbezogen werden, um für den Nutzungsvertrag Geltung zu beanspruchen. Nach Maßgabe von $\$ 305$ Abs. 2 Nr. 1 und 2 BGB werden die AGB nur dann Bestandteil des Vertrages, wenn der Provider bei Vertragsschluss ausdrücklich auf die AGB hinweist und dem Nutzer die Möglichkeit verschafft, in zumutbarer Weise von ihrem Inhalt Kenntnis zu nehmen. Ferner muss der Nutzer mit der Geltung der AGB einverstanden sein. Bezogen auf die wirksame Einbeziehung können die im Rahmen des E-Commerce entwickelten Grundsätze auf den Abschluss des Nutzungsvertrags übertragen werden. ${ }^{629}$ Hier hat sich in der Praxis durchgesetzt, dass der Vertragsschluss durch einen Klick technisch erst dann möglich ist, wenn der Kunde zuvor eine Box ange-

626 Für den hier praktisch relevanten Fall des Verbrauchervertrags kommt es gem. $\$ 310$ Abs. 3 Nr. 2 BGB auf das Merkmal der „Vielzahl von Verträgen“ nicht an.

627 Vgl. Berberich, MMR 2010, 736, 736 f.; auch Bräutigam, MMR 2012, 635, 637 und Jandt/Roßnagel, MMR 2011, 637, 639 setzen das Vorliegen von AGB voraus; einen Überblick über die Anbieterpraxis gibt die Studie des Fraunhofer Instituts für Sichere Informationstechnologie, S. 117 ff., abrufbar a.a.O. (Fn. 7).

628 Vgl. Berberich a.a.O. (Fn. 627) für weitere Beispiele, wie „Terms of Service“ oder „Rules of Conduct”, welche die Eigenschaft als AGB ebenfalls nicht berühren; s. auch Schuster in: Spindler/Schuster (Hrsg.), Recht der elektronischen Medien, $\$ 305$ BGB Rn. 12 und 43.

629 Vgl. dazu Föhlisch in: Hoeren/Sieber/Holznagel (Hrsg.), MMR-HdB, Teil 13 Rn. 115 ff. sowie Basedow in: MüKo BGB, $\$ 305$ Rn. 74 ff.; Schuster in: Spindler/ Schuster (Hrsg.), Recht der elektronischen Medien, $\$ 305$ BGB Rn. 32 ff.; s. aber auch Janal, NJW 2016, 3201, 3201 ff. zur AGB-Einbeziehungskontrolle bei Vertragsabschluss mittels Smartphones im sog. „M-Commerce“; dazu auch Basedow a.a.O. Rn. 76. 
klickt hat, in der auf die verlinkten AGB hingewiesen wurde. ${ }^{630}$ Bei sozialen Netzwerken ist der entscheidende Zeitpunkt des Vertragsschlusses im Sinne von $\ 305$ Abs. 2 BGB die Registrierung eines neuen Nutzers. ${ }^{631}$

Jedenfalls kein Vertragsbestandteil werden solche Bestimmungen, auf die zum Zeitpunkt des Vertragsschlusses überhaupt nicht hingewiesen wird. ${ }^{632}$ Das betrifft insbesondere Regelungen, die nicht Bestandteil der in Bezug genommenen Nutzungsbedingungen sind, sondern an anderer Stelle auf der Webseite des Providers mitgeteilt werden. ${ }^{633}$ So verhielt es sich mit der Richtlinie zum sogenannten Gedenkzustand von Facebook, die zum Zeitpunkt des BGH-Urteils statt in den AGB ausschließlich im HilfeBereich des sozialen Netzwerks ausgeführt wurde. ${ }^{634}$ Ein Nutzerkonto wird danach in den Gedenkzustand versetzt, sobald Facebook Kenntnis vom Tod des Kontoinhabers erlangt. Niemand - also auch kein Erbe kann sich bei einem in den Gedenkzustand versetzten Account anmelden und die Kontoinhalte einsehen. Ob darin ein formularvertraglicher Ausschluss der Vererbbarkeit des Facebook-Nutzerkontos zu sehen ist, musste der BGH mangels wirksamer Einbeziehung nicht entscheiden. ${ }^{635}$ Nunmehr wird der Gedenkzustand in Ziff. 4.5.5 der Nutzungsbedingungen zwar zumindest erwähnt. ${ }^{636}$ Es fehlt aber unverändert an einer weiteren Ausführung des Begriffs. ${ }^{637}$ Die praktischen und rechtlichen Konsequenzen aus einer Versetzung des Accounts in den Gedenkzustand erschließen sich dem Nutzer nach wie vor erst nach einer sorgfältigen Recherche im - nicht in Bezug genommenen - Hilfe-Bereich des Netzwerks.

630 Basedow a.a.O. (Fn. 629); Herzog/Pruns, Der digitale Nachlass in der Vorsorgeund Erbrechtspraxis, S. 106; Paul in: Hoeren/Sieber/Holznagel (Hrsg.), MMRHdB, Teil 7.4 Rn. 143 spricht von „click-wrap“.

631 Schuster in: Spindler/Schuster (Hrsg.), Recht der elektronischen Medien, $\$ 305$ BGB Rn. 44; Seidler, Digitaler Nachlass, S. 143.

632 Willems, ZfPW 2016, 494, 509; Herzog/Pruns, Der digitale Nachlass in der Vorsorge- und Erbrechtspraxis, S. 105 f.; Kutscher, Der digitale Nachlass, S. 122.

633 Ebd. Fn. 632.

634 BGH NJW 2018, 3178 Rn. 27; dazu auch Leipold in: MüKo BGB, $\mathbb{1} 1922$ Rn. 43. Zum maßgeblichen Beurteilungszeitpunkt vgl. Grüneberg in: Palandt, $\$ 307$ BGB Rn. 7 m.w.N.

635 Dessen ungeachtet findet sich ein entsprechendes obiter dictum, vgl. BGH NJW 2018, 3178 Rn. 28 ff. Siehe dazu noch S. $166 \mathrm{ff}$.

636 Abrufbar unter https:/www.facebook.com/legal/terms/update [17.11.2020].

637 Zum „Verstecken“von Regelungen in AGB (indes zu $₫ 307$ Abs. 1 S. 2 BGB) vgl. Bericht der Arbeitsgruppe „Digitaler Neustart“ der Konferenz der Justizministerinnen und Justizminister der Länder, S. 353, abrufbar a.a.O. (Fn. 43). 
Mithin werden die Regelungen zum Gedenkzustand noch immer nicht wirksam in den Vertrag einbezogen.

Den Schwerpunkt der hiesigen Darstellung stellt im Weiteren die Inhaltskontrolle dar, weil davon auszugehen ist, dass Provider ihre AGB so gestalten können, dass sie wirksam in den Vertrag einbezogen werden.

b) Inhaltskontrolle

Um den Nutzer als Vertragspartner des Verwenders vor einseitigen Benachteiligungen durch AGB zu schützen, unterliegen wirksam einbezogene Nutzungsbedingungen der Inhaltskontrolle gem. $\mathbb{S} 307$ bis 309 $\mathrm{BGB},{ }^{638}$ wenn und soweit dafür der Anwendungsbereich gem. $\$ 307$ Abs. 3 BGB eröffnet ist. ${ }^{639}$

Nach $₫ 307$ Abs. 3 S. 1 BGB unterliegen nur solche AGB der Inhaltskontrolle, durch die von Rechtsvorschriften abweichende oder diese ergänzende Regelungen vereinbart werden. Telos der Norm ist es, Bestimmungen über den Leistungsgegenstand und die Höhe der Gegenleistung - vorbehaltlich $₫ 307$ Abs. 3 S. 2 BGB - dem Kontrollregime zu entziehen, weil diese Fragen wegen ihrer besonderen Bedeutung ohnehin im Fokus der Vertragsparteien stehen und von den Beteiligten alleine zu regeln sind. ${ }^{640}$

Das Kammergericht Berlin sieht in AGB-Klauseln zur (Un-)Vererbbarkeit eines Accounts eine solche Leistungsbestimmung. ${ }^{641}$ Der Inhalt der Leistung werde lediglich beschrieben, indem der geschuldete Kontozugang als personenbezogen und auf die Lebzeiten des Nutzers begrenzt wer-

638 Vgl. Looschelders, Schuldrecht, Allgemeiner Teil, $\mathbb{1 6}$ Rn. 18; Brox/Walker, Allgemeines Schuldrecht, $\$ 4$ Rn. 33.

639 Zum Anwendungsbereich der AGB-Inhaltskontrolle s. Wurmnest in: MüKo BGB, $\mathbb{3} 307$ Rn. 1 ff.; von „Schranken der Inhaltskontrolle“ spricht Wendland in: Staudinger, $\mathbb{3} 307$ BGB Rn. 281 ff.; ausführlich zur Eröffnung der Inhaltskontrolle im Kontext des digitalen Nachlasses Thiesen, Daten in der Erbmasse, S. $56 \mathrm{ff}$.

640 Vgl. Wurmnest in: MüKo BGB, $\mathbb{} 307$ Rn. 13; Looschelders, Schuldrecht, Allgemeiner Teil, $\$ 16$ Rn. 18; Brox/Walker, Allgemeines Schuldrecht, $\$ 4$ Rn. 46; Schmidt in: BeckOK BGB, $\mathbb{3} 307$ Rn. 71 und 79; BGH NJW 2018, 534 Rn. 15 m.w.N. zur ständigen Rechtsprechung.

641 KG Berlin ZEV 2017, 386 Rn. 58 zum sogenannten Gedenkzustand bei Facebook. Die Argumentation des KG lässt sich indes auf ähnliche Klauseln betreffend die Unvererbbarkeit übertragen; zurecht kritisch dazu Gloser, DNotZ 2018, 306, 310; Herzog/Pruns, Der digitale Nachlass in der Vorsorge- und Erbrechtspraxis, S. 110; unklar Raude, ZEV 2017, 433, 437. 
de. ${ }^{642}$ Eine solche Lesart würde indes zu einer sehr starken Beschränkung des Anwendungsbereichs der Inhaltskontrolle führen. Die Leistungspflicht des Providers einschränkende oder ausgestaltende Bestimmungen sind aber gerade kontrollpflichtig. ${ }^{643}$ Für kontrollfreie Leistungsbeschreibungen im Sinne von $\mathbb{3} 307$ Abs. 3 S. 1 BGB verbleibt nach ständiger Rechtsprechung des BGH ,nur der enge Bereich von Regelungen, ohne deren Vorliegen mangels Bestimmtheit oder Bestimmbarkeit des wesentlichen Vertragsinhalts ein wirksamer Vertrag nicht mehr angenommen werden kann."“644 Die Frage nach der (Un-)Vererbbarkeit des Nutzungskontos stellt für den Nutzungsvertrag indessen kein solches essentiale negotii, sondern eine Modifikation des Leistungsumfangs des Providers (i.e. des Verwenders) dar. ${ }^{645}$ Insofern wird eine von dem disponiblen Grundsatz des $₫ 1922$ Abs. 1 BGB $^{646}$ abweichende und daher kontrollpflichtige Regelung getroffen. ${ }^{647}$

In der folgenden Untersuchung wird zwischen drei verschiedenen Klauseln unterschieden. In der Praxis der Provider gibt es erstens Klauseln, aus denen entweder explizit oder zumindest faktisch die Unvererbbarkeit des Accounts folgt (dazu aa), und zweitens Klauseln, durch die eine bestimmte Person unabhängig von einer etwaigen Erbenstellung mit der Wahrnehmung der Accountberechtigung betraut wird (dazu bb). Schließlich sind sogenannte Legitimationsklauseln in den Blick zu nehmen (dazu cc), in denen die Anforderungen an den Erbfolgenachweis definiert werden.

642 KG Berlin a.a.O. (Fn. 641).

643 BGH NJW 2018, 534 Rn. 15 m.w.N. zur ständigen Rechtsprechung; aus der Literatur vgl. etwa Schmidt in: BeckOK BGB, $\$ 307$ Rn. 80.

644 BGH NJW 2018, 534 Rn. 15; NJW 2008, 360 Rn. 18; ebenso bereits zur Vorgängervorschrift von $\$ 307$ Abs. 3 BGB (i.e. $\$ 8$ AGBG) BGH NJW 2001, 2635, 2636.

645 BGH NJW 2018, 3178 Rn. 29; sich dem BGH anschließend Bock in: Groll/Steiner (Hrsg.), Praxishandbuch Erbrechtsberatung, Rn. 20.127.

646 Zur Disponibilität der Vererbbarkeit vgl. bereits die Nachweise in Fn. 612.

647 Im Ergebnis wie hier auch der Bericht der Arbeitsgruppe „Digitaler Neustart“ der Konferenz der Justizministerinnen und Justizminister der Länder, S. 352, abrufbar a.a.O. (Fn. 43); Bock, AcP (217) 2017, 370, 412; Budzikiewicz, AcP (218) 2018, 558, 589; Herzog in: NK-Nachfolgerecht, Kap. 9 Rn.95; a.A. hingegen Thiesen, Daten in der Erbmasse, S. 35 ff., 61 f. sowie KG Berlin a.a.O. (Fn. 641). 


\section{aa) (Faktischer) Ausschluss der Vererbbarkeit}

Manche Anbieter schließen die Vererbbarkeit des Nutzungskontos in ihren AGB explizit aus. ${ }^{648}$ Bestimmungen anderer Anbieter, die zwar den erbrechtlichen Übergang selbst unberührt lassen, aber weitgehende Beschränkungen der Accountfunktionen ab dem Zeitpunkt des Erbfalls vorsehen, wirken sich für die Erben sehr ähnlich aus. Ein prominentes Beispiel dafür ist der sogenannte Gedenkzustand von Facebook. ${ }^{649}$ Ferner finden sich AGB-Klauseln, die entweder eine Beschränkung ${ }^{650}$ des Rechts auf Zugang zu oder die Löschung ${ }^{651}$ von inaktiven Nutzerkonten vorsehen. Als inaktiv gilt ein Account, sobald ein konkret bestimmter Zeitraum seit der letzten Anmeldung des Accountinhabers vergangen ist. ${ }^{652}$ Liegt der Grund für diese Inaktivität im Tod des Nutzers, ist aus Sicht der Erben - unabhängig von einer etwaigen Kenntnis des Providers vom Erbfall - wiederum eine mit expliziten Löschungsklauseln vergleichbare Wirkung zu konstatieren. Der BGH hat in seiner Facebook-Entscheidung grundsätzlich offengelassen, ob ein formularvertraglicher Ausschluss der Vererbbarkeit möglich ist. ${ }^{653}$

648 So z.B. bei Verizon Media-Accounts, zu denen der Webmail-Anbieter Yahoo gehört, vgl. Ziff. 3 lit. a) der AGB, abrufbar unter https://www.verizonmedia.com/ policies/ie/de/verizonmedia/terms/otos/index.html [17.11.2020]; ferner Apple bezüglich der Apple-ID für iCloud, vgl. Ziff. IV. D. der AGB, abrufbar unter https://www.apple.com/legal/internet-services/icloud/de/terms.html [17.11.2020].

649 S. die Bestimmungen zum Gedenkzustand bei Facebook, abrufbar a.a.O. (Fn. 636). Zur Wirkung des Gedenkzustands für den Account(inhaber) sowie zur - derzeit unwirksamen - Einbeziehungspraxis von Facebook vgl. bereits zuvor auf $S .162 \mathrm{f}$.

650 So z.B. der Webmail-Anbieter GMX, vgl. Ziff. 17.9 der AGB, abrufbar unter https://agb-server.gmx.net/gmxagb-de [17.11.2020].

651 So z.B. die Online-Dating-Plattform Parship, vgl. Ziff. 7.7 der AGB, abrufbar unter https://www.parship.de/termsandconditions/ [17.11.2020].

652 Das sind z.B. sechs Monate bei GMX (vgl. den Nachweis in Fn. 650) und 24 Monate bei Parship (vgl. den Nachweis in Fn. 651). Komplexer ist die Feststellung der Inaktivität bei GMail-Accounts, vgl. https://support.google.com/accounts/an swer/3036546?hl=de [17.11.2020].

653 BGH NJW 2018, 3178 Rn. 25; wie hier verstehen den BGH auch Lieder/Berneith, FamRZ 2018, 1486, 1487; Pruns, ErbR 2019, 456, 457; Goratsch, NZFam 2018, 810, 810; Traschler, ZEuP 2020, 168, 173; anders lesen das Urteil hingegen Litzenburger, FD-ErbR 2018, 407688 und Bräutigam in: Burandt/Rojahn (Hrsg.), nach $\$ 1922$ BGB Rn. 15. 
Bei der nachfolgenden Beurteilung der vorgenannten Klauseln sind zwei Aspekte zu beachten: Zum einen passen die Provider ihre Nutzungsbedingungen fortlaufend an die technischen und juristischen Entwicklungen an. ${ }^{654}$ Zum anderen unterscheiden sich die Formulierung und der Regelungsgehalt der AGB verschiedener Anbieter mitunter erheblich. ${ }^{65}$ Vor diesem Hintergrund sollen hier keine konkreten Klauseln geprüft, sondern verallgemeinerbare Parameter zur Bestimmung des Gestaltungsspielraums und seiner Grenzen entwickelt werden. Da keines der Klauselverbote aus $\mathbb{S} \$ 308,309$ BGB einschlägig ist, ${ }^{656}$ muss dafür die Generalklausel aus $\$ 307$ Abs. 1 und 2 BGB in den Blick genommen werden. ${ }^{657}$ Nach $₫ 307$ Abs. 1 S. 1 BGB sind „Bestimmungen in ABG [...] unwirksam, wenn sie den Vertragspartner des Verwenders entgegen den Geboten von Treu und Glauben unangemessen benachteiligen." Hierzu soll zuerst ein Maßstab anhand der gesetzlichen Regelbeispiele ${ }^{658}$ einer unangemessenen Benachteiligung aus $₫ 307$ Abs. 2 Nr. 1 BGB (dazu (1)) und $\$ 307$ Abs. 2 Nr. 2 BGB (da$\mathrm{zu}(2))$ gebildet werden, aus dem sich sodann Vorgaben für eine zulässige Klausel zur (Un-)Vererbbarkeit ableiten lassen (dazu (3)). Schließlich soll die sogenannte Frist-Variante gesondert untersucht werden (dazu (4)).

654 Bock, AcP (217) 2017, 370, 412 m.w.N.; bereits Deusch, ZEV 2014, 2, 7 empfiehlt Nutzern vor diesem Hintergrund, sich laufend über den aktuellen Standpunkt des/der Provider(s) zu informieren, zu denen ein Nutzungsverhältnis besteht.

655 Bock a.a.O. (Fn. 654); Herzog in: NK-Nachfolgerecht, Kap. 9 Rn. 90. Freilich sehen einige Anbieter überhaupt keine entsprechenden Klauseln in ihren AGB vor, vgl. etwa die AGB von LinkedIn, abrufbar unter https:/www.linkedin.com/ legal/user-agreement?trk=homepage-basic_join-form-user-agreement [17.11.2020], oder von XING, abrufbar unter https://www.xing.com/terms/xing [17.11.2020].

656 Biermann in: Münchener Anwaltshandbuch Erbrecht, $₫ 50$ Rn. 58; Bericht der Arbeitsgruppe „Digitaler Neustart“ der Konferenz der Justizministerinnen und Justizminister der Länder, S. 352, abrufbar a.a.O. (Fn. 43); Herzog/Pruns, Der digitale Nachlass in der Vorsorge- und Erbrechtspraxis, S. 110; offenlassend bezüglich $₫ 308$ Nr. 4 BGB der BGH NJW 2018, 3178 Rn. 32; dieses Ergebnis auch rechtspolitisch weiterhin wünschenswert finden Lieder/Berneith, ZRP 2020, 87, 88.

657 Zum Verhältnis von $₫ 307$ Abs. 1 und 2 zu $\$ \$ 308$ f. BGB vgl. Grüneberg in: Pa-

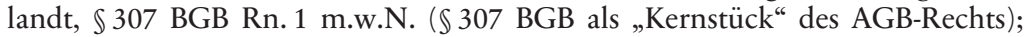
ferner Stadler in: Jauernig, BGB, $\$ 307$ Rn. 1.

658 Grüneberg in: Palandt, $₫ 307$ BGB Rn. 28: Die gesetzlichen Regelbeispiele des Abs. 2 sollen Abs. 1 inhaltlich konkretisieren; s. ferner Roloff/Looschelders in: Erman, $\$ 307$ BGB Rn. 1 . 
Nach $₫ 307$ Abs. 2 Nr. 1 BGB ist eine unangemessene Benachteiligung „im Zweifel anzunehmen, wenn eine Bestimmung mit wesentlichen Grundgedanken der gesetzlichen Regelung, von der abgewichen wird, nicht zu vereinbaren ist." Eine Klausel, die entweder explizit oder faktisch die Unvererbbarkeit des Vertragsverhältnisses zur Folge hat, weicht von der gesetzlichen Regelung des $₫ 1922$ Abs. 1 BGB ab. Denn danach ist eine Rechtsposition in der Regel und ohne anderslautende gesetzliche oder vertragliche Bestimmung vererblich. ${ }^{659}$ Aus dieser Feststellung allein kann indes noch nicht für jeden Fall einer Unvererbbarkeitsklausel auf das Vorliegen einer unangemessenen Benachteiligung geschlossen werden. ${ }^{660}$ Vielmehr muss genauer betrachtet werden, welche „wesentlichen Grundgedanken“ des \1922 Abs. 1 BGB durch $₫ 307$ Abs. 2 Nr. 1 BGB der Disposition mittels AGB entzogen sind. ${ }^{661}$ Die Vererbbarkeit einer Rechtsposition wird durch das Gesetz, das selbst zahlreiche Ausnahmen vom Grundsatz der Vererbbarkeit kennt, ${ }^{662}$ nämlich gerade nicht zwingend vorgegeben. ${ }^{663} \mathrm{Nicht}$ zuletzt ist die Disponibilität der Vererbbarkeit eines Vertrags im Rahmen von Individualvereinbarungen anerkannt. ${ }^{664}$ Aus $\$ 1922$ BGB folgt zwar der zwingende Charakter des Prinzips der Gesamtrechtsnachfolge; ${ }^{665}$ wel-

659 Bock, AcP (217) 2017, 370, 412 f.; dies. in: Groll/Steiner (Hrsg.), Praxishandbuch Erbrechtsberatung, Rn. 20.129 spricht anschaulich von Regel-Ausnahme-Verhältnis.

660 Sehr knapp daher Pruns, NWB 2014, 2175, 2185; Gloser, MittBayNot 2016, 12, $18 \mathrm{f} . ;$ auch Kutscher, Der digitale Nachlass, S. $126 \mathrm{f}$.

661 Vgl. Grüneberg in: Palandt, $\$ 307$ BGB Rn. 30 zur Unterscheidung zwischen gesetzlichen Zweckmäßigkeitserwägungen und formularmäßig nicht abbedingbaren Gerechtigkeitsgeboten; Roloff/Looschelders in: Erman, $\$ 307$ BGB Rn. 24; aus der Rechtsprechung siehe dazu z.B. BGH NJW 2007, 3637 Rn. 22.

662 Vgl. dazu bereits die Nachweise in Fn. 291.

663 Lange/Holtwiesche, ZErb 2016, 125, 128; Budzikiewicz, AcP (218) 2018, 558, 589; Bock, AcP (217) 2017, 370, 412 f.; dies. in: Groll/Steiner (Hrsg.), Praxishandbuch Erbrechtsberatung, Rn. 20.129; Kunz in: Staudinger, $\mathbb{} 1922$ BGB Rn. 636; Lieder/Berneith, FamRZ 2018, 1486, 1488.

664 Vgl. dazu bereits die Nachweise in Fn. 612.

665 Lange/Holtwiesche, ZErb 2016, 125, 128 mit Hinweis auf $\$ 2087$ BGB; MüllerChristmann in: BeckOK BGB, $\$ 1922$ Rn. 16; vertiefend zum Prinzip der Gesamtrechtsnachfolge Leipold in: MüKo BGB, $\mathbb{1} 1922 \mathrm{Rn} .180 \mathrm{ff}$. 
che Rechtsposition aber zu dem unaufteilbaren Nachlass gehört, ist eine der Universalsukzession vorgelagerte Frage. ${ }^{666}$

Diese vorgelagerte Frage darf der Einflusssphäre des Erblassers nicht vollständig entzogen werden. Sowohl $₫ 1922$ Abs. 1 BGB als auch die verfassungsrechtliche Erbrechtsgarantie des Art. 14 Abs. 1 S. 1 Var. 2 GG sehen vor, dass dem Erblasser eine Gestaltungs- und Verfügungsbefugnis über seine Rechte nicht nur lebzeitig, sondern ebenso von Todes wegen zukommt. ${ }^{667}$ Zwar muss im Falle vertraglicher Rechtspositionen - anders als bei dem Erblasser allein zustehenden absoluten Rechten - ein Ausgleich mit den Interessen des Verwenders als anderer Vertragspartei erfolgen. Allerdings wäre eine einseitige Bestimmung über die Vererbbarkeit durch den Verwender der AGB ohne eine Möglichkeit der Einflussnahme durch den Nutzer nicht mit der genannten Gestaltungs- und Verfügungsbefugnis des Erblassers vereinbar. ${ }^{668}$

Vor diesem Hintergrund stellt nicht die Vereitelung der Rechtsnachfolge durch die Erben den Leitbildverstoß im Sinne von $\$ 307$ Abs. 2 Nr. 1 BGB dar. ${ }^{669}$ Denn zum einen ist die schlichte Erberwartung im Zeitraum vor dem Erbfall bereits nicht schutzwürdig und zum anderen sind die Interessen der Vertragsparteien im Zeitpunkt des Vertragsschlusses (i.e. der Provider und der spätere Erblasser) richtigerweise maßgeblich. ${ }^{670}$ Vielmehr ist zu prüfen, ob die betreffende Klausel die Dispositionsbefugnis des

666 Lange/Holtwiesche a.a.O. (Fn. 665); Raude, ZEV 2017, 433, 437; in diese Richtung auch Budzikiewicz, AcP (218) 2018, 558, 589; Fußeder, Soziale Netzwerke im Nachlass, S. $226 \mathrm{f}$.

667 Leisner in: Isensee/Kirchhof (Hrsg.), Handbuch des Staatsrechts, Band VIII, $\$ 174$ Rn. 18; Große-Wilde in: Große-Wilde/Ouart (Hrsg.), Deutscher Erbrechtskommentar, Einleitung vor $\$ 1922$ BGB Rn. 1 mit Verweis auf BVerfGE 99, 341, 350 (Erbrecht diene der „Selbstbestimmung des Einzelnen im Rechtsleben“); im Kontext des digitalen Nachlasses dazu Bock, AcP (217) 2017, 370, 412 f.; dies. in: Groll/Steiner (Hrsg.), Praxishandbuch Erbrechtsberatung, Rn. 20.129; Lange/ Holtwiesche a.a.O. (Fn. 665); Raude, ZEV 2017, 433, 437.

668 Bock in: Groll/Steiner (Hrsg.), Praxishandbuch Erbrechtsberatung, Rn. 20.129; dies., AcP (217) 2017, 370, 412 f.; Lange/Holtwiesche a.a.O. (Fn. 665); Raude, ZEV 2017, 433, 437.

669 So (wohl) aber Kutscher, Der digitale Nachlass, S. $126 \mathrm{f}$., siehe indes ebd. S. 158 f.; Solmecke/Schmitt, ZD 2016, 186, 186; Seidler, Digitaler Nachlass, S. 144 f.; ähnlich Herzog in: NK-Nachfolgerecht, Kap. 9 Rn. 92; Alexander, notar 2018, 367, 367; nicht eindeutig BGH NJW 2018, 3178 Rn. 30.

670 Bock in: Groll/Steiner (Hrsg.), Praxishandbuch Erbrechtsberatung, Rn. 20.129; Lange/Holtwiesche, ZErb 2016, 125, 129. 
Erblassers so sehr einschränkt, dass darin eine unangemessene Benachteiligung liegt. ${ }^{671}$

(2) $₫ 307$ Abs. 2 Nr. 2 BGB

$\$ 307$ Abs. 2 Nr. 2 BGB enthält das zweite gesetzliche Regelbeispiel einer unangemessenen Benachteiligung, die danach widerleglich vermutet wird, ${ }^{672}$ "wenn eine Bestimmung wesentliche Rechte oder Pflichten, die sich aus der Natur des Vertrags ergeben, so einschränkt, dass die Erreichung des Vertragszwecks gefährdet ist." Der wichtigste Anwendungsfall von $₫ 307$ Abs. 2 Nr. 2 BGB ist die Freizeichnung von Kardinalpflichten. ${ }^{673}$ Es gehört zu den Hauptleistungspflichten des Providers, dem Nutzer Zugang zu dessen Account zu verschaffen. ${ }^{674}$ Das gilt unabhängig von der umstrittenen und von der jeweiligen Ausgestaltung des Internetdiensts abhängigen Frage nach der vertragstypologischen Einordnung des Nutzungsvertrags. ${ }^{675}$ Nach Ansicht des $\mathrm{BGH}^{676}$ und einiger Stimmen in der Literatur $^{677}$ wird diese Pflicht des Providers in einer den Vertragszweck gefährdenden Weise eingeschränkt, wenn der Provider unter Berufung auf eine

671 Bock, AcP (217) 2017, 370, 412 f.; dies. in: Groll/Steiner (Hrsg.), Praxishandbuch Erbrechtsberatung, Rn. 20.129; Lange/Holtwiesche a.a.O. (Fn. 665); Raude, ZEV 2017, 433, 437; wohl auch Biermann in: Münchener Anwaltshandbuch Erbrecht, $\mathbb{5} 50$ Rn. 60.

672 Zur Widerleglichkeit der Vermutung vgl. Grüneberg in: Palandt, $\$ 307$ BGB Rn. 28; kritisch dazu Wurmnest in: MüKo BGB, $\$ 307$ Rn. 65.

673 Grüneberg in: Palandt, $\mathbb{} 307$ BGB Rn. 33, 37; Berger in: Prütting/Wegen/Weinrich (Hrsg.), $\$ 307$ BGB Rn. 26; Schulte-Nölke in: HK-BGB, $\$ 307$ Rn. 17; Wurmnest in: MüKo BGB, $\$ 307$ Rn. 72.

674 So zurecht Bericht der Arbeitsgruppe „Digitaler Neustart“ der Konferenz der Justizministerinnen und Justizminister der Länder, S.359, abrufbar a.a.O. (Fn. 43); Budzikiewicz, AcP (218) 2018, 558, 589; BGH NJW 2018, 3178 Rn. 31.

675 Ebd. (Fn. 674); allgemein zur im Rahmen von $\$ 307$ BGB problematischen Vertragstypenbestimmung im Bereich elektronischer Medien, vgl. Schuster in: Spindler/Schuster (Hrsg.), Recht der elektronischen Medien, $\$ 307$ BGB Rn. 33 ff.; zur Vertragstypologie bei Nutzungsverträgen vgl. S. 33 ff.

676 BGH NJW 2018, 3178 Rn. 31 (obiter dictum zum Gedenkzustand bei Facebook).

677 Gloser, DNotZ 2016, 545, 548 f.; Budzikiewicz, AcP (218) 2018, 558, 589 f.; Alexander, notar 2018, 367, 367; wohl auch Herzog in: NK-Nachfolgerecht,

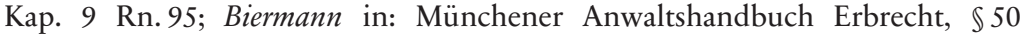
Rn. 62. 
Unvererbbarkeitsklausel dem Erben - ab dem Moment des Erbfalls als Nutzungsvertragspartei - den Accountzugang verwehrt.

Es stellt ein Problem an dieser Argumentation dar, dass sie sich unterschiedslos auf alle Internetdienste und auf jede Form einer Unvererbbarkeitsklausel übertragen lässt. Damit hätte der BGH aber die Tür zum formularmäßigen Ausschluss der Vererbbarkeit, die er explizit offengelassen hat, ${ }^{678}$ faktisch wieder verschlossen. Das kann in dieser Pauschalität nicht überzeugen. Telos der Inhaltskontrolle ist es, einen Ausgleich für die strukturell schwächere Verhandlungsposition des Nutzers (i.e. der Vertragspartner des Verwenders) zu schaffen. ${ }^{679}$ Insofern ist den obigen Ausführungen dann zu folgen, wenn der Provider unter Ausnutzung seiner überlegenen Stellung das eigene Pflichtenregime zulasten des Nutzers und seiner Erben verkürzt, ohne die Interessen seines Vertragspartners zu berücksichtigen. Wird dem Erblasser hingegen die Möglichkeit eingeräumt, etwa mittels Auswahlbuttons über das Schicksal seines Accounts im Erbfall (mit) zu bestimmen, dann sollte die Gestaltungsfreiheit der Parteien nicht unter Bezug auf $\$ 307$ Abs. 2 Nr. 2 BGB pauschal beschnitten werden. Denn der Erblasser kann im Einzelfall durchaus ein Interesse daran haben, dass seine Erben keinen Zugang zum Account erhalten. ${ }^{60}$ Kann er das in einer für den Provider bindenden Weise (z.B. bei Abschluss des Nutzungsvertrags) festlegen, wird die Gestaltungs- und Verfügungsbefugnis des Erblassers dadurch sogar erweitert. Denn so kann der Erblasser unterhalb der höheren Schwelle letztwilliger Verfügungen bereits auf vertraglicher Ebene disponieren. ${ }^{611}$ Dies mit Hinweis auf $\$ 307$ Abs. 2 Nr. 2 BGB zu unterbinden, hieße die Inhaltskontrolle (im Interesse der Erben) zulasten des Vertragspartners des Verwenders in Stellung zu bringen. Festzuhalten ist, dass auch im Rahmen von $\$ 307$ Abs. 2 Nr. 2 BGB kein Weg an einer einzelfallbezo-

678 BGH NJW 2018, 3178 Rn. 25; zu unterschiedlichen Lesarten des Urteils in diesem Punkt vgl. bereits die Nachweise in Fn. 653.

679 Vgl. bereits die Nachweise in Fn. 638.

680 Nachdrücklich in diesem Sinne Martini, JZ 2012, 1145, $1146 \mathrm{ff} . ;$ Rixecker in:

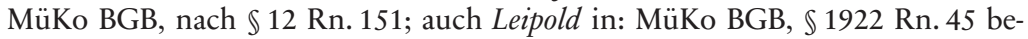
tont die Bewertung jeder Klausel im Einzelfall: Eine genauere Regelung durch AGB könne auch im Interesse des Account-Inhabers liegen.

681 Das dürfte vor allem für jüngere Nutzer attraktiv sein, denen Alexander, no$\operatorname{tar}$ 2018, 367, 367 eine häufige Verdrängung der Fragen des digitalen Nachlasses attestiert; a.A. Bericht der Arbeitsgruppe „Digitaler Neustart“ der Konferenz der Justizministerinnen und Justizminister der Länder, S. 365 f., abrufbar a.a.O. (Fn. 43), wonach die Gestaltungsmöglichkeiten des Erblassers mit einer Auflage ( $\mathbb{S} 1940,2192 \mathrm{ff} . \mathrm{BGB})$ ausreichen. 
genen Prüfung der Klausel vor dem Hintergrund der Details des Nutzungsverhältnisses vorbeiführen kann.

\section{(3) Vorgaben für eine zulässige Klausel zur (Un-)Vererbbarkeit}

Zuerst hat eine Klausel aus Gründen der Rechtssicherheit die Zuordnungsfunktion von $\$ 1922$ Abs. 1 BGB zu berücksichtigen. ${ }^{622}$ Unabhängig vom Schicksal des Accounts im Erbfall muss demnach eindeutig bestimmt sein, wer an den Accountinhalten berechtigt sein soll, solange und soweit diese nicht gelöscht werden. ${ }^{683}$

Im nächsten Schritt ist bezüglich der Parteiinteressen festzuhalten, dass die Annahme einer unangemessenen Benachteiligung im Sinne von $\$ 307$ Abs. 2 BGB eine Interessenabwägung voraussetzt, die sich im Falle von Nr. 1 am Merkmal der Unvereinbarkeit mit Grundgedanken des Gesetzes und im Falle von Nr. 2 an der Vertragszweckgefährdung entfaltet. ${ }^{684}$ Zwar kann eine solche Abwägung nur im Einzelfall bezogen auf eine konkrete Klausel und in Bezug auf einen bestimmten Internetdienst durchgeführt werden. ${ }^{685}$ Trotzdem lassen sich zumindest wiederkehrende Verwendungsinteressen der Provider identifizieren. Dazu gehören die rationalisierte Vertragsabwicklung durch die Vermeidung „toter“ Nutzerkonten. ${ }^{686} \mathrm{Ba}-$ siert der betreffende Internetdienst auf einem Data-for-Access-Finanzierungsmodell, ${ }^{687}$ wird der Provider ferner ein Interesse daran haben, „Zu-

682 BGH NJW 2018, 3178 Rn. 30; zustimmend Lieder/Berneith, FamRZ 2018, 1486, 1488; kein Risiko für klare Zuordnungsverhältnisse sieht hingegen Fußeder, Soziale Netzwerke im Nachlass, S. 226 f.; allgemein zur Zuordnungsfunktion des $\$ 1922$ Abs. 1 BGB vgl. Kunz in: Staudinger, $\$ 1922$ BGB Rn. 9 f.

683 Nach nicht näher begründeter Ansicht von BGH NJW 2018, 3178 Rn. 30 soll dem nicht genügen, wenn ausschließlich der Provider berechtigt ist. Dadurch entstehe ein "Datenfriedhof".

684 Ausführlich dazu Wendland in: Staudinger, $\$ 307$ BGB Rn. 253 ff. (zu Nr. 1) und Rn. 280 (zu Nr. 2) jeweils m.w.N.

685 Alexander, notar 2018, 367, 367 hingegen sieht in der restriktiven Linie des BGH NJW 2018, 3178 Rn. 28 ff. eine verallgemeinerbare Konstellation, die sich auf alle Arten von Anbietern internetbasierter Leistungen übertragen lasse.

686 Lieder/Berneith, FamRZ 2018, 1486, 1488; Lange/Holtwiesche, ZErb 2016, 125, 129; Budzikiewicz, AcP (218) 2018, 558, 590; ferner Bock, AcP (217) 2017, 370, 413; Herzog in: NK-Nachfolgerecht, Kap. 9 Rn. 97 („digitale Müll-Accounts“); a.A. Gomille, ZUM 2018, 660, 666, weder erhöhten sich die Geschäftschancen des Providers, noch verringere sich sein Aufwand durch die Stilllegung des Accounts.

687 Zur Funktionsweise mehrseitiger Finanzierung s. S. 37 ff. 
ordnungsverwirrung “688 zu vermeiden. Denn durch die Vermischung der Daten unterschiedlicher Nutzer (i.e. erst der Erblasser und dann die Erben) in einem Account wird die Präzision personalisierter Werbung und in der Folge das datenbasierte Geschäftsmodell beeinträchtigt. ${ }^{69}$ Das gilt zumindest, wenn neben dem lesenden Zugriff auch eine aktive Weiternutzung des Erblasseraccounts durch die Erben vorgesehen ist. ${ }^{690}$ Für den Nutzer ist vor allem das zuvor herausgearbeitete Interesse zu nennen, mittels einer ausreichenden Gestaltungs- und Verfügungsbefugnis als Erblasser über den Inhalt des eigenen Nachlasses bestimmen zu können.

Eine Klausel, die für den Todesfall des Nutzers alternativlos die Löschung seines Accounts mitsamt den darin gespeicherten Inhalten vorsieht, ${ }^{691}$ wird der nutzerseitigen Verfügungsbefugnis nicht genügen. ${ }^{692}$ Das gilt erst recht, wenn alle Accountinhalte ohne entsprechende Anweisung des Erblassers bei dem Provider verbleiben, ohne dass zumindest auch die Rechtsnachfolger darauf Zugriff haben. ${ }^{693}$ Der Anforderung ausreichender Verfügungsbefugnis wird hingegen eine Gestaltung gerecht, die dem Nutzer für den Fall seines Todes eine Wahlmöglichkeit darüber einräumt, wie mit seinem Account verfahren werden soll. ${ }^{694}$ Auer-Reinsdorff schlägt eine ausgewogene AGB-Gestaltung in diesem Sinne vor, die eine genaue Differenzierung auf Grundlage der Einstellungen des Accountinhabers ermög-

688 Gomille, ZUM 2018, 660, 666.

689 Gomille, ZUM 2018, 660, 666. Indes kann der Provider umgekehrt auch ein Interesse am fortgesetzten Betrieb eines populären, reichweitestarken Accounts haben.

690 Budzikiewicz, AcP (218) 2018, 558, 570 f. sieht hierin daher sogar ein Hindernis für die Vererbbarkeit des Rechts auf fortgesetzte Nutzung; ähnlich Leipold in: MüKo BGB, $\mathbb{S} 1922$ Rn. 37. Zur Reichweite des ererbten Rechts am Nutzerkonto vgl. bereits S. $100 \mathrm{ff}$.

691 So die Praxis bei Verizon Media- und iCloud-Accounts, vgl. a.a.O. (Fn. 648).

692 So auch Fußeder, Soziale Netzwerke im Nachlass, S. 235 ff.; Herzog/Pruns, Der digitale Nachlass in der Vorsorge- und Erbrechtspraxis, S. $111 \mathrm{f}$.

693 Mit dieser Begründung - nicht aber wegen Gefahren für die Rechtssicherheit ist dem Ergebnis in BGH NJW 2018, 3178 Rn. 30 zuzustimmen.

694 Entsprechend auch der Regulierungsansatz im Gutachten der Datenethikkommission vom 23.10.2019, S. 111, abrufbar a.a.O. (Fn. 30): „Die Datenethikkommission sieht die vorrangig anzustrebende Lösung in bewussten und informierten Dispositionen des Betroffenen noch zu Lebzeiten.“ Der Ansatz der DEK geht zurück auf Martini, JZ 2012, 1145, 1154; bekräftigt in Martini/Kienle, JZ 2019, 235, 241. 
licht. ${ }^{695}$ Einmal implementiert dürfte sich der dafür erforderliche Durchführungsaufwand für den Provider in einem zumutbaren Rahmen halten. Entscheidet der Inhaber sich in diesem Rahmen für die Löschung aller Accountinhalte, sind der Provider und die Erben allerdings auch daran gebunden. In diesem Fall kann nicht mit Hinweis auf das Interesse der Erben an einer zügigen Nachlassermittlung und -abwicklung für die Unzulässigkeit einer solchen Klausel gestritten werden. ${ }^{696}$ Denn entscheidend ist nicht der Accountzugang der Erben, sondern ob dem Erblasser die Entscheidung darüber, ob die Erben zugangsberechtigt sein sollen oder gerade nicht, einseitig vom Provider mittels AGB abgenommen wird. ${ }^{697}$

\section{(4) Frist-Variante}

Stellt man auf einem Spektrum dar, ob und inwieweit eine AGB-Gestaltung die Interessen des Erblassers berücksichtigt, dürften die alternativlose Löschung einerseits und das stark ausdifferenzierte Wahlrecht des Nutzers nach Auer-Reinsdorff ${ }^{698}$ andererseits als die beiden Extrempositionen gelten. Dazwischen liegt die Frist-Variante, bei welcher der Provider nach einer bestimmten Zeit der Inaktivität den Account löscht. ${ }^{699}$ Wenn auch nicht explizit, betrifft diese Konstellation bei praxisnaher Betrachtung je-

695 Auer-Reinsdorff in: Auer-Reinsdorff/Conrad (Hrsg.), Handbuch IT- und Datenschutzrecht, $\mathbb{2} 21 \mathrm{Rn}$. 84 f.: Danach kann der Nutzer zuerst wählen, ab wann sein Account als inaktiv gelten soll. Im Falle von Inaktivität nimmt der Provider Kontakt per E-Mail auf. Reagiert der Nutzer darauf wiederholt nicht, befolgt der Provider eine vorab bei Vertragsschluss vom Nutzer ausgewählte Anweisung (Löschung der Accountinhalte bzw. Zugangsgewährung gegenüber den Erben oder einer konkret bestimmten Vertrauensperson). Schließlich kann der Nutzer im Voraus bestimmte Inhalte über Filter als vertraulich markieren, welche den Erben/der Vertrauensperson vorenthalten bleiben; in diese Richtung auch der Regelungsvorschlag der FDP-Bundestagsfraktion, vgl. BT-Drs. 19/14044, S. 6 Ziff. 4.

696 Fußeder, Soziale Netzwerke im Nachlass, S. $234 \mathrm{ff}$.

697 Fußeder a.a.O. (Fn. 696); Lange/Holtwiesche, ZErb 2016, 125, 128 f.; Bock, AcP (217) 2017, 370, 412 f.; dies. in: Groll/Steiner (Hrsg.), Praxishandbuch Erbrechtsberatung, Rn. 20.129; auch Leipold in: MüKo BGB, $\mathbb{S} 1922$ Rn. 45 betont die Bewertung jeder Klausel im Einzelfall: Eine genauere Regelung durch AGB könne auch im Interesse des Account-Inhabers liegen.

698 S. dazu Fn. 695.

699 So die Praxis bei GMX und Parship, vgl. a.a.O. (Fn. 650 und 651). 
denfalls faktisch ${ }^{700}$ auch Erbfälle und dürfte im Falle einer ausreichend bemessenen Frist zur Wahrung der Interessen des Providers zulässig sein. ${ }^{701}$ Einige Stimmen in der Literatur fordern, dass die Klausel wegen des Informationsinteresses der Erben nur dann wirksam sein könne, wenn der Fristablauf bis zur Information der Erben über die formularvertragliche Vereinbarung gehemmt bleibe. ${ }^{702}$ Diese Anforderung dürfte für den Provider indes kaum zu erfüllen sein. Denn erstens hat der Anbieter nicht unbedingt positive Kenntnis davon, dass die Inaktivität auf dem Tod des Nutzers basiert. Zweitens kann der Provider beispielsweise bei Accounts, die der Erblasser ohne Kenntnis seiner Erben oder anderer Dritter unter einem Pseudonym eröffnet hat, nicht zur Ermittlung der Erben verpflichtet werden.

\section{(5) Zwischenergebnis zu aa)}

Die Vererbbarkeit eines Nutzerkontos kann in den AGB des Diensteanbieters nur ausgeschlossen werden, wenn der Gestaltungs- und Dispositionsbefugnis des Erblassers über den eigenen Nachlass ausreichend Rechnung getragen wird. Dem genügt eine Gestaltung, in welcher der Erblasser mittels Auswahlbuttons über den Übergang des Accounts beziehungsweise dessen Ausschluss entscheiden kann. Eine einseitig durch den Provider vorgegebene Bestimmung, wonach der Account nicht übertragbar ist, stellt hingegen eine unangemessene Benachteiligung des Nutzers im Sinne von $\$ 307$ Abs. 2 Nr. 1 BGB dar. Eine Klausel, welche die Löschung des Accounts nach einer Zeit der Inaktivität vorsieht, ist wirksam, wenn die vorgesehene Frist nicht zu kurz bemessen ist.

700 Kutscher, Der digitale Nachlass, S. 153 f. („Der Tod des Nutzers ist damit [...] erst recht erfasst.").

701 Leipold in: MüKo BGB, $\mathbb{1} 1922$ Rn. 45; Bock, AcP (217) 2017, 370, 413; Herzog in: NK-Nachfolgerecht, Kap. 9 Rn. 97; Seidler, NZFam 2020, 141, 144; im Grundsatz so auch Lieder/Berneith, FamRZ 2018, 1486, 1488.

702 So Lieder in: Erman, $\mathbb{\$} 1922$ BGB Rn. 14a und ders./Berneith, FamRZ 2018, 1486, 1488 die als Orientierungsgröße für die Frist auf $\$ 1944$ BGB verweisen. 


\section{bb) Bestimmung zur Accountberechtigung}

Die Anbieter Facebook ${ }^{703}$ und Google ${ }^{704}$ räumen dem Nutzer optional die Möglichkeit ein, eine Vertrauensperson zu benennen, welche im Todesfall die (alleinige) ${ }^{705}$ Berechtigung am Account erhalten soll. ${ }^{706}$ Diese Konstellation ist zunächst zivilrechtlich zu beurteilen (dazu (1)). Im Anschluss ist eine Bewertung unter datenschutzrechtlichen Gesichtspunkten vorzunehmen (dazu (2)).

\section{(1) Zivilrechtliche Beurteilung}

Der Nutzer kann eine beliebige Person als Vertrauensperson bestimmen. Handelt es sich dabei nicht um seine Erben, führt diese Vereinbarung zwischen Erblasser und Provider - ihre wirksame Einbeziehung vorausgesetzt $^{707}$ - zu einer Abweichung von dem gesetzlichen Ergebnis der Rechtsnachfolge des Erben in den Nutzungsvertrag. Abgewichen wird insoweit wiederum von dem in $\mathbb{1 9 2 2}$ Abs. 1 BGB enthaltenen Regel-AusnahmeVerhältnis, wonach Rechtspositionen regelmäßig auf die Erben übergehen. Indes handelt es sich bei dieser Abweichung nicht um eine unangemessene Benachteiligung im Sinne von $\$ 307$ Abs. 2 Nr. 1 BGB. ${ }^{708}$ Hier kann der zu Unvererbbarkeitsklauseln herausgearbeitete Maßstab herangezogen werden: Der Inhalt des Nachlasses ist eine primär vom Erblasser zu

703 Sogenannter Nachlasskontakt, vgl. Ziff. 4.5.5 der AGB, abrufbar a.a.O. (Fn. 636).

704 Sogenannter Kontoinaktivitätsmanager, vgl. https://support.google.com/accoun ts/answer/3036546?hl=de [17.11.2020].

705 Zur parallelen Berechtigung von Vertrauensperson und Erben, vgl. Thiesen, Daten in der Erbmasse, S. 72, 75 f.; Seidler, Digitaler Nachlass, S. 158 sieht die Befugnisse der Erben durch die Benennung einer Vertrauensperson nicht beschränkt.

706 In diese Richtung auch die vorgeschlagene Klausel von Auer-Reinsdorff in: Auer-

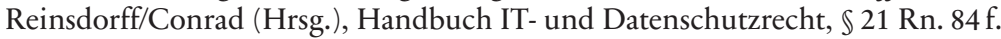

707 Das ist in der aktuellen Gestaltung zweifelhaft bei Google, da die Informationen zum Kontoinaktivitätsmanager lediglich im Hilfe-Bereich untergebracht sind, vgl. a.a.O. (Fn. 704). Zur Einbeziehungskontrolle vgl. bereits S. 162 f.; zur Abgrenzung von AGB zur Individualabrede bei Auswahlbuttons s. die Nachweise in Fn. $622 \mathrm{ff}$.

708 A.A. Gomille, ZUM 2018, 660, 666 der im „Nachlasskontakt“ von Facebook den unzulässigen Versuch einer rechtsgeschäftlichen Substitution der Universalsukzession durch eine Sondererbfolge sieht; kritisch auch Funk, Das Erbe im Netz, S. 35 f. („Lösung am Erbrecht vorbei“). 
bestimmende und dem Nachfolgemechanismus des $\$ 1922$ Abs. 1 BGB vorgelagerte Frage. Bei der Einsetzung einer Vertrauensperson wird die Rechtsposition der Accountinhaberschaft aber gerade im Wege des Vertrags zugunsten Dritter auf den Todesfall (gleichsam am Nachlass vorbei) ${ }^{709}$ auf die benannte Vertrauensperson übertragen. ${ }^{710}$ Es liefe dem Telos der Inhaltskontrolle zuwider, in diesem Fall zum Schutze der Erben von einer unwirksamen Abweichung von $\mathbb{1} 1922$ Abs. 1 BGB auszugehen, denn dadurch würde die Dispositions- und Verfügungsbefugnis des Erblassers beschränkt.

Das Dreiecksverhältnis ist folgendermaßen rechtsdogmatisch einzuordnen: Bei einem Nutzungsvertrag zwischen dem Provider als Versprechendem und dem Erblasser als Versprechensempfänger handelt es sich um das Deckungsverhältnis.711 Der Schenkungsvertrag zwischen dem Erblasser und der begünstigten Vertrauensperson ist das Valutaverhältnis. ${ }^{712}$ Dieser Schenkungsvertrag kommt (erst) nach dem Tod des Erblassers wirksam zustande, indem der Provider das Angebot des Erblassers zum Abschluss des Schenkungsvertrags an die Vertrauensperson weiterleitet, vgl. $\$ 130$ Abs. 2 BGB. Die Vertrauensperson kann das Angebot sodann annehmen, vgl. $\$ 153 \mathrm{BGB}$, ohne die Annahme gegenüber den Erben zu erklären, vgl. $\$ 151$ S. 1 BGB. ${ }^{713}$ Der Formmangel des Schenkungsvertrags im Sinne von $\$ 518$ Abs. 1 S. 1 BGB wird durch den Vollzug der Schenkung gem. $\$ 518$ Abs. 2 BGB geheilt. Der Anspruch der Vertrauensperson gegen den

709 Allgemein Janoschek in: BeckOK BGB, $\$ 331$ Rn. 5 (Erwerb des Leistungsanspruchs „unmittelbar unter Umgehung des Nachlasses.").

710 Herzog in: NK-Nachfolgerecht, Kap. 9 Rn. 81 hält einen einen Vertrag zugunsten Dritter auf den Todesfall oder eine postmortale Vollmacht für denkbar; Bock in: Groll/Steiner (Hrsg.), Praxishandbuch Erbrechtsberatung, Rn. 20.133 geht von einer „Außenvollmacht“ aus; nicht eindeutig Deusch, ZEV 2014, 2, 6; wie hier Thiesen, Daten in der Erbmasse, S. 71 ff.; Kutscher, Der digitale Nachlass, S. $152 \mathrm{ff}$.

711 Anders wohl Deusch, ZEV 2014, 2, 6 der lediglich ein Auftragsverhältnis zwischen Erblasser und Provider annimmt und auf $\$ 672$ S. 1 BGB hinweist; sich dem anschließend Seidler, Digitaler Nachlass, S. $157 \mathrm{f}$.

712 Thiesen, Daten in der Erbmasse, S. 73; Kutscher, Der digitale Nachlass, S. 154; a.A. Wunderlin/Bielajew, IPRB 2014, 223, 225: Benennung als Nachlassverwalter.

713 Die Erben könnten versuchen, das Angebot vor Zugang bei der Vertrauensperson zu widerrufen, vgl. $\$ 130$ Abs. 1 S. 2 BGB. Zu diesem als „Wettlauf zwischen Erben und Begünstigtem" bezeichneten Problem und seiner Vermeidung Gubitz, ZEV 2006, 333, 333 ff.; grundlegend Muscheler, WM 1994, 921, passim; zuerst zu der hier wichtigen Unterscheidung zwischen heimlicher und offenbarter Schenkung Hager in: FS von Caemmerer, S. 146 f.; dazu ferner Musielak in: MüKo BGB, $\mathbb{2} 2301$ Rn. 38 . 
Provider auf Zugang zum Erblasseraccount wird folglich mit dem Tod des Erblassers unmittelbar erworben im Sinne von $\$ 331$ BGB. ${ }^{714}$ Nach alledem scheitert die Benennung einer Vertrauensperson daher auch nicht an der für Verfügungen von Todes wegen (insbesondere gem. \$S 2064, 2231, 2247 BGB) vorgeschriebenen Form. ${ }^{715}$

\section{(2) Datenschutzrechtliche Bewertung}

So begrüßenswert die Möglichkeit zur Benennung einer Vertrauensperson im Sinne der zivilrechtlich vorgesehenen Gestaltungs- und Dispositionsbefugnis des Erblassers auch sein mag, ${ }^{716}$ muss der Blick doch auch auf die datenschutzrechtliche Umsetzbarkeit dieser Anordnung gelenkt werden. ${ }^{717}$ Denn je nach betroffenem Internetdienst werden der Vertrauensperson mit dem Zugang zum Erblasseraccount personenbezogene Daten von Kommunikationspartnern des Erblassers zugänglich gemacht. Das erfolgt dann zwar auf ausdrücklichen Wunsch des Erblassers. Dieser kann aber nicht über die datenschutzrechtlichen Betroffenenrechte seiner Kommunikationspartner disponieren. Dass der Erblasser vor seinem Tod faktisch ungehindert Inhalte seines Accounts mit derselben Vertrauensperson teilen kann, ändert daran nichts. Denn das Datenschutzrecht verpflichtet den Provider unmittelbar. Der Übertragung der Accountinhalte unter Vermittlung des Anbieters als Drittem wohnt eine spezifische datenschutzrechtliche Gefahr inne. ${ }^{718}$ Denn zum einen wird aus der Ausnahme (ein privater Nutzer gewährt einer anderen Person Zugang zum eigenen Account) die Regel (der Provider gewährt in jedem Erbfall einer Vertrauens-

714 Allgemein zur Konstruktion des Vertrags zugunsten Dritter auf den Todesfall, vgl. Musielak in: MüKo BGB, $\$ 2301$ Rn. $31 \mathrm{ff}$; Lange/Kuchinke, Erbrecht, S. 752 ff.; Große-Wilde in: Große-Wilde/Ouart (Hrsg.), Deutscher Erbrechtskommentar, $\$ 2301$ BGB Rn. $31 \mathrm{ff}$.

715 Herzog in: NK-Nachfolgerecht, Kap. 9 Rn. 81; kritisch dazu Raude, RNotZ 2017, 17, 23 wonach die „Aufweichung“ der Formvorschriften bedenklich erscheine.

716 Kutscher, Der digitale Nachlass, S. 154 („Die Provider könnten sich in dieser Hinsicht ein Beispiel am Vorreiter Google nehmen.“) und nochmals auf S. 161; kritischer sieht das Angebot hingegen Funk, Das Erbe im Netz, S. 35 f.

717 Dieser Aspekt fehlt bei dem Klauselvorschlag von Auer-Reinsdorff in: AuerReinsdorff/Conrad (Hrsg.), Handbuch IT- und Datenschutzrecht, $\$ 21$ Rn. $84 \mathrm{f}$.

718 Martini/Kienle, JZ 2019, 235, 235. 
person Zugang). ${ }^{719}$ Zum anderen hat der verarbeitende Provider andere technische Möglichkeiten als ein einzelner Nutzer, um effizient und umfassend in einer Vielzahl von Fällen Accountinhalte auf die jeweils benannte Vertrauensperson zu übertragen.

Vor der Zugangsgewährung gegenüber der Vertrauensperson muss sich der verarbeitende Provider deswegen mit denselben datenschutzrechtlichen Einwänden auseinandersetzen, wie er es bei der Zugangsgewährung gegenüber den Erben hätte tun müssen. ${ }^{720}$ Die veränderte Person des Datenempfängers ist dabei vor allem für die Abwägung von Bedeutung, die im Rahmen von Art. 6 Abs. 1 S. 1 lit.f) DS-GVO und - die Ausübung des Widerspruchs durch den Kommunikationspartner vorausgesetzt - erneut im Rahmen von Art. 21 Abs. 1 DS-GVO zu erfolgen hat. Denn während die Privatheitsinteressen der betroffenen Kommunikationspartner unverändert gewichtig sind, kann für das Verwendungsinteresse der Vertrauensperson beispielsweise nicht die Notwendigkeit einer zügigen Nachlassermittlung und -abwicklung angeführt werden. Die Vertrauensperson ist nicht demselben Haftungsrisiko aus $\$ 1967$ BGB ausgesetzt wie die Erben und hat auch nicht die knappe Ausschlagungsfrist des $\$ 1944$ BGB zu beachten.

Es kann keine abstrakte Aussage über die datenschutzrechtliche Zulässigkeit der Zugangsgewährung gegenüber der Vertrauensperson getroffen werden, weil die erforderliche Abwägung im Einzelfall erfolgen muss und ihr Ergebnis unter anderem von dem betroffenen Internetdienst abhängt. Wird als Vertrauensperson für ein geschäftlich genutztes E-Mail-Konto etwa der Mitgesellschafter eingesetzt, ${ }^{721}$ kann das sogar eher im Interesse der (dann wohl ebenfalls geschäftlichen) Kommunikationspartner sein, als es der Zugang durch die Erben wäre. Gleichwohl begegnet der verarbeitende Provider grundsätzlich höheren datenschutzrechtlichen Hürden bei der Zugangsverschaffung gegenüber der Vertrauensperson als gegenüber den Erben.

719 Die Argumentation von Martini/Kienle, JZ 2019, 235, 238 zum Zugang durch die Erben ist insoweit auf die Konstellation mit einer Vertrauensperson übertragbar.

720 Vgl. dazu ausführlich auf S. $118 \mathrm{ff}$.

721 Anschauliches Bild bei Podszun, GWR 2016, 37, 37 („twitternder Anwalt“). 


\section{(3) Zwischenergebnis zu bb)}

Bestimmt der Nutzer gegenüber dem Diensteanbieter eine Vertrauensperson, die im Erbfall die Zugangsrechte zum Account erhalten soll, ist darin ein Vertrag zugunsten Dritter auf den Todesfall zu sehen. Der Wirksamkeit dieser Vereinbarung stehen weder erbrechtlichen Formerfordernisse noch die Inhaltskontrolle aus $\$ \mathbb{S} 307 \mathrm{ff}$. BGB entgegen. Enthält der Account (auch) personenbezogene Daten der Kommunikationspartner des Erblassers, darf der Provider der Vertrauensperson indessen nur Zugang gewähren, wenn und soweit dies mit den datenschutzrechtlichen Vorgaben der DS-GVO in Einklang steht.

\section{cc) Legitimationsklauseln / Erbfolgenachweis}

Es kann irreversible Folgen haben, wenn der Provider einer unberechtigten Person Zugang zum Account des Erblassers verschafft. ${ }^{722}$ Denn sobald jemand Zugang zu dem Nutzerkonto hat, kann er die darin gespeicherten Inhalte vervielfältigen und exportieren. Zwar ist es möglich, dem Berechtigten im Nachhinein ebenfalls Zugang zu verschaffen. ${ }^{723}$ Da Daten aber nicht-rival, nicht-abnutzbar und nicht-exklusiv sind, ${ }^{724}$ ersetzt dies bezüglich des lesenden Zugriffs nicht den einmal gewährten Zugang des Unberechtigten, so wie es bei der Herausgabe einer dem Nachlass zugehörigen Sache im Sinne von $\$ 90$ BGB der Fall wäre, die zuvor einer unberechtigten Person ausgehändigt wurde. ${ }^{725}$ Anders verhält es sich nur mit der Möglichkeit zur fortgesetzten Nutzung des Accounts, die durch eine Zurücksetzung des Passworts dem Unberechtigten wieder vollständig entzogen werden kann. Vor dem Hintergrund des Bußgeld- und Haftungsrisikos für

722 Brisch/Müller-ter Jung, CR 2013, 446, 451.

723 Vorausgesetzt der Unberechtigte hat zuvor nicht irreversible Änderungen an den Accountinhalte veranlasst, vgl. Bericht der Arbeitsgruppe „Digitaler Neustart" der Konferenz der Justizministerinnen und Justizminister der Länder, S.358, abrufbar a.a.O. (Fn. 43) (providerseitige „Entgegennahme einer Löschungsanweisung").

724 Vgl. zu diesem Aspekt in der Diskussion um ein Recht an Daten Wagner in: MüKo BGB, $\sqrt{ } 823$ Rn. 335; Zech, Information als Schutzgegenstand, S. $117 \mathrm{ff}$., 328 f.; ders., CR 2015, 137, 140.

725 Vgl. Bericht der Arbeitsgruppe „Digitaler Neustart“, ebd. (Fn. 723), wonach im hiesigen Kontext „regelmäßig vollendete Tatsachen geschaffen werden.“ 
den Provider insbesondere unter Geltung der DS-GVO ${ }^{726}$ sind die Anbieter auf Rechtssicherheit angewiesen und haben daher ein Interesse daran, hohe Anforderungen an den Nachweis der Berechtigung an einem Account zu stellen. ${ }^{727}$ Dabei haben die Erben gleich zwei Nachweise zu führen: Die Berechtigung am Account ergibt sich kumulativ aus der Erbenstellung (dazu (1)) einerseits und der Nachlasszugehörigkeit des Accounts (dazu (2)) andererseits. ${ }^{728}$

\section{(1) Nachweis der Erbenstellung}

Eine für den Provider besonders sichere Variante des Nachweises über die Ebenstellung stellt wegen der Vermutungswirkung des $\$ 2365$ BGB ein Erbschein dar. ${ }^{729}$ In einer viel beachteten Entscheidung zur Legitimation gegenüber Sparkassen hat der BGH eine Klausel indessen für unwirksam erklärt, die bei kundenfeindlicher Auslegung die Vorlage eines Erbscheins zur Klärung der rechtsgeschäftlichen Berechtigung zwingend voraussetzte. ${ }^{730}$ Derart hohe Anforderungen gingen selbst über die grundbuchrechtlichen Anforderungen hinaus, vgl. $\$ 35$ Abs. 1 S. 2 GBO, ${ }^{731}$ und stellten

726 Dazu Frenzel in: Paal/Pauly (Hrsg.), Art. 83 DS-GVO Rn. 1; Faust/Spittkal Wybitul, ZD 2016, 120, $120 \mathrm{ff} . ;$ Boehm in: NK-Datenschutzrecht, Art. 83 DSGVO Rn. 3 vergleicht den Bußgeldrahmen mit dem des europäischen Kartellrechts; zu Kriterien und Praxis der Bußgeldzumessung vgl. Paal, RDV 2020, 57, $57 \mathrm{ff}$; neben das Bußgeld- tritt ein Haftungsrisiko auf Schadensersatz gem. Art. 82 DS-GVO, vgl. dazu Paal, MMR 2020, 14, 14 ff.; von „Verunsicherung“ der großen Anbieter berichtet Pruns, ErbR 2019, 456, 457, der ferner auf $\$ 206$ StGB hinweist; s. aber Deusch, ZEV 2018, 687, 689.

727 Solmecke/Köbrich/Schmitt, MMR 2015, 291, 294; Litzenburger, FD-ErbR 2018, 407688; s. aber ders. in: BeckOK BGB, $\mathbb{} 2232$ Rn. 25; a.A. hingegen Dopatka, NJW-aktuell 49/2010, S. 14 dem zufolge viele Anbieter in der Praxis „kulant“ seien und niedrige Anforderungen genügen ließen.

728 Vgl. Herzog in: NK-Nachfolgerecht, Kap. 9 Rn. 74 ff.; Brisch/Müller-ter Jung, CR 2013, 446, 451; Bock in: Groll/Steiner (Hrsg.), Praxishandbuch Erbrechtsberatung, Rn. $20.133 \mathrm{ff}$.

729 Stürner in: Jauernig, BGB, $\$ 2365$ Rn. 2: Die Vermutung für den Erbscheinserben ist im Prozess analog $\mathbb{\$} 292$ ZPO nur durch den Gegenteilsbeweis zu widerlegen.

730 BGH NJW 2013, 3716; dem zustimmend Keim, ZEV 2014, 277, 278; Fest, JZ 2014, 152, 156; Linardatos/Schwarz, EWiR 2013, 731, 732; instruktiv dazu Pieckenbrock/Rodi in: Staudinger, Anhang zu $\$ \$ 305-310$ BGB Rn. F $140 \mathrm{ff}$;; im Kontext des digitalen Nachlasses besonders Seidler, Digitaler Nachlass, S. $146 \mathrm{ff}$.

731 BGH NJW 2013, 3716 Rn. 38. 
eine unangemessene Benachteiligung der Erben dar, die ein Interesse „an einer möglichst raschen und kostengünstigen Abwicklung des Nachlasses“ haben. ${ }^{732}$ Mehrere Stimmen in der Literatur zum digitalen Nachlass fordern die Übertragung der bankenrechtlichen Rechtsprechung auf Legitimationsklauseln in Nutzungsverträgen. ${ }^{733}$

Allerdings ist fraglich, ob die Interessenlage bei der Legitimation gegenüber Banken und gegenüber den Anbietern von Internetdiensten vergleichbar ist. Denn über die eigenen Interessen hinaus hat der Provider anders als ein Kreditinstitut - in der mehrpoligen Situation des digitalen Nachlasses vielfach zusätzlich die Vertraulichkeitsinteressen der Kommunikationspartner zu wahren. Andererseits bleibt es nach der Rechtsprechung des BGH durchaus möglich, in begründeten Zweifelsfällen auf die Vorlage eines Erbscheins zu bestehen. ${ }^{734}$ Eine unangemessene Benachteiligung im Sinne von $₫ 307$ Abs. 2 Nr. 1 BGB stellt es lediglich dar, wenn die Vorlage eines Erbscheins ausnahmslos vorausgesetzt ist. ${ }^{735}$ Eingedenk dessen kann der Provider im Einzelfall entscheiden, ob und inwieweit er wegen Zweifeln an der Berechtigung eines (vermeintlichen) Erben zusätzliche Nachweise bis hin zum Erbschein fordert. ${ }^{736}$ Insofern wird (lediglich) die AGB-Gestaltungsmöglichkeit des Providers beschnitten, nicht aber ein grundsätzliches Risiko für die Interessen der Kommunikationspartner begründet. Daher ist der vorgenannten Meinung zuzustimmen, wonach die Rechtsprechung zur Legitimation gegenüber Banken herangezogen werden kann.

732 BGH NJW 2013, 3716 Rn. 35; bestätigt und vertieft in BGH NJW 2016, 2409 Rn. 20 mit zustimmender Anmerkung von Kroiß; zur Reaktion der Kautelarpraxis auf diese Rechtsprechung, vgl. Pieckenbrock/Rodi in: Staudinger, Anhang zu $\mathbb{S} \$ 305-310$ BGB Rn. F 140a.

733 Vgl. Leipold in: MüKo BGB, $\mathbb{1} 1922$ Rn. 45; Seidler, Digitaler Nachlass, S. 148; dies., NZFam 2020, 141, 144; Herzog in: NK-Nachfolgerecht, Kap. 9 Rn. 74; dies., NJW 2013, 3745, 3750; Solmecke/Köbrich/Schmitt, MMR 2015, 291, 294; Bericht der Arbeitsgruppe „Digitaler Neustart“ der Konferenz der Justizministerinnen und Justizminister der Länder, S. 355 ff., abrufbar a.a.O. (Fn. 43); Lieder/ Berneith, ZRP 2020, 87, 88; Lieder in: Erman, $\mathbb{1} 1922$ BGB Rn. 14a.

734 Pieckenbrock/Rodi in: Staudinger, Anhang zu $\$ \mathbb{S} 305-310$ BGB Rn. F 141 m.w.N.; Bock in: Groll/Steiner (Hrsg.), Praxishandbuch Erbrechtsberatung, Rn. 20.134.

735 BGH NJW 2013, 3716 Rn. 35; Linardatos/Schwarz, EWiR 2013, 731, 732.

736 Zwischen dem Haftungsrisiko auf Schadensersatz wegen unberechtigten Beharrens auf einem Erbschein, vgl. BGH NJW 2013, 3716 Rn. 41, und dem Bußgeldrisiko nach Art. 83 DS-GVO, vgl. die Nachweise in Fn. 726, wird sich der Provider im Zweifel für ersteres entscheiden. 
(2) Nachweis der Nachlasszugehörigkeit

Die Erbenstellung ist zwar eine notwendige, aber keine hinreichende Bedingung für die Rechtsnachfolge in den Nutzungsvertrag. Es muss sich nämlich zudem um den Account des Erblassers handeln. Der Nachweis der Nachlasszugehörigkeit stellt insbesondere bei solchen Nutzerkonten ein Problem dar, welche der Erblasser unter einem Pseudonym und ohne Angabe seiner persönlichen Kontaktdaten eröffnet hat. ${ }^{737}$ Das ist zum Beispiel bei Freemail-Accounts nicht ungewöhnlich. ${ }^{738}$ Die Eigentumsvermutung aus $\mathbb{1 0 0 6}$ BGB, die auch durch den Erbenbesitz des $₫ 857$ BGB begründet wird ${ }^{739}$ hilft mangels Sacheigenschaft des Accounts beziehungsweise des zugrundeliegenden Nutzungsverhältnisses grundsätzlich nicht weiter. ${ }^{740}$ Hier gelten die allgemeinen zivilprozessualen Regeln zur Darlegungs- und Beweislast, wenn und soweit die Erben von dem Provider zu einem bestimmten Account Zugang begehren. Soweit ersichtlich enthalten die AGB der Provider diesbezüglich keine speziellen Klauseln. In der Literatur findet sich der Ratschlag an den Erblasser, eine Liste mit seinen Nutzerkonten zu führen, sofern er seinen Erben nach dem Erbfall das Auffinden seiner Nutzerkonten sowie die Durchsetzung des übergegangenen Zugangsrechts erleichtern beziehungsweise ermöglichen möchte. ${ }^{71}$

737 Vgl. hierzu Brisch/Müller-ter Jung, CR 2013, 446, 451; ferner Herzog in: NK-Nachfolgerecht, Kap. 9 Rn. 75 ff.; Bock in: Groll/Steiner (Hrsg.), Praxishandbuch Erbrechtsberatung, Rn. 20.136.

738 Vgl. Brisch/Müller-ter Jung, CR 2013, 446, 451; Herzog in: NK-Nachfolgerecht, Kap. 9 Rn. 77.

739 Herrler in: Palandt, $₫ 857$ BGB Rn. 4; Berger in: Jauernig, BGB, $\mathbb{8} 857$ Rn. 3; Schäfer in: MüKo BGB, $\mathbb{} 857$ Rn. 9; BGH NJW 1993, 935, 937.

740 Bock in: Groll/Steiner (Hrsg.), Praxishandbuch Erbrechtsberatung, Rn. 20.136; anders nur für körperliche Speichermedien des Erblassers Herzog in: NK-Nachfolgerecht, Kap. 9 Rn. 75.

741 So z.B. Raude, RNotZ 2017, 17, 25 und Gloser, MittBayNot 2016, 101, 105 beide mit Hinweis auf etwaige Sicherheitsrisiken und Vorschlägen zu deren Minimierung; Steiner/Holzer, ZEV 2015, 262, 265 f.; Salomon, NotBZ 2016, 324, 328 f.; Herzog in: NK-Nachfolgerecht, Kap. 9 Rn. 77; ausführlich zur Vorsorge durch den Nutzer s. die Studie des Fraunhofer Instituts für Sichere Informationstechnologie, S. 175 ff., abrufbar a.a.O. (Fn. 7). 
dd) Zwischenergebnis zu b)

Einige Anbieter von Informations- und Kommunikationsdiensten sehen in ihren AGB Regelungen zum digitalen Nachlass vor. Die Klauseln unterscheiden sich stark und betreffen unterschiedliche Aspekte der Rechtsnachfolge in die nutzungsvertragliche Position des Erblassers. Grob lassen sich dabei drei unterschiedliche Themenkreise identifizieren. Die erste Gruppe von Klauseln betrifft die Frage, $o b$ überhaupt jemand in die vertragliche Position des Nutzers einrücken soll. Die zweite Art von Klauseln regelt, wer ein Zugriffsrecht erhalten soll. Drittens geht es darum, welche Anforderungen an den Nachweis der Berechtigung an einem Account gestellt werden können.

Die wirksame Einbeziehung der Klauseln vorausgesetzt, konzentriert sich die AGB-rechtliche Prüfung auf die Inhaltskontrolle und dort auf die Regelbeispiele einer unangemessenen Benachteiligung aus $₫ 307$ Abs. 2 Nr. 1 und 2 BGB. Hier ist vor allem das einfach- wie verfassungsrechtlich geschützte Recht des Erblassers zu berücksichtigen, selbst darüber disponieren zu können, welche seiner Rechtspositionen in seinen Nachlass fallen sollen. Nimmt der Provider dem Erblasser diese Gestaltungs- und Dispositionsbefugnis einseitig mittels AGB ab, ist darin eine unangemessene Benachteiligung im Sinne von $₫ 307$ Abs. 1 und 2 BGB zu sehen. Gleichzeitig findet die Freiheit des Erblassers ihre Grenze in den datenschutzrechtlichen Interessen seiner Kommunikationspartner. Der Provider ist als Adressat der DS-GVO zur Wahrung dieser Interessen verpflichtet und kann keine Anordnung des Erblassers erfüllen, die im Widerspruch zur DS-GVO steht. Hinsichtlich des Nachweises der Berechtigung darf der Provider in Zweifelsfällen auf die Vorlage eines Erbscheins bestehen. Im Übrigen muss er aber - entsprechend der Rechtsprechung zur Legitimation gegenüber Banken - auch andere Nachweise genügen lassen.

\section{Zwischenergebnis zu IV.}

In Wahrnehmung ihrer Privatautonomie können die Parteien des Nutzungsvertrags grundsätzlich von dem gesetzlich vorgesehenen Ergebnis zur Vererbbarkeit eines Nutzerkontos abweichen. Dieser vertragliche Gestaltungsspielraum hat indessen praktische Grenzen, die vor allem Individualabreden betreffen, und rechtliche Grenzen, welche sich primär bei der Verwendung von AGB zeigen. Im digitalen Massenverkehr stellen Individualabreden die seltene Ausnahme dar, weil für die Internetdiensteanbie- 
ter ein erheblicher Aufwand mit der Prüfung einzelner nutzerseitiger Eingaben verbunden ist. Die praktikablere Vorgehensweise, bei welcher der Provider seinem Vertragspartner lediglich einzelne vorformulierte Varianten anbietet, zwischen denen sich der Nutzer mittels Auswahl-Buttons entscheiden kann, ist bereits als Unterfall von AGB den $\$ \$ 305$ ff. BGB unterworfen. Bei der Wahrnehmung der vertraglichen Gestaltungsfreiheit durch solche Auswahlversionen oder im Wege herkömmlicher AGB hat der Provider die Interessen des Nutzers angemessen zu berücksichtigen und darf ihn nicht einseitig benachteiligen. Unabhängig von der gewählten Form der Vereinbarung führt für den Anbieter kein Weg aus seinen Pflichten nach Maßgabe der DS-GVO, die er gegenüber den Kommunikationspartnern des Erblassers trägt. Insbesondere können diese Pflichten nicht im Verhältnis zwischen Erblasser und Anbieter abbedungen werden.

\section{Zwischenergebnis zu B.}

Die Untersuchung des Accounts - respektive der Position des Erblassers im Nutzungsvertrag mit dem Anbieter von Informations- und Kommunikationsdiensten - als Erbrechtsobjekt verdeutlicht die Verflechtung unterschiedlicher Regelungsmaterien im Kontext des digitalen Nachlasses. Rein erbrechtlich ist zu konstatieren, dass die nutzungsvertragliche Rechtsposition des Erblassers grundsätzlich gem. $\$ 1922$ Abs. 1 BGB vererbt wird und dass die Erben als neue Accountinhaber gegenüber den Anbietern von Kommunikations- und Informationsdiensten dieselben Rechte und Pflichten tragen wie zuvor der Erblasser. Das schließt sowohl das Recht auf den lesenden Zugriff auf die gespeicherten Accountinhalte als auch das Recht zur aktiven Weiternutzung des Nutzerkontos ein. Das gilt jedenfalls, wenn und soweit die ursprünglichen Parteien des Nutzungsvertrags nicht in Wahrnehmung ihrer Vertragsfreiheit abweichende Regelungen vereinbart haben.

Jenseits des Erbrechts sind indes gesetzliche Pflichten zu beachten, die der Parteidisposition entzogen sind und welche die Ausübung der übergegangenen Rechte beschränken beziehungsweise ihrer Durchsetzung entgegenstehen können. Zum Beispiel dürfen die Erben ihr Recht zur aktiven Weiternutzung des Accounts nicht in unlauterer Weise ausüben - sie haben die Vorgaben das UWG zu beachten, soweit sie den Account gewerblich nutzen. Ferner kann auch und gerade der Provider seine vertraglichen Pflichten gegenüber den Erben nur in datenschutzrechtskonformer Weise erfüllen. Insbesondere ist er an die Vorgaben der DS-GVO gebunden. Da- 
mit wird der regelmäßig multipolaren Situation im sensiblen Kontext des Zugriffs auf Kommunikationsdaten Rechnung getragen.

Vor diesem Hintergrund kann bereits auf Grundlage des geltenden Rechts kein abstrakt-generelles Ergebnis zur Durchsetzbarkeit des Rechts auf lesenden Zugriff der Erben formuliert werden. Vielmehr müssen im konkreten Einzelfall die Funktionsweise des Internetdienstes, die Art der betroffenen Daten sowie die Interessen aller Beteiligten angemessen berücksichtigt werden. ${ }^{742}$ Erst recht müssen mit Blick auf die Zukunft die dynamischen Entwicklungen in technischer wie in rechtlicher Hinsicht laufend daraufhin überprüft werden, ob und inwieweit sie Einfluss auf die hier gefundenen Ergebnisse haben. Denn zum einen können sich technische Veränderung auf das Nutzungsverhalten und die darauf basierenden nutzerseitigen Erwartungen auswirken, denen etwa bei der erb- und datenschutzrechtlichen Beurteilung große Bedeutung zukommt. Zum anderen sind die Aktivitäten des (unionalen) Gesetzgebers insbesondere im Bereich des Telekommunikations- und Datenschutzrechts zu beobachten, in deren Anwendungsbereich die hier aufgeworfenen Fragen fallen (können).

\section{Urheberrechtlich geschützte Accountinhalte als Erbrechtsobjekt}

Neben den nutzungsvertraglichen Rechtspositionen des Erblassers sind auch seine Urheber- und Leistungsschutzrechte als Erbrechtsobjekte von Bedeutung für den digitalen Nachlass. Urheberrechts- oder zumindest Leistungsschutz nach Maßgabe des UrhG kann an unterschiedlichen Inhalten bestehen, die der Erblasser in seinen Account bei einem Internetdiensteanbieter einbringt. Beispielhaft seien hierfür die Veröffentlichung von Bildern auf Instagram, von Videos auf YouTube oder eines Blogbeitrags auf Tumblr genannt. ${ }^{73}$ Damit tritt neben die vertragliche Stellung als Accountinhaber gegebenenfalls eine zweite Rechtsposition im Verhältnis zu den Kontoinhalten - die des Inhabers der Urheber- bzw. Leistungs-

742 Nach hier vertretener Ansicht ist BGH NJW 2018, 3178 daher mehr Ausgangsals Endpunkt der Debatte zum Erbrecht an Nutzungsverträgen.

743 Mit weiteren Beispielen Bullinger in: Wandtke/Bullinger (Hrsg.), UrhG, $\mathbb{} 2$ Rn. 156 ff.; Bauer, User Generated Content, in: Große Ruse-Khan/Klass/v. Lewinski (Hrsg.), Nutzergenerierte Inhalte als Gegenstand des Privatrechts, S. 6 ff.; Reinemann/Remmertz, ZUM 2012, 216, 218 ff. 
schutzrechte an in den Account hochgeladenen Werken. ${ }^{744}$ Regelmäßig lässt sich der Provider von dem Nutzer eine Lizenz an diesen Immaterialgüterrechten einräumen, ohne die ein technisch reibungsloser Betrieb der Plattform nicht möglich wäre. ${ }^{745}$ Denn der Provider nimmt urheberrechtlich relevante Verwertungshandlungen (mindestens) im Sinne der $\$ \$ 16$ und 19a UrhG vor, indem er die nutzerseitig eingebrachten Inhalte auf seinem Server speichert und Dritten zugänglich macht. ${ }^{746}$ Zwar wirft diese Praxis bereits zu Lebzeiten des Nutzers rechtliche Fragen auf. ${ }^{747}$ Jedenfalls aber stehen sich im Nutzungsvertrag und im Lizenzverhältnis (noch) dieselben Parteien gegenüber. Das kann sich mit dem Tod des Nutzers ändern: Während das Urheberrecht regelmäßig gem. $\$ 28$ Abs. 1 UrhG in Verbindung mit $\$ 1922$ Abs. 1 BGB auf die Erben übergeht, ${ }^{748}$ kann die Rechtsnachfolge der Erben in den Nutzungsvertrag - wie gesehen - aus unterschiedlichen Gründen ausgeschlossen sein. Haben Erblasser und Provider beispielsweise den Übergang der Accountinhaberschaft wirksam vertraglich ausgeschlossen beziehungsweise anstelle der Erben eine Vertrauensperson als neuen Accountinhaber bestimmt, ${ }^{749}$ stehen dem Provider mit dem Urheberrechtsinhaber und Lizenzgeber einerseits und dem Accountinhaber andererseits unterschiedliche Personen gegenüber.

Im Folgenden sollen die Entstehung und die rechtlichen Konsequenzen dieses Auseinanderfallens näher beleuchtet werden. Dafür ist in einem ersten Schritt zu klären, ob und inwieweit Immaterialgüterrechte an den Accountinhalten bestehen und wie diese vererbt werden (dazu I.). Im Anschluss sind die an diesen Immaterialgüterrechten eingeräumten Lizenzen und deren Schicksal nach dem Tod des ursprünglichen Lizenzgebers genauer in den Blick zu nehmen (dazu II.). Zuletzt soll untersucht werden,

744 Sofern im Folgenden aus Gründen der besseren Lesbarkeit ausschließlich der Begriff des Urheberrechts genannt wird, sind Leistungsschutzrechte grundsätzlich mitgemeint, soweit im Text nicht ausdrücklich unterschieden wird.

745 Berberich, MMR 2010, 736, 737, 739; Paul in: Hoeren/Sieber/Holznagel (Hrsg.), MMR-HdB, Teil 7.4 Rn. 140; Solmecke ebd., Teil 21.1 Rn. 6; ders./Dam, MMR 2012, 71, 72.

746 Redeker in: Hoeren/Sieber/Holznagel (Hrsg.), MMR-HdB, Teil 12 Rn. 432; Berberich, MMR 2010, 736, 737; eingehend zu \19a UrhG Werner in: Ensthaler/ Weidert (Hrsg.), Urheberrecht und Internet, Kap. 3 Rn. 38 ff.; zweifelnd aber Imhof in: Bisges (Hrsg.), Handbuch Urheberrecht, Kap. 4 Rn. 139.

747 Vgl. v.a. zur Wirksamkeit der Lizenzeinräumung mittels AGB noch auf S. $198 \mathrm{ff}$.

748 Schack, Urheber- und Urhebervertragsrecht, Rn. 648 ff.; Hoche in: Wandtke/ Bullinger (Hrsg.), UrhG, $\mathbb{} 28$ Rn. 1; Rehbinder/Peukert, Urheberrecht, Rn. 988.

749 Siehe dazu S. 159 ff. 
ob sich aus dem Urheberrecht eine von der Stellung als Vertragspartei des Nutzungsvertrags unabhängige Berechtigung an den Accountinhalte ableiten lässt (dazu III.).

\section{Die Urheberrechte des Nutzers und ihre Vererbbarkeit}

Aus dem übergeordneten Themenkomplex „Urheberrecht und Erbrecht ${ }^{“ 750}$ sind hier diejenigen Aspekte herauszugreifen, die den Lebensbereich eines durchschnittlichen Nutzers sozialer Medien betreffen. Dabei sind zuerst die Accountinhalte in den Blick zu nehmen: Von den zahlreichen Medien, welche im Rahmen unterschiedlicher Internetdienste jeden Tag gespeichert und verbreitet werden, qualifizieren sich manche (aber nicht alle) für Urheber- oder zumindest für Leistungsschutz - wenn auch mitunter nur unter dem Gesichtspunkt der sogenannten „kleinen Mün$\mathrm{ze}^{\text {“ }} .{ }^{751} \mathrm{Ob}$ und inwieweit Immaterialgüterschutz besteht, kann zwar nur für einen konkreten Inhalt festgestellt werden. ${ }^{72}$ Grundsätzlich lassen sich aber durchaus Leitlinien anhand unterschiedlicher Werkarten und bezogen auf die Person des Schöpfers im Sinne von $\$ 7$ UrhG entwickeln (dazu 1.). Im Anschluss ist der in $\$ 28$ Abs. 1 UrhG vorgesehene Erbfolgemechanismus bezogen auf die vermögensrechtlichen und insbesondere die urheberpersönlichkeitsrechtlichen Befugnisse des Erblassers beziehungsweise der Erben darzustellen (dazu 2.).

750 Monografisch dazu Clément, Urheberrecht und Erbrecht, und Gloser, Die Rechtsnachfolge in das Urheberrecht, jeweils passim; für einen Überblick über die einschlägige Rechtsprechung vgl. Gergen, ZErb 2009, 42, 42 ff.

751 Kutscher, Der digitale Nachlass, S. 30 f.; zum Begriff vgl. Schack, Urheber- und Urhebervertragsrecht, Rn. 292 ff. („Als ,kleine Münze“ werden solche [Werke] bezeichnet, die nur ein soeben noch für ausreichend gehaltenes Minimum an Individualität aufweisen."); Bisges, GRUR 2015, 540, 540 ff.; ders., Die Kleine Münze im Urheberrecht, S. 18 ff.; Ensthaler in: Ensthaler/Weidert (Hrsg.), Urheberrecht und Internet, Kap. 2 Rn. $22 \mathrm{ff}$.

752 Schulze in: Dreier/Schulze (Hrsg.), UrhG, $\mathbb{} 2$ Rn. 36; eine dem Urheberrechtsschutz formal zugängliche Werkart allein reicht nicht aus, vielmehr muss im Einzelfall eine persönliche geistige Schöpfung im Sinne von $\$ 2$ Abs. 2 UrhG vorliegen, vgl. Ablberg in: BeckOK Urheberrecht, $\$ 2$ UrhG Rn. 50 ff.; Bullinger in: Wandtke/Bullinger (Hrsg.), UrhG, $\mathbb{} 2$ Rn. 2. 


\section{Urheber- und Leistungsschutzrechte des Nutzers}

Das UrhG unterscheidet in $\$ 2$ Abs. 1 UrhG zwischen unterschiedlichen Werkarten, denen die vom Nutzer in den Account eingebrachten Inhalte zugeordnet werden können. So kann ein Blogbeitrag als Sprachwerk im Sinne von $₫ 2$ Abs. 1 Nr. 1 UrhG, ein Foto als Lichtbildwerk im Sinne von $\$ 2$ Abs. 1 Nr. 5 UrhG und ein Video als Filmwerk im Sinne von $\ 2$ Abs. 1 Nr. 6 UrhG geschützt sein. Voraussetzung ist jeweils, dass in dem konkreten Werk eine persönliche geistige Schöpfung gem. $\$ 2$ Abs. 2 UrhG zu sehen ist. ${ }^{753}$ Es muss die individuelle Kreativität des Urhebers in dem Werk zum Ausdruck gebracht werden, indem ein bestehender Spielraum für kreative Entscheidungen ausgenutzt wird. ${ }^{754}$

\section{a) Unterscheidung der Kontoinhalte nach Werkarten}

Bei der urheberrechtlichen Beurteilung der großen Zahl unterschiedlicher Accountinhalte ist eine Orientierung anhand der verschiedenen Werkkategorien möglich. So ist für Sprachwerke festzuhalten, dass nicht jeder „Tweet“ auf Twitter oder jedes „Statusupdate“ bei Facebook eine persönliche geistige Schöpfung im Sinne von $\$ 2$ Abs. 2 UrhG darstellt. ${ }^{755}$ Gleichwohl reicht die Vielfalt nutzergenerierter Inhalte auch in dieser Werkart hin zu Beiträgen, die Ausdruck der Individualität ihres Schöpfers sind: ${ }^{756}$ Man denke etwa an ein für einen Podcast vorbereitetes Interview, an eine ausführliche Film- oder Buchrezension oder an ein per Privatnachricht

753 Schulze in: Dreier/Schulze (Hrsg.), UrhG, $\mathbb{} 2$ Rn. 6 („einheitliche Schutzvoraussetzung"); Ablberg in: BeckOK Urheberrecht, $\$ 2$ UrhG Rn. 50.

754 Loewenheim/Leistner in: Schricker/Loewenheim (Hrsg.), $\$ 2$ UrhG Rn. 30; eingehend zu den Anforderungen des $\$ 2$ Abs. 2 UrhG vgl. Schulze in: Dreier/Schulze (Hrsg.), UrhG, $\$ 2$ Rn.36ff.; Dreyer in: Dreyer/Kotthoff/Meckel/Hentsch (Hrsg.), Urheberrecht, $\$ 2$ UrhG Rn. 18 ff.

755 Wie hier Wiebe in: Spindler/Schuster (Hrsg.), Recht der elektronischen Medien, \$2 UrhG Rn.16f.; Budzikiewicz, AcP (218) 2018, 558, 567 f.; Bock, AcP (217) 2017, 370, 381; einen Schutz auch von Tweets können sich hingegen vorstellen Dreyer in: Dreyer/Kotthoff/Meckel/Hentsch (Hrsg.), Urheberrecht, $\$ 2$ UrhG Rn. 312 und Krieg, K\&R 2010, 73, 75; zur Maßstabsbildung im analogen Bereich instruktiv Schack, Urheber- und Urhebervertragsrecht, Rn. 204 f.

756 Mit Beispielen zur Abgrenzung Reinemann/Remmertz, ZUM 2012, 216, 218 f.; Bullinger in: Wandtke/Bullinger (Hrsg.), UrhG, $\mathbb{2}$ Rn. 159; Bauer, User Generated Content, in: Große Ruse-Khan/Klass/v. Lewinski (Hrsg.), Nutzergenerierte Inhalte als Gegenstand des Privatrechts, S. 6 f. 
übermitteltes Liebesgedicht ${ }^{757}$. Kein Schutz kommt hingegen schlichten Textnachrichten zu, die lediglich dem Austausch über den Alltag oder etwa zur Koordinierung einer Verabredung dienen.

Für Lichtbild- und Filmwerke ist darauf hinzuweisen, dass das UrhG unterhalb der Schwelle von $\$ 2$ Abs. 2 UrhG für Lichtbilder gem. $\$ 72$ UrhG und für Laufbilder gem. $\$ 95$ UrhG Leistungsschutz gewährt. ${ }^{758}$ Damit dürfte der Mehrheit der bei Instagram oder Pinterest eingestellten Fotografien und der bei YouTube und Vimeo hochgeladenen Videos zumindest Leistungsschutz zukommen. ${ }^{759}$ Auf den einschlägigen Plattformen werden aber nicht nur Amateurfilme oder private Urlaubsfotos geteilt, sondern auch aufwendig produzierte und nachbearbeitete Kurzfilme oder Portraitfotografien veröffentlicht, für welche Urheberschutz angenommen werden kann. Die ständige Praxis der Plattformbetreiber, sich im Rahmen des Nutzungsvertrags umfassende Nutzungsrechte an den Inhalten der Nutzer einräumen zu lassen, ist insofern jedenfalls als Hinweis auf die praktische Relevanz der Urheber- und Leistungsschutzrechte zu werten. ${ }^{760}$

Im Übrigen ist der Katalog des $₫ 2$ UrhG nicht abschließend, ${ }^{761}$ sodass gerade vor dem Hintergrund der sich ständig wandelnden Ausdrucksformen im Digitalen an ungenannte Werkarten im Sinne des UrhG zu denken ist. ${ }^{762}$ Zum Beispiel könnte ein Instagram Account selbst ein urheberrechtliches Werk darstellen. ${ }^{763}$ Zwar sind die Gestaltungsmöglichkeiten der Nutzer durch die Vorgaben der Plattform faktisch eingeschränkt, sodass die Werkqualität der meisten Nutzerkonten abzulehnen sein wird. Instagram Accounts kommen für Urheberrechtsschutz aber zumindest

757 Mit einem Beispiel dazu Krieg, K\&R 2010, 73, 75.

758 Kutscher, Der digitale Nachlass, S. 30; das Verhältnis von Filmwerk zu Laufbildern entspricht demjenigen von Lichtbildwerk zu Lichtbild, vgl. Seifert/Wirth in: HK-UrhG, $\mathbb{} 2$ Rn. 27.

759 So für Videos Wiebe in: Spindler/Schuster (Hrsg.), Recht der elektronischen Medien, $\mathbb{S} 95$ UrhG Rn. 3; Budzikiewicz, AcP (218) 2018, 558, 568; Katzenberger/N. Reber in: Schricker/Loewenheim (Hrsg.), $\$ 95$ UrhG Rn. 12; Kutscher, Der digitale Nachlass, S. 30; zu Lichtbildern Gomille, ZUM 2018, 660, $661 \mathrm{f}$.

760 Zur Lizenzierung und ihrer Wirksamkeit vgl. noch S. $194 \mathrm{ff}$.

761 Der Wortlaut („insbesondere“) ist insoweit eindeutig, vgl. Seifert/Wirth in: HKUrhG, $\mathbb{2}$ Rn. 2; Schulze in: Dreier/Schulze (Hrsg.), UrhG, $\mathbb{2}$ Rn. 3.

762 Podszun in: FS 50 Jahre UrhG, S. 361 ff.; Bullinger in: Wandtke/Bullinger (Hrsg.), UrhG, $\mathbb{2}$ Rn. 159 (das UrhG ist „offen für jegliche neue Ausdrucksformen.“).

763 Zurückhaltend, aber grundsätzlich offen Bullinger in: Wandtke/Bullinger (Hrsg.), UrhG, $\mathbb{} 2$ Rn. 159 f.; a.A. Kutscher, Der digitale Nachlass, S. 42 f.; ausführlich zur etwas anders gelagerten Frage nach dem Urheberschutz an Computerspiel-Accounts s. Gräber, Rechte an Accounts, S. 63 ff. 
eher in Betracht als Konten anderer Internetdienste, da hier das Gesamtkonzept eines Accounts über den einzelnen Beitrag hinaus mitunter sogar das primäre Gestaltungsobjekt sein kann. So werden Fotografien, die bei Instagram durch den Nutzer als einzelne Beiträge veröffentlicht werden, durch die Verantwortlichen mancher Accounts so ausgewählt beziehungsweise angefertigt, dass sie einem übergeordneten Konzept für den Account entsprechen. Dieses Konzept kann thematisch-inhaltlicher und/oder ästhetisch-gestalterischer Natur sein. Es ist nicht auszuschließen, dass manche Nutzerkonten als solche auf diese Weise zu einer persönlichen, geistigen Schöpfung gem. $\$ 2$ Abs. 2 UrhG werden.

\section{b) Der Nutzer als Schöpfer im Sinne von $\$ 7$ UrhG}

Nach $₫ 7$ UrhG ist Urheber(rechtsinhaber) der Schöpfer des Werkes. Im Falle von Lichtbildschutz wird gem. $\$ 72$ Abs. 2 UrhG der Lichtbildner, im Falle von Laufbildschutz gem. $\$ \$ 95,94$ UrhG der Laufbildhersteller der Leistungsschutzrechtsinhaber. ${ }^{764}$

Keinen Schutz erlangt der Nutzer demnach an solchen Accountinhalten, die er gar nicht selbst geschaffen und unter Umständen sogar unter Verletzung fremder Urheberrechte in seinen Account eingebracht hat. ${ }^{765}$ Das schließt im Account des Erblassers gespeicherte, aber von einem Kommunikationspartner erhaltene Inhalte wie empfangene Privatnachrichten aus. Anders liegt jedoch der Fall bei vom Nutzer unter Verwendung vorhandenen Materials selbst erzeugten sog. Mash-Ups, für die - unabhängig von der Rechtmäßigkeit der Nutzung des Materials - Urheberrechtsschutz in Betracht kommen kann. ${ }^{766}$

764 Vgl. zur Leistungsschutzrechtsinhaberschaft bei Laufbildern Katzenberger/N. Reber in: Schricker/Loewenheim (Hrsg.), $\$ 95$ UrhG Rn. 19 f. und bei Lichtbildern Schulze in: Dreier/Schulze (Hrsg.), UrhG, $\$ 72 \mathrm{Rn} .32 \mathrm{f}$.

765 Bauer, User Generated Content, in: Große Ruse-Khan/Klass/v. Lewinski (Hrsg.), Nutzergenerierte Inhalte als Gegenstand des Privatrechts, S. $15 \mathrm{ff}$. problematisiert das Themenfeld nutzergenerierter Inhalte primär vor diesem Hintergrund; zum sogenannten „Schöpferprinzip“, vgl. Hoeren in: Loewenheim (Hrsg.), Handbuch des Urheberrechts, $\mathbb{1} 10$ Rn. 1 ff.

766 Bullinger in: Wandtke/Bullinger (Hrsg.), UrhG, $\mathbb{2}$ Rn. 159; zur Differenzierung zwischen der Schutzfähigkeit einer Bearbeitung, $\mathbb{} 3$ S. 1 UrhG, und den Voraussetzungen für eine (rechtmäßige) Veröffentlichung/Verwertung der Bearbeitung, $\mathbb{} 23$ S. UrhG, siehe Schulze in: Dreier/Schulze (Hrsg.), UrhG, $\mathbb{} 23$ Rn. 2 m.w.N. 
c) Zwischenergebnis zu 1.

Auch wenn es eine Frage des Einzelfalls ist, ob und inwieweit Schutzrechte nach Maßgabe des UrhG an Accountinhalten bestehen, lassen sich nach alledem bestimmte Grundsätze festhalten: Keinesfalls genießt der gesamte Accountinhalt Urheberrechtsschutz. Vor allem enthaltene Chat-Verläufe, die lediglich dem gewöhnlichen Austausch unter Nutzern dienen, sind nicht geschützt. Für die vom Erblasser formulierten Nachrichten (und Statusmeldungen) scheitert ein Schutz regelmäßig an den Anforderungen aus \2 Abs. 2 UrhG, von Kommunikationspartnern empfangene Nachrichten sind bereits nicht vom Erblasser geschaffen im Sinne von $\$ 7$ UrhG. Zugleich dürfte eine Mehrzahl an Accounts (zumindest auch) geschütztes Material enthalten. Das gilt unter dem Gesichtspunkt des Leistungsschutzes, in dessen Rahmen $\$ 2$ Abs. 2 UrhG nicht eingreift, insbesondere für die eingebrachten Bilder und Videos, soweit sie vom Nutzer selbst hergestellt wurden. Das Urheberrecht hat als Anknüpfungspunkt für die Berechtigung an den Accountinhalten gegenüber einem nutzungsvertraglichen Zugangsanspruch mithin jedenfalls den Nachteil der Unvollständigkeit der umfassten Inhalte.

\section{Vererbung des Urheberrechts, $\mathbb{} 28$ Abs. 1 UrhG}

Nach $\$ 28$ Abs. 1 UrhG ist das Urheberrecht vererblich. Dasselbe gilt für das Leistungsschutzrecht an Licht- und Laufbildern. ${ }^{767}$ Damit wird die Regelung des $\mathbb{} 1922$ Abs. 1 BGB speziell für das Erbe an Urheberrechten bekräftigt. Ausweislich des $\$ 30$ UrhG gehen grundsätzlich alle Rechte des Urhebers unter Einschluss der Urheberpersönlichkeitsrechte auf die Erben über. ${ }^{768}$ Insofern unterscheidet sich das Urheberpersönlichkeitsrecht von dem allgemeinen Persönlichkeitsrecht aus Art. 2 Abs. 1 GG in Verbindung

767 Obly in: Schricker/Loewenheim (Hrsg.), \$28 UrhG Rn. 18 (Lichtbildschutz), Rn. 20 (Laufbildschutz); J.B. Nordemann in: Fromm/Nordemann, $\mathbb{S} 28$ UrhG Rn. 16; Rebbinder/Peukert, Urheberrecht, Rn. 991. Die nachfolgenden Ausführungen zum UPR sind für das Leistungsschutzrecht indes nicht relevant, weil Leistungsschutzrechte nicht in demselben Maße persönlichkeitsrechtlich geprägt sind wie Urheberrechte.

768 Obly in: Schricker/Loewenheim (Hrsg.), $\$ 28$ UrhG Rn. 1 m.w.N.; Schack, Urheber- und Urhebervertragsrecht, Rn. 648 ff.; J.B. Nordemann in: Fromm/Nordemann, $\mathbb{2} 28$ UrhG Rn. 1; Rehbinder/Peukert, Urheberrecht, Rn. 988; zu beachten sind indes die den Rechtsnachfolger betreffenden Besonderheiten aus $\mathbb{S} \$ 31 \mathrm{a}$ 
mit Art. 1 Abs. 1 GG, welches nur in seinen vermögenswerten Bestandteilen vererblich ist und hinsichtlich der ideellen Teile als postmortales Persönlichkeitsrecht von den Angehörigen subjektlos treuhänderisch für den Verstorbenen ausgeübt wird. ${ }^{769}$ Das Urheberrecht hingegen bleibt auf Grundlage der monistischen Theorie des deutschen Urheberrechts ${ }^{770}$ auch im Erbgang ein einheitliches Recht. ${ }^{771}$ Bei der Ausübung des übergegangenen Urheberrechts sind die Rechtsnachfolger nicht an den Willen des Urhebers gebunden, ${ }^{772}$ weshalb sie ihr nunmehr eigenes Urheberrecht folglich nicht selbst durch eine interessenwidrige Ausübung verletzen können. ${ }^{773}$

Hieraus ergibt sich der Unterschied zum allgemeinen Persönlichkeitsrecht: Verletzen die Erben das postmortale Persönlichkeitsrecht des Erblassers, können sie von den wahrnehmungsberechtigten Angehörigen des Erblassers auf Unterlassung in Anspruch genommen werden. ${ }^{774}$ Hingegen

Abs. 2 S. 3, 42 Abs. 1 S. 2, 62 Abs. 5 S. 2, 115 ff. UrhG, vgl. dazu Schack a.a.O. Rn. 649 und Spautz/Götting in: BeckOK Urheberrecht, $\mathbb{} 30$ UrhG Rn. 4.

769 Spautz/Götting in: BeckOK Urheberrecht, $\$ 28$ UrhG Rn. 3 sehen wegen dieser Unterscheidung eine eigenständige Bedeutung von $\mathbb{2} 28$ UrhG; a.A. ( $\mathbb{2} 28$ UrhG sei rein deklaratorisch) Herzog/Pruns, Der digitale Nachlass in der Vorsorge- und Erbrechtspraxis, S. 24; zum allgemeinen Persönlichkeitsrecht im Erbgang vgl. die Nachweise in Fn. 378 und 379.

770 Dazu Schack, Urheber- und Urhebervertragsrecht, Rn. 343 („das Urheberrecht [ist] ein einheitliches Recht, in welchem persönlichkeits- und vermögensrechtliche Befugnisse untrennbar miteinander verwoben sind."); abgrenzend zur dualistischen Theorie Loewenheim/Peifer in: Schricker/Loewenheim (Hrsg.), $\$ 11$ UrhG Rn. 3.

771 Gloser, DNotZ 2013, 497, 501; Schack, Urheber- und Urhebervertragsrecht, Rn. 649; Wiebe in: Spindler/Schuster (Hrsg.), Recht der elektronischen Medien, $\$ 30$ UrhG Rn. 1; Ohly in: Schricker/Loewenheim (Hrsg.), $\$ 30$ UrhG Rn. 4.

772 Das gilt freilich nicht, sofern sich der Erblasser gegenüber Dritten vertraglich gebunden hat, vgl. Ohly in: Schricker/Loewenheim (Hrsg.), 30 UrhG Rn. 6. Zur Bindung der Erben an vom Erblasser eingeräumte Lizenzen siehe noch S. $205 \mathrm{f}$.

773 So die h.M., vgl. Obly in: Schricker/Loewenheim (Hrsg.), $\$ 30$ UrhG Rn. 6 f.; J.B. Nordemann in: Fromm/Nordemann, $\mathbb{3} 30$ UrhG Rn. 10; Schulze in: Dreier/Schulze (Hrsg.), UrhG, $\$ 30$ Rn. 4; Kotthoff in: Dreyer/Kotthoff/Meckel/Hentsch (Hrsg.), Urheberrecht, $\mathbb{3} 30$ UrhG Rn. 3; A. Nordemann in: Loewenheim (Hrsg.), Handbuch des Urheberrechts, $\mathbb{2} 23 \mathrm{Rn} .22$; a.A. (treuhänderische Bindung des Urheberpersönlichkeitsrechts vergleichbar dem APR) Clément, Urheberrecht und Erbrecht, S. 64 ff.; Rehbinder/Peukert, Urheberrecht, Rn.989; Schack, Urheber- und Urhebervertragsrecht, Rn. 651; von Welser, Die Wahrnehmung urheberpersönlichkeitsrechtlicher Befugnisse durch Dritte, S. $148 \mathrm{ff}$.

774 Vgl. dazu bereits die Nachweise in Fn. 405, 406 und 407. 
sind die Erben in ihrem Umgang mit den geschützten Werken des Erblassers grundsätzlich frei, weil sie selbst Inhaber des Urheberpersönlichkeitsrechts sind und niemand sonst aktivlegitimiert aus ebendiesem Recht gegen sie vorgehen kann. ${ }^{775}$ Wollen die Erben aber ihrerseits auf Grundlage des Urheberpersönlichkeitsrechts gegen Dritte vorgehen, sind für die erforderliche Interessenabwägung stets die Interessen des Urhebers maßgeblich. ${ }^{776}$ Beispielsweise setzen Ansprüche der Erben gegen Dritte wegen Entstellung des Werks gem. $\$ 14$ UrhG demnach voraus, dass die „berechtigten geistigen oder persönlichen Interessen" des Urhebers - und nicht etwa diejenigen der Erben - gefährdet sind. Das ist relevant für die Ausübung von jeglichen urheberpersönlichkeitsrechtlichen Befugnissen durch die Erben. ${ }^{777}$

\section{Zwischenergebnis zu I.}

Die urheberrechtliche Analyse des Erblasseraccounts ergibt, dass - in Abhängigkeit von den Umständen des Einzelfalls - an einer Vielzahl, nicht aber an allen eingebrachten Inhalten Urheber- oder Leistungsschutz nach Maßgabe des UrhG bestehen kann. Diese Rechte gehen allesamt und in vollem Umfang auf die Erben über. Vererblich ist indes nur das Urheberrecht, nicht hingegen die Urheberschaft. ${ }^{778}$ Sofern das Gesetz demnach einen Anspruch von (überwiegenden) urheberpersönlichkeitsrechtlichen Interessen des Urhebers abhängig macht, so bezieht sich das auf den verstorbenen Schöpfer und nicht auf den Rechtsnachfolger.

\section{Lizenz des Providers an Urheberrechten des Nutzers}

Damit der Provider die urheber- beziehungsweise leistungsschutzrechtlich geschützten Inhalte des Nutzers auf einem Server speichern und Dritten, wie den Privatkontakten oder Followern des Nutzers, zugänglich machen

775 Schack, Urheber- und Urhebervertragsrecht, Rn. 649 und dort in Fn. 11 und 12.

776 So die h.M, vgl. ebd. Fn. 773; a.A. (rein erbrechtliche Betrachtung: keine Unterscheidung zwischen Urheber- und Erbeninteressen) Gloser, Die Rechtsnachfolge in das Urheberrecht, S. $124 \mathrm{ff}$. und Gergen, ZErb 2009, 42, $47 \mathrm{f}$.

777 Zum Rückrufsrecht (der Erben) wegen gewandelter Überzeugung gem. $\mathbb{\$} 42$ Abs. 1 (S. 2) UrhG vgl. noch in Fn. 850.

778 So fast wörtlich Schulze in: Dreier/Schulze (Hrsg.), UrhG, $\mathbb{} 28$ Rn. 3; siehe ferner Spautz/Götting in: BeckOK Urheberrecht, $\mathbb{} 28$ UrhG Rn. 3. 
darf, muss der Provider Inhaber eines wirksam eingeräumten Nutzungsrechts ${ }^{779}$ sein. ${ }^{780}$ Die Praxis der Provider bestätigt diesen Befund, wie ein exemplarischer Blick auf die AGB der Anbieter Facebook ${ }^{781}$, YouTube ${ }^{782}$, LinkedIn $^{783}$, Instagram ${ }^{784}$ und Pinterest ${ }^{785}$ zeigt. Die Provider streben mit ihren AGB jeweils eine umfangreiche Einräumung von Nutzungsrechten an den geschützten Inhalten des Nutzers an. Besonders weit geht die entsprechende Klausel von Twitter. ${ }^{786}$ Vorgesehen ist dort eine „weltweite, nicht ausschließliche, unentgeltliche Lizenz mit dem Recht zur Unterlizenzierung, [nutzerseitig eingebrachte] Inhalte [...] über sämtliche Verbreitungswege, die gegenwärtig bekannt sind oder in Zukunft bekannt sein werden [...] zu verwenden, zu vervielfältigen, zu reproduzieren, zu verarbeiten, anzupassen, abzuändern, zu veröffentlichen, zu übertragen, anzuzeigen und zu verbreiten." ${ }^{\text {"787 }} \mathrm{Ob}$ und inwieweit die Lizenzierung mittels AGB gegenüber dem Urheber wirksam ist, muss am Übertragungszweckgedanken des $₫ 31$ Abs. 5 UrhG und an den $\$ \$ 305 \mathrm{ff}$. BGB gemessen werden (dazu 1.). Nach dem Erbfall stellt sich sodann die Frage, ob auch die Rechtsnachfolger und nunmehrigen Urheberrechtsinhaber an die Ein-

779 Vgl. die Legaldefinition in $\$ 31$ Abs. 1 S. 1 UrhG: „Der Urheber kann einem anderen das Recht einräumen, das Werk auf einzelne oder alle Nutzungsarten zu nutzen." Ausführlich zu dem Begriff Berger in: Berger/Wündisch (Hrsg.), Urhebervertragsrecht, $\mathbb{1} 1 \mathrm{Rn} .38 \mathrm{ff}$.

780 Betroffen sind mind. $\mathbb{S}$ 16, 19a und ggf. 23 S. 1 UrhG, s. Berberich, MMR 2010, 736, 737, 739; Paul in: Hoeren/Sieber/Holznagel (Hrsg.), MMR-HdB, Teil 7.4 Rn. 140; Solmecke ebd., Teil 21.1 Rn. 6; ders./Dam, MMR 2012, 71, 72; ferner König/Stang in: Münchener Anwaltshandbuch Urheberrecht, $₫ 31$ Rn. 14.; zweifelnd aber Imhof in: Bisges (Hrsg.), Handbuch Urheberrecht, Kap. 4 Rn. 139.

781 Vgl. Ziff. 3.3.1 der AGB von Facebook, abrufbar a.a.O. (Fn. 636); die Wirksamkeit einer früheren Version der Klausel offenlassend BGH NJW 2018, 3178 Rn. 38.

782 Vgl. unter „Ihre Inhalte und Ihr Verhalten“ in den AGB von YouTube, abrufbar unter https://www.youtube.com/t/terms [17.11.2020].

783 Vgl. Ziff. 3.1 der AGB von LinkedIn, abrufbar a.a.O. (Fn. 655).

784 Vgl. unter „Deine Verpflichtungen“ in den AGB von Instagram, abrufbar unter https://help.instagram.com/581066165581870 [17.11.2020].

785 Vgl. Ziff. 3.b der AGB von Pinterest, abrufbar unter https://policy.pinterest.com /de/terms-of-service [17.11.2020].

786 Vgl. Ziff. 3 der AGB von Twitter, abrufbar unter https://twitter.com/de/tos [17.11.2020].

787 Ebd. (Fn. 786); die Klausel soll darüber hinaus explizit ein Recht zur unentgeltlichen Nutzung durch Twitter und „andere Unternehmen und Organisationen“ umfassen, die Inhalte des Nutzers zu Werbezwecken zu verwenden und in anderen Medien zu veröffentlichen; zu diesem Aspekt König/Stang in: Münchener Anwaltshandbuch Urheberrecht, $\$ 31$ Rn. 14 und dort in Fn. 46. 
räumung des Nutzungsrechts gebunden sind und inwieweit diese Bindung von der Stellung als Accountinhaber (un)abhängig ist (dazu 2.).

\section{Wirksame Vereinbarung über Nutzungsrechte}

Hinsichtlich des Prüfungsmaßstabs ist zwischen zwei unterschiedlichen Arten von Rechtseinräumungsklauseln zu unterscheiden. Für pauschal formulierte Klauseln, welche die eingeräumten „Nutzungsarten nicht ausdrücklich einzeln bezeichnen“, gilt unmittelbar $\$ 31$ Abs. 5 UrhG (dazu a). ${ }^{788}$ Dagegen verbleibt bei Klauseln, die wie etwa die beispielhaft zitierten AGB von Twitter alle erfassten Nutzungsarten katalogartig aufzählen, ${ }^{789}$ kein Raum für die Anwendung von $\$ 31$ Abs. 5 UrhG; sie sind (allein) an den $\$ \$ 305 \mathrm{ff}$. BGB zu messen (dazu b).

\section{a) Übertragungszweckgedanke des $₫ 31$ Abs. 5 UrhG}

Nach dem Übertragungszweckgedanken ${ }^{790}$ des $₫ 31$ Abs. 5 UrhG räumt der Urheber im Zweifel keine weitergehenden Rechte ein, als es der Zweck der Verfügung verlangt. ${ }^{791}$ Die Bestimmung des Verfügungszwecks muss dabei für den jeweiligen Internetdienst mit Blick auf seine Funktion für den Nutzer und seine technische Ausgestaltung erfolgen. ${ }^{792}$ Der zugrundeliegende Nutzungsvertrag indiziert insoweit den von beiden Parteien verfolgten Zweck: ${ }^{793}$ Im Vordergrund steht regelmäßig, die technische

788 Nordemann, NJW 2012, 3121, 3122; Kutscher, Der digitale Nachlass, S. 37; Berberich, MMR 2010, 736, 737; grundsätzlich zum Verhältnis von Urheberrecht und AGB-Kontrolle Mäsch in: Staudinger, Anhang zu $\$ \$ 305-310$ BGB Rn. L 31 ff.

789 Kritisch dazu Rebbinder/Peukert, Urheberrecht, Rn. 916 („Nutzungsrechtseinräumungen tendieren [...] heute zur Geschwätzigkeit amerikanischer Verträge“).

790 Früher Zweckübertragungstheorie genannt, vgl. zur Terminologie Schack, Urheber- und Urhebervertragsrecht, Rn. 615 und dort in Fn. 91 sowie Burda, Die Zweckbindung im Urhebervertragsrecht, S. $43 \mathrm{ff}$.

791 Wiebe in: Spindler/Schuster (Hrsg.), Recht der elektronischen Medien, $\mathbb{} 31$ UrhG Rn. 13; Schulze in: Dreier/Schulze (Hrsg.), UrhG, $\$ 31$ Rn. 110; einen Überblick über die Rechtsprechung geben Hertin/Wagner, Urheberrecht, Rn. 555 ff.; ausführlich Burda, Die Zweckbindung im Urhebervertragsrecht, S. $52 \mathrm{ff}$.

792 Berberich, MMR 2010, 736, 737 („einzelfallabhängig“); zur Bestimmung des Zwecks Burda, Die Zweckbindung im Urhebervertragsrecht, S. $112 \mathrm{ff}$.

793 Burda, Die Zweckbindung im Urhebervertragsrecht, S. $116 \mathrm{f}$. 
und wirtschaftliche Funktionsfähigkeit der Plattform sicherzustellen, um die Nutzung der Plattform zu ermöglichen - nicht jedoch die Einräumung von Nutzungsrechten zum Zwecke der sonstigen Verwertung. ${ }^{794}$ Danach würde im Falle einer pauschal formulierten Rechtseinräumungsklausel ${ }^{795}$ nach Maßgabe von $\$ 31$ Abs. 5 UrhG (nur) ein einfaches, auf die Laufzeit des Nutzungsvertrags begrenztes Recht des Providers nach $\$ 16$ UrhG (Vervielfältigungsrecht) und $\$ 19$ a UrhG (Recht der öffentlichen Zugänglichmachung) sowie (in Abhängigkeit von den Funktionen der Plattform) ggf. nach $₫ 23$ S. 1 UrhG (Bearbeitungsrecht) eingeräumt. ${ }^{796}$ Darüber hinaus muss der Provider diese Rechte an den Werken des Erblassers seinerseits Dritten einräumen können, wenn und soweit die Plattform vorsieht, dass andere Nutzer die Inhalte des Erblassers downloaden und selbst weiterbearbeiten können. ${ }^{797}$

Indessen greift der Übertragungszweckgedanke aufgrund seiner Rechtsnatur als Auslegungsregel lediglich, wenn und soweit Raum für eine Auslegung besteht. ${ }^{798}$ Die aktuellen AGB der Provider enthalten keine pauschalen, sondern sehr detaillierte Rechtseinräumungsklauseln. ${ }^{799}$ Damit vermeidet die Providerpraxis gerade die Erfüllung des negative Tatbestandsmerkmals aus $\$ 31$ Abs. 5 S. 1 UrhG, wonach die vom Nutzungsrecht erfassten Nutzungsarten "nicht ausdrücklich einzeln bezeichnet" ${ }^{\text {800 }}$ sein dürfen. Die Wirksamkeit detaillierter Klauseln zur Nutzungsrechtseinräumung ist demnach allein am Maßstab der $\$ \$ 305 \mathrm{ff}$. BGB zu prüfen.

794 Berberich, MMR 2010, 736, 737; in diese Richtung auch Solmecke/Dam, MMR 2012, 71, 73 f.; Kutscher, Der digitale Nachlass, S. 35.

795 Vgl. jeweils mit einem Beispiel für eine solche Klausel Nordemann/Czychowski in: Münchener Anwaltshandbuch Gewerblicher Rechtsschutz, $\$ 43$ Rn. 137 und Berberich, MMR 2010, 736, 737.

796 Ähnlich (aber über $₫ 307$ Abs. 2 Nr. 1 BGB) Solmecke/Dam, MMR 2012, 71, 72.

797 Redeker in: Hoeren/Sieber/Holznagel (Hrsg.), MMR-HdB, Teil 12 Rn. 432 a.E.

798 Berberich, MMR 2010, 736, 737; Soppe in: BeckOK Urheberrecht, $\mathbb{3} 1$ UrhG Rn.99; Solmecke in: Hoeren/Sieber/Holznagel (Hrsg.), MMR-HdB, Teil 21.1 Rn. 8; s. aber Rehbinder/Peukert, Urheberrecht, Rn. 916.

799 Vgl. die Beispiele a.a.O. (Fn. 781-786).

$800 \rrbracket 31$ Abs. 5 S. 1 UrhG lautet: „Sind bei der Einräumung eines Nutzungsrechts die Nutzungsarten nicht ausdrücklich einzeln bezeichnet, so bestimmt sich nach dem [...] Vertragszweck, auf welche Nutzungsarten es sich erstreckt." (Hervorhebung nicht im Original). 
Die Klauseln sind Bestandteil der für eine Vielzahl von Verträgen vorformulierten Vertragsbedingungen, welche der Provider als Verwender dem Nutzer stellt im Sinne von $₫ 305$ Abs. 1 S. 1 BGB. Hinsichtlich der Anforderungen an eine wirksame Einbeziehung insbesondere gem. $\$ 305$ Abs. 2 Nr. 1 und 2 BGB gelten die Ausführungen zu AGB-Klauseln betreffend die Vererbbarkeit des Vertragsverhältnisses entsprechend. ${ }^{801}$ Ein spezifischer Aspekt der Einbeziehungskontrolle von Rechtseinräumungsklauseln wird unter dem Gesichtspunkt des $₫ 305$ c Abs. 1 BGB diskutiert. ${ }^{802}$ Danach werden Bestimmungen in AGB, „die so ungewöhnlich sind, dass der Vertragspartner des Verwenders mit ihnen nicht zu rechnen braucht, $[\ldots]$ nicht Vertragsbestandteil.“ Zur Annahme einer solchen „überraschenden Klausel“ muss der Art oder dem Umfang der Nutzungsrechtseinräumung - aus Sicht eines Durchschnittsurhebers - ein Überrumpelungseffekt zukommen. ${ }^{803}$ Zwar werden die weite Verbreitung der vorliegenden Klauseln im Kontext sozialer Medien sowie die damit einhergehende Aufmerksamkeit gegen ein Hindernis für die Einbeziehung im Sinne von $\$ 305$ c Abs. 1 BGB ins Feld geführt. ${ }^{804}$ Demgegenüber ist aber fraglich, ob die angesprochenen Verkehrskreise tatsächlich von der Verwendung solcher Klauseln ausgehen und ob in Ansehung der großen Vielfalt der Anbieter und ihrer jeweiligen Klauseln von einer „Branchenüblichkeit“ gesprochen werden kann. ${ }^{805}$ Sofern im Einzelfall eine Klausel zur Rechtseinräumung, etwa aufgrund ihres Umfangs, als überraschend gem. $\$ 305$ c Abs. 1 BGB einzuordnen ist, richtet sich die Lizenzierung gem. $\$ 306$ Abs. 1 und 2 nach der gesetzlichen Vorschrift des $\$ 31$ Abs. 5 UrhG. ${ }^{806}$ Insoweit kann dann auf

801 Siehe dazu zuvor auf S. $162 \mathrm{f}$. Indes ist die dort problematische Regelung jenseits der AGB im Hilfe-Bereich mancher sozialer Netzwerke für die hier relevante Einräumung von Nutzungsrechten unüblich, vgl. die Klauselbeispiele a.a.O. (Fn. 781-786), die allesamt ein regulärer Bestandteil der jeweiligen AGB sind.

802 Vgl. die Nachweise in Fn. 804.

803 Wandtke/Grunert in: Wandtke/Bullinger (Hrsg.), UrhG, Vor $\$ \$ 31 \mathrm{ff}$. Rn. 103; Obly in: Schricker/Loewenheim (Hrsg.), Vor $\$ \$ 31 \mathrm{ff.}$ UrhG Rn. 40.

804 Berberich, MMR 2010, 736, 737; Kutscher, Der digitale Nachlass, S. 32 f.; i.E. auch Imbof in: Bisges (Hrsg.), Handbuch Urheberrecht, Kap. 4 Rn. 138; a.A. Solmecke/ Dam, MMR 2012, 71, 72; (wohl) Nordemann, NJW 2012, 3121, 3121 und Obly in: Schricker/Loewenheim (Hrsg.), Vor $\$ \$ 31 \mathrm{ff}$. UrhG Rn. 40

805 Solmecke/Dam, MMR 2012, 71, 72.

806 Ebd. (Fn. 805). 
die Ausführungen zu pauschal formulierten Rechtseinräumungsklauseln verwiesen werden. 807

Wirksam einbezogene AGB sind sodann einer Inhaltskontrolle nach $\$ \$ 307 \mathrm{ff}$. BGB zu unterziehen, sofern deren Anwendungsbereich gem. $\$ 307$ Abs. 3 S. 1 BGB eröffnet ist. Die Kontrollfähigkeit der Rechtseinräumung in urheberrechtlichen Lizenzverträgen wird vereinzelt bestritten, weil die Hauptleistungspflicht des Urhebers der gerichtlichen Kontrolle entzogen sein soll. ${ }^{808}$ Diese Argumentation kann jedoch nicht auf die Nutzungsverträge zwischen einem Internetdiensteanbieter und seinen Nutzern übertragen werden, weil die Einräumung von Nutzungsrechten in diesem Fall gerade nicht die Hautpflicht ist, sondern als Nebenpflicht lediglich die Teilnahme an der Plattform ermöglicht. 809

Bei der demnach eröffneten Inhaltskontrolle sind die Lizenzklauseln auf eine unangemessene Benachteiligung des Nutzers hin zu überprüfen. Dabei ergeben sich vor allem aus $₫ 307$ Abs. 2 Nr. 1 BGB (dazu aa) und dem Transparenzgebot ${ }^{810}$ des $\$ 307$ Abs. 1 S. 2 BGB (dazu bb) die wesentlichen Vorgaben für wirksame Bestimmungen zur Rechtseinräumung in AGB (dazu cc).

aa) Leitbildverstoß, $₫ 307$ Abs. 2 Nr. 1 BGB

Für die Annahme eines Leitbildverstoßes im Sinne von $₫ 307$ Abs. 2 Nr. 1 BGB kommen zwei gesetzliche Ausgangspunkte in Betracht, von denen die Provider mit ihrer Regelungspraxis abweichen. Das könnte zum einen der Übertragungszweckgedanke des $₫ 31$ Abs. 5 UrhG sein, der insoweit jenseits seines originären Anwendungsbereichs auch bei detailliert ausformulierten Klauseln in der Gestalt als gesetzliches Leitbild über $\$ 307$ Abs. 2 Nr. 1 BGB in die Debatte zurückkehrt. Zum anderen könnte es $\mathbb{1 1}$ S. 2

807 Siehe dazu S. $196 \mathrm{f}$.

808 Schack, Urheber- und Urhebervertragsrecht, Rn. 1087; ausführlich dazu Peifer, AfP 2012, 510, $514 \mathrm{f}$.

809 Berberich, MMR 2010, 736, 738; Kutscher, Der digitale Nachlass, S. 33 f.; Obly in: Schricker/Loewenheim (Hrsg.), Vor $\$ \$ 31 \mathrm{ff}$. UrhG Rn. 49; Solmecke/Dam, MMR 2012, 71, 72; nunmehr auch Schack, Urheber- und Urhebervertragsrecht, Rn. 1087 in Fn. 37.

810 Insoweit kommt es auf die festgestellte Eröffnung der Inhaltskontrolle gem.

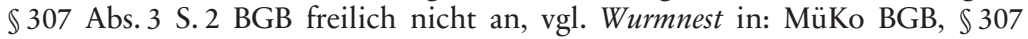
Rn. 22. 
UrhG sein, wonach das Urheberrecht (auch) „der Sicherung einer angemessenen Vergütung für die Nutzung des Werkes"dienen soll.

Zwar hat der BGH zu $₫ 31$ Abs. 5 UrhG in einer Grundsatzentscheidung $^{811}$ festgehalten, dass dem Übertragungszweckgedanken als (schlichter) Auslegungsregel kein Leitbildcharakter im Sinne von $\$ 307$ Abs. 2 Nr. 1 BGB zukommt. ${ }^{812}$ Indes ist es (selbst ungeachtet der grundsätzlichen Kritik an dem Urteil im urheberrechtlichen Schrifttum) ${ }^{813}$ fraglich, ob die Ausführungen des BGH für Nutzungsverträge zwischen Providern und ihren Nutzern gelten können. ${ }^{814}$ Das wesentliche Argument des BGH entspringt dem Kontext klassischer urheberrechtlicher Lizenzverträge, die von Urhebern und Verwertern in gewerblicher Absicht abgeschlossen werden: Die von $\$ 11$ S. 2 UrhG verlangte Angemessenheit der Vergütung des Urhebers möchte der BGH sicherstellen, indem die vom Verwerter erbrachte finanzielle Gegenleistung für die Nutzungsrechtseinräumung erforderli-

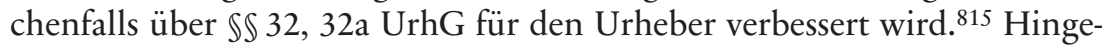
gen stehe einer Kontrolle des Umfangs der Nutzungsrechtseinräumung als Hauptleistungspflicht des Urhebers der Vorrang der privatautonomen Vertragsgestaltung entgegen. ${ }^{816}$

Der Nutzer sozialer Medien hingegen räumt dem Provider gerade unentgeltlich Nutzungsrechte an seinen Inhalten ein. Eine Korrektur allein auf der Vergütungsseite im Sinne des BGH würde eine von den Parteien nicht vorgesehene wirtschaftliche Verwertung der Inhalte überhaupt erst begründen. ${ }^{817}$ Wenn aber eine finanzielle Kompensation des Nutzers für die über den Vertragszweck hinausgehende Rechtseinräumung nicht möglich

811 BGH GRUR 2012, 1031 Rn. 16 ff.; instruktiv zu den $\$ 31$ Abs. 5 UrhG betreffenden Aussagen des Urteils vgl. Schippan, ZUM 2012, 771, 773 f; das Gericht setzt insoweit die frühere Rechtsprechung aus BGH GRUR 1984, 45, $48 \mathrm{f}$. fort

812 Ebenso Nordemann, NJW 2012, 3121, 3122 und Wille, ZUM 2011, 206, $206 \mathrm{ff}$.

813 Wandtke/Grunert in: Wandtke/Bullinger (Hrsg.), UrhG, Vor $\$ \$ 31$ ff. Rn. 108; Obly in: Schricker/Loewenheim (Hrsg.), Vor $\mathbb{S} 31 \mathrm{ff}$. UrhG Rn. 49; Schulze in: Dreier/Schulze (Hrsg.), UrhG, Vor $\$ 31$ Rn. 16; ders., GRUR 2012, 993, 993 f.; Berberich, WRP 2012, 1055, $1057 \mathrm{ff}$.

814 Insoweit ebenso zweifelnd Berberich, MMR 2010, 736, 739 und Kutscher, Der digitale Nachlass, S. 34 f.; a.A. (wohl) Paul in: Hoeren/Sieber/Holznagel (Hrsg.), MMR-HdB, Teil 7.4 Rn. 146.

815 BGH GRUR 2012, 1031 Rn. 29; Wille, ZUM 2011, 206, 209; Dorner, MMR 2011, $780,782$.

816 BGH GRUR 2012, 1031 Rn. 18; auch Imbof in: Bisges (Hrsg.), Handbuch Urheberrecht, Kap. 4 Rn. 81 betont gerade die Eigenschaft als Hauptleistungspflicht.

817 Ebd. (Fn. 814). 
ist, dann kann dem zwingenden Gebot des $\$ 11$ S. 2 UrhG nur über eine Kontrolle des Umfangs der eingeräumten Rechte genügt werden. ${ }^{818}$

Folglich begrenzt der Zweck des Nutzungsvertrags gem. $\$ 307$ Abs. 2 Nr. 1 BGB die Reichweite des dem Provider eingeräumten Nutzungsrechts. ${ }^{819}$ Als Vertragszweck wiederum kommt nur in Betracht, was beide Parteien gemeinsam anstreben oder zumindest akzeptieren, nicht aber einseitige Zwecksetzungen (des Providers). ${ }^{820}$ Eine Zweckbestimmung in AGB ist ihrerseits am Transparenzgebot des $\$ 307$ Abs. 1 S. 2 BGB zu messen.

bb) Transparenzgebot, $₫ 307$ Abs. 1 S. 2 BGB

Gem. \307 Abs. 1 S. 2 BGB kann sich eine unangemessene Benachteiligung des Vertragspartners des Verwenders „auch daraus ergeben, dass die Bestimmung nicht klar und verständlich ist." Um dem Transparenzgebot zu genügen, muss der Verwender die Rechte und Pflichten seines Vertragspartners so klar wie möglich formulieren. ${ }^{821}$ Über die schlichte sprachliche Verständlichkeit hinaus schließt das ebenso ein, dass ein durchschnittlicher Vertragspartner die (wirtschaftlichen) Belastungen, die sich aus den AGB ergeben (können), nachvollziehen kann. ${ }^{822}$ Weder soll die Umschreibung Auslegungsspielräume einseitig zugunsten des Verwenders eröffnen, noch darf sie den Vertragspartner durch falsche Vorstellungen vom Vertragsinhalt zu einem unangemessenen Vertragsabschluss bringen. ${ }^{823}$

Im Folgenden sollen vor allem die Klauseln der Provider zur Bestimmung des Zwecks der Nutzungsrechtseinräumung im Lichte des Transpa-

818 Im Ergebnis wie hier, aber allein über $₫ 11$ S. 2 UrhG argumentiert das KG Berlin ZD 2014, 412, $417 \mathrm{f}$. In der Revision musste BGH ZD 2016, 484 dazu nicht Stellung nehmen; s. außerdem bereits die Nachweise in Fn. 814 sowie Schack, Urheber- und Urhebervertragsrecht, Rn. 774a.

819 Zum Zweck von Nutzungsverträgen vgl. bereits zuvor auf S. $196 \mathrm{f}$.

820 Obly in: Schricker/Loewenheim (Hrsg.), $\$ 31$ UrhG Rn. 64 f. möchte zur Ermitt-

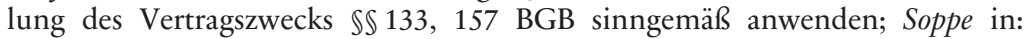
BeckOK Urheberrecht, $\$ 31$ UrhG Rn. 95 ff.; Schulze in: Dreier/Schulze (Hrsg.), UrhG, $\mathbb{3} 31$ Rn.121f.; ausführlich zur Zweckbestimmung Burda a.a.O. (Fn. 792).

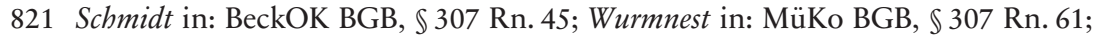
Grüneberg in: Palandt, $₫ 307$ BGB Rn. 21; Schulte-Nölke in: HK-BGB, $₫ 307$ BGB Rn. 21 f. jeweils m.w.N.

822 Ebd. (Fn. 821).

823 Ebd. (Fn. 821). 
renzgebots betrachtet werden. ${ }^{824}$ Etwa die AGB der Anbieter Instagram ${ }^{825}$, Facebook ${ }^{826}$ und Google ${ }^{827}$ enthalten solche Zweckbestimmungen, während beispielsweise Twitter ${ }^{828}$ auf eine explizite Spezifizierung verzichtet. Wenn der Zweck so formuliert ist, dass die Rechtseinräumung eine Bedingung dafür sein soll, dass der Dienst zur Verfügung gestellt werden kann, ${ }^{829}$ dann muss unterschieden werden: Soll die Rechtseinräumung in einem allgemeineren Sinne den Betrieb des Dienstes und seine Weiterentwicklung ermöglichen (d.h. insbesondere finanzieren), sodass auch eine weitgehende gewerbliche Verwertung der Inhalte etwa zu Werbezwecken von diesem Zweck erfasst ist. Oder ist in einem engeren technisch-funktionalen Sinne gemeint, dass die Funktionen der Plattform (wie beispielsweise das „Posten“ und „Teilen“ von Inhalten) einem Nutzer gegenüber in urheberrechtlich zulässiger Weise erbracht werden können. Um dem Transparenzgebot zu genügen, müssen die Formulierung und der Kontext in den AGB verdeutlichen, welche der beiden Varianten gemeint ist. Denn die rechtlichen Konsequenzen für die Reichweite der eingeräumten Nutzungsrechte unterscheiden sich deutlich und der Nutzer muss nachvollzie-

824 U.a. zur Intransparenz, die (bei ausländischen Internetdiensteanbietern) aus Übersetzungsproblemen resultieren kann, vgl. Solmecke/Dam, MMR 2012, 71, 72, 74; allgemein auch Nordemann, NJW 2012, 3121, 3125 a.E. („Der Einhaltung des Transparenzgebots muss in urheberrechtlichen Formularverträgen besondere Aufmerksamkeit geschenkt werden.").

825 Vgl. unter „Deine Verpflichtungen“ in den AGB von Instagram, abrufbar a.a.O. (Fn. 784): Du „gewährst [...] uns hiermit [...] eine [...] Lizenz, [...] damit wir den Instagram-Dienst zur Verfügung stellen können" (Hervorhebung nur hier).

826 Vgl. Ziff. 3.3.1 der AGB von Facebook, abrufbar a.a.O. (Fn. 636): „Diese Lizenz dient nur dem Zweck, dir unsere Produkte bereitzustellen.“ In einer früheren Version der Zweckbestimmungsklausel von Facebook sah das KG Berlin ZD

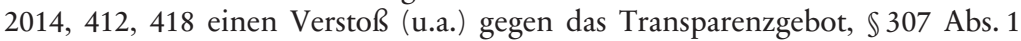
S. 2 BGB.

827 Vgl. unter „Lizenz“ in den AGB von Google, abrufbar unter https://policies.goo gle.com/terms?gl=DE\&hl=de [17.11.2020] („Diese Lizenz ist beschränkt auf den ausschließlichen Zweck der Erbringung der Dienste, das heißt um zu ermöglichen, dass die Dienste wie vorgesehen funktionieren und neue Funktionen und Funktionalitäten geschaffen werden können. Dies umfasst die Verwendung automatisierter Systeme und Algorithmen zur Analyse Ihrer Inhalte [...]“).

828 Vgl. Ziff. 3 der AGB von Twitter, abrufbar a.a.O. (Fn. 786).

829 Explizit so Instagram (s. die Hervorhebung in Fn. 825) und Google („um zu ermöglichen, dass die Dienste [...] funktionieren und neue [...] geschaffen werden können." Hervorhebung nur hier). 
hen können, inwieweit genau er die Verwertung seiner Werke gestattet. ${ }^{830}$ Es darf insoweit kein Auslegungsspielraum verbleiben, den der Provider zu seinen Gunsten nutzen kann, indem er zusätzliche Nutzungsarten als von der Zweckbestimmung erfasst definiert.

cc) Vorgaben für eine zulässige Klausel zur Rechtseinräumung

Nach alledem ergeben sich aus $₫ 307$ Abs. 1 S. 2 und Abs. 2 Nr. 1 BGB die Rahmenbedingungen für eine zulässige Klausel, mittels welcher die unentgeltliche Einräumung von Nutzungsrechten an den Inhalten eines Nutzers sozialer Medien vereinbart wird. Die Reichweite der Rechtseinräumung wird durch den Vertragszweck begrenzt. Dieser Zweck der Rechtseinräumung muss zwar für jeden Internetdienst in Abhängigkeit von dessen Funktionen jeweils einzeln bestimmt werden, wird aber regelmäßig primär in der technisch-funktionalen Ermöglichung des Plattformbetriebs bestehen. Während die einseitige Festsetzung eines deutlich weitergehenden Zwecks in den AGB des Providers an $\ 307$ Abs. 2 Nr. 1 BGB scheitert, steht $₫ 307$ Abs. 1 S. 2 BGB einer mehrdeutigen Formulierung der Zweckbestimmung entgegen, die Auslegungsspielräume zugunsten des Providers belässt.

Aus dem vom Nutzungszweck verlangten direkten Bezug zum Plattformbetrieb ergeben sich Beschränkungen der Rechtseinräumung in inhaltlicher wie in zeitlicher Hinsicht. Die inhaltliche Dimension begrenzt die von der Lizenz erfassten Nutzungsarten und die Reichweite des providerseitigen Rechts zur Unterlizenzierung. ${ }^{831}$ Mit Blick auf die zeitliche Dimension scheiden überschießende Rechte über das Ende der Plattformnutzung hinaus aus. ${ }^{832}$ In den AGB einiger Anbieter finden sich bereits kon-

830 Paul in: Hoeren/Sieber/Holznagel (Hrsg.), MMR-HdB, Teil 7.4 Rn. 146; zwar auf $\$ 307$ Abs. 2 Nr. 2 BGB bzw. $\$ 305$ c Abs. 1 BGB abstellend betont aber auch Redeker in: Hoeren/Sieber/Holznagel (Hrsg.), MMR-HdB, Teil 12 Rn. 433, dass „der Umfang der Rechteeinräumung für den Nutzer klar erkennbar sein" muss.

831 Berberich, MMR 2010, 736, 738 mit Beispielen für insoweit „unnötige Rechte“; Schack, Urheber- und Urhebervertragsrecht, Rn. 774a; Paul in: Hoeren/Sieber/ Holznagel (Hrsg.), MMR-HdB, Teil 7.4 Rn. 146.

832 Berberich, MMR 2010, 736, 738; Paul in: Hoeren/Sieber/Holznagel (Hrsg.), MMR-HdB, Teil 7.4 Rn. 146. 
krete Anhaltspunkte für die Umsetzung dieser Maßstäbe, ${ }^{833}$ während andere Anbieter insoweit weder eine Beschränkung des Nutzungszwecks noch der eingeräumten Nutzungsrechte erkennen lassen. ${ }^{834}$ Die Einräumung eines einfachen, auf die Laufzeit des Nutzungsvertrags begrenzten Rechts des Providers nach $\$ \$ 16,19 \mathrm{a}$ und ggf. 23 S. 1 UrhG sowie die entsprechende Möglichkeit zur Unterlizenzierung bleiben demnach unproblematisch möglich.

c) Zwischenergebnis zu 1.

Sogenannte Buy-Out-Klauseln, mit denen der Urheberrechtsinhaber gegen einen Pauschalbetrag umfassend Nutzungsrechte an seinen Werken einräumt, sind in der Praxis verbreitet und bis zur Grenze des $₫ 138$ BGB zulässig. ${ }^{835}$ Indessen sind die dazu entwickelten Maßstäbe nur bedingt übertragbar auf Lizenzklauseln in Nutzungsverträgen der Anbieter von Kommunikations- und Informationsdiensten. Die Rechtseinräumung durch den Nutzer erfolgt hier im Gegensatz zu den Buy-Out-Klauseln im klassischen Urhebervertragsrecht erstens unentgeltlich und zweitens (lediglich) als Nebenleistung, sodass der Urheber und Social-Media-Nutzer nicht auf die Ansprüche auf angemessene Vergütung nach $\$ \mathbb{S 3 2}$, 32a UrhG verwiesen werden kann. Die daraus resultierende Notwendigkeit, die Reichweite der Rechtseinräumung selbst einer Kontrolle zu unterziehen, verleiht dem Urhebervertragsrecht insoweit eine verbraucherschützende Dimension. ${ }^{836}$ Das ist bemerkenswert, weil der Schutz des Urhebers als Kunstschaffendem und der Schutz des Verbrauchers als Rezipienten bislang meist gegen-

833 Beispielsweise findet sich eine explizite Begrenzung der Nutzungsrechtseinräumung in zeitlicher Hinsicht bei Facebook (s. Fn. 781), Instagram (s. Fn. 784) und Google (s. Fn. 827).

834 An der Wirksamkeit der AGB von Twitter, wonach ein Recht auf kommerzielle Verwertung aller Inhalte des Nutzers durch Twitter und seine Unterlizenznehmer vorgesehen ist (s. Fn. 786 und 787), bestehen insofern Zweifel.

835 So Schack, Urheber- und Urhebervertragsrecht, Rn. 1075, 1096 und Imhof in: Bisges (Hrsg.), Handbuch Urheberrecht, Kap. 4 Rn. 42 jeweils mit Verweis auf BGH GRUR 2012, 1031.

836 So bereits Berberich, MMR 2010, 736, 741 a.E. 
läufig wahrgenommen wurden ${ }^{837}$ diese Rollenverteilung im Kontext von nutzergenerierten Inhalten aber ihre Eindeutigkeit verliert. ${ }^{838}$

\section{Bindung der Rechtsnachfolger an die Lizenzeinräumung}

Wenn und soweit der Urheber zu seinen Lebzeiten wirksam Nutzungsrechte einräumt, sind die Erben ${ }^{839}$ als Rechtsnachfolger und neue Urheberrechtsinhaber gegenüber dem Lizenznehmer grundsätzlich ebenso an diese Rechtseinräumung gebunden. ${ }^{840}$ Dementsprechend konstatiert Obly, dass der „Rechtsnachfolger [...] im Blick auf den Sukzessionsschutz so behandelt [wird], wie wenn er das fragliche Nutzungsrecht selbst eingeräumt hätte. “841 Bezogen auf das Verhältnis zwischen Nutzer und Provider bleibt die Lizenz des Providers damit grundsätzlich nach Maßgabe der inhaltlichen und zeitlichen Beschränkungen, die sich aus der formularvertraglichen Einräumung ergeben, ${ }^{842}$ über den Erbfall hinaus weiter wirksam.

Inwiefern sich diese Beschränkungen auswirken, hängt wegen ihres Ursprungs im Zweck der Rechtseinräumung nicht zuletzt von dem Schicksal der Accountinhaberschaft im Erbfall ab. Wenn und soweit die Erben den Erblasseraccount nach dem Erbfall übernehmen und fortgesetzt nutzen, bleibt parallel dazu der Zweck der Nutzungsrechtseinräumung - die Ermöglichung des Plattformbetriebs - weiterhin gültig. Haben sich der Erb-

837 Vgl. dazu etwa Stieper, VuR 2014, 261, 261 f.; Wandtke in: Wandtke/Bullinger (Hrsg.), UrhG, Einleitung Rn. 26.

838 Bauer, User Generated Content, in: Große Ruse-Khan/Klass/v. Lewinski (Hrsg.), Nutzergenerierte Inhalte als Gegenstand des Privatrechts, S. 35 ff.; vgl. ferner die modellhafte Bezeichnung des „Prosument“ im Vorwort desselben Sammelbandes.

839 Zum Sukzessionsschutz auch bei einem Vermächtnisnehmer als Rechtsnachfolger im Urheberrecht, vgl. Gloser, Die Rechtsnachfolge in das Urheberrecht, S. 228.

840 Das insoweit einhellige Ergebnis wird in der Literatur auf unterschiedliche Normen gestützt, vgl. Gloser, Die Rechtsnachfolge in das Urheberrecht, S. 227 f. und Reinke in: Bisges (Hrsg.), Handbuch Urheberrecht, Kap. 9 Rn. 13 ( $\$ 33$ S. 2 Fall 1 UrhG); Obly in: Schricker/Loewenheim (Hrsg.), $\$ 33$ UrhG Rn. 16 und Wandtke, Urheberrecht, Kap. 4 Rn. 143 ( $\$ 30$ UrhG); Schack, Urheber- und Urhebervertragsrecht, Rn. 654 a.E. (S\$ 1922, 1967 Abs. 1 BGB); Rebbinder/Peukert, Urheberrecht, Rn. 988 (bürgerliches Recht); nicht festgelegt Berger in: Berger/Wündisch (Hrsg.), Urhebervertragsrecht, $\mathbb{1} 1$ Rn. 69 und Clément, Urheberrecht und Erbrecht, S. 104.

841 Obly in: Schricker/Loewenheim (Hrsg.), $\$ 33$ UrhG Rn. 16.

842 Vgl. dazu zuvor auf S. $198 \mathrm{ff}$. 
lasser und der Provider hingegen wirksam darauf geeinigt, dass der Account im Erbfall zu löschen ist, entfällt mit dem Tod des Nutzers der Lizenzzweck mit der Folge, dass das urheberrechtliche Nutzungsrecht des Providers endet. ${ }^{843}$

Betreibt schließlich eine vom Erblasser benannte Vertrauensperson, die nicht zugleich Erbin ist, den Account als eigenen oder als Gedenkseite weiter, fallen die Inhaberschaft von Account und Urheberrecht auseinander. In diesem Fall muss der Zweck der Rechtseinräumung wie folgt erneut in den Blick genommen werden. Die ursprünglichen Parteien des AccountVertrags (i.e. der Erblasser und der Provider) haben sich zum einen auf eine Einräumung von Nutzungsrechten zur Ermöglichung des Accountbetriebs und zum anderen auf die Berechtigung der Vertrauensperson am Account im Erbfall geeinigt. Vor diesem Hintergrund sind die Erben an die Urheberrechtslizenz des Providers gebunden, obwohl sie selbst keine Accountberechtigung haben. Denn der Lizenzzweck erfasst nach dem Willen der Vertragsparteien über die Lebzeiten des Erblassers hinaus auch die Nutzung durch die von ihm ausgewählte Vertrauensperson. Das ergibt sich im Umkehrschluss aus einer Kontrollüberlegung: Wären die Erben nicht an die Lizenz gebunden, weil sie nicht gleichzeitig die Accountberechtigung innehaben, liefe die Möglichkeit zur Benennung einer Vertrauensperson stets leer. Der Provider könnte in diesem Fall gegenüber der Vertrauensperson nie die zum Betrieb des Accounts erforderlichen Nutzungshandlungen durchführen. Dem Willen der Nutzungs- und Lizenzvertragsparteien - insbesondere dem Willen des Erblassers und Urhebers, der die Möglichkeit zur Benennung einer Vertrauensperson bewusst wahrgenommen hat - würde ein solches Ergebnis kaum gerecht. Im Gegenteil spricht der beschränkte Umfang der Nutzungsrechtseinräumung, der insbesondere keine gewerbliche Verwertung der Inhalte durch den Provider umfasst, dafür, dass den Erben eine fortgesetzte Bindung an die Lizenz zumutbar ist.

843 Nach Ziff. 3.3.1 der AGB von Facebook (s. Fn. 781) ist die Lizenz explizit auf die Löschung des Accounts auflösend bedingt; ähnlich auch die AGB von Instagram (s. Fn. 784); die AGB von Google (s. Fn. 827) sehen eine Befristung der Lizenz vor; vgl. zur Befristung und auflösenden Bedingung urheberrechtlicher Nutzungsrechte Berger in: Berger/Wündisch (Hrsg.), Urhebervertragsrecht, $\mathbb{1}$ Rn. $196 \mathrm{f}$. 


\section{Zwischenergebnis zu II.}

Zusammengefasst kann festgehalten werden, dass der Erblasser zu seinen Lebzeiten dem Provider in der Regel formularvertraglich die zum Plattformbetrieb technisch-funktional notwendigen Nutzungsrechte wirksam einräumt. Der Einräumung inhaltlich oder zeitlich weitergehender Rechte sind hingegen enge Grenzen gesetzt. Nach dem Erbfall sind die Erben als neue Urheberrechtsinhaber grundsätzlich an die dem Provider eingeräumte Lizenz gebunden. Das gilt solange und soweit der Account nicht gelöscht wird und unabhängig von der Frage, wer nach dem Willen des Erblassers ab dem Zeitpunkt des Erbfalls bezogen auf den Account zugangsund nutzungsberechtigt ist.

\section{Berechtigung an den Accountinhalten kraft Urheberrechts}

Im Regelfall gehen die nutzungsvertragliche Position der Accountinhaberschaft einerseits und die Urheber- und Leistungsschutzrechte an den Accountinhalten andererseits im Erbgang gemeinsam auf die Erben über. Ein anderes Ergebnis - also das Auseinanderfallen der Rechtspositionen - ist indes nicht ausgeschlossen. Die Accountinhaberschaft kann kraft nutzungsvertraglicher Vereinbarung mit dem Tod des Erblassers erlöschen oder statt auf die Erben auf eine Vertrauensperson übergehen. Die urheberrechtlichen Positionen können beispielsweise im Wege der Erfüllung eines Vermächtnisses auf einen vom Erblasser bestimmten Dritten oder an Miterben im Wege der Erbauseinandersetzung zu übertragen sein, vgl. \29 Abs. 1 UrhG. ${ }^{844}$

Vor diesem Hintergrund stellt sich die Frage, ob und inwieweit sich allein aus der Stellung als Urheberrechtsinhaber eine Berechtigung an den Accountinhalten ableiten lässt. Diese Berechtigung könnte sich durch ein Recht auf Löschung der Inhalte (dazu 1.) oder durch ein Recht auf Zugang zu ebenjenen (dazu 2.) ausdrücken. Vorweg kann festgehalten werden, dass sich etwaige Ansprüche des Urheberrechtsinhabers lediglich auf die urheber- beziehungsweise leistungsschutzrechtlich geschützten Anteile der

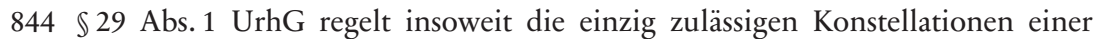
translativen Übertragung des Urheberrechts, vgl. dazu Obly in: Schricker/ Loewenheim (Hrsg.), $\$ 29$ UrhG Rn. 8 ff.; Hoche in: Wandtke/Bullinger (Hrsg.), UrhG, $\mathbb{} 29$ Rn. 24 ff.; Schulze in: Dreier/Schulze (Hrsg.), UrhG, $\mathbb{} 29$ Rn. 4 ff.; Schack, Urheber- und Urhebervertragsrecht, Rn. 648. 
Accountinhalte und daher (regelmäßig) weder auf den Account als solchen noch auf sämtliche Accountinhalte beziehen kann. ${ }^{845}$

\section{Recht des Urheberrechtsinhabers auf Löschung der Accountinhalte}

Der Urheberrechtsinhaber kann grundsätzlich mit Unterlassungs- und Schadensersatzansprüchen aus $\$ 97$ UrhG gegen die Verletzung seines Rechts vorgehen. ${ }^{846}$ Die Möglichkeit, Dritten auf diesem Wege den $\mathrm{Zu}-$ gang zu den Accountinhalten zu untersagen oder die Löschung der urheberrechtlich geschützten Teile des Accounts zu verlangen, findet indes eine wichtige Grenze in der dargestellten Bindung des Rechtsnachfolgers an die vom Urheber eingeräumte Lizenz. ${ }^{847}$ Zwar wird im Kontext nutzergenerierter Inhalte dem Rückruf von Nutzungsrechten wegen gewandelter Überzeugung gem. $\$ 42$ UrhG mitunter eine wichtige Bedeutung zugesprochen. ${ }^{848}$ Allerdings sieht $\$ 42$ Abs. 1 S. 2 UrhG für die Ausübung des Rückrufs durch den Rechtsnachfolger hohe Hürden vor. Außerdem ist bei der Ausübung urheberpersönlichkeitsrechtlicher Befugnisse, wie dargestellt, ${ }^{849}$ gerade das Interesse des Urhebers und nicht dasjenige seines Rechtsnachfolgers maßgeblich. ${ }^{850}$ Soweit der Erblasser das Auseinanderfallen von Account und Urheberrecht durch entsprechende Vorkehrungen selbst herbeigeführt hat, wird der nachgefolgte Urheberrechtsinhaber einen Löschungsanspruch zulasten des gewillkürten Accountinhabers kaum auf die Interessen des Erblassers stützen können.

Wenn und soweit der Provider oder die am Account berechtigte Vertrauensperson aber urheberrechtliche Nutzungshandlungen vornehmen,

$845 \mathrm{Zu}$ der Frage, an welchen Accountinhalten Urheber- bzw. Leistungsschutz bestehen kann, vgl. bereits S. $189 \mathrm{ff}$.

$846 \mathrm{Zu}$ den Anspruchsvoraussetzungen vgl. J.B. Nordemann in: Fromm/Nordemann, \$97 UrhG Rn. 8 ff.; Specht in: Dreier/Schulze (Hrsg.), UrhG, $\$ 97$ Rn. 3 ff.; ferner Meckel in: Dreyer/Kotthoff/Meckel/Hentsch (Hrsg.), $\$ 97$ UrhG Rn. $1 \mathrm{ff}$.

847 Vgl. dazu zuvor auf S. 205 f. Ein Nutzungsrecht lässt den Tatbestand aus $\$ 97$ Abs. 1 UrhG entfallen, s. Leistner in: Schricker/Loewenheim (Hrsg.), $\mathbb{9} 97$ UrhG Rn. 25.

848 So Rauda, GRUR 2010, 22, 22 ff.; sogar für freie Widerrufbarkeit Redeker in: Hoeren/Sieber/Holznagel (Hrsg.), MMR-HdB, Teil 12 Rn. 437 f.; a.A., wegen der Entschädigungspflicht gem. $\$ 42$ Abs. 3 UrhG werde die praktische Bedeutung des Rückrufs gering bleiben, Paul ebd., Teil 7.4 Rn. 141.

849 Siehe dazu Fn. 776.

850 Peukert in: Schricker/Loewenheim (Hrsg.), $\$ 42$ UrhG Rn.9 erklärt damit die „besonderen Voraussetzungen des [\$42] Abs. 1 S. 2"UrhG. 
die über den Umfang der eingeräumten Lizenz hinausgehen, zeitigt das Unterlassungsansprüche des Urheberrechtsinhabers, vgl. $\$ 97$ Abs. 1 UrhG. Mit Blick auf die inhaltlichen Beschränkungen der formularvertraglichen Lizenzierung ${ }^{851}$ ist damit insbesondere eine gewerbliche Verwertung der Inhalte jenseits der schlichten Nutzung des Accounts ausschließlich dem Urheberrechtsinhaber vorbehalten.

\section{Recht des Urheberrechtsinhabers auf Zugang zum Account}

Die Grundlage für einen Anspruch auf Accountzugang könnte $\$ 25$ Abs. 1 UrhG bilden, ${ }^{852}$ wonach der Urheberrechtsinhaber „vom Besitzer des Originals oder eines Vervielfältigungsstückes seines Werkes verlangen [kann], daß er ihm das Original oder das Vervielfältigungsstück zugänglich macht, soweit dies zur Herstellung von Vervielfältigungsstücken oder Bearbeitungen des Werkes erforderlich ist und nicht berechtigte Interessen des Besitzers entgegenstehen." Das Zugangsrecht schützt die Beziehung des Urhebers zu seinem Werk. ${ }^{853}$ Sollte der Urheberrechtsinhaber (vorübergehend) Zugang zu den Accountinhalten erhalten, könnte er unproblematisch ein Vervielfältigungsstück in Form einer digitalen Kopie herstellen. ${ }^{854}$ Allerdings muss der Anspruchssteller das Bezugsobjekt des Anspruchs aus $\$ 25$ Abs. 1 UrhG nach allgemeinen Grundsätzen benennen. ${ }^{855}$ Interesse dürfte aber gerade an denjenigen Inhalten bestehen, die nicht ohnehin öffentlich einsehbar sind und die dem Anspruchssteller daher weder bekannt sind noch von ihm konkret benannt werden können. ${ }^{856}$ Außerdem erfasst $\$ 25$ Abs. 1 UrhG jedenfalls nur die Teile des Accounts, die Schutz nach Maßgabe des UrhG genießen ${ }^{857}$ - mit der Folge, dass sich aus dem Urheberrecht

851 Vgl. dazu zuvor auf S. $198 \mathrm{ff}$.

852 So zuerst Brinkert/Stolze/Heidrich, ZD 2013, 153, 155.

853 Wiebe in: Spindler/Schuster (Hrsg.), Recht der elektronischen Medien, $\$ 25$ UrhG Rn. 1; Schulze in: Dreier/Schulze (Hrsg.), UrhG, $\mathbb{} 25$ Rn. 1 („unauflösliches urheberpersönlichkeitsrechtliches Band zwischen Urheber und Werk"); Bullinger in: Wandtke/Bullinger (Hrsg.), UrhG, $\mathbb{} 25$ Rn. 1.

854 Kutscher, Der digitale Nachlass, S. 38.

855 Auf diese Hürde hinweisend Kutscher, Der digitale Nachlass, S. 37 ff.; Fußeder, Soziale Netzwerke im Nachlass, S. 28; Thiesen, Daten in der Erbmasse, S. $152 \mathrm{f}$.

856 Ebd. (Fn. 855).

857 Das sind insbesondere Werke i.S.v. $\$ 2$ UrhG und Lichtbilder i.S.v. $\$ 72$ UrhG, Laufbilder i.S.v. $\$ 95$ UrhG sind hingegen nicht erfasst, vgl. Schulze in: Dreier/ Schulze (Hrsg.), UrhG, $\$ 25$ Rn. 8; Vogel in: Schricker/Loewenheim (Hrsg.), $\$ 25$ UrhG Rn. 8 a.E. 
kein vollständiger Zugang ableiten lässt, der mit der nutzungsvertraglichen Position vergleichbar wäre. ${ }^{858}$ Insbesondere alle von den Kommunikationspartnern des Erblassers empfangenen Nachrichten und Medien können hier kein Bezugsobjekt im Rahmen von $\$ 25$ Abs. 1 UrhG sein.

\section{Zwischenergebnis zu III.}

Aus dem Urheberrecht allein kann der Rechtsnachfolger kein Herrschaftsrecht über den Account und seine Inhalte ableiten. Hat der Erblasser Vorkehrungen getroffen, die in Abweichung vom gesetzlichen Regelfall dazu führen, dass die Account- und die Urheberrechtsinhaberschaft auseinanderfallen, dann dürfen die Konsequenzen dieser Entscheidung nicht über den Umweg des Urheberrechts nivelliert werden. Das gilt für den Anspruch des Urheberrechtsinhabers auf Löschung des Accounts, welcher an der fortlaufenden Wirksamkeit der Nutzungsrechte des Providers scheitert, ebenso wie für den entsprechenden Zugangsanspruch, für den bereits keine hinreichende Anspruchsgrundlage ersichtlich ist.

\section{Zwischenergebnis zu C.}

Die Urheberrechte des Erblassers können als eigenständiges Erbrechtsobjekt im digitalen Nachlass untersucht werden. Ein Nutzer sozialer Medien bringt zahlreiche Inhalte in seinen Account ein, die Urheberrechts- oder zumindest Leistungsschutz nach dem UrhG genießen. Die bestehenden Immaterialgüterrechte gehen im Erbfall auf die Erben über. Dieser Übergang schließt die an den Inhalten eingeräumten Nutzungsrechte des Providers ein, welche im Grundsatz auch den Rechtsnachfolger binden, soweit nichts anderes bestimmt ist oder aus dem Zweck der Nutzungsrechtseinräumung folgt. Der Zweck bildet insofern sowohl zu Lebzeiten des Nutzers als auch nach dem Erbfall die Grenze der (formularvertraglichen) Lizenzierung an den Provider. Das Urheberrecht vermittelt seinem Inhaber derweil keine Rechtsposition, welche die nutzungsvertragliche Accountinhaberschaft ersetzen kann.

858 Thiesen, Daten in der Erbmasse, S. 152 f.; ferner Fußeder, Soziale Netzwerke im Nachlass, S. 28 


\section{Schlussbetrachtungen zu Kapitel 3}

Die Untersuchung der Rechtsverhältnisse zwischen Provider und Nutzer als Erbrechtsobjekt(e) erfordert es, das sich dynamisch entwickelnde Angebot von Informations- und Kommunikationsdiensten sowie das damit zusammenhängende Nutzungsverhalten aus der Perspektive unterschiedlicher Rechtsmaterien zu beleuchten. Dabei besteht ein Interesse des Rechtsverkehrs an einer praktikablen Handhabung und klaren, möglichst einheitlichen Regeln zum Umgang mit dem digitalen Nachlass. In Übereinstimmung mit dem Facebook-Urteil des $\mathrm{BGH}^{859}$ ist von einem sukzessionsfreundlichen Regel-Ausnahme-Verhältnis auszugehen, wonach die Übergangsfähigkeit der nutzungsvertraglichen Position im Erbgang sowie die Durchsetzbarkeit des daraus abgeleiteten Rechts auf Zugang zu und Nutzung des Accounts den Regelfall und Ausgangspunkt bildet. Insofern ist auch keine Kollision zwischen der nutzungsvertraglichen und der urheberrechtlichen Erbrechtsposition feststellbar.

Allerdings scheint der in der Debatte vielfach bemühte Vergleich zu analogen Nachlassgegenständen, wie Briefpost oder einem Tagebuch, Ausdruck des Wunsches danach zu sein, Nutzerkonten als - wenn auch neuartigen - Erbrechtsobjekten keinerlei Sonderstatuts im überkommenen Regelungsregime des Erbrechts zuzubilligen. Indessen darf die Komplexität der aufgeworfenen Fragen nicht im Interesse des Rechtsverkehrs negiert werden. Denn Nutzerkonten treten im Informationszeitalter nicht schlicht an die Stelle eines herkömmlichen Briefwechsels. Vielmehr gehen sie darüber hinsichtlich ihrer Funktion, ihres Umfangs und ihrer Vielfalt weit hinaus. Aus der Eigenschaft von Nutzerkonten als digitalem Abbild unterschiedlichster Lebensbereiche resultiert eine Vielschichtigkeit, der auch juristisch Rechnung zu tragen ist. Vor diesem Hintergrund sollte dem Drängen auf eine vollständige Gleichsetzung von Analogem und Digitalem nicht voreilig nachgegeben werden. Stattdessen sollten gerade diejenigen Regelungsregime fruchtbar gemacht werden, die eine einzelfallgerechte Differenzierung zwischen unterschiedlich gelagerten Sachverhalten ermöglichen. Das sind namentlich das unionale Datenschutzrecht sowie der vertragliche Gestaltungsspielraum von Provider und Nutzer. ${ }^{860}$ Schon gegenwärtig ist es rechtlich geboten und inhaltlich sachgemäß, zu unterscheiden beispiels-

859 BGH NJW 2018, 3178.

$860 \mathrm{Zu}$ Vertragsabreden betreffend die Vererbbarkeit s. S. $159 \mathrm{ff}$. Zur Einräumung urheberrechtlicher Nutzungsrechte an den Accountinhalten des Erblassers s. S. $194 \mathrm{ff}$. 
weise zwischen unterschiedlichen Internetdiensten, ${ }^{861}$ zwischen gewerblich und privat genutzten Accounts, ${ }^{862}$ zwischen einfachen und sensiblen personenbezogenen Daten ${ }^{863}$ oder zwischen minder- und volljährigen Nutzern. ${ }^{864}$ Sollte das vielfältige Angebot von Informations- und Kommunikationsdiensten erwartungsgemäß seine Expansion in verschiedenste Aspekte unserer Lebensführung fortsetzen, wird die Notwendigkeit eines differenzierenden und einzelfallorientierten Vorgehens weiter zunehmen. Nur so können die kollidierenden Interessen in der multipolaren Konstellation des digitalen Nachlasses zu einem schonenden Ausgleich gebracht werden.

861 Vgl. dazu die Ausführungen zum vertragsimmanenten Schutz der Kommunikationspartner des Erblassers auf S. $94 \mathrm{ff}$.

862 Vgl. dazu etwa die Ausführungen zum IPR auf S. $74 \mathrm{ff}$. oder zum UWG auf S. $104 \mathrm{f}$.

863 Vgl. dazu die Ausführungen zur Eröffnung des Anwendungsbereichs von Art. 9 DS-GVO auf S. $142 \mathrm{ff}$.

864 Vgl. dazu die Ausführungen zu Art. 6 Abs. 1 S. 1 lit. f) a.E. DS-GVO auf S. 137 ff. 


\section{Kapitel 4 - Fazit und Ausblick}

Mit Urteil vom 12. Juli 2018 hat der BGH entschieden, dass der Nutzungsvertrag zwischen dem Anbieter eines sozialen Netzwerks und dem jeweiligen Accountinhaber bei dessen Tod gem. $\ 1922$ Abs. 1 BGB auf die Erben übergeht. ${ }^{865}$ Soweit an dieser sukzessionsfreundlichen Entscheidung zur Vererbbarkeit eines Facebook-Accounts Kritik geäußert wird, stehen dahinter schwerpunktmäßig grundsätzliche Bedenken des Daten- und Persönlichkeitsschutzes. ${ }^{866}$ Die Digitalisierung ermöglicht es in einem stetig wachsenden Maße, jedes Detail der persönlichen Lebensführung aufzuzeichnen und bis weit über den Tod aller Beteiligten hinaus zu archivieren. Damit gehen unterschiedliche Fragen nach der Grundlage und den Grenzen dieser Form der Datenverarbeitung sowie Debatten über Prävention und Sensibilität der Nutzer einher. Wie aktuell dieses übergeordnete Thema ist, zeigen beispielsweise die Diskussion um das sogenannte Recht auf Vergessenwerden ${ }^{867}$ oder der Vorschlag eines „Verfallsdatums von Daten" ${ }^{868}$. Dass der Daten- und Persönlichkeitsschutz auch und gerade im Zusammenhang mit dem digitalen Nachlass in diesem Sinne besondere Aufmerksamkeit erfährt, ist nicht überraschend. Denn nicht zuletzt ist der Tod des Nutzers eine Zäsur, die allgemein zum Nachdenken über den Untergang beziehungsweise den Fortbestand von Rechtspositionen anregt, aus denen sich der Zugang zu mitunter sensiblen Informationen ergibt. Zwar ist der sukzessionsfreundlichen Linie zuzugestehen, dass im Zusammenhang mit dem Erbfall nicht gleichsam inzidenter die (rechts-)politische Debatte um einen möglichen postmortalen Datenschutz geführt und

865 BGH NJW 2018, 3178, Ls. (Dem Zugang der Erben zu dem Account nebst darin gespeicherten Inhalten stehe „weder das postmortale Persönlichkeitsrecht des Erblassers noch das Fernmeldegeheimnis oder das Datenschutzrecht entgegen.").

866 Das gilt insbesondere für Martini/Kienle, JZ 2019, 235. Aus der vorliegenden Arbeit s. insofern etwa die Ausführungen zu Art. 9 DS-GVO (S. 142 ff.) oder zum vertragsimmanenten Schutz der Kommunikationspartner des Erblassers (S. 94 ff.).

$867 \mathrm{Zu}$ Entstehungsgeschichte und Telos von Art. 17 DS-GVO, den die DS-GVO selbst als „Recht auf Vergessenwerden“ bezeichnet, s. Paal in: Paal/Pauly (Hrsg.), DS-GVO, Art. 17 Rn. 2 ff. m.w.N.

868 Budzikiewicz zitiert nach dem Diskussionsbericht von Bornhauser, AcP (218) 2018, 594, 596; Mayer-Schönberger, Delete, S. $201 \mathrm{ff}$. 
die (vermeintlich) mangelnde digitale Kompetenz und Sorgfalt der Nutzer kompensiert werden kann. ${ }^{869}$ Indessen genießt das deutsche Erbrecht keinen Vorrang vor anderen Normenregimen wie etwa dem Datenschutzrecht der DS-GVO. Denn dem Sukzessionsgedanken aus $\mathbb{1 9 2 2}$ Abs. 1 BGB kommt selbst in Ansehung seiner verfassungsrechtlichen Verankerung in der Erbrechtsgarantie aus Art. 14 Abs. 1 S. 1 Var. 2 GG kein absoluter Geltungsanspruch zu. Vielmehr sind etwaige Ziel- und Interessenkonflikte mittels der klassischen rechtswissenschaftlichen Methodik möglichst schonend aufzulösen. Auf Grundlage der Auslegung des geltenden Rechts ergeben sich daraus folgende Thesen der vorliegenden Arbeit.

\section{Zusammenfassung in Thesen}

1. Zur Bestimmung des Erbrechtsobjekts im Verhältnis zwischen dem Anbieter von Informations- und Kommunikationsdiensten und dem Nutzer ist zwischen dem Nutzungsvertrag einerseits und etwaigen Urheber- und Leistungsschutzrechten nach Maßgabe des UrhG an den Accountinhalten andererseits zu unterscheiden. Bei dem Nutzungsvertrag handelt es sich in der Regel um einen Vertrag sui generis, der für den Nutzer unentgeltlich ist und je nach genauer Ausgestaltung des Internetdienstes dienst-, werkund/oder mietvertragliche Leistungspflichten des Providers vorsieht. ${ }^{870}$

2. De lege lata existiert kein einheitliches, eigentumsähnliches Recht an Daten als solchen. Damit scheiden sowohl eine Verdinglichung des Erbrechtsobjekts als auch insgesamt eine Vereinheitlichung des digitalen Nachlasses aus. Die rechtspolitische Diskussion und ein etwaiges Tätigwerden des (Unions-)Gesetzgebers gilt es indes aufmerksam zu verfolgen, weil eine Entscheidung über das Zuordnungsregime für Daten auf die Bestimmung des Erbrechtsobjekts (auch unbeabsichtigt) zurückwirken kann. ${ }^{871}$

3. Internationalprivatrechtlich lässt sich keine allgemeingültige Anknüpfung für Rechtsfragen des digitalen Nachlasses benennen. Denn die Vererbbarkeit einer Rechtsposition richtet sich nicht nach dem Erbstatut, sondern nach dem jeweiligen Statut des betroffenen Rechtsverhältnisses. Da-

869 Herzog, AnwBl Online 2018, 472, 474 („[...] wir müssen schon lebzeitig einen verantwortungsvollen Umgang mit den neuen Medien erlernen und für den Fall der Geschäftsunfähigkeit und des Todesfalls auch insoweit Vorsorge treffen." ${ }^{\prime \prime}$.

$870 \mathrm{Zu}$ These Nr. 1 s. S. $31 \mathrm{ff}$.

$871 \mathrm{Zu}$ These Nr. 2 s. S. $44 \mathrm{ff}$. 
mit wirkt die rechtstatsächliche Vielfalt des digitalen Nachlasses in das Kollisionsrecht fort. Nutzungsverträge sind vielfach Verbraucherverträge und daher nach den Sonderregeln des Art. 6 Abs. 1 und 2 Rom I-VO anzuknüpfen. Bei Nutzungsverträgen ohne Verbraucherbeteiligung kommt hingegen der Rechtswahl nach Art. 3 Rom I-VO und der objektiven Grundanknüpfung nach Art. 4 Rom I-VO entscheidende Bedeutung zu. Das Phänomen des sog. Influencings mittels reichweitestarker Accounts kann hier Abgrenzungsschwierigkeiten in inhaltlicher wie zeitlicher Hinsicht auslösen. 872

4. Nach allgemeinen Grundsätzen geht die nutzungsvertragliche Position des Erblassers mit dem Erbfall gem. $\$ 1922$ Abs. 1 BGB auf die Erben über. Das gilt in Anlehnung an den Rechtsgedanken des $\$ 399$ Fall 1 BGB nur dann nicht, wenn das Vertragsverhältnis entscheidend von höchstpersönlichen Zwecken geprägt ist und durch eine Rechtsnachfolge in seinem Wesen verändert würde. Das Merkmal der Höchstpersönlichkeit steht der Übergangsfähigkeit im Verhältnis zwischen dem Erblasser und seinen eigenen Erben nicht entgegen. Im Verhältnis zwischen dem Provider, dem Erblasser und dessen Kommunikationspartnern sind hingegen die vernünftigen Erwartungen der Nutzer an die Übergangsfähigkeit und hinsichtlich der Verfügungsbefugnis an Accountinhalten nach dem Absenden für den jeweiligen Internetdienst zu prüfen. Die Vererbbarkeit ist insofern zwar die Regel, nicht aber das zwingende Ergebnis. ${ }^{873}$

5. Wenn und soweit ein Nutzungsvertrag vererbbar ist, schließt das Erbrecht neben dem Recht auf Accountzugang auch die aktive Weiternutzung des übergegangenen Nutzungskontos ein. Eine etwaige (beispielsweise lauterkeitsrechtliche) Beschränkung des Rechts auf aktive Nutzung berührt die Übergangsfähigkeit dieses Rechts nicht. Aus der Rechtsprechung des BGH zur Vererbbarkeit eines Girokontos folgt kein durchschlagendes Argument gegen dieses Ergebnis. Der Vergleich zu Girokonten sollte in der Debatte um den digitalen Nachlass insgesamt nicht länger als Argumentationshilfe herangezogen werden. ${ }^{874}$

6. Die Zugangsgewährung durch die Provider gegenüber den Erben fällt in den Anwendungsbereich der DS-GVO. Nicht der Erblasser, aber dessen Kommunikationspartner sind betroffene Personen i.S.v. von Art. 4 Nr. 1 DS-GVO. Die Zugangsgewährung steht damit unter dem Grundverordnungsregime und unterfällt grundsätzlich dem präventiven Verbot mit Er-

$872 \mathrm{Zu}$ These Nr. 3 s. S. $70 \mathrm{ff}$.

$873 \mathrm{Zu}$ These Nr. 4 s. S. $87 \mathrm{ff}$.

$874 \mathrm{Zu}$ These Nr. 5 s. S. $100 \mathrm{ff}$. 
laubnisvorbehalt des Art. 6 Abs. 1 S. 1 DS-GVO. Der nutzungsvertragliche Zugangsanspruch der Erben ist in der Folge nur soweit durchsetzbar, wie seine Erfüllung durch den Provider mit der DS-GVO in Einklang steht. ${ }^{875}$

7. Bei allen in Betracht kommenden Erlaubnistatbeständen aus Art. 6 Abs. 1 DS-GVO sind die Erwartungen der Kommunikationspartner an die Übergangsfähigkeit (s. bereits These 4) von wesentlicher Bedeutung. Wird die Zugangsgewährung auf die Interessenabwägung nach lit. f) gestützt, sind am Maßstab des Tatbestandsmerkmals der Erforderlichkeit mögliche Alternativen zum (umfassenden) Zugang der Erben im Einzelfall zu prüfen. Ein Mittel zur sachgemäßen Differenzierung zwischen unterschiedlichen Betroffenen kann sich außerdem aus dem Widerspruchsrecht des Betroffenen gem. Art. 21 Abs. 1 S. 1 DS-GVO ergeben. ${ }^{876}$

8. Umfasst der Erblasseraccount sensible Daten der Kommunikationspartner i.S.v. Art. 9 DS-GVO, richtet sich die datenschutzrechtliche Zulässigkeit der Zugangsgewährung allein nach dem im Verhältnis zu Art. 6 DS-GVO spezielleren Art. 9 DS-GVO. Aus der intensiven Nutzung von Internetdiensten durch viele Privatpersonen und dem breit formulierten Wortlaut des Anwendungsbereichs („hervorgehen“) folgt, dass Art.9 DSGVO nicht selten eröffnet sein wird. In diesem Fall begegnet die Zugangsgewährung gegenüber den Erben erheblichen Schwierigkeiten, weil der Katalog von Ausnahmetatbeständen aus Art. 9 Abs. 2 DS-GVO keinen allgemeinen Abwägungstatbestand enthält. ${ }^{877}$

9. Der Provider ist gem. $\$ 7$ Abs. 3 S. 2 TMG zur Wahrung des Fernmeldegeheimnisses aus $\$ 88$ TKG verpflichtet. Dass der BGH zur Vermeidung einer absoluten Zugangshürde annimmt, die Erben seien keine „anderen“ i.S.v. $\$ 88$ Abs. 3 S. 1 TKG, bewegt sich als ergebnisgetriebene Argumentation an den Grenzen der Auslegung. Den pragmatischen Ansatz des BGH sollte der Gesetzgeber im Zuge der ohnehin aufgrund neuer unionaler Vorgaben notwendig gewordenen TKG-Novelle durch eine Auflösung der Normkollision von Fernmeldegeheimnis und Erbrecht (weiter) festigen. Den Interessen der Kommunikationspartner, die $\mathbb{} 88$ TKG hier schützen soll, wird bereits durch die DS-GVO hinreichend Rechnung getragen. ${ }^{878}$

10. Kraft ihrer Privatautonomie können die Vertragsparteien von dem gesetzlichen Ergebnis der Vererbbarkeit eines Nutzerkontos abweichen. Diesem Gestaltungsspielraum sind indessen praktische und rechtliche

$875 \mathrm{Zu}$ These Nr. 6 s. S. $119 \mathrm{ff}$.

$876 \mathrm{Zu}$ These Nr. 7 s. S. $124 \mathrm{ff}$.

$877 \mathrm{Zu}$ These Nr. 8 s. S. $142 \mathrm{ff}$.

$878 \mathrm{Zu}$ These Nr. 9 s. S. $148 \mathrm{ff}$. 
Grenzen gesetzt. Insbesondere darf der Provider die Gestaltungs- und Verfügungsbefugnis des Erblassers über seinen Nachlass nicht einseitig mittels AGB beschränken. Dagegen ist das Interesse der Erben an der Rechtsnachfolge für die Beurteilung der Wirksamkeit nutzungsvertraglicher Bestimmungen unbeachtlich. Unabhängig von Form und Inhalt der Vereinbarung bleibt der Provider umfassend an seine Pflichten nach Maßgabe der DS-GVO gebunden, die er gegenüber den Kommunikationspartnern des Erblassers trägt. Diese Pflichten sind im Verhältnis zwischen Erblasser und Anbieter nicht abdingbar. ${ }^{879}$

11. An einer Vielzahl, nicht aber an sämtlichen Inhalten, die der Erblasser lebzeitig in seinen Account einbringt, besteht Urheberrechts- oder zumindest Leistungsschutz nach dem UrhG. Die Immaterialgüterrechte gehen im Erbfall vollumfänglich auf die Erben über, $\mathbb{} 28$ Abs. 1 UrhG. Dasselbe gilt spiegelbildlich für die an den Inhalten eingeräumten Nutzungsrechte des Providers, welche grundsätzlich auch den Rechtsnachfolger binden, soweit nichts anderes vereinbart ist oder aus dem Zweck der Nutzungsrechtseinräumung folgt. Der Zweck stellt damit erst zu Lebzeiten des Nutzers und sodann im Erbfall die Grenze der (formularvertraglichen) Lizenzierung an den Anbieter dar. Die immaterialgüterrechtliche Position vermittelt ihrem Inhaber derweil keine ersatzweise Berechtigung am Account, die von der nutzungsvertraglichen Position unabhängig wäre. ${ }^{880}$

\section{Ausblick}

Während die Regierungsparteien in dem am 12. März 2018 unterzeichneten Koalitionsvertrag die Absicht festgehalten hatten, „die Vererbbarkeit des digitalen Eigentums (z.B. Nutzer Accounts, Datenbestände) rechtssicher gesetzlich [zu] regeln" ${ }^{881}$, sieht die Bundesregierung im Anschluss an das Facebook-Urteil des BGH vom 12. Juli 2018 aktuell keinen Regelungsbedarf (mehr). ${ }^{882}$ Es bleibt abzuwarten, ob der Gesetzgeber dauerhaft da-

$879 \mathrm{Zu}$ These Nr. 10 s. S. $159 \mathrm{ff}$.

$880 \mathrm{Zu}$ These Nr. 11 s. S. $186 \mathrm{ff}$.

881 Ein neuer Aufbruch für Europa. Eine neue Dynamik für Deutschland. Ein neuer Zusammenhalt für unser Land. Koalitionsvertrag zwischen CDU, CSU und SPD, Zeile $6175 \mathrm{f}$.

882 In BT-Drs. 19/4207, S. 2 heißt es: „Die Bundesregierung sieht insoweit keinen Handlungsbedarf im Erbrecht." Hierbei handelt es sich um eine Antwort auf eine kleine Anfrage der FDP-Bundestagsfraktion, vgl. BT-Drs. 19/3954. 
von absieht, explizite Regelungen zum digitalen Nachlass zu erlassen. ${ }^{883}$ Ungeachtet dessen steht zu erwarten, dass andere rechtspolitische Diskussionen und Gesetzgebungsprojekte im Bereich des Rechts der Informationsgesellschaft auf die in dieser Arbeit aufgeworfenen Fragen (unbeabsichtigt) zurückwirken werden. ${ }^{884} \mathrm{Zu}$ dieser potenziell dynamischen Rechtslage kommen der technische Fortschritt beim Angebot der Provider und die damit in Zusammenhang stehende Entwicklung des Nutzungsverhaltens der User. Beides kann sich auf die einzelfallspezifische Beurteilung auswirken, die insbesondere bei der Prüfung möglicher datenschutzrechtlicher Beschränkungen der auf die Erben übergegangenen Rechtsposition vorzunehmen ist. Zusammen mit der prognostizierten Zunahme von Erbfällen, die (auch) einen digitalen Nachlass zur Folge haben, ${ }^{885}$ ergibt sich aus alledem gleichsam ein in Bewegung befindliches Kaleidoskop denkbarer rechtlicher Konflikte, welches sich pauschalen Lösungsansätzen verschließt. Demnach werden Literatur und Rechtsprechung weiterhin gefordert sein, die bestehenden und zukünftig auftretenden Aspekte des digitalen Nachlasses einer sachgerechten Lösung zuzuführen und allfällige Interessenskollisionen möglichst schonend aufzulösen. Vor diesem Hintergrund möchte die vorliegende Arbeit zu einer fortgesetzten Auseinandersetzung mit dem digitalen Nachlass einladen, deren Ausgangs-, nicht aber Schlusspunkt im Urteil des BGH zur Vererbbarkeit eines Facebook-Kontos zu sehen ist.

883 Dies bleibt mit Blick auf eine mögliche Veränderung unter den Regierungsparteien nach den Bundestagswahlen $2021 \mathrm{zu}$ beobachten. So forderte etwa die FDP-Bundestagsfraktion jüngst legislatives Handeln, vgl. BT-Drs. 19/14044, dazu Lieder/Berneith, ZRP 2020, 87; zur Aufforderung der Datenethikkommission, auch nach dem Facebook-Urteil des BGH die Normkollision zwischen Fernmeldegeheimnis und Erbrecht legislativ aufzulösen, vgl. bereits den Nachweis in Fn. 606.

884 Genannt seien hier etwa das Recht an Daten, s. dazu S. 66 f., oder die anstehende TKG-Novelle, s. dazu S. 158.

885 S. hierzu bereits die Nachweise in Fn. 11 und 12. 


\section{Literaturverzeichnis}

Ablberg, Hartwig/Götting, Horst-Peter, Beck'scher Online-Kommentar Urheberrecht, 29. Edition, München September 2020 (zitiert als Bearbeiter in: BeckOK Urheberrecht).

Albrecht, Jan Philipp/Jotzo, Florian, Das neue Datenschutzrecht der EU. Grundlagen. Gesetzgebungsverfahren. Synopse, Baden-Baden 2017 (zitiert als Albrecht/Jotzo, Das neue Datenschutzrecht der EU).

Alexander, Christian, Digitaler Nachlass als Rechtsproblem? Überlegungen aus persönlichkeitsrechtlicher, datenschutzrechtlicher und vertragsrechtlicher Sicht, K\&R 2016, S. 301-307.

ders., Anmerkung zu KG Berlin, Urteil v. 31.05.2017 - 21 U 9/16, notar 2017, S. 355-358.

ders., Anmerkung zu BGH, Urteil v. 12.07.2018 - III ZR 183/17, notar 2018, S. 367-368.

Amstutz, Marc, Dateneigentum. Funktion und Form, AcP 218 (2018), S. 439-551.

Amend-Traut, Anja/Hergenröder, Cyril H., Kryptowährungen im Erbrecht, ZEV 2019, S. 113-121.

Ann, Christoph/Kroiß, Ludwig/Mayer, Jörg, Nomos Kommentar. BGB. Erbrecht. Band 5: S\$1922-2385, 5. Auflage, Baden-Baden 2018 (zitiert als Bearbeiter in: NK-BGB).

Apel, Simon, Anmerkung zu BGH, Urteil v. 12.7.2018 - III ZR 183/17, ZD 2018, S. 486-488.

Arndt, Hans-Wolfgang/Fetzer, Thomas/Scherer, Joachim/Graulich, Kurt, TKG. Telekommunikationsgesetz. Kommentar, 2. Auflage, Berlin 2015 (zit. als Bearbeiter in: Arndt/Fetzer/Scherer/Graulich (Hrsg.), TKG).

Auer-Reinsdorff, Astrid/Conrad, Isabell, Handbuch IT- und Datenschutzrecht, 3. Auflage, München 2019 (zitiert als Bearbeiter in: Auer-Reinsdorff/Conrad (Hrsg.), Handbuch IT- und Datenschutzrecht).

Bar, Christian v./Mankowski, Peter, Internationales Privatrecht. Band II. Besonderer Teil, 2. Auflage, München 2019 (zitiert als $v$. Bar/Mankowski, Internationales Privatrecht II).

Bauer, Christian Alexander, User Generated Content. Urheberrechtliche Zulässigkeit nutzergenerierter Medieninhalte, Heidelberg u.a. 2011 (zitiert als: Bauer, User Generated Content). 
ders., User Generated Content - Urheberrechtliche Zulässigkeit nutzergenerierter Medieninhalte, in: Große Ruse-Khan, Henning/Klass, Nadine/v. Lewinski, Silke (Hrsg.), Nutzergenerierte Inhalte als Gegenstand des Privatrechts. Aktuelle Probleme des Web 2.0, Berlin, Heidelberg 2010, S. 1-42 (zitiert als Bauer, User Generated Content, in: in: Große Ruse-Khan/Klass/v. Lewinski (Hrsg.), Nutzergenerierte Inhalte als Gegenstand des Privatrechts).

Becker, Maximilian, Lauterkeitsrechtlicher Leistungsschutz für Daten, GRUR 2017, S. 346-355.

Berberich, Matthias, Virtuelles Eigentum, Tübingen 2010.

ders., Der Content „gehört” nicht Facebook! AGB-Kontrolle der Rechteeinräumung an nutzergenerierten Inhalten, MMR 2010, S. 736-741.

ders., Virtuelles Eigentum - Der Dualismus von Rechten am Werk und am Werkstück in der digitalen Welt, in: Große Ruse-Khan, Henning/Klass, Nadine/v. Lewinski, Silke (Hrsg.), Nutzergenerierte Inhalte als Gegenstand des Privatrechts. Aktuelle Probleme des Web 2.0, Berlin, Heidelberg 2010, S. 165-206 (zitiert als Berberich, Virtuelles Eigentum in: Große Ruse-Khan/Klass/v. Lewinski (Hrsg.), Nutzergenerierte Inhalte als Gegenstand des Privatrechts).

ders., Absolute Rechte an der Nutzung einer Domain - eine zentrale Weichenstellung für die Rechtsentwicklung, WRP 2011, S. 543-549.

ders., Zum Leitbildcharakter urheberrechtlicher Rechtsgrundsätze. Zugleich Besprechung von BGH, Urteil v. 31.05.2012 - I ZR 73/10 - Honorarbedingungen Freie Journalisten, WRP 2012, S. 1055-1059.

ders./Golla, Sebastian, Zur Konstruktion eines „Dateneigentums“ - Herleitung, Schutzrichtung, Abgrenzung, PinG 2016, S. 165-176.

Berger, Christian/Wündisch, Sebastian, Urhebervertragsrecht. Handbuch, 2. Auflage, Baden-Baden 2015 (zitiert als Bearbeiter in: Berger/Wündisch (Hrsg.), Urhebervertragsrecht).

Biermann, Bastian, Der digitale Nachlass im Spannungsfeld zwischen Erbrecht und Datenschutz. Zugleich Besprechung des Urteils des KG Berlin vom 31. Mai 2017 - 21 U 9/16, ZErb 2017, S. 210-217.

ders., Anmerkung zu BGH, Urteil v. 12.7.2018 - III ZR 183/17, ErbR 2018, S. 577-579.

Bisges, Marcel, Die Kleine Münze im Urheberrecht. Analyse des ökonomischen Aspekts des Werkbegriffs, Baden-Baden 2014 (zitiert als Bisges, Die Kleine Münze im Urheberrecht).

ders., Die Kleine Münze, der Dreigroschenprozess und der Herstellungsaufwand, GRUR 2015, S. 540-546.

ders., Handbuch Urheberrecht, Berlin 2016 (zitiert als Bearbeiter in: Bisges (Hrsg.), Handbuch Urheberrecht).

Boehm, Franziska, Herausforderungen von Cloud Computing-Verträgen: Vertragstypologische Einordnung, Haftung und Eigentum an Daten, ZEuP 2016, S. 358-387.

Bock, Merle, Juristische Implikationen des digitalen Nachlasses, AcP (217) 2017, S. 370-417. 
Bornhauser, Philip, Diskussionsbericht zum Referat von Christine Budzikiewicz, $\operatorname{AcP}(218)$ 2018, S. 594-600.

Bräutigam, Peter, Das Nutzungsverhältnis bei sozialen Netzwerken. Zivilrechtlicher Austausch von IT-Leistung gegen personenbezogene Daten, MMR 2012, S. 635-641.

Bräutigam, Peter/Herzog, Stephanie/Mayen, Thomas/Redeker, Helmut/Zuck, Holger, Stellungnahme des Deutschen Anwaltvereins durch die Ausschüsse Erbrecht, Informationsrecht und Verfassungsrecht zum Digitalen Nachlass. Stellungnahme Nr.: 34/2013, 2013 (zitiert als Bearbeiter in: DAV-Stellungnahme 34/2013).

Brinkert, Maike/Stolze, Michael/Heidrich, Joerg, Der Tod und das soziale Netzwerk. Digitaler Nachlass in Theorie und Praxis, ZD 2013, S. 153-157.

Brisch, Klaus/Müller-ter Jung, Marco, Digitaler Nachlass - Das Schicksal von E-Mailund De-Mail-Accounts sowie Mediencenter Inhalten, CR 2013, S. 446-455.

Britz, Thomas/Indenhuck, Moritz, Die Daten der Dritten - Verarbeitung drittbezogener Daten im Vertragsverhältnis, in: Taeger, Jürgen (Hrsg.), Rechtsfragen digitaler Transformationen - Gestaltung digitaler Veränderungsprozesse durch Recht, Tagungsband DSRI-Herbstakademie 2018, Edewecht 2018, S. 231-245 (zitiert als Britz/Indenhuck, Die Daten der Dritten - Verarbeitung drittbezogener Daten im Vertragsverhältnis, in: Taeger (Hrsg.), Tagungsband DSRI-Herbstakademie 2018).

Brox, Hans/Walker, Wolf-Dietrich, Allgemeines Schuldrecht, 44. Auflage, München 2020.

Bulowski, Stefan, Regulierung von Internetkommunikationsdiensten. Zur Anwendbarkeit des Telekommunikationsrechts auf Voice over IP, Instant Messaging und E-Mail-Dienste, Baden-Baden 2019 (zitiert als Bulowski, Regulierung von Internetkommunikationsdiensten).

Buchner, Benedikt/Kühling, Jürgen, Die Einwilligung in der Datenschutzordnung 2018, DuD 2017, S. 544-548.

Budzikiewicz, Christine, Digitaler Nachlass, AcP (218) 2018, S. 558-600.

Burandt, Wolfgang/Rojahn, Dieter, Erbrecht. BGB, FamFG, ZPO, BeurkG, GBO, EGBGB, EStG, ErbStG, EuErbVO. Kommentar, 3. Auflage, München 2019 (zitiert als Bearbeiter in: Burandt/Rojahn (Hrsg.)).

Burda, Max, Die Zweckbindung im Urhebervertragsrecht. $\$ 31$ Abs. 5 UrhG im Wandel der Zeit, Tübingen 2020 (zitiert als Burda, Die Zweckbindung im Urhebervertragsrecht).

Calliess, Christian/Ruffert, Matthias, EUV/AEUV, Das Verfassungsrecht der Europäischen Union mit Europäischer Grundrechtecharta, Kommentar, 5. Auflage, München 2016 (zitiert als Bearbeiter in: Calliess/Ruffert (Hrsg.), EUV/ AEUV).

Chrocziel, Peter, Einführung in den Gewerblichen Rechtsschutz und das Urheberrecht, 3. Auflage, München 2019.

Clément, Christoph, Urheberrecht und Erbrecht, Baden-Baden 1993. 
Culmsee, Thorsten, Postmortaler Datenschutz und postmortale Datennutzung, in: Taeger, Jürgen (Hrsg.), Law as a Service - Recht im Internet- und Cloud-Zeitalter, Tagungsband DSRI-Herbstakademie 2013, Edewecht 2013, S. 413-428 (zitiert als Culmsee, Postmortaler Datenschutz in: Taeger (Hrsg.), Tagungsband DSRI-Herbstakademie 2013).

Dann, Matthias/Markgraf, Jochen W., Das neue Gesetz zum Schutz von Geschäftsgeheimnissen, NJW 2019, S. 1774-1779.

Determann, Lothar, Gegen Eigentumsrechte an Daten. Warum Gedanken und andere Informationen frei sind und es bleiben sollten, ZD 2018, S. 503-508.

Deusch, Florian, Digitales Sterben - Das Erbe im Web 2.0, in: Taeger, Jürgen (Hrsg.), Law as a Service - Recht im Internet- und Cloud-Zeitalter, Tagungsband DSRI-Herbstakademie 2013, Edewecht 2013, S. 429-445 (zitiert als Deusch, Digitales Sterben - Das Erbe im Web 2.0, in: Taeger (Hrsg.), Tagungsband DSRI-Herbstakademie 2013).

ders., Digitales Sterben: Das Erbe im Web 2.0, ZEV 2014, S. 2-8.

ders., Der digitale Nachlass vor dem BGH und die Praxisfolgen, ZEV 2018, S. 687-691.

ders., Anmerkung zu KG Berlin, Beschluss v. 3.12.2019 - 21 W 11/19, ZEV 2020, S. 179-180.

Dietzel, Andreas, Untergang statt Fortbestand - Zur Abgrenzung der unvererblichen Rechtsbeziehungen im Schuldrecht, Pfaffenweiler 1992 (zitiert als Dietzel, Untergang statt Fortbestand).

Dix, Alexander, Daten als Bezahlung - Zum Verhältnis zwischen Zivilrecht und Datenschutzrecht, ZEuP 2017, S. 1-5.

Dopatka, Karsten, Forum. Digitaler Nachlass - Der Umgang mit elektronischen Daten nach dem Tod, NJW-aktuell 49/2010, S. 14-16.

Dorner, Michael, Umfassende Nutzungsrechteeinräumung gegen Pauschalabgeltung - Ende für „Buy-outs”? Aktuelle Entwicklungen der urhebervertragsrechtlichen Rechtsprechung und ihre Relevanz für die IT-rechtliche Vertragspraxis, MMR 2011, S. 780-785.

ders., Big Data und „Dateneigentum“. Grundfragen des modernen Daten- und Informationshandels, CR 2014, S. 617-628.

Dreier, Horst, Grundgesetz. Kommentar. Band I. Präambel, Artikel 1-19, 3. Auflage, Tübingen 2013 (zitiert als Bearbeiter in: Dreier (Hrsg.), GG).

Dreier, Thomas/Schulze, Gernot, Urheberrechtsgesetz. Verwertungsgesellschaftengesetz. Kunsturhebergesetz. Kommentar, 6. Auflage, München 2018 (zitiert als Bearbeiter in: Dreier/Schulze (Hrsg.), UrhG).

Dreyer, Gunda/Kotthoff, Jost/Meckel, Astrid/Hentsch Christian-Henner, Urheberrecht. Urheberrechtsgesetz. Verwertungsgesellschaftengesetz. Kunsturhebergesetz, 4. Auflage, Heidelberg 2018 (zitiert als Bearbeiter in: Dreyer/Kotthoff/Meckel/ Hentsch (Hrsg.), Urheberrecht). 
Dutta, Anatol/Weber, Johannes, Internationales Erbrecht. EuErbVO. Erbrechtliche Staatsverträge. EGBGB. IntErbRVG, München 2016 (zitiert als Bearbeiter in: Dutta/Weber, Internationales Erbrecht).

Ehmann, Eugen/Selmayr, Martin, Datenschutz-Grundverordnung. Kommentar, 2. Auflage, München 2018 (zitiert als Bearbeiter in: Ehmann/Selmayr (Hrsg.), DS-GVO).

Eichelberger, Jan/Wirth, Thomas/Seifert, Fedor, Urheberrechtsgesetz mit Verwertungsgesellschaftengesetz. Handkommentar, 3. Auflage, Baden-Baden 2020 (zitiert als Bearbeiter in: HK-UrhG).

Elzer, Oliver, Anmerkung zu BGH, Beschluss v. 27.08.2020 - III ZB 30/20, FD-ZVR 2020, 432500.

Emmerich, Volker/Lange, Knut Werner, Unlauterer Wettbewerb. Ein Studienbuch, 11. Auflage, München 2019 (zitiert als Emmerich/Lange, Unlauterer Wettbewerb).

Engeler, Malte, Der staatliche Twitter-Auftritt. Rechtliche Hürden und mögliche Lösungen, MMR 2017, S. 651-656.

Ensthaler, Jürgen, Industrie 4.0 und die Berechtigung an Daten, NJW 2016, S. 3473-3478.

ders./Weidert, Stefan, Handbuch Urheberrecht und Internet, 3. Auflage, Frankfurt am Main 2017 (zitiert als Bearbeiter in: Ensthaler/Weidert (Hrsg.), Urheberrecht und Internet).

Epping, Volker/Hillgruber, Christian, Beck'scher Online-Kommentar Grundgesetz, 44. Edition, München August 2020 (zitiert als Bearbeiter in: BeckOK Grundgesetz).

Ernst, Stefan, Die Einwilligung nach der Datenschutzgrundverordnung. Anmerkungen zur Definition nach Art. 4 Nr. 11 DS-GVO, ZD 2017, S. 110-114.

Eßer, Martin/Kramer, Philipp/Lewinski, Kai von, Auernhammer. Datenschutz-Grundverordnung, Bundesdatenschutzgesetz und Nebengesetze. Kommentar, 7. Auflage, Hürth 2020 (zitiert als Bearbeiter in: Auernhammer).

Faust, Florian, Digitale Wirtschaft - Analoges Recht: Braucht das BGB ein Update? Gutachten A zum 71. Deutschen Juristentag, München 2016 (zitiert als Faust, Gutachten zum 71. DJT, 2016).

Faust, Sebastian/Spittka, Jan/Wybitul, Tim, Milliardenbußgelder nach der DS-GVO? Ein Überblick über die neuen Sanktionen bei Verstößen gegen den Datenschutz, ZD 2016, S. 120-125.

Ferrari, Franco/Kieninger, Eva-Maria/Mankowski, Peter/Otte, Karsten/Saenger, Ingo u.a., Internationales Vertragsrecht. Rom I-VO. CISG. CMR. FactÜ. Kommentar, 3. Auflage, München 2018 (zit. als Bearbeiter in: Ferrari u.a. (Hrsg.), Internationales Vertragsrecht).

Fest, Timo, Anmerkung zu BGH, Urteil vom 08.10.2013 - XI ZR 401/12, JZ 2014, S. 152-156. 
Fezer, Karl-Heinz, Dateneigentum der Bürger. Ein originäres Immaterialgüterrecht sui generis an verhaltensgenerierten Informationsdaten der Bürger, ZD 2017, S. 99-105.

ders., Dateneigentum. Theorie des immaterialgüterrechtlichen Eigentums an verhaltensgenerierten Personendaten der Nutzer als Datenproduzenten, MMR 2017, S. 3-5.

Frank, Rainer/Helms, Tobias, Erbrecht, 7. Auflage, München 2018.

Fries, Peter, Influencer-Marketing. Informationspflichten bei Werbung durch Meinungsführer in Social Media, Wiesbaden 2019.

Fußeder, Florian, Soziale Netzwerke im Nachlass. Eine Untersuchung zum postmortalen Geheimnisschutz, Baden-Baden 2019 (zitiert als Fußeder, Soziale Netzwerke im Nachlass).

Funk, Stephanie, Das Erbe im Netz. Rechtslage und Praxis des digitalen Nachlasses, Wiesbaden 2017 (zitiert als Funk, Das Erbe im Netz).

Geppert, Martin/Schütz, Raimund, Beck'scher TKG-Kommentar, 4. Auflage, München 2013 (zitiert als Bearbeiter in: Beck'scher TKG-Kommentar).

Gergen, Thomas, Zur Schnittmenge von Erbrecht und Urheberrecht: die Nachfolge in die Rechte eines verstorbenen Urhebers, ZErb 2009, S. 42-48.

Gersdorf, Hubertus, Telekommunikationsrechtliche Einordnung von OTT-Diensten am Beispiel von Gmail. Rechtsgutachten im Auftrag der Google Inc., K\&R 2016, S. 91-101.

ders./Paal, Boris P., Beck'scher Online-Kommentar Informations- und Medienrecht, 29. Edition, München August 2020 (zitiert als Bearbeiter in: BeckOK Informations- und Medienrecht).

Gläser, Isabel, Anwendbares Recht auf Plattformverträge. Fragen des IPR bei sozialen Netzwerken am Beispiel von Facebook, MMR 2015, S. 699-704.

Gloser, Stefan, Die Rechtsnachfolge in das Urheberrecht, Köln 2012.

ders. Urheberrechte im Nachlass, DNotZ 2013, S. 497-522.

ders., „Digitale Erblasser“ und „digitale Vorsorgefälle“ - Herausforderungen der Online-Welt in der notariellen Praxis - Teil I, MittBayNot 2016, S. 12-19 und Teil II, MittBayNot 2016, S. 101-108.

ders. Anmerkung zu LG Berlin, Urteil v. 17.12.2015 - 20 O 172/15, DNotZ 2016, S. 545-549.

ders., Anmerkung zu KG Berlin, Urteil v. 31.05.2017 - 21 U 9/16, DNotZ 2018, S. 306-311.

ders., Anmerkung zu BGH, Urteil v. 12.07.2018 - III ZR 183/17, DNotZ 2018, S. 859-866.

Götting, Horst-Peter/Nordemann, Axel, UWG. Handkommentar, 3. Auflage, BadenBaden 2016 (zitiert als Bearbeiter in: HK-UWG).

Götting, Horst-Peter/Schertz, Christian/Seitz, Walter, Handbuch Persönlichkeitsrecht. Presse- und Medienrecht, 2. Auflage, München 2019 (zitiert als Bearbeiter in: Götting/Schertz/Seitz (Hrsg.), Handbuch Persönlichkeitsrecht). 
Gola, Peter, Datenschutz-Grundverordnung. Kommentar, 2. Auflage, München 2018 (zitiert als Bearbeiter in: Gola (Hrsg.), DS-GVO).

Gomille, Christian, Information als Nachlassgegenstand. Zugleich Besprechung von BGH, Urteil v. 12.07.2018 - III ZR 183/17, ZUM 2018, S. 660-667.

Goratsch, Patricia, Anmerkung zu BGH, Urteil v. 12.07.2018 - III ZR 183/17, NZFam 2018, S. 810-811.

Grabenwarter, Christoph, European Convention on Human Rights. Commentary, München 2014 (zitiert als Grabenwarter, European Convention on Human Rights).

ders./Pabel, Katharina, Europäische Menschenrechtskonvention. Ein Studienbuch, 6. Auflage, München 2016 (zitiert als Grabenwarter/Pabel, Europäische Menschenrechtskonvention).

Gräber, Tobias, Rechte an Accounts und virtuellen Gütern. Eine Untersuchung zu Fragen der Rechtsbeziehung zu Accounts und virtuellen Gütern in OnlineComputerspielen am Beispiel des Browserspiels OGame, Norderstedt 2009 (zitiert als Gräber, Rechte an Accounts).

Groll, Klaus Michael (Begr.)/Steiner, Anton, Praxishandbuch Erbrechtsberatung, 5. Auflage, Köln 2019 (zitiert als Bearbeiter in: Groll/Steiner (Hrsg.), Praxishandbuch Erbrechtsberatung).

Große-Wilde, Franz/Ouart, Peter, Deutscher Erbrechtskommentar, 2. Auflage, Köln 2010 (zitiert als Bearbeiter in: Große-Wilde/Ouart (Hrsg.), Deutscher Erbrechtskommentar).

Grünwald, Andreas/Nüßing, Christoph, Kommunikation over the Top. Regulierung für Skype, WhatsApp oder Gmail?, MMR 2016, S. 91-96.

Grützmacher, Malte, Wem gehören die Daten bei Industrie 4.0, Internet der Dinge und Connected Cars?, CR 2016, S. 485-495.

Gubitz, Daniel, Der Wettlauf zwischen Erben und Begünstigtem beim Vertrag zugunsten Dritter auf den Todesfall ist vermeidbar!, ZEV 2006, S. 333-338.

Haase, Martin Sebastian, Rechtsfragen des digitalen Nachlasses, in: Taeger, Jürgen (Hrsg.), Law as a Service - Recht im Internet- und Cloud-Zeitalter, Tagungsband DSRI-Herbstakademie 2013, Edewecht 2013, S. 379-393 (zitiert als Haase, Rechtsfragen des digitalen Nachlasses in: Taeger (Hrsg.), Tagungsband DSRIHerbstakademie 2013).

Hacker, Philipp, Daten als Gegenleistung: Rechtsgeschäfte im Spannungsfeld von DS-GVO und allgemeinem Vertragsrecht, ZfPW 2019, S. 148-197.

Härting, Niko, Internetrecht, 6. Auflage, Köln 2017

ders., „Dateneigentum“ - Schutz durch Immaterialgüterrecht? Was sich aus dem Verständnis von Software für den zivilrechtlichen Umgang mit Daten gewinnen lässt, CR 2016, S. 646-649.

ders./Dag, Seda, Vererblichkeit digitaler Benutzerkonten, K\&R 2018, S. 638-640. 
Hager, Günter, Neuere Tendenzen beim Vertrag zugunsten Dritter auf den Todesfall, in: Ficker, Hans Claudius/König, Detlef/Kreuzer, Karl u.a. (Hrsg.), Festschrift für Ernst von Caemmerer zum 70. Geburtstag, Tübingen 1978, S. 127-147 (zitiert als Hager in: FS von Caemmerer).

Haratsch, Andreas/Koenig, Christian/Pechstein, Matthias, Europarecht, 11. Auflage, Tübingen 2018.

Harte-Bavendamm/Ohly, Ansgar/Kalbfus, Björn, Gesetz zum Schutz von Geschäftsgeheimnissen. Kommentar, München 2020 (zitiert als Bearbeiter in: Harte-Bavendamm/Ohly/Kalbfus (Hrsg.), GeschGehG).

Hasselblatt, Gordian N., Münchener Anwaltshandbuch Gewerblicher Rechtsschutz, 5. Auflage, München 2017 (zitiert als Bearbeiter in: Münchener Anwaltshandbuch Gewerblicher Rechtsschutz).

Hau, Wolfgang/Poseck, Roman, Beck'scher Online Kommentar Bürgerliches Gesetzbuch, 55. Edition, München August 2020 (zitiert als Bearbeiter in: BeckOK BGB).

Heintz, Veris-Pascal/Ludyga, Hannes, Endlich Rechtsklarheit beim digitalen Nachlass? Das „Facebook-Urteil“ des BGH, jM 2018, S. 398-403.

Herfurth, Constantin, Interessenabwägung nach Art. 6 Abs. 1 lit.f DS-GVO. Nachvollziehbare Ergebnisse anhand von 15 Kriterien mit dem sog. „3x5-Modell“, ZD 2018, S. 514-520.

Hermes, Kai, Games: Aktuelle Rechtsfragen zum Handel mit virtuellen Gegenständen, GRUR-Prax 2013, S. 400-402.

Hertin, Paul/Wagner, Sandra, Urheberrecht, 3. Auflage, München 2019.

Herzog, Stephanie, Der digitale Nachlass - ein bisher kaum gesehenes und häufig missverstandenes Problem, NJW 2013, S. 3745-3751.

dies., Facebook und der digitale Nachlass. Anmerkung zu KG Berlin, Urteil v. 31.05.2017 - 21 U 9/16, ZErb 2017, S. 205-210.

dies., Der digitale Nachlass und das Erbrecht. Universalsukzession gilt auch in der digitalen Welt - vorsorgende Rechtspflege stärken, AnwBl Online 2018, S. 472-481.

dies./Pruns, Matthias, Der digitale Nachlass in der Vorsorge- und Erbrechtspraxis, Bonn 2018.

Hetmank, Sven, Internetrecht. Grundlagen - Streitfragen - Aktuelle Entwicklungen, Wiesbaden 2016 (zitiert als Hetmank, Internetrecht).

Hoeren, Thomas, Der Tod und das Internet - Rechtliche Fragen zur Verwendung von E-Mail- und WWW-Accounts nach dem Tode des Inhabers, NJW 2005, S. 2113-2117.

ders., Internetrecht, 3. Auflage, Berlin/Boston 2018.

ders., Anmerkung zu BGH, Urteil v. 12.7.2018 - III ZR 183/17, MMR 2018, S. 749-750.

ders., Datenbesitz statt Dateneigentum. Erste Ansätze zur Neuausrichtung der Diskussion um die Zuordnung von Daten MMR 2019, S. 5-8. 
ders., Anmerkung zu KG Berlin, Beschluss v. 9.12.2019 - 21 W 11/19, MMR 2020, S. 183-184.

ders./Pinelli, Stefan, Daten im Rechtsverkehr - Überlegungen für ein allgemeines Datenvertragsrecht, JZ 2020, S. 879-884.

ders./Sieber, Ulrich/Holznagel, Bernd, Handbuch Multimedia-Recht. Rechtsfragen des elektronischen Geschäftsverkehrs, 53. Ergänzungslieferung, München August 2020 (zitiert als Bearbeiter in: Hoeren/Sieber/Holznagel (Hrsg.), MMR$\mathrm{HdB})$.

Hoblfeld, Ralf/Godulla, Alexander, Das Phänomen der Sozialen Medien, in: Hornung, Gerrit/Müller-Terpitz, Ralf (Hrsg.), Rechtshandbuch Social Media, Berlin, Heidelberg 2015.

Hüßtege, Rainer/Mansel, Heinz-Peter, Nomos Kommentar. Rom-Verordnungen, 3. Auflage, Baden-Baden 2019 (zitiert als Bearbeiter in: NK-Rom VO).

Ipsen, Jörn, Staatsrecht II. Grundrechte, 23. Auflage, München 2020.

Isensee, Josef/Kirchhof, Paul, Handbuch des Staatsrechts der Bundesrepublik Deutschland. Band VIII, Grundrechte: Wirtschaft, Verfahren, Gleichheit, 3. Auflage, Heidelberg, München, Landsberg u.a. 2010 (zitiert als Bearbeiter in: Isensee/Kirchhof (Hrsg.), Handbuch des Staatsrechts).

Janal, Ruth, Die AGB-Einbeziehung im „M-Commerce“, NJW 2016, S. 3201-3205.

Jandt, Silke/Roßnagel, Alexander, Social Networks für Kinder und Jugendliche. Besteht ein ausreichender Datenschutz?, MMR 2011, S. 637-642.

Jarass, Hans/Pieroth, Bodo, Grundgesetz für die Bundesrepublik Deutschland. Kommentar, 16. Auflage, München 2020 (zitiert als Bearbeiter in: Jarass/Pieroth (Hrsg.), GG).

Jarass, Hans/Kment, Martin, EU-Grundrechte, 2. Auflage, München 2019.

Jauernig, Othmar (Begr.)/Stürner, Rolf (Hrsg.), Bürgerliches Gesetzbuch mit RomI-, Rom-II-VO, EuUnthVO/HUntProt und EuErbVO. Kommentar, 17. Auflage, München 2018 (zitiert als Bearbeiter in: Jauernig, BGB).

Junker, Abbo, Internationales Privatrecht, 3. Auflage, München 2019.

Kahl, Wolfgang/Waldhoff, Christian/Walter, Christian, Bonner Kommentar zum Grundgesetz, 207. Aktualisierung, Heidelberg September 2020 (zitiert als Bearbeiter in: Bonner Kommentar, GG).

Kannowski, Bernd/Till, Jan-Niklas, Der In-App-Kauf als neuer Vertragstyp, NJOZ 2017, S. 552-526.

Keim, Christopher, Der Erbnachweis gegenüber Banken nach dem BGH-Urteil vom 8.10.2013, ZEV 2014, S. 277-282.

Kerber, Wolfgang, A New (Intellectual) Property Right for Non-Personal Data? An Economic Analysis, GRUR Int. 2016, S. 989-998. 
Kindl, Johann, Verträge über digitale Inhalte - Vertragsnatur und geschuldete Leistung, in: Kindl, Johann/Arroyo Vendrell, Tatiana/Gsell, Beate (Hrsg.), Verträge über digitale Inhalte und digitale Dienstleistungen, Baden-Baden 2018, S. 63-83.

Kiparski, Gerd/Sassenberg, Thomas, DSGVO und TK-Datenschutz - Ein komplexes europarechtliches Geflecht. Welche bereichsspezifischen Datenschutzregelungen im TKG werden durch die DSGVO verdrängt?, CR 2018, S. 324-330.

Klages, Christlieb, Anmerkung zu KG Berlin, Urteil v. 31.05.2017 - 21 U 9/16, ZD 2017, S. 390-391.

Klas, Benedikt/Möhrke-Sobolewski, Christine, Digitaler Nachlass - Erbenschutz trotz Datenschutz, NJW 2015, S. 3473-3478.

Kleespies, Mathias, Die Domain als selbstständiger Vermögensgegenstand in der Einzelzwangsvollstreckung, GRUR 2002, S. 764-775.

Knoop, Martina, Digitaler Nachlass - Vererbbarkeit von Konten (minderjähriger) Nutzer in Sozialen Netzwerken, NZFam 2016, S. 966-970.

Köhler, Helmut/Bornkamm, Joachim/Feddersen, Jörn, Gesetz gegen den unlauteren Wettbewerb. GeschGehG. PAngV. UKlaG. DL-InfoV, 38. Auflage, München 2020 (zitiert als Bearbeiter in: Köhler/Bornkamm/Feddersen (Hrsg.), UWG).

Köhler, Markus, \#Alles Werbung? - Sind Influencer-Posts redaktionell getarnte Werbung? - Anmerkung zu KG Berlin, Urteil v. 8.1.2019 - 5 U 83/18, ZUMRD 2019, S. 141-144.

Kosmides, Timoleon, Providing-Verträge. Systematik und Methodologie der Bestimmung von Rechtsnatur und Rechtsfolgen, München 2010 (zitiert als Kosmides, Providing-Verträge).

Krieg, Henning, Twitter und Recht. Kurze Tweets, große Wirkung - die rechtlichen Stolperfallen beim Twittern, K\&R 2010, S. 73-77.

Kroiß, Ludwig, Anmerkung zu BGH, Urteil v. 5.4.2016 - XI ZR 440/15, NJW 2016, S. 2411-2412.

ders./Horn, Claus-Henrik/Solomon, Dennis, Nomos Kommentar. Nachfolgerecht. Erbrechtliche Spezialgesetze. Zivilrecht. Strafrecht. Verwaltungsrecht. Steuerrecht. Verfahrensrecht. Internationales Erbrecht, 2. Auflage, Baden-Baden 2019 (zitiert als Bearbeiter in: NK-Nachfolgerecht).

Kuntz, Wolfgang, Digitaler Nachlass: Zugang der Erben zum Facebook-Nutzerkonto, Anmerkung zu LG Berlin, Urteil v. 17.12.2015 - 20 O 172/151, jM 2016, S. 190-192.

Kutscher, Antonia, Der digitale Nachlass, Göttingen 2015.

Kühling, Jürgen, Neues Bundesdatenschutzgesetz - Anpassungsbedarf bei Unternehmen, NJW 2017, S. 1985-1990.

ders./Buchner, Benedikt, Datenschutz-Grundverordnung/BDSG. Kommentar, 3. Auflage, München 2020 (zitiert als Bearbeiter in: Kühling/Buchner (Hrsg.), DSGVO).

ders./Klar, Manuel/Sackmann, Florian, Datenschutzrecht, 4. Auflage, Heidelberg 2018. 
ders./Martini, Mario u.a., Die Datenschutz-Grundverordnung und das nationale Recht. Erste Überlegungen zum innerstaatlichen Regelungsbedarf, Münster 2016 (zitiert als Kühling/Martini u.a., Die DSGVO und das nationale Recht).

ders./Sackmann, Florian, Irrweg „Dateneigentum“. Neue Großkonzepte als Hemmnis für die Nutzung und Kommerzialisierung von Daten, ZD 2020, S. 24-30.

ders./Schall, Tobias, WhatsApp, Skype \& Co. - OTT-Kommunikationsdienste im Spiegel des geltenden Telekommunikationsrechts. „Level playing field“ de lege lata oder de lege ferenda?, CR 2015, S. 641-655

dies., E-Mail-Dienste sind Telekommunikationsdienste i.S.d. $\$ 3$ Nr. 24 TKG, CR 2016, S. 185-198.

dies./Biendl, Michael, Telekommunikationsrecht, 2. Auflage, Heidelberg, München, Landsberg u.a. 2014.

Lange, Heinrich (Begr.)/Kuchinke, Kurt, Erbrecht. Ein Lehrbuch, 5. Auflage, München 2001.

Lange, Knut Werner, Erbrecht, 2. Auflage, München 2017.

ders./Holtwiesche, Marian, Digitaler Nachlass - eine Herausforderung für Wissenschaft und Praxis (Teil 1), ZErb 2016, S. 125-131 und (Teil 2), ZErb 2016, S. 157-162.

Leeb, Christina-Maria, Bekannt verstorben - Rechtsfragen des Umgangs mit Social Media Daten Verstorbener, K\&R 2014, S. 693-703.

dies./Maisch, Marc, Social-Media-Stars und -Sternchen im rechtsfreien Raum? Aspekte eines "Influencer-Rechts«, ZUM 2019, S. 29-40.

Lettl, Tobias, Gewerblicher Rechtsschutz, München 2019

Leipold, Dieter, Erbrecht. Ein Lehrbuch mit Fällen und Kontrollfragen, 22. Auflage, Tübingen 2020 (zitiert als Leipold, Erbrecht).

Lieder, Jan/Berneith, Daniel, Anmerkung zu LG Berlin, Urteil v. 17.12.2015 - $20 \mathrm{O}$ 172/15, FamRZ 2016, S. 743-744.

dies., Digitaler Nachlass: Das Facebook-Urteil des BGH, FamRZ 2018, S. 1486-1488. dies., Digitaler Nachlass - Sollte der Gesetzgeber tätig werden?, ZRP 2020, S. 87-89. dies., Anmerkung zu BGH, Beschluss v. 27.8.2029 - III ZB 30/20, FamRZ 2020, S. 1761-1762.

Linardatos, Dimitrios/Schwarz, Philip, Anmerkung zu BGH, Urteil vom 08.10.2013 XI ZR 401/12, EWiR 2013, S. 731-732.

Litzenburger, Wolfgang, Anmerkung zu LG Berlin, Urteil v. 17.12.2015 - $20 \mathrm{O}$ 172/15, FD-ErbR 2016, 375286.

ders., Anmerkung zu BGH, Urteil v. 12.07.2018 - III ZR 183/17, FD-ErbR 2018, 407688.

Loewenheim, Ulrich, Handbuch des Urheberrechts, 2. Auflage, München 2010 (zitiert als Bearbeiter in: Loewenheim (Hrsg.), Handbuch des Urheberrechts).

Looschelders, Dirk, Schuldrecht. Allgemeiner Teil, 18. Auflage, München 2020. 
Ludwigs, Markus/Huller, Felix, OTT-Kommunikation: (Noch) Keine TK-Regulierung für Gmail \& Co., NVwZ 2019, S. 1099-1101.

Ludyga, Hannes, Der digitale Nachlass - zivilrechtliche Aspekte, jM 2016, S. 442-447.

ders., „Digitales Update“ für das Erbrecht im BGB?, ZEV 2018, S. 1-6.

ders., Anmerkung zu BGH, Urteil v. 12.7.2018 - III ZR 183/17, ZEV 2018, S. 592-593.

Lübbe-Wolff, Gertrude, Das Prinzip der praktischen Konkordanz, in: Herrmann, Dirk/Krämer, Achim (Hrsg.), Festschrift für Christian Kirchberg zum 70. Geburtstag am 5. September 2017, Stuttgart, München, Hannover u.a. 2017, S. 143-154 (zitiert als Lübbe-Wolff, FS Kirchberg).

Mangoldt, Hermann v. (Begr.)/Klein, Friedrich/Starck, Christian/Huber, Peter M./ Voßkuble, Andreas (Hrsg.), Grundgesetz, Kommentar, Band 1, Präambel, Artikel 1-19, 7. Auflage, München 2018 (zitiert als Bearbeiter in: v. Mangoldt/Klein/ Starck, GG).

Markendorf, Merih, Recht an Daten in der deutschen Rechtsordnung. Blockchain als Lösungsansatz für eine rechtliche Zuordnung?, ZD 2018, S. 409-413.

Marsch, Nikolaus, Das europäische Datenschutzgrundrecht. Grundlagen - Dimensionen - Verflechtungen, Tübingen 2018 (zitiert als Marsch, Das europäische Datenschutzgrundrecht).

Martini, Mario, Der digitale Nachlass und die Herausforderung postmortalen Persönlichkeitsschutzes im Internet, JZ 2012, S. 1145-1155.

ders./Kienle, Thomas, Facebook, die Lebenden und die Toten. Der digitale Nachlass aus telekommunikations- und datenschutzrechtlicher Sicht - zugleich Besprechung von BGH, Urteil v. 12.7.2018 - III ZR 183/17, JZ 2019, S. 235-241.

Maunz, Theodor/Dürig, Günter (Begr.)/Herzog, Roman/Scholz, Rupert/Herdegen, Matthias/Klein, Hans H. (Hrsg.), Grundgesetz, Kommentar, 91. Ergänzungslieferung, München April 2020 (zitiert als Bearbeiter in: Maunz/Dürig (Begr.), GG).

Mayen, Thomas, Das Fernmeldegeheimnis und der digitale Nachlass. Warum das Erbrecht nicht ausreicht, um den digitalen Nachlass rechtlich zu bewältigen, AnwBl Online 2018, S. 466-471.

ders., Über die mittelbare Grundrechtsbindung Privater in Zeiten des Einflusses sozialer Netzwerke auf die öffentliche Kommunikation, ZHR 182 (2018), S. 1-7.

Mayer-Schönberger, Viktor, Delete. Die Tugend des Vergessens in digitalen Zeiten, Berlin 2010 (zitiert als Mayer-Schönberger, Delete).

Metzger, Axel, Dienst gegen Daten: Ein synallagmatischer Vertrag, AcP 216 (2016), S. 817-865.

Meyer-Ladewig, Jens/Nettesheim, Martin/Raumer, Stefan v., EMRK. Europäische Menschenrechtskonvention. Handkommentar, 4. Auflage, Baden-Baden 2017 (zitiert als Bearbeiter in: HK-EMRK).

Mischau, Lena, Daten als "Gegenleistung“ im neuen Verbrauchervertragsrecht, ZEuP 2020, S. 335-365. 
Müller-Broich, Jan, Nomos Kommentar. Telemediengesetz, Baden-Baden 2012 (zitiert als Bearbeiter in: NK-TMG).

Muscheler, Karlheinz, Vertrag zugunsten Dritter auf den Todesfall und Erbenwiderruf, WM 1994, S. 921-938.

ders., Erbrecht. Band I, Tübingen 2010.

Nordemann, Axel/Nordemann, Jan Bernd/Czychowski, Christian, Fromm/Nordemann. Urheberrecht. Kommentar zum Urheberrechtsgesetz, Verlagsgesetz, Einigungsvertrag (Urheberrecht), EU PortabilitätsVO, 12. Auflage, Stuttgart 2018 (zitiert als Bearbeiter in: Fromm/Nordemann).

Nordemann, Jan Bernd, AGB-Kontrolle von Nutzungsrechtseinräumungen durch den Urheber, NJW 2012, S. 3121-3125.

Nordmeier, Carl Friedrich, Cloud Computing und Internationales Privatrecht. Anwendbares Recht bei der Schädigung von in Datenwolken gespeicherten Daten, MMR 2010, S. 151-156.

Ohly, Ansgar, Gibt es einen Numerus clausus der Immaterialgüterrechte?, in: Ohly, Ansgar/Bodewig, Theo u.a. (Hrsg.), Perspektiven des Geistigen Eigentums und Wettbewerbsrechts. Festschrift für Gerhard Schricker zum 70. Geburtstag, München 2005 (zitiert als Obly in: FS Schricker).

ders., Das neue Geschäftsgeheimnisgesetz im Überblick, GRUR 2019, 441-451.

Oppermann, Thomas/Classen, Claus Dieter/Nettesheim, Martin, Europarecht. Ein Studienbuch, 8. Auflage, München 2018.

Paal, Boris P., Immaterialgüter, Internetmonopole und Kartellrecht, GRUR 2013, S. 873-881.

ders., GWB-Update für die Digitalisierung: Zur Erweiterung des Missbrauchstatbestands, WuW 2016, S. 453.

ders., Schadensersatzansprüche bei Datenschutzverstößen. Voraussetzungen und Probleme des Art. 82 DS-GVO, MMR 2020, S. 14-19.

ders., Kritische Würdigung des Konzepts der Datenschutzaufsichtsbehörden zur Bußgeldzumessung, RDV 2020, S. 57-64.

ders./Hennemann, Moritz, Big Data im Recht. Wettbewerbs- und daten(schutz)rechtliche Herausforderungen, NJW 2017, S. 1697-1701.

ders../Pauly, Daniel A., Datenschutz-Grundverordnung/Bundesdatenschutzgesetz, 2. Auflage, München 2018 (zitiert als Bearbeiter, in: Paal/Pauly (Hrsg.), DS-GVO/ BDSG).

Palandt, Otto (Begr.), Palandt. Bürgerliches Gesetzbuch mit Nebengesetzen, 79. Auflage, München 2020 (zitiert als Bearbeiter in: Palandt).

Peifer, Karl-Nikolaus, Die AGB-Kontrolle von Urheberverträgen - Götterdämmerung für das Leitbild der Durchsetzung einer angemessenen Vergütung?, AfP 2012, S. 510-516. 
Pierson, Matthias/Abrens, Thomas/Fischer, Karsten R., Recht des geistigen Eigentums. Gewerblicher Rechtsschutz, Urheberrecht, Wettbewerbsrecht, 4. Auflage, Tübingen 2018 (zitiert als Pierson/Ahrens/Fischer, Recht des geistigen Eigentums).

Plath, Kai-Uwe, DSGVO/BDSG. Kommentar zu DSGVO, BDSG und den Datenschutzbestimmungen von TMG und TKG, 3. Auflage, Köln 2018 (zitiert als Bearbeiter in: Plath (Hrsg.), DS-GVO).

Podszun, Rupprecht, Wandlungen des Schutzgegenstands, in: Dreier, Thomas/Hilty, Reto (Hrsg.), Vom Magnettonband zu Social Media. Festschrift 50 Jahre Urhebergesetz, München 2015, S. 361-378 (zitiert als Podszun in: FS 50 Jahre UrhG).

ders., Anmerkung zu LG Berlin, Urteil v. 17.12.2015 - 20 O 172/15, GWR 2016, S. 37.

Preuß, Nicola, Digitaler Nachlass - Vererbbarkeit eines Kontos bei einem sozialen Netzwerk, NJW 2018, S. 3146-3149.

Prütting, Hanns/Wegen, Gerhard/Weinrich, Gerd, Bürgerliches Gesetzbuch. Kommentar, 14. Auflage, Köln 2019 (zitiert als Bearbeiter in: Prütting/Wegen/Weinrich (Hrsg.), BGB).

Pruns, Matthias, Keine Angst vor dem digitalen Nachlass! Erbrecht vs. Fernmeldegeheimnis?, NWB 2014, S. 2175-2186.

ders., Digitaler Nachlass - Irrungen und Wirrungen um $\$ 88$ Abs. 3 TKG, ZErb 2017, S. 217-224.

ders., Der digitale Nachlass in der Beratungspraxis nach dem Facebook-Urteil des BGH. Teil 1: Die Grundlagen der Rechtsnachfolge in das digitale Vermögen, ErbR 2018, S. 550-556

ders., Der digitale Nachlass in der Beratungspraxis nach dem Facebook-Urteil des BGH. Teil 2: Soziale Netzwerke - Rechtliche Durchsetzung - Vorsorge, ErbR 2018, S. 614-622.

ders., Anmerkung zu LG Münster, Versäumnisurteil v. 16.4.2019 - 14 O 565/18, in: ErbR 2019, S. 455-457.

Radlanski, Philip, Das Konzept der Einwilligung in der datenschutzrechtlichen Realität, Tübingen 2016.

Ragno, Francesca, The Law Applicable to Consumer Contracts under the Rome I Regulation, in: Ferrari, Franco/Leible, Stefan (Hrsg.), Rome I Regulation. The Law Applicable to Contractual Obligations in Europe, München 2009 (zitiert als Ragno in: Leible/Ferrari (Hrsg.), Rome I Regulation).

Rauda, Christian, Der Rückruf wegen gewandelter Überzeugung nach $\$ 42$ UrhG. Von Web 2.0 aus dem Dornröschenschlaf geweckt?, GRUR 2010, S. 22-27.

Raude, Karin, Der digitale Nachlass in der notariellen Praxis, RNotZ 2017, S. 17-27.

dies., Rechtsprobleme des digitalen Nachlasses: Der Anspruch der Erben auf Zugang zum Account des Erblassers in sozialen Netzwerken, ZEV 2017, S. 433-439.

Raue, Peter/Hegemann, Jan, Münchener Anwaltshandbuch Urheber- und Medienrecht, 2. Auflage, München 2017 (zitiert als Bearbeiter in: Münchener Anwaltshandbuch Urheberrecht). 
Rauscher, Thomas, Internationales Privatrecht. Mit internationalem Verfahrensrecht, 5. Auflage, Heidelberg 2017 (zitiert als Rauscher, Internationales Privatrecht).

Redeker, Helmut, IT-Recht, 7. Auflage, München 2020.

ders., Handbuch der IT-Verträge, Lieferung 40, Köln April 2020 (zitiert als Bearbeiter in: Redeker (Hrsg.), IT-Verträge).

Rehbinder, Manfred/Peukert, Alexander, Urheberrecht und verwandte Schutzrechte, 18. Auflage, München 2018 (zitiert als Rebbinder/Peukert, Urheberrecht).

Reinemann, Susanne/Remmertz, Frank, Urheberrechte an User-generated Content, ZUM 2012, S. 216-227.

Richter, Frederick, Vorwort, in: Stiftung Datenschutz (Hrsg.), Dateneigentum und Datenhandel, Berlin 2019.

Rockstroh, Sebastian, Impressumspflicht auf Facebook-Seiten. Wann werden Telemedien „in der Regel gegen Entgelt” angeboten?, MMR 2013, S. 627-630.

Röhricht, Volker/Westphalen, Friedrich Graf v./Haas, Ulrich, Handelsgesetzbuch. Kommentar, 5. Auflage, Köln 2019 (zitiert als Bearbeiter in: Röhricht/Graf von Westphalen/Haas (Hrsg.), HGB).

Rössel, Markus, Vererblichkeit eines Facebook-Kontos. Anmerkung zu BGH, Urteil v. 12.07.2018 - III ZR 183/17, ITRB 2018, S. 224-225.

Röttgen, Charlotte, Rechtspositionen an Daten: Die Rechtslage im europäischen Rechtsraum, in: Specht-Riemenschneider, Louisa/Werry, Nikola/Werry, Susanne (Hrsg.), Datenrecht in der Digitalisierung, Berlin 2020, S. 371-407 (zitiert als Röttgen in: Specht-Riemenschneider/Werry/Werry (Hrsg.), Datenrecht in der Digitalisierung).

Röthel, Anne, Solidaritätskonzept und Statusorientierung des Erbrechts, in: Lipp, Volker/Röthel, Anne/Windel, Peter (Hrsg.), Familienrechtlicher Status und Solidarität, Tübingen 2008.

dies., Erbrecht. Ein Studienbuch, 18. Auflage, München 2020 (zitiert als Röthel, Erbrecht).

Roßnagel, Alexander, Der Datenschutz von Kindern in der DS-GVO. Vorschläge für die Evaluierung und Fortentwicklung, ZD 2020, S. 88-92.

Rott, Eberhard/Rott, Alexander, Wem gehört die E-Mail? Rechts- und Praxisprobleme beim digitalen Nachlass, NWB-EV 2013, S. 160-168. 
Säcker, Franz Jürgen/Rixecker, Roland/Oetker, Hartmut/Limperg, Bettina, Münchener Kommentar zum Bürgerlichen Gesetzbuch. Band 1. Allgemeiner Teil, 8. Auflage, München 2018. Band 2. Schuldrecht - Allgemeiner Teil I, 8. Auflage, München 2019. Band 3. Schuldrecht - Allgemeiner Teil II, 8. Auflage, München 2019. Band 5. Schuldrecht - Besonderer Teil II, 8. Auflage, München 2020. Band 6. Schuldrecht - Besonderer Teil III, 8. Auflage, München 2020. Band 7. Schuldrecht. Besonderer Teil IV, 8. Auflage, München 2020. Band 8. Sachenrecht, 8. Auflage, München 2020. Band 11. Erbrecht, 8. Auflage, München 2020. Band 12. Internationales Privatrecht I, 8. Auflage, München 2020. Band 12. Internationales Privatrecht II, 7. Auflage, München 2018 (zitiert als Bearbeiter in: MüKo BGB).

Salomon, Pascal, "Digitaler Nachlass" - Möglichkeiten der notariellen Vorsorge, NotBZ 2016, S. 324-331.

Schack, Haimo, Urheber- und Urhebervertragsrecht, 9. Auflage, Tübingen 2019.

Schantz, Peter, Die Datenschutz-Grundverordnung - Beginn einer neuen Zeitrechnung im Datenschutzrecht, NJW 2016, S. 1841-1847.

ders./Wolff, Heinrich Amadeus, Das neue Datenschutzrecht. Datenschutz-Grundverordnung und Bundesdatenschutzgesetz in der Praxis, München 2017 (zitiert als Schantz/Wolff, Das neue Datenschutzrecht).

Scherer, Stephan, Münchener Anwaltshandbuch Erbrecht, 5. Auflage, München 2018 (zitiert als Bearbeiter in: Münchener Anwaltshandbuch Erbrecht).

Scheurle, Klaus-Dieter/Mayen, Thomas, Telekommunikationsgesetz. Kommentar, 3. Auflage, München 2018 (zitiert als Bearbeiter in: Scheurle/Mayen (Hrsg.), TKG).

Schippan, Martin, Klare Worte des BGH zur Wirksamkeit von Honorarbedingungen für freie Journalisten. Zugleich Besprechung von BGH ZUM 2012, 793 Honorarbedingungen Freie Journalisten, ZUM 2012, S. 771-781.

Schmidt, Karsten, Münchener Kommentar zum Handelsgesetzbuch, Band 6. Bankvertragsrecht, 4. Auflage, München 2019 (zitiert als Bearbeiter in: MüKo HGB).

Schneider, Jochen, Schließt Art. 9 DS-GVO die Zulässigkeit der Verarbeitung bei Big Data aus? Überlegungen, wie weit die Untersagung bei besonderen Datenkategorien reicht, ZD 2017, S. 303-308.

ders., Datenschutz nach der EU-Datenschutz-Grundverordnung, 2. Auflage, München 2019.

Schneider, Mathias, WhatsApp \& Co. - Dilemma um anwendbare Datenschutzregeln. Problemstellung und Regelungsbedarf bei Smartphone-Messengern, ZD 2014, S. 231-237.

Schricker, Gerhard (Begr.)/Loewenheim, Ulrich/Leistner, Matthias/Obly, Ansgar (Hrsg.), Urheberrecht. Kommentar, 6. Auflage, München 2020 (zitiert als Bearbeiter in: Schricker/Loewenheim). 
Schroeder, Moritz, Numerus clausus der Immaterialgüterrechte? - Zum normativen Gehalt eines sog. Prinzips, in: Specht, Louisa/Lauber-Rönsberg, Anne/Becker, Maximilian (Hrsg.), Medienrecht im Medienumbruch. Junge Wissenschaft zum Gewerblichen Rechtsschutz, Urheber- und Medienrecht, Baden-Baden 2017, S. 9-43.

Schulze, Gernot, Die Übertragungszwecklehre - Auslegungsregel und Inhaltsnorm? Zugleich Besprechung zu BGH, Urteil v. 31.5.2012 - I ZR 73/10 - Honorarbedingungen Freie Journalisten, GRUR 2012, S. 993-996.

Schulze, Reiner, Bürgerliches Gesetzbuch. Handkommentar, 10. Auflage, Baden-Baden 2019 (zitiert als Bearbeiter in: HK-BGB).

Schumacher, Pascal, Anmerkung zu EuGH, Urteil v. 13.6.2019 - C-193/18, K\&R 2019, S. 490-491.

Schuster, Fabian, E-Mail-Dienste als Telekommunikationsdienste? Warum OTTDienste keine TK-Dienste sein können - zugleich Erwiderung auf Kühling/ Schall, CR 2015, 641 und VG Köln zu „Google Mail“, CR 2016, S. 173-185.

Schwab, Dieter, Persönlichkeitsrecht und Erbe, in: Damrau, Jürgen/Muscheler, Karlheinz (Hrsg.), Erbrecht und Vermögensnachfolge. System. Struktur. Vertrag. Festschrift für Manfred Bengel und Wolfgang Reimann zum 70. Geburtstag, München 2012, S. 345-356 (zitiert als Schwab, in: FS für Bengel und Reimann).

Schweitzer, Heike, Digitale Plattformen als private Gesetzgeber: Ein Perspektivwechsel für die europäische „Plattform-Regulierung“, ZEuP 2019, S. 1-12.

Seidler, Katharina, Digitaler Nachlass. Das postmortale Schicksal elektronischer Kommunikation. Frankfurt am Main 2016 (zitiert als Seidler, Digitaler Nachlass).

dies., Der digitale Nachlass - ein Zwischenstand, NZFam 2020, S. 141-145.

Simitis, Spiros/Hornung, Gerrit/Spiecker gen. Döhmann, Indra, Nomos Kommentar. Datenschutzrecht. DSGVO mit BDSG, Baden-Baden 2019 (zitiert als Bearbeiter in: NK-Datenschutzrecht).

Sodan, Helge, Grundgesetz, 4. Auflage, München 2018 (zitiert als Bearbeiter in: Sodan (Hrsg.), GG).

Solmecke, Christian/Dam, Annika, Wirksamkeit der Nutzungsbedingungen sozialer Netzwerke. Rechtskonforme Lösung nach dem AGB- und dem Urheberrecht, MMR 2012, S. 71-74.

Solmecke, Christian/Köbrich, Thomas/Schmitt, Robin, Der digitale Nachlass - haben Erben einen Auskunftsanspruch? Überblick über den rechtssicheren Umgang mit den Daten von Verstorbenen, MMR 2015, S. 291-295.

Solmecke, Christian/Schmitt, Robin, Anmerkung zu LG Berlin, Urteil v. 17.12.201520 O 172/15, ZD 2016, S. 186-187.

Sorge, Christoph, Digitaler Nachlass als Knäuel von Rechtsverhältnissen. Justizministerkonferenz sieht kaum Handlungsbedarf für den Gesetzgeber, MMR 2018, S. 372-377.

Specht, Louisa, Ausschließlichkeitsrechte an Daten - Notwendigkeit, Schutzumfang, Alternativen. Eine Erläuterung des gegenwärtigen Meinungsstands und Gedanken für eine zukünftige Ausgestaltung, CR 2016, S. 288-269. 


\section{Literaturverzeichnis}

dies., Das Verhältnis möglicher Datenrechte zum Datenschutzrecht, GRUR Int. 2017, S. 1040-1047.

dies./Mantz, Reto, Handbuch. Europäisches und deutsches Datenschutzrecht. Bereichsspezifischer Datenschutz in Privatwirtschaft und öffentlichem Sektor, München 2019 (zitiert als Bearbeiter in: Specht/Mantz (Hrsg.), Handbuch Europäisches und deutsches Datenschutzrecht).

Spies, Axel, Anmerkung zu EuGH, Urteil v. 13.6.2019 - C-193/18, MMR 2019, S. 514-517.

Spindler, Gerald, Verträge über digitale Inhalte - Anwendungsbereich und Ansätze. Vorschlag der EU-Kommission zu einer Richtlinie über Verträge zur Bereitstellung digitaler Inhalte, MMR 2016, S. 147-153.

ders./Schmitz, Peter/Liesching, Marc, Telemediengesetz mit Netzwerkdurchsetzungsgesetz. Kommentar, 2. Auflage, München 2018 (zitiert als Bearbeiter in: Spindler/Schmitz (Hrsg.), TMG).

ders./Schuster, Fabian, Recht der elektronischen Medien. Kommentar, 4. Auflage, München 2019 (zitiert als Bearbeiter in: Spindler/Schuster (Hrsg.), Recht der elektronischen Medien).

Staudinger, Julius v. (Begr.), J. von Staudingers Kommentar zum Bürgerlichen Gesetzbuch mit Einführungsgesetz und Nebengesetzen. Eckpfeiler des Zivilrechts, Neubearbeitung 2020. Buch 1. Allgemeiner Teil. \$\$ 90-124; 130-133 (Sachen und Tiere, Geschäftsfähigkeit, Willenserklärung), Neubearbeitung 2017. Buch 2. Recht der Schuldverhältnisse. $\mathbb{S}$ 255-304 (Leistungsstörungsrecht 1), Neubearbeitung 2019. $\$ \$ 305-310$; UKlaG (AGB-Recht 1 und Unterlassungsklagengesetz), Neubearbeitung 2019. Anhang zu $\$ \mathbb{S} 305-310$ (AGB-Recht 2: Ausgewählte Verträge; AGB-Kontrolle im Arbeitsrecht; Internationaler Geschäftsverkehr), Neubearbeitung 2019. $\$ \$ 311,311 \mathrm{a}-\mathrm{c}$ (Vertragsschluss), Neubearbeitung 2018. \$\$397-432 (Erlass, Abtretung, Schuldübernahme, Mehrheit von Schuldnern und Gläubigern), Neubearbeitung 2017. $\$ 823$ A - D (Unerlaubte Handlung 1), Neubearbeitung 2017. Buch 5. Erbrecht. Einleitung zum Erbrecht; S\$ 19221966 (Erbfolge), Neubearbeitung 2017. Einführungsgesetz zum Bürgerlichen Gesetzbuch/IPR. Einleitung zum Internationalen Privatrecht, Neubearbeitung 2019. Internationales Wirtschaftsrecht (Kollisionsrecht des KartellR, LauterkeitsR, MarkenR, PatentR und UrheberR), Neubearbeitung 2019. Einleitung zur Rom I-VO; Art. 1-10 Rom I-VO (Internationales Vertragsrecht 1), Neubearbeitung 2016 (zitiert als Bearbeiter in: Staudinger).

Steiner, Anton/Holzer, Anna, Praktische Empfehlungen zum digitalen Nachlass, ZEV 2015, S. 262-266.

Steinrötter, Björn/Rahimi, Henna/Tran, Lan Mai, Informationspflichten datenschutzrechtlich Verantwortlicher, EWS 2019, S. 301-307.

Stieper, Malte, Verbraucherschutz im Urheberrecht, VuR 2014, S. 261-262.

ders., Digital ist besser - Die Bereitstellung digitaler Inhalte als eigenständiger Vertragstypus, in: Alexander, Christian u.a. (Hrsg.), Festschrift für Helmut Köhler zum 70. Geburtstag, München 2014, S. $729-743$ (zitiert als Stieper in: FS Köhler).

Stimmel, Ulrike, Die Beurteilung von Lizenzverträgen unter der Rom I-Verordnung, GRUR Int. 2010, S. 783-792. 
Sucker, Reinhard, Der digitale Werkgenuss um Urheberrecht, Tübingen 2014.

Sudhoff, Heinrich, Unternehmensnachfolge, 5. Auflage, München 2005 (zitiert als Bearbeiter in: Sudhoff (Hrsg.), Unternehmensnachfolge).

Sydow, Gernot, Europäische Datenschutzgrundverordnung. Handkommentar, 2. Auflage, Baden-Baden 2018 (zitiert als Bearbeiter in: HK-DS-GVO).

Taeger, Jürgen, Datenschutzrecht. Einführung, Frankfurt am Main 2014

ders./Gabel, Detlev, Kommentar. DSGVO - BDSG, 3. Auflage, Frankfurt am Main 2019 (zitiert als Bearbeiter in: Taeger/Gabel (Hrsg.), DS-GVO).

Tavanti, Pascal, Datenverarbeitung zu Werbezwecken nach der DatenschutzGrundverordnung (Teil 1), RDV 2016, S. 231-240 und (Teil 2), RDV 2016, S. 295-306.

Thiesen, Michael, Daten in der Erbmasse. Der digitale Nachlass zwischen Erbgang und Rechtsdurchsetzung, Frankfurt am Main 2017 (zitiert als Thiesen, Daten in der Erbmasse).

Traschler, Thomas, Der Wettlauf um den digitalen Nachlass aus rechtsvergleichender Perspektive. Zugleich Anmerkung zu BGH, Urteil v. 12.07.2018 - III ZR 183/17, ZEuP 2020, S. 168-184.

Troge, Thorsten, Herausforderung: Influencer-Marketing, GRUR-Prax 2018, S. 87-89.

Uecker, Philip, Die Einwilligung im Datenschutzrecht und ihre Alternativen. Mögliche Lösungen für Unternehmen und Vereine, ZD 2019, S. 248-251.

Ubrenbacher, Pia Elisa, Digitales Testament und digitaler Nachlass, Frankfurt am Main 2017.

Veil, Winfried, Einwilligung oder berechtigtes Interesse? - Datenverarbeitung zwischen Skylla und Charybdis, NJW 2018, S. 3337-3344.

Wandtke, Artur-Axel, Ökonomischer Wert von persönlichen Daten. Diskussion des „Warencharakters“ von Daten aus persönlichkeits- und urheberrechtlicher Sicht, MMR 2017, S. 6-12.

ders., Urheberrecht, 7. Auflage, Berlin/Boston 2019.

ders./Bullinger, Winfried, Praxiskommentar Urheberrecht. UrhG, VGG, InsO, UKlaG, KUG, EVtr, InfoSoc-RL, 5. Auflage, München 2019 (zitiert als Bearbeiter in: Wandtke/Bullinger (Hrsg.), UrhG).

Webleit, Jonas, WhatsApp - Rechtsnatur des Vertrags zwischen IM-Dienst und Nutzer. Technische Voraussetzungen und rechtliche Bewertung, MMR 2018, S. 279-282.

Wellenhofer, Marina, Erbrecht: Vererbbarkeit eines Facebook-Accounts, Anmerkung zu BGH, Urteil v. 12.7.2018 - III ZR 183/17, JuS 2018, S. 1101-1104.

Welser, Marcus v., Die Wahrnehmung urheberpersönlichkeitsrechtlicher Befugnisse durch Dritte, Berlin 2000. 


\section{Literaturverzeichnis}

Wendehorst, Christiane/Westphalen, Friedrich Graf v., Das Verhältnis zwischen Datenschutz-Grundverordnung und AGB-Recht, NJW 2016, S. 3745-3750.

Westermann, Harm Peter/Grunewald, Barbara/Maier-Reimer, Georg, Erman. Bürgerliches Gesetzbuch. Handkommentar, Band I und II, 16. Auflage, Köln 2020 (zitiert als Bearbeiter in: Erman).

Wiebe, Andreas, Schutz von Maschinendaten durch das sui-generis-Schutzrecht für Datenbanken, GRUR 2017, S. 338-345.

Wille, Stefan, Die neue Leitbilddiskussion im Urhebervertragsrecht. Zugleich Anmerkung zu LG München I ZUM 2010, 825, ZUM 2011, S. 206-211.

Willems, Constantin, Erben 2.0 - zur Beschränkbarkeit der Rechtsnachfolge in das „digitale Vermögen“, ZfPW 2016, S. 494-512.

ders., Influencer als Unternehmer. Überlegungen zu $\$ 2$ Abs. 1 Nr. 6 UWG und $\$ \$ 14,112$ BGB, MMR 2018, S. 707-711.

Wolff, Heinrich Amadeus/Brink, Stefan, Beck'scher Online-Kommentar Datenschutzrecht, 33. Edition, München August 2020 (zitiert als Bearbeiter in: BeckOK Datenschutzrecht).

Wüsthof, Lucas, Anmerkung zu KG Berlin, Urteil v. 31.05.2017 - 21 U 9/16, ErbR 2017, S. 508-510.

ders., Anmerkung zu BGH, Urteil v. 12.7.2018 - III ZR 183/17, ErbR 2018, S. 579-582.

Wunderlin, Philipp/Bielajew, Andrzej, Digitaler Nachlass - was geschieht mit unseren „IT-Daten“ nach dem Tod?, IPRB 2014, S. 223-226.

Zech, Herbert, Information als Schutzgegenstand, Tübingen 2012.

ders., Daten als Wirtschaftsgut - Überlegungen zu einem „Recht des Datenerzeugers", CR 2015, S. 137-146.

ders., „Industrie 4.0“ - Rechtsrahmen für eine Datenwirtschaft im digitalen Binnenmarkt, GRUR 2015, S. 1151-1160.

Zieger, Christoph/Smirra, Nikolas, Fallstricke bei Big Data-Anwendungen. Rechtliche Gesichtspunkte bei der Analyse fremder Datenbestände, MMR 2013, S. 418-421. 